Medicinal plants and pharmaceutical medicines use in pregnant and lactating women in Ethiopia

Seid Mussa Ahmed

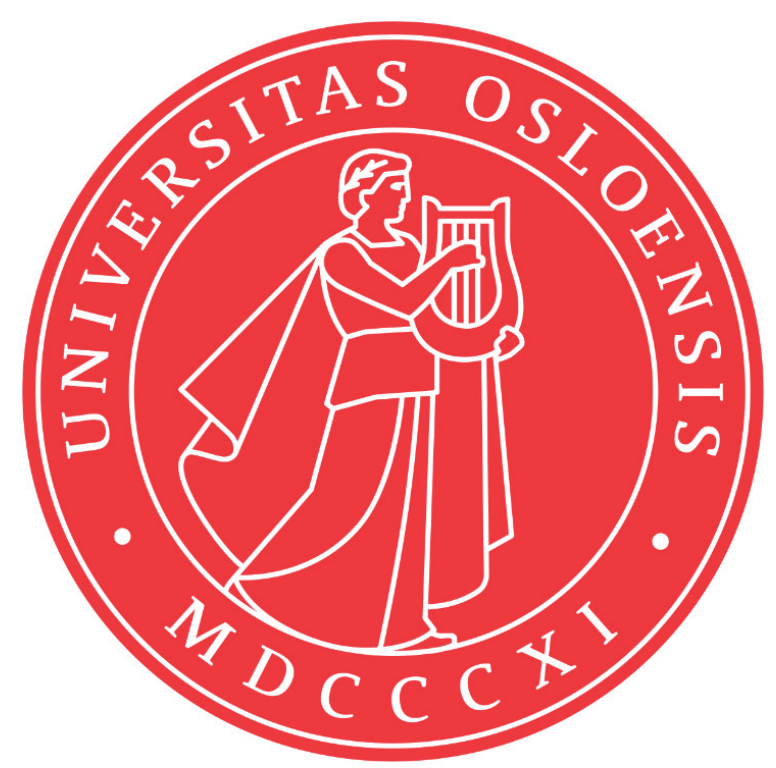

Dissertation for the degree of Philosophiae Doctor (PhD)

Department of Community Medicine and Global Health

Institute of Health and Society

Faculty of Medicine

University of Oslo

Oslo, Norway

2021 
(C) Seid Mussa Ahmed, 2021

Series of dissertations submitted to the Faculty of Medicine, University of Oslo

ISBN 978-82-8377-934-9

All rights reserved. No part of this publication may be reproduced or transmitted, in any form or by any means, without permission.

Cover: Hanne Baadsgaard Utigard.

Print production: Reprosentralen, University of Oslo. 


\section{Dedication}

To my daughters Aimal and Hamna. I love you to the moon and back. 

Table of Contents

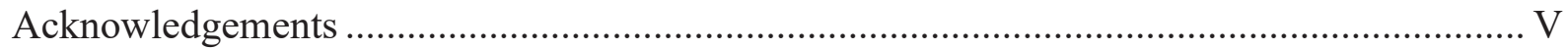

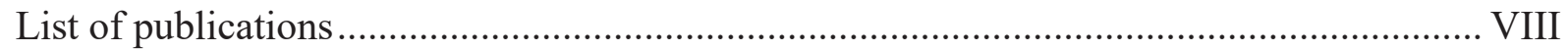

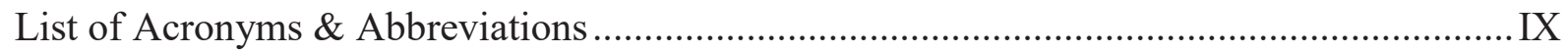

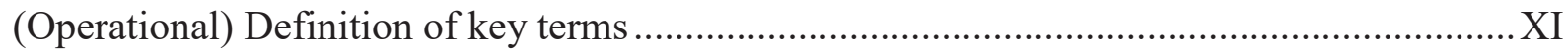

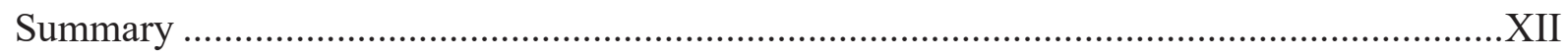

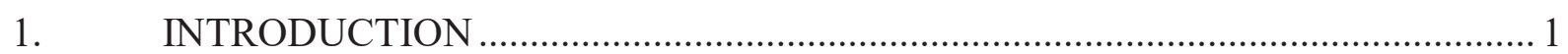

1.1. Medicinal plants and pharmaceutical medicines used in pregnancy ......................... 1

1.1.1. Medicinal plants used by pregnant women .................................................. 1

1.1.2. Pharmaceutical medicines used by pregnant women ....................................... 2

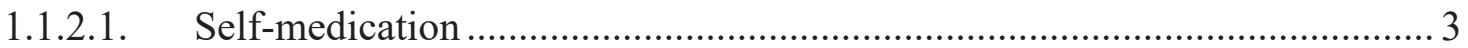

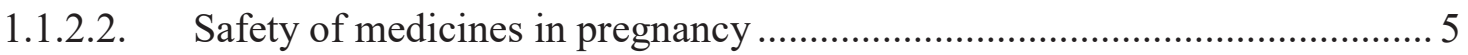

1.1.2.3. Pregnancy risk classification for medicines .......................................... 6

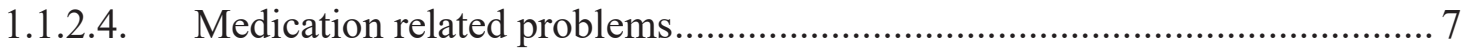

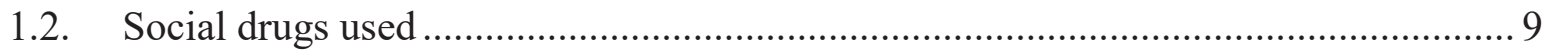

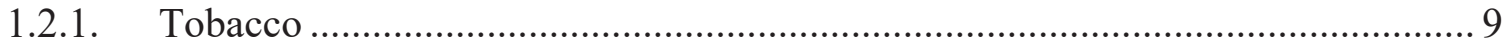

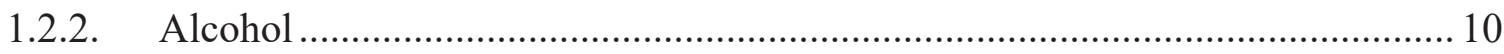

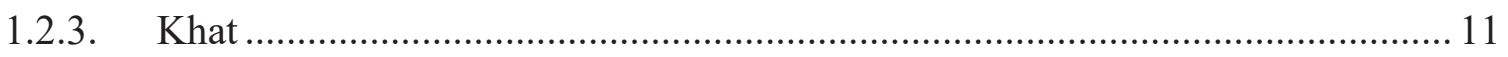

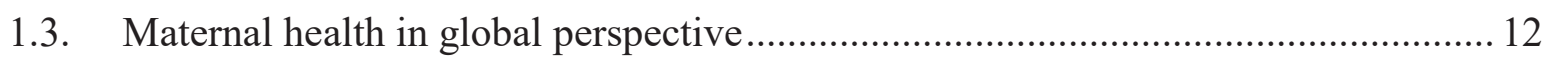

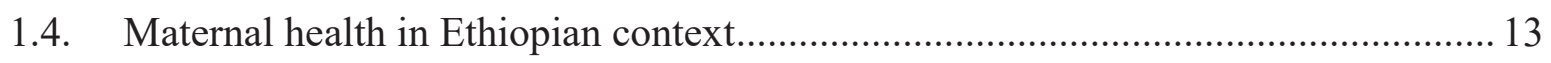

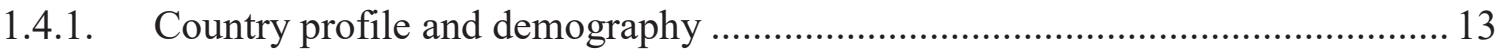

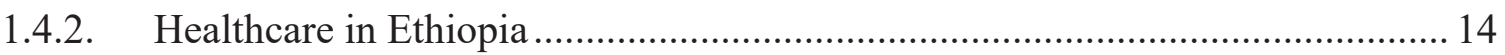

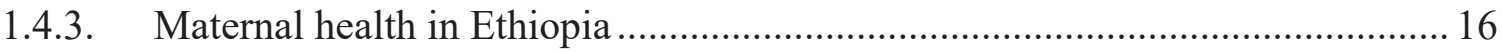

1.4.4. Millennium development goals and sustained development goals ................... 17

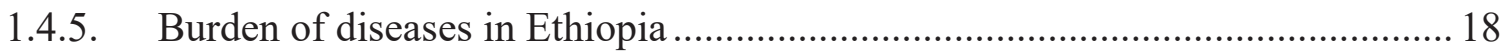

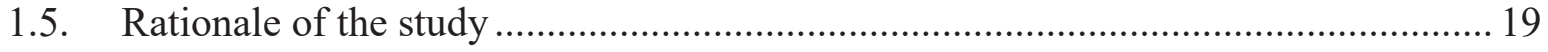

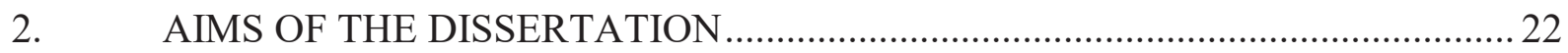




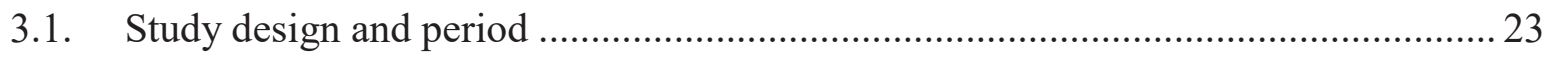

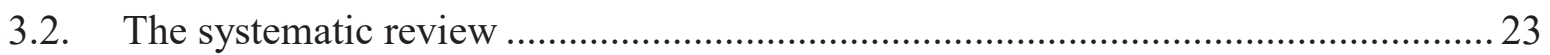

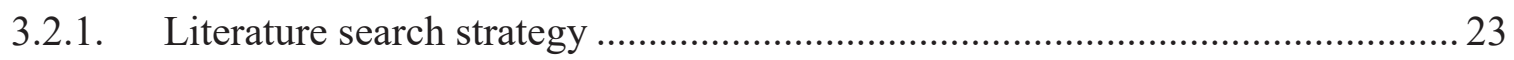

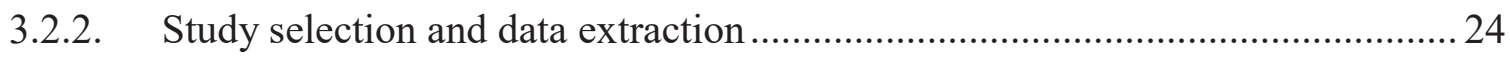

3.2.3. Studies included in the systematic review.................................................... 24

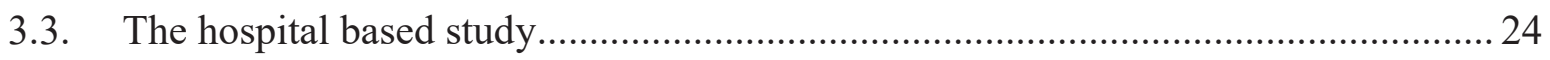

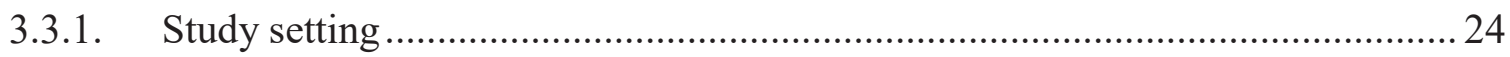

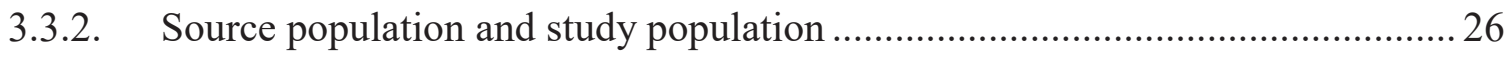

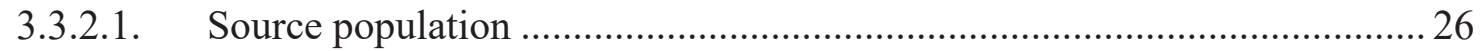

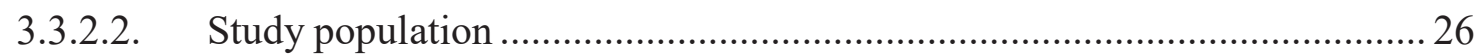

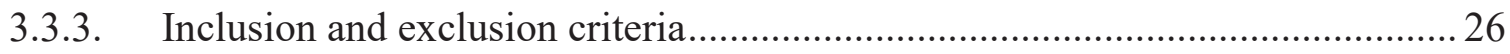

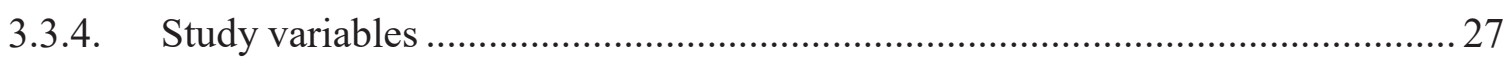

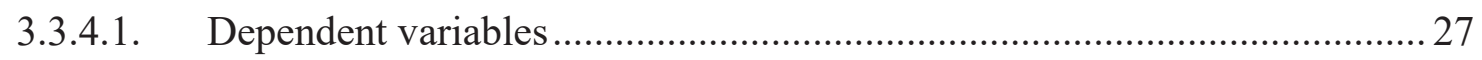

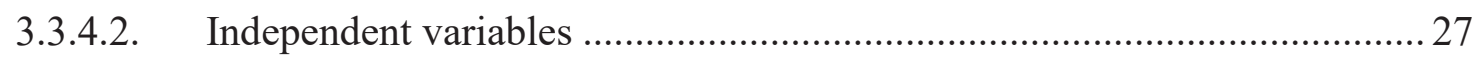

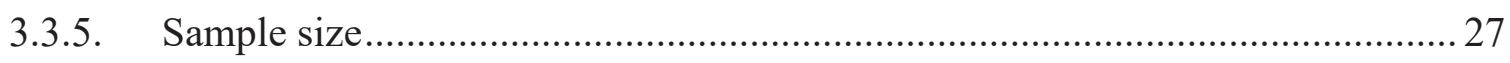

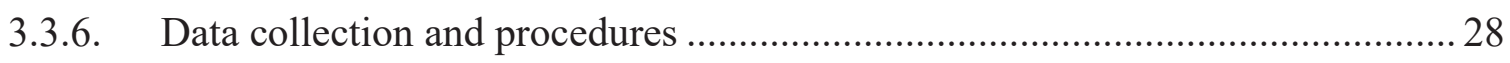

3.3.7. Development of data collection tools: questionnaire and data abstraction form 28

3.3.8. MRP identification, assessment and clinical significance................................ 29

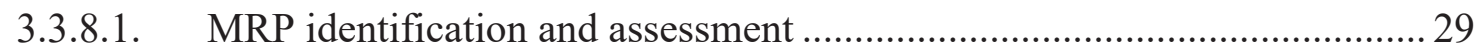

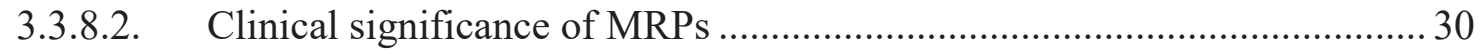

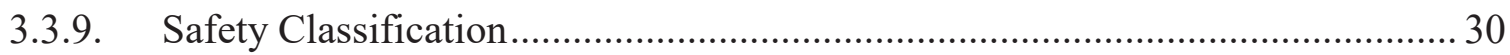

3.3.9.1. Safety classification of medicinal plants............................................... 30

3.3.9.2. Safety classification of conventional medicines .................................... 31

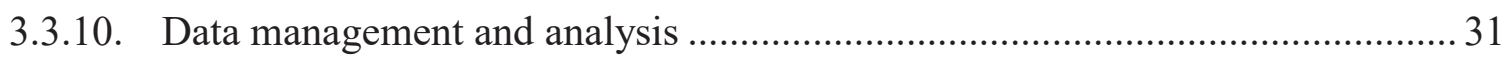

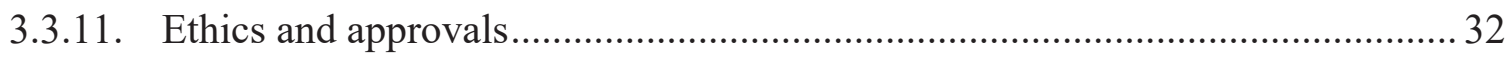

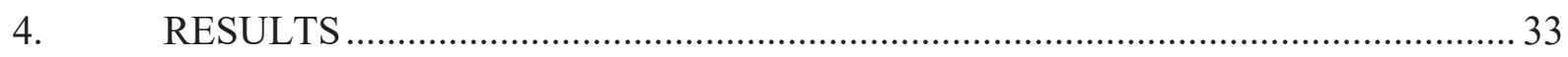


4.1. The use of medicinal plants by pregnant women in Africa: A systematic review .... 33

(Paper I)

4.2. Medicinal plants used among pregnant women in a tertiary teaching hospital in Jimma, Ethiopia: a cross-sectional study (Paper II)

4.3. Self-Medication and safety profile of medicines used among pregnant women in a tertiary teaching hospital in Jimma, Ethiopia (Paper III) 35

4.4. Medication-related problems among hospitalized pregnant women in a tertiary teaching hospital in Ethiopia: A prospective observational study (Paper IV)..................... 36

5. DISCUSSION

5.1. Summary of findings

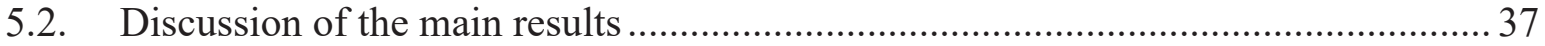

5.2.1. Prevalence of use of medicinal plants and pharmaceutical medicines............... 37

5.2.2. Reasons for use of medicinal plants and conventional medicines .................... 40

5.2.3. Utilization pattern of medicinal plants .............................................................. 41

5.2.4. Safety concerns of treatment modalities used by pregnant women ................... 42

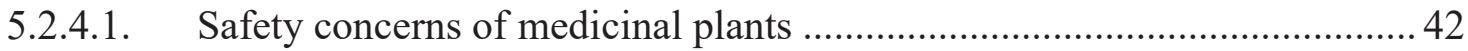

5.2.4.2. Safety concerns and MRPs of conventional medicines ............................. 43

5.2.4.3. Concomitant use of treatment modalities ............................................ 45

5.2.5. Factors associated with use of treatment modalities and MRPs ....................... 47

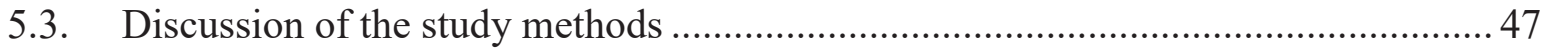

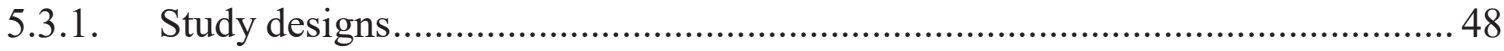

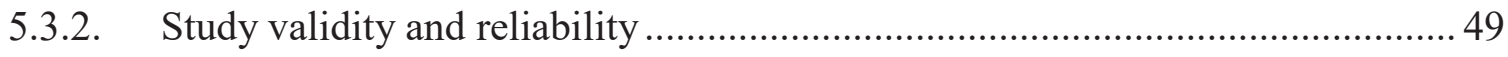

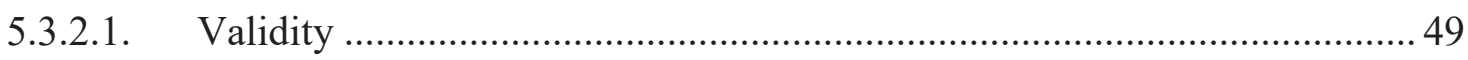

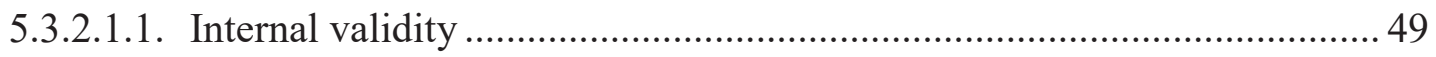

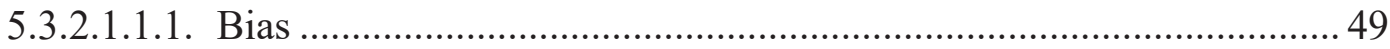

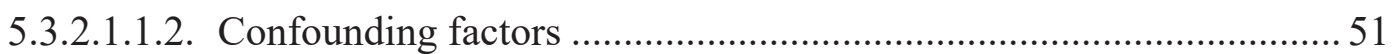

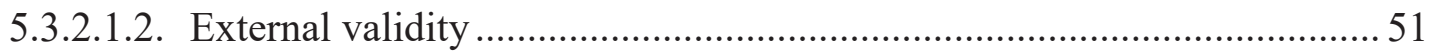

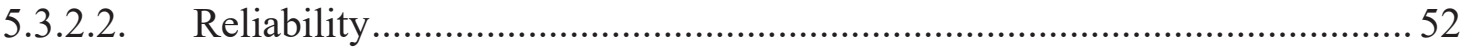




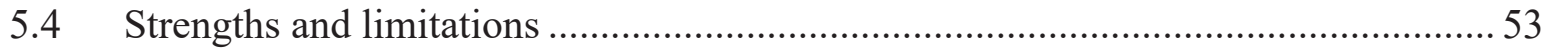

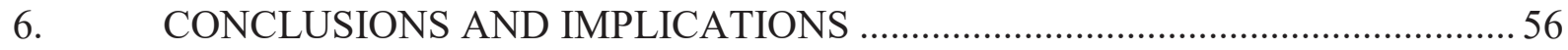

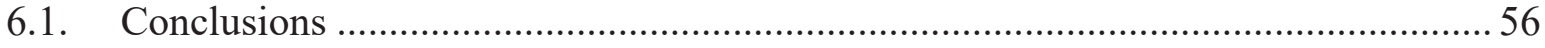

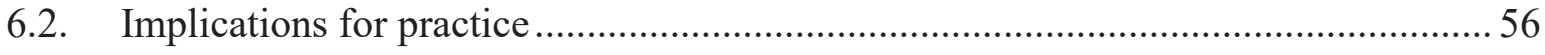

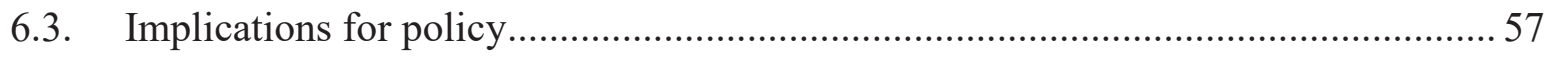

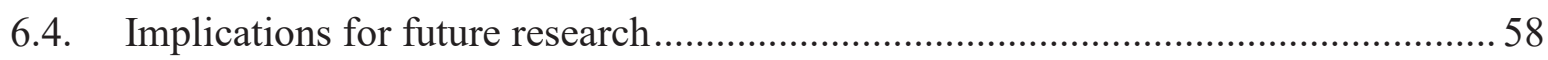

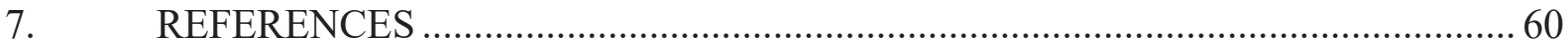




\section{Acknowledgements}

This PhD study was a long journey. Many individuals and institutions have contributed for the successful completion of this dissertation, without whom it might not have been possible.

I would like to extend my foremost gratitude to all the pregnant women who sacrificed their time, willingly participated, provided the necessary data, and made this work possible. A special acknowledgement also goes to University of Oslo for giving me the opportunity to pursue my $\mathrm{PhD}$ study. I am very grateful to the Norwegian State Educational Loan Fund (The Quota Scheme) for providing financial support for this study. The Norwegian $\mathrm{PhD}$ School in Pharmaceutical Sciences also deserves recognition for the travel grant assistance for the data collection. I also owe a debt of gratitude to Jimma University for granting me the study leave.

My esteemed supervisors Dr. Fekadu Abebe, Professor Johanne Sundby and Dr. Yesuf Ahmed were instrumental to the smooth undertaking of the $\mathrm{PhD}$ project. My sincere gratitude goes to Dr Fekadu Abebe, for his wholehearted encouragement, constructive feedback and for patiently guiding me throughout this journey. I thank you for your all-round support. I extend my deepest gratitude to Professor Johanne Sundby for her scientific guidance, unreserved encouragement, unconditional support and mentoring that provided me with energy and enthusiasm to complete this dissertation. I am extremely grateful for her unwavering support and belief in me from the beginning to the end of my $\mathrm{PhD}$ work. I would also like to thank Dr. Yesuf Ahmed for his concrete and helpful advice throughout my study. My thanks also go to my co-authors Professor Hedvig Nordeng and Professor Hugo J. De Boer for their invaluable comments and suggestions for the articles included in this dissertation.

I am indebted to Ibrahimu Madala for his indispensable statistical consultation during data analysis. I would like to acknowledge Associate Professor Emerita Nancy Eik-Nes for her English proofreading this dissertation. I also wish to thank Dr. Ahmed Madar for his review and suggestions for editing of the dissertation.

I am thankful for all data collectors, my friends and colleagues at Jimma University who assisted me during data collection. I truly appreciate your help. I am also truly grateful to all my $\mathrm{PhD}$ colleagues and friends at $\mathrm{UiO}$ with whom shared experiences, joy, and fun along this journey, the list is long and I truly value our friendship. 
I am also greatly indebted to the helpful people at the Department of Community Medicine and Global Health (UiO) including Morten Ariansen, Kåre Moen, Tone Kristin, Gry Stubberud, Line Løw and all the staff at the department for their help during my study in the department. I am also thankful to the current (Mariana Larsen Hatzianastasiou) and former (Michele Jeanette) Senior Executive Officers at the international education office for their kind support and full-hearted service when I was in need of their help.

I would like to offer my special thanks to Bezawit Temesgen, my office mate and friend, I am grateful for the chance to get to know you. Your friendship, encouragement and all-round support have been decisive for the successful completion of this $\mathrm{PhD}$ work.

Ms. Tone Gulbrandsen, I do not have the words to thank you enough, honestly no words at all! You have a special place in my and my family's heart forever.

My profound appreciation goes to Nuredin Nassir and Mejida Muhidin, for making my family and me to feel as if we are at home. We will always remember your kindness and hospitality.

In a special way, I would like to acknowledge Kidus Yohannes. When there was one thing after another with me, your encouragements, wholehearted support and valuable advice were unforgettable. I thank you from the bottom of my heart.

I would like to thank all my friends in Oslo for their care, kindness and help, particularly Memhru Melkamu deserves mention for his warm welcome when I first arrived in Oslo and for his compassionate motivation during my study. I am also sincerely grateful to Yordanos Hannes for her encouragement and being there whenever I needed her help.

My special and utmost gratitude goes to my beloved wife Jema Abdella Siraj for her unconditional love, patience, and endurance during my PhD study. Your encouragement and emotional support kept me going and prevailed at times when life was dumping me out of the frying pan into the fire. I would not have reached this stage without your endless support.

My sweet children, Aimal and Hamna, were my constant source of happiness and inspiration even at times when I have been so sad and almost desperate. In order for this day to come, you 
sacrificed many things especially the care of your dad. I hope you will forgive me when you grow up and understand that education is the key to everything. My beloved daughter Aimal, the time when we will sit together, cheer and enjoy our life is not far away. Know that I love you very much!

My family members back home, your immense help and encouragement during the $\mathrm{PhD}$ journey is highly appreciated. Zehara and Aliya, without your nurturing, neither would Aimal have grown to this stage nor would I have progressed in my academics. You are amazing aunties to my daughter. I thank you a million times!

I am grateful to my mother Tobiyaw Mohammed who taught me the value of hard work and the stamina to fight for my goals and ambitions. I will be forever grateful for your strength and determination.

Above all, all praises and thanks belong to the Almighty Allah - The One and Only- for His guidance and for giving me wisdom and strength throughout my studies!

Seid Mussa Ahmed

Oslo, March 2021 


\section{List of publications}

This dissertation is based on the following publications

I. The use of medicinal plants by pregnant women in Africa: A systematic review. Ahmed SM, Nordeng H, Sundby J, Aragaw YA, de Boer HJ. J Ethnopharmacol, 2018; 224: 297-313. doi: 10.1016/j.jep.2018.05.032.

II. Medicinal plants used among pregnant women in a tertiary teaching hospital in Jimma, Ethiopia: a cross-sectional study. Ahmed SM, Sundby J, Aragaw YA, Nordeng H. BMJ Open [submitted, manuscript ID: bmjopen-2020-046495.R1].

III. Self-medication and safety profile of medicines used among pregnant women in a tertiary teaching hospital in Jimma, Ethiopia: a cross-sectional study. Ahmed SM, Aragaw YA, Sundby J, Abebe F. Int J Environ Res Public Health 2020, 17(11), 3993; https://doi.org/10.3390/ijerph17113993.

IV. Medication-related problems among hospitalized pregnant women in a tertiary teaching hospital in Ethiopia: A prospective observational study. Ahmed SM, Sundby J, Aragaw YA, Nordeng H. BMC Pregnancy Childbirth 20, 737 (2020). https://doi.org/10.1186/s12884-020-03433-6.

The publications are referred to in the text by their Roman numerals. 


\section{List of Acronyms \& Abbreviations}

AIDS Acquired Immunodeficiency Syndrome

ANC Antenatal Care

ATC Anatomical Therapeutic Chemical classification system

CI Confidence interval

EDHS Ethiopian Demographic and Health Survey

FASS Farmaceutiska Spesialiteter i Sverige

FDA United States of America Food and Drug Administration

FMOH Federal Ministry of Health

HEP Health Extension Programme

HEWs Health Extension Workers

HIV Human Immunodeficiency Virus

IRB Institutional Review Board

JUMC Jimma University Medical Center

MDGs Millennium Development Goals

MMR Maternal mortality ratio

MRP Medication related problem

NCDIs Non-communicable Diseases and Injuries

NCDs Noncommunicable Diseases

NVP Nausea and vomiting in pregnancy

OR Odds ratio

OTC Over-the-counter

PHCU Primary Health Care Unit

$\mathrm{Rh} \quad$ Rhesus

RHBs Regional Health Bureaus

SDGs Sustainable Development Goals

SPSS Statistical Package for Social Scientists

TGA Therapeutic Goods Administration

WHO World Health Organization 


\section{List of Figures}

Figure 1: Map of the Federal Democratic Republic of Ethiopia

Figure 2: The town Jimma where Jimma University Medical Center is located 


\section{(Operational) Definition of key terms}

At least one social drug use: the use of at least one of the three social drugs alcohol, khat, or tobacco during pregnancy.

Concomitant use of medicines: the use of medicinal plants and pharmaceutical medicines together or one after the other during pregnancy to treat the same or different illnesses. Efficacy: the capacity of a treatment modality to produce the desired therapeutic effect. Isihlambezo: a herbal decoction of several medicinal plants used among the Zulu women in South Africa as a preventative health tonic during pregnancy.

Medicinal plant: a plant (wild or cultivated) that possesses therapeutic properties or exerts beneficial pharmacological effects on the human body and is used for medicinal purposes. It includes plants or plant parts (non-formulated crude drugs), plant preparations that contain parts of plants or combinations thereof as active constituents. In this dissertation the terms herbal medicine, botanical medicine and herb are used interchangeably when referring to medicinal plants.

Mitch: a culturally common febrile illness in Ethiopia which is believed to be caused by excessive sunlight and manifested by swelling and/or formation of sores on parts of the body. Pharmaceutical medicine: prescribed and non-prescribed medicines that are available in every modern health facility or drug retail outlet in which medical doctors and other healthcare professionals (such as health officers, nurses, and pharmacists) prescribe or dispense to treat symptoms and diseases of human beings in Ethiopia. In this dissertation the terms, conventional medicine, modern medicine, orthodox medicine, medicine, medication, drug, mainstream medicine and western medicine are used interchangeably when referring to pharmaceutical medicine.

Safety: the freedom from potential adverse effects related to the administration of a treatment modality.

Self-medication: the selection and use of conventional medicines by individuals to treat selfdiagnosed disorders or symptoms, or the intermittent or continued use of a prescribed medicine for chronic or recurrent diseases or symptoms on their own initiative Social drug: a term that refers to alcohol, khat, and tobacco. Unless and otherwise specified as "social drug", the term "drug" denotes pharmaceutical medicine.

Treatment modality: the term "treatment modality" in this study refers to both pharmaceutical medicine and medicinal plants. 


\section{Summary}

Background: In addition to the unique nature of pregnancy and the resulting physiologic changes, many women will experience different medical conditions. Consequently, they may use one or more types of medicinal plants and pharmaceutical medicines to relieve themselves from complaints, symptoms or illnesses. Inaccessibility and unaffordability of health care facilities as well as cultural acceptability of indigenous remedies may force women in Ethiopia and Africa at large, even worldwide, to rely on medicinal plants for their basic healthcare, a practice which also extends into pregnancy. However, use of medicinal plants in pregnancy presents a special challenge due to the scarcity of safety and efficacy information. There is also insufficient safety data on pharmaceutical medicines used in pregnancy. Pregnant women also use pharmaceutical medicines, prescribed by doctors or bought over the counter, once admitted to hospital, and therefore there is risk of medication therapy problem posing maternal and foetal risk. There is a lack of studies about prevalence of use, utilization and safety of the medicinal plants and pharmaceutical medicines among pregnant and lactating women in Ethiopia. This also complicates monitoring and regulating their use which in turn compromises pregnancy outcomes.

Objective: The overall aim of this dissertation is to study the prevalence, utilization, predictors and safety of medicinal plants and pharmaceutical medicines used among pregnant and lactating women in Jimma University Medical Center (JUMC) in Southwest Ethiopia.

Subjects and methods: This dissertation is based on a systematic review study which was performed from July 2016 to January 2017 (Paper I) and a hospital based cross-sectional study which was conducted at JUMC in Ethiopia from February to June 2017 (Papers II-IV). A systematic review of medicinal plants used in pregnancy in Africa was performed and the final systematic examination resulted in 50 scientific papers eligible for inclusion into the final review (Paper I). The next three papers are based on a cross-sectional study of medicinal plants (Paper II) and pharmaceutical medicines used and associated medication treatment-related problems in pregnant and lactating women at JUMC (Papers III, IV). A pre-tested intervieweradministered structured questionnaire and data extraction form were used for data collection in the hospital based study (Papers II-IV). 1117 consecutive pregnant or lactating women hospitalized in the maternity and gynaecology wards, who consented and fulfilled the inclusion criteria, were enrolled into the study. The same group of women comprised the study 
participants in all the three hospital based studies. Medication pregnancy risks were evaluated using US American and Australian risk classification systems, while medicinal plant safety was assessed using available scientific evidence.

Main Results: The systematic review revealed that the prevalence of medicinal plant use during gestation in Africa ranged from 2\% to $100 \%$. Similarly, the prevalence of concomitant use of medicinal plants and pharmaceutical medicines ranged from $2.4 \%$ to $77.3 \%$. A total of 274 different medicinal plants were used to manage pregnancy illness, Zingiber officinale Roscoe. (ginger) being the most widely used plant for relief of nausea and vomiting during pregnancy (NVP). Z. officinale is also the most studied plant and is relatively safe to use in pregnancy. Diverse utilization patterns were reported; however, in almost all the analysed works, the dose and the frequency of administration of medicinal plants were not indicated (Paper I).

The hospital based study indicated that $28.6 \%$ of the women had used medicinal plants during pregnancy. Linum usitatissimum L. (flaxseed) being the most commonly used (77.1\%) medicinal plant, and induction or shortening of labour $(60.2 \%)$ being the most common reasons for use. Wide-ranging utilization patterns were reported including oral route $89.7 \%$, one waterglass dose $47.5 \%$, once per day frequency $54.8 \%$, and "entire pregnancy" duration of treatment $32.9 \%$, among others. Only 5 of the 27 medicinal plants used by women were categorized as safe to use during pregnancy. Scarcity of health facility in a nearby area, hospitalization in the maternity ward, khat chewing, alcohol consumption, chronic illness, history of conventional medicine use and secondary school education were predictors of medicinal plants use during pregnancy $(\mathrm{P}<0.05$; all are at 95\% CI; adjusted OR range: 1.54 - 6.92) (Paper II). In total $27.0 \%$ of the expectant mothers self-medicated with one or more conventional medicines, largely analgesics $92.3 \%$. Furthermore, $36.7 \%$ of the self-medicated women had concomitantly used medicinal plants. Three-fourths of the conventional medicines used for self-care were classified as probably safe, whereas over one in ten as potentially risky to use during pregnancy. Almost $10 \%$ of women either chewed khat or drank alcohol during pregnancy. Medicinal plant users and Islam or Christian Orthodox religion adherents were significantly more likely to selfmedicate during pregnancy $(\mathrm{P}<0.05$; all are at 95\% CI; adjusted OR range: $1.78-2.22)$. On the other hand, access to a health facility close to residential area was inversely related to selfmedication ( $\mathrm{P}<0.05 ; 95 \% \mathrm{CI}$; adjusted OR 0.62) (Paper III). About 9 out of 10 women used 
at least one prescription or non-prescription western medicine in pregnancy whereas $24.3 \%$ women concomitantly used medicinal plants and conventional medicines prior to admission.

This study found that $28.9 \%$ of pregnant women encountered at least one medication related problem (MRP) and almost eight out of ten MRPs were considered to be of moderate to high severity. On the whole $41.9 \%$ of the total MRPs and $47.8 \%$ moderate to high severity MRPs were due to iron supplementation. The need for additional medication therapy $73.1 \%$ was by far the most frequent cause of MRP. Medication use before hospitalization, chronic disease, nulliparity and multiparity were significantly associated with occurrence of MRPs $(\mathrm{P}<0.05$; all are at 95\% CI; adjusted OR range: 1.91 - 2.38) (Paper IV).

Conclusion: Medicinal plants and conventional medicines (prescription and non-prescription medicines) are commonly used in pregnancy separately or concomitantly in Africa at large and in Ethiopia. Unless due care is taken, co-ingestion of treatment modalities could result in interaction and in turn negative pregnancy outcomes. Z. officinale is the most commonly used medicinal plant in many African countries mainly for the relief of NVP whereas $L$. usitatissimum is the medicinal plant most commonly used in Ethiopia, mainly for induction or to reduce the intensity and shorten duration of labour. Furthermore, the study findings indicate that medicinal plant utilization is variable and without standardization and thus may cause harmful foetal and maternal effects. Most of the conventional medicines used by pregnant women in Ethiopia were probably safe to use; however, the majority of the medicinal plants women used were either potentially harmful or did not have sufficient safety information to use in pregnancy. Moderate to high severity MRPs are a common phenomenon at JUMC maternity and gynaecology wards, predominantly in connection with absence of iron supplementation. Ensuring affordable access to health facilities and essential medicines, and creating awareness about appropriate use of various treatment modalities among pregnant women, healthcare professionals and other stake holders are vital for improved maternal healthcare. Pharmaceutical law enforcement as well as compliance to the national and international treatment, prescribing and dispensing guidelines in pregnancy care is essential to optimize therapy and prevent pregnancy harms. Finally, scarcity of safety data and proper documentations of treatment modalities used in pregnancy calls for more nationwide, multicenter and multilevel research. 


\section{INTRODUCTION}

\subsection{Medicinal plants and pharmaceutical medicines used in pregnancy}

\subsubsection{Medicinal plants used by pregnant women}

Medicinal plants are the most widely used traditional treatment in Africa and are used by $70 \%$ to $80 \%$ of the population for basic health services [1]. Over one third of Africans lack access to essential medicines, and healthcare facilities are scarce $[1,2]$. Due to a number of challenges connected with accessibility and affordability of health facilities and medicines, women are recognized to be the major users of medicinal plants for treatment of disease and maintenance of health [3-5]. This widespread use also extends into gestation to treat pregnancy induced illnesses, promote healthy pregnancy, ensure a normal birth, and promote and maintain health [3-6].

According to the World Health Organization (WHO) traditional medicine refers to "the knowledge, skills and practices based on the theories, beliefs and experiences indigenous to different cultures, used in the maintenance of health and in the prevention, diagnosis, improvement or treatment of physical and mental illness"' [1]. Medicinal plants are defined as " plants that possess therapeutic properties or exert beneficial pharmacological effect on the human or animal body'” [7].

A significant majority of Ethiopia's population rely on traditional medicine for basic healthcare [8]. Ethiopians have used traditional medicines for many centuries, and this use has become an integral part of the different cultures in the country $[9,10]$. Until the introduction of western medicine, it was the only medical care for the people of Ethiopia [11]. Even now, the vast majority of Ethiopia's population, around $80 \%$, rely on traditional medicine for primary health care $[12,13]$, out of which more than $95 \%$ are medicinal plants [13]. Indigenous healthcare practices are also as diverse as the various cultures [11]. Various categories of traditional medicine providers including herbalists, bonesetters, spiritual healers, traditional birth attendants and tooth extractors have been serving the wider population for centuries in the country $[8,14]$.

Ethiopia is home to a diversity of biological resources including about 7000 higher plant species, of which 650 to 1000 species are used as medicinal plants [12], most of them are confined to south-western Ethiopia [15-17]. Furthermore, the lion's share of the forest area of 
the country is found in this region [18]. In addition, medicinal plants are used to varying extents in conjunction with pharmaceutical medicines in the country [12]. Although medicinal plants, like any traditional medicines, are not regulated in Ethiopia [12], the National Drug Policy of the country covers both medicinal plants and conventional medicines [14]. Even though most of the population depend on medicinal plants for basic health care, there is no regulatory requirement for the sale or safety assessment of medicinal plants. Moreover, medicinal plants are not included in the national essential medicines list of Ethiopia [8].

Medicinal plants are often viewed as natural and safe to use among child bearing women [3, 19]. Nevertheless, there are limited human data on their safety in pregnancy [3, 20] and studies stress that even some medicinal plants have harmful constituents which may cause severe negative consequences both to the mother and unborn child [3, 21-25]. Hence it should not be presumed that a treatment is safe for use during pregnancy because it is natural $[21,22]$. Thus far Z. officinale (ginger) is the only medicinal plant that can be recommended for NVP based on human studies $[3,20]$. The fact that medicinal plants are available without the direct observation of health care professionals underscores the importance of cautious use among anticipating women $[3,6]$.

Only limited studies have investigated the prevalence of medicinal plant use among anticipating women in the African continent [3]. Those that do show a wide range of use, from $1 \%$ to $100 \%$ [3]. In Ethiopia, it ranges from $2.0 \%$ to $73 \%$ [3]. Studies also indicated that medicinal plant use is growing among pregnant women in the western world with prevalence ranging from $0.9 \%$ [26] to $96.0 \%$ [27].

\subsubsection{Pharmaceutical medicines used by pregnant women}

The fact that certain drugs given to gravid women may prove harmful to the developing foetus is one of the classical problems in pharmacological therapy [21]. Despite the absence of randomized controlled trials to guide their use in pregnancy, more than nine out of ten expectant mothers take at least one conventional medicine; a prescription or over-the-counter (OTC) medicine [20]. Avoiding all medicines during pregnancy is not possible because women may enter gestation with medical problems that require ongoing and episodic therapy; they may develop new health problems and old ones can be worsened necessitating pharmacological therapy $[6,21,28]$. Besides, disorders unrelated to but requiring pharmaceutical management may emerge during gestation, such as urinary tract infections, upper respiratory infections, and 
gastrointestinal upsets to name a few [21]. Discontinuing treatments, for example for asthma, diabetes, HIV/AIDS, hypertension and psychiatric disorders can have profound long-term health implications not only to the woman but also to the unborn child $[6,21,28,29]$. In addition, minerals such as iron, vitamins and dietary supplements are crucial for the health of the gravid women and developing foetus [21]. Women who are pregnant may also be exposed to many other agents that may have an adverse effect on foetus [21]. On the other hand, excessive use of medicines can have potential negative consequences for the health of the woman and the unborn child [6]. It therefore becomes important to examine the pattern of medicine use in pregnancy to see to what extent there may be room for improvement in the light of current knowledge [21].

Generally, medicines or substances an expectant mother takes can affect the foetus in several ways $[21,30]$. They can act directly on the foetus causing damage or abnormal development leading to birth defects or death $[21,22]$. They can also alter the function of the placenta, usually by constricting blood vessels and reducing the blood supply of oxygen and nutrients to the foetus from the mother; this may result in a baby that is underweight and underdeveloped [21, 30]. Moreover, some substances can cause the muscles of the uterus to contract forcefully; indirectly injuring the foetus by reducing the blood supply or triggering pre-term labour and delivery $[21,22]$.

In general, unless absolutely necessary, drugs should not be used during pregnancy because drugs taken by a gravid woman can reach the foetus and harm it by crossing the placenta, the same route taken by oxygen and nutrients, which are needed for the growth and development of the foetus [22].

\subsubsection{Self-medication}

According to the WHO's definition, "self-medication is the selection and use of medicines by individuals to treat self-diagnosed disorders or symptoms, or the intermittent or continued use of a prescribed medicine for chronic or recurrent diseases or symptoms" [31]. In most underdeveloped nations where health systems are weak and health workers are scarce [31, 32], expectant mothers prefer to self-medicate first as an accessible and affordable alternative and look for professional medical help only if the condition fails to respond, persists or becomes more severe [31-33]. 
Self-medication is an important component of healthcare system and its practice is widespread in both developed and developing countries $[31,34,35]$. It is widely practiced in the African continent $[36,37]$. In most developing nations, including Ethiopia, the majority of healthrelated problems or symptoms experienced by individuals, nearly $60 \%-80 \%$, are either tolerated or treated through self-medication as a lower-cost alternative [34].

The practice of self-medication varies in different communities and is influenced by a number of factors $[32,34,37]$. The key drivers of self-medication in less developed countries like Ethiopia are scarcity or unaffordability in the healthcare delivery systems; the situations include such aspects as long waiting times in the health institutions, inadequacy of health personnel and healthcare facilities, significance attributed to the disease, previous experiences with the symptom or illness, high costs, and patients' attitudes toward healthcare providers [33-35, 37]. Weak legislation regulating dispensing and sale of pharmaceuticals, and their easy availability outside the formal and authorized institutions also contribute to increased rates of selfmedication [33, 37-39]. In addition, sociodemographic factors and lifestyle also contribute their share in self-medication practice [32, 34, 37]. Consequently, consumers prefer to manage their common health problems using self-medication because it is an accessible, cost effective, and time efficient option [31,33].

The WHO has indicated that responsible self-medication has a positive social, economic and health benefits, particularly in the care of minor illness [31]. Self-medication also helps people to play an active role in their own health care and makes them more health-conscious [31, 34]. It can help to prevent and treat ailments and conditions that do not require medical consultation and provides affordable alternative for handling health problems [31, 34]. Moreover, it can save time spent in visiting health facilities and even lives in acute conditions, and may contribute to reduce health care costs [34].

Despite its obvious benefits, self-medication can cause substantial problems for the individuals and the community, especially in pregnant women [33, 40, 41]. Self-medication may lead to wastage of resources and antimicrobial resistance. It may also cause a number of potential health-related hazards such as misdiagnosis or masking potential health problems, delay in treating of serious medical conditions, polypharmacy, drug-drug interactions, drug-herb interactions, adverse medicine reactions, prolonged suffering, medicine dependence, among others $[34,35]$. To make it worse, WHO reports that in many developing countries a large 
number of prescription-only medicines are, in fact, widely retailed without a prescription [31]. In Ethiopia, the purchase and use of prescription-only medications including antimicrobial agents without medical consultation is reported to be alarmingly high [42]. Severe difficulties related to antimicrobial self-treatment such as low dose, short duration of treatment, discontinuing treatment too early, using leftover medicines, sharing of medicines, and therapeutic failure were also reported [35].

Expectant mothers are particularly prone to self-medication, since pregnancy involves hormonal and physiological changes that can cause nausea, vomiting, indigestion or heartburn, constipation, pain, headache, common cold, cough, dizziness and fever, that can cause the pregnant woman to increase her use of medicines [33, 40, 41, 43]. However, self-medication during gestation can increase the risk of congenital malformations and endanger maternal and foetal health $[32,40]$.

Globally, the prevalence of self-medication among pregnant women varies from $1.9 \%$ to $92.6 \%[32,33]$. It is also common in Sub-Saharan African nations with prevalence ranging from $1.9 \%$ to $62.9 \%$ [36, 37, 44-46]. In Ethiopia, the prevalence of self-medication with conventional medicines in pregnancy has been shown to range from $1.9 \%$ to $29.1 \%$ [28, 47]. In particular, the Ethiopian population has low health literacy and the ordinary user will usually have no specialized knowledge of the principles of therapy, or of the specific characteristics of the conventional medicines used leading to inappropriate use [31].

Self-medication in expecting mothers is common in many African nations [33], potentially exposing the unborn child to medicines that may have risk [44]. Use of potentially risky medicine by expectant mothers has been reported, with prevalence estimates of $49.5 \%$ in Africa [44] and $28.0 \%$ in Europe [48]

\subsubsection{Safety of medicines in pregnancy}

A common concern in the medical care of pregnant women is the fear that certain medicines taken by the mother can cross the placenta, reach the foetus and pose a teratogenic threat $[6,21$, 30]. Before marketing a new drug, the manufacturer almost never tests the product in gestating women to determine its effects on the unborn child. Consequently, most drugs are not labelled for use among pregnant population [49]. Despite the absence of randomized controlled trials 
for safety in pregnant women, more than nine out of ten pregnant women take a prescription or OTC medication [20].

The 1960's thalidomide tragedy that resulted in malformed extremities in almost 10000 children $[6,50]$ and the 1971 's diethylstilboestrol linked teratogenic effects $[21,50]$ were tragic examples of the use of harmful medications. The lesson that certain drugs taken during the gestational period may have deleterious effects to the unborn child changed forever the perception of pregnant women and their healthcare professionals [21]. Therefore, medical care should always maintain a fine balance that no harm should be posed to the unborn baby due to the medicine, and no harm should come to the mother or foetus due to inadequate treatment $[36,49]$.

\subsubsection{Pregnancy risk classification for medicines}

Mindful of the fact that medicines are a risk to the foetal and an estimated 10 percent or more of birth defects result from maternal medicine exposure [51], different nations have established agencies that assign a risk category to each medicine used during pregnancy according to their foetal safety information [6]. The most well-known are the U.S., the Australian and the Swedish pregnancy risk classification systems [6]. These risk classes apply only to recommended doses [30] and the safety profile of some medications may change according to the trimester of gestation [51].

The Swedish system for the classification of foetal risk of drugs (Farmaceutiska Spesialiteter i Sverige [FASS]) was introduced 1978 [52] and was the first of its kind [53]. It classified drugs used in gestational period in 4 general categories, A (Reliable clinical data indicate no evidence of disturbance of the reproductive process) to $\mathrm{D}$ (human data indicate an increased incidence of malformations) $[52,54]$. FASS further categorized category B to 3 subgroups B1, B2, and B3 based on animal data [52].

In 1979, the United States of America Food and Drug Administration (FDA) developed a system that determines the teratogenic risk of prescription and OTC medications by considering the quality of data from animal and human studies [55]. The FDA classifies drugs used in pregnancy into five-letter categories (from A to D and X) [55]. Category A is considered the safest and category $\mathrm{X}$ is absolutely contraindicated in pregnancy [55]. This provides therapeutic guidance for the clinician $[6,21]$. 
Similarly, the Therapeutic Goods Administration (TGA) of the Government of Australia have developed and used a system to determine the pregnancy risk category for each medicine since 1989 [56]. The Australian categorisation system classifies medicines in to 7 categories; category A medicines are considered to be safe for use in gestation, category B1, B2, B3 medicines have not been shown to increase the frequency of deformity or harmful effects on the human foetus; category $\mathrm{C}$ may cause harmful effects on the human foetus without causing malformations [56]. Category D drugs have foetal risk, but benefits may outweigh the risk and Category X drugs are strictly prohibited in pregnancy [56].

The definitions used by the different classification systems differ considerably and place drugs in different risk groups $[53,54]$. This makes it difficult for women and health care providers to decide whether or not to use medications during pregnancy [21, 29]. In response to the criticism of the imprecise and simplistic nature of the five-letter system [20], in 2015 the FDA issued a new approach to labelling by replacing the former letter categorisation with narrative risk summary based on available data [55]. The new guideline divides information into pregnancy and breastfeeding categories, each with the subcategories of risk summary, clinical considerations, and summary of relevant clinical information [20]. The European Union has also adopted a similar medicine labelling method [6].

Ethiopian standard treatment guidelines use the FDA five letter pregnancy risk categorization of medicines [57, 58].

\subsubsection{Medication related problems}

The goal of pharmacotherapy is to achieve an optimal therapeutic outcome. When the outcome is not optimal, a medication related problem (MRP) may have occurred [59]. An MRP is any unwanted event that involves or is suspected to involve a patient's medication treatment that actually or potentially interferes with attaining the anticipated optimum outcome of medical care and requires healthcare expertise judgment to settle [59-62]. Medication-related needs of a patient that have gone unmet give rise to a MRPs [60, 62].

When medications are used for the treatment of disease, the possible outcomes of therapy range from the intended beneficial effect to the unintended minor side effects and even loss of life $[59,63]$. Although many of the unintended events (i.e. MRPs) can be resolved without a negative effect on patient's health, some of them can be detrimental $[59,64]$ and if left 
untreated, may ultimately lead to medication-related mortality [63]. Studies suggest that except for some patient idiosyncratic unintended events, a large number of drug related problems are preventable [63].

In the United States, the estimated annual total cost of medication-related morbidity and mortality resulting from non-optimized treatment regimens was US \$528.4 billion (equivalent to $16 \%$ of total US health care expenditures) in 2016 [65]. The annual estimated mortality resulting from non-optimized medication therapy was 275689 deaths in 2016 [65].

Different classifications systems of MRPs have been proposed and described in the literature $[66,67]$. The majority of the classification systems support the assessment of the four drugrelated needs: indication, effectiveness, safety, and adherence [59, 60,62]. MRPs are commonly classified into seven general categories: unnecessary drug therapy, need for additional drug therapy, ineffective drug therapy, dosage too low, dosage too high, adverse drug reaction (ADR) and non-compliance [60]. Some authors have suggested additional miscellaneous subgroups [66, 68], including but not limited to need for additional laboratory test and incomplete drug order.

A number of factors have been implicated as being associated with the occurrence of MRPs including age, sex, polypharmacy, co-morbidity and the use of specific drugs (antithrombotics, antidiabetics) [68-70].

Globally, there is scarcity of research particularly investigating the extent and nature of MRPs in pregnant women $[68,69]$. Owing to the paucity of information on the safety of drugs in gestation, drug treatment of pregnant women often calls for balancing the associated benefits and risks to both the gravid women and unborn offspring [71]. Data gleaned from the studies available illustrate that the prevalence of MRPs among expectant mothers in an inpatient setting varies from $42 \%$ in Norway [69] to $83 \%$ in Australia [68]. Concerning the most common classes of MRPs in these studies, "Need for additional drug" (46.7\%) [69], “incomplete medications charted on admission" (28\%) [68], “dose too high" (26\%) [68], "adverse drug reaction" (20.0\%) [69], "incomplete drug order" (15\%) [68], and "patient adherence" (10.5\%) [69] were reported. The most frequent medication groups implicated in MRPs were medication for the alimentary tract and metabolism (31\%), medication for the nervous system, (30\%) [68], medication for the respiratory system (25\%) [69], anti- 
infectives for systemic use (18\%) [69] and medications acting on blood and blood-forming organs $(16 \%)[69]$.

MRPs have negative consequences on both patients and society including poor health outcomes, low productivity, reduced quality of life, increased hospital stay, increased overall medical care costs, increased risk of morbidity and mortality. In addition, it erodes public confidence in health systems $[63,69,72]$. Unless due care is taken in the patient care process, MRPs have the potential to cause patient harm, ranging from low to severe patient deterioration or discomfort [73].

The extent, frequency, type, and contributing factors of MRPs are of great importance to health care practitioners, administrators, patients, and society as a whole [63]. Therefore, identification, resolution, and prevention of MRPS is a matter of paramount importance [63].

\subsection{Social drugs used}

In addition to the safety concerns of various prescribed and non-prescribed medications used by pregnant women, caution should be given to other substances that can bring about risk to pregnant women $[21,74]$. Use of social drugs such as tobacco, alcohol and khat are major concerns in Ethiopia [75]. These social drugs are also well recognized risk factors for noncommunicable diseases (NCDs) in the country [76].

\subsubsection{Tobacco}

Over a fifth $(22 \%)$ of the world's adult population aged 15 years or older are estimated to be current tobacco smokers, including $36 \%$ of men and $8 \%$ of women [77]. WHO estimates that $10 \%$ of the African population aged 15 years and over are current tobacco smokers, including $17 \%$ of men and $3 \%$ of women [77]. Smoking and smoke exposure have potentially harmful health effects $[21,77]$. WHO estimates that tobacco use is responsible for almost six million deaths each year [77]. Unless strong action is taken to curb the tobacco epidemic, it is projected to claim the lives of eight million people per year by 2030 [77].

Tobacco use in gestation has adverse outcomes on women, the foetus and the baby at birth and throughout his or her early development [21, 35]. Maternal tobacco use, including use of smokeless tobacco, during gravidity is associated with increased risks for stillbirth, miscarriage, preterm labour, preterm birth, low birth weight, ectopic pregnancy, uterine infection, premature 
rupture of membrane, restricted foetal growth, abruptio placentae, placenta previa, and congenital anomaly [21, 77, 78]. After birth, the risk for sudden infant death syndrome is increased among babies of women who smoked during or after pregnancy [21, 77]. Therefore, expectant mothers need to be informed about the risk of smoking on the foetus, and encouraged to cease smoking during the prenatal, perinatal, and postnatal periods $[21,30]$.

According to a recent Ethiopian national report, cigarette smoking and use of any type of tobacco are rare in Ethiopia; less than 1\% of women and $4 \%$ of men smoke any type of tobacco [75]. However, institution studies have reported smoking as high as $5.0 \%$ in anticipating mothers in Ethiopia [79].

\subsubsection{Alcohol}

The WHO estimates that there were more than 3 million alcohol-attributable deaths globally in 2016 [80]. The prevalence of alcohol use in many Sub-Saharan African countries is high and reported to be increasing among women [80]. There is no safe type, amount, and time for drinking alcohol during pregnancy [80, 81]. Alcohol use during pregnancy is the foremost preventable cause of developmental disabilities and birth defects [80]. Alcohol use among women carrying a child is related to various adverse health risks for both the mother and foetus $[30,78,82]$. Adverse health effects are seen at low levels of alcohol starting at $10 \mathrm{~g}$ per day use [78]. Alcohol is a teratogen that can readily cross the placenta, resulting in damage to the brain and other organs of the developing embryo and foetus [81]. Its consumption in gestation has been associated with adverse outcomes, including low birthweight, spontaneous abortion, stillbirth, preterm birth, intrauterine growth retardation, congenital malformations and Foetal Alcohol Spectrum Disorder [30, 80, 82, 83]. Foetal Alcohol Syndrome is the most severe end of Foetal Alcohol Spectrum Disorder [21, 22, 82]. Global incidence of Foetal Alcohol Syndrome is estimated to be 1 per 2000 live births [21, 74]. This syndrome is linked with prenatal or postnatal growth restriction, birth defects and cognitive, behavioural, emotional, and adaptive functioning deficits $[21,22,80,82]$. Due to its severe negative impacts on health, many government bodies and agencies recommend women who are pregnant, or might soon become pregnant not to drink alcoholic beverages as the safest option [30].

The prevalence of alcohol consumption in the pregnant population in Sub-Saharan Africa varies widely, ranging from $4.3 \%$ to $59.3 \%$ [84]. In Ethiopia, the overall prevalence of alcohol 
consumption in pregnant women in varying frequencies and amounts ranges from $4.3 \%$ [84] to $39.8 \%[83]$.

\subsubsection{Khat}

Khat or qat or chat (Catha edulis Forsk) is a flowering plant native to east Africa and the Arabian Peninsula $[85,86]$. Khat chewing has been a social and cultural custom of people living in this part of the world for many centuries, mainly for its stimulant effects [85-87]. Globally, over 20 million people chew khat leaves daily, specifically in the khat belt countries that include Somalia, Djibouti, Ethiopia, Kenya and Yemen [86, 88].

Chewing fresh khat leaves is the most common mode of administration. Less frequently, dried leaves are used to make drinks and for smoking [85, 86]. Cathinone, an alkaloid structurally similar to that of amphetamine, is the main psychoactive constituent of khat and is mainly found in young shoots [85-87]. Acute administration of khat enhances mood and alertness; nevertheless, these symptoms are typically followed by discomfort including depression, anxiety, and insomnia [85, 87]. Chronic khat use has been reported to be associated with major physical, psychological, and psychiatric negative consequences [87]. Studies reported that chewing khat is linked to manic illness, psychosis, violent reactions, sleeplessness, nervousness, nightmares, anorexia, urinary retention, constipation, increase in suicidal depression and hallucinations $[85,86]$. Its use is also linked with systemic hypertension, increased heart rate, palpitation, sweating and cold peripheral extremities [86].

Human studies have found that khat use is a significant risk factor for gestation and delivery complications, which may contribute to infant mortality [85, 87]. Based on a study on pregnant guinea pigs, Jansson et al. have indicated that Khat chewing during pregnancy may impair foetal growth by decreasing placental blood flow [89]. Different studies have reported that chewing khat is associated with various obstetric effects including, but not limited to, restrictive dietary behaviour, anaemia, retarded foetal growth, stillbirths, low birth weight, inhibition of lactation, and perinatal and infant death $[85,86]$.

Khat is freely available and a highly valued export commodity in Ethiopia [90]. It is not illegal and is a social product consumed in many parts of the country [85]. Furthermore, as a local substance of social importance, the habit of chewing khat has become popular among all 
segments of the society, including expecting mothers [85, 90]. The prevalence of $k h a t$ chewing in pregnancy varies from $10.0 \%$ in southern Ethiopia [91] to $35.8 \%$ in south central Ethiopia [78].

Cognizant of the harmful health impacts substance use among the public, Ethiopia has ratified Proclamation No. 1112/2019 [92], which restricts alcohol and tobacco use, and prohibits the smoking of Shisha. The bill outlaws the sale of any alcoholic drink to anyone under the age of 21. It also requires the label of alcoholic drinks to contain a warning that alcohol consumption may cause health problems. It also prohibits pregnant women from drinking alcohol because of the risk of congenital abnormalities. The bill prohibits the sale or offer of tobacco products to any person under the age of 21, [92]. Nevertheless, nothing is mentioned about cigarette smoking and khat chewing in pregnancy.

\subsection{Maternal health in global perspective}

Maternal health is the foundation of a healthy and productive society [93]. According to WHO, "maternal health refers to the health of women throughout gestation, childbirth and the postnatal period [94].' It includes the health care dimensions of family planning, preconception, antenatal, childbirth and postnatal care in order to decrease maternal morbidity and mortality [95]. As maternal health and new-born health are closely linked [96], a healthy start during the perinatal period affects the rest of the life of the baby [97].

Although significant progress has been made in the past couple of decades, maternal death during pregnancy, childbirth and after delivery is still unacceptably high [94]. Most maternal deaths are preventable [94] and evidence shows that timely management and treatment can make the difference between life and death for the mother and the new born [97]. Worldwide, estimates for 2017 show that about 295000 women died from pregnancy or childbirth-related complications [96, 98]. The global maternal mortality ratio (MMR) in 2017 is estimated at 211 maternal deaths per 100000 live births [99]. Out of the total worldwide maternal deaths, 94\% occurred in developing countries [98], with Africa south of the Sahara alone accounting for roughly $66 \%$ (196 000) of maternal deaths [96, 98, 99].

Maternal death is the health indicator that shows the greatest difference between undeveloped and developed countries [96, 98]. A 15-year-old woman in Sub-Saharan Africa has a 1 in 37 risk of maternal death $[96,98]$. The risk for a 15-year-old woman eventually dying from a 
maternal causes in high income countries is 1 in $5400[96,98]$. The high levels of maternal mortality and morbidity in developing countries demonstrate the need for accessible and quality maternal health services [98, 100, 101] including skilled institutional care [102].

Although there is still a long way to go to achieve the Sustainable Development Goal-3 (SDG3) of 70 maternal deaths per 100,000 live births global target, there has been significant progress in MMR between 2000 and 2017 [96, 98]. In this period, Southern Asia achieved the greatest overall reduction in MMR, with a reduction of nearly 60\% (from 395 to 163 maternal deaths per 100,000 live births) $[98,99]$. Despite its very high MMR in 2017, the Sub-Saharan African region has also attained a significant decrease in MMR of nearly 40\% (from 878 to 542 maternal deaths per 100,000 live births) during this period [96, 98].

\subsection{Maternal health in Ethiopian context}

\subsubsection{Country profile and demography}

The Federal Democratic Republic of Ethiopia, Africa's oldest independent country [103, 104], is located in the center of the Horn of Africa [105]. The country is situated between the Equator and Tropic of Cancer, between $3^{0} \mathrm{~N}-15^{0} \mathrm{~N}$ and $33^{\circ} \mathrm{E}-48^{0} \mathrm{E}[102,106]$. The country covers an area of 1.1 million square kilometres ranging from 4,620 metres above sea level at Ras Dashen Mountain to 148 meter below sea level at the Danakil (Dallol) Depression [102, 107]. Ethiopia shares boundaries with Eritrea to the north and north-east; Sudan and South Sudan to the west; Somalia and Kenya to the south; Djibouti \& Somalia to the east [105]. Its capital and largest city is Addis Ababa $[102,105,108]$. Ethiopia is the most populous landlocked country in the world and the second-most populous country in Africa (after Nigeria) $[102,103,105$, 108] with over 109 million inhabitants as of 2018 [76], of which more than $80 \%$ are rural residents $[107,109]$. Approximately half $(45 \%)$ of the total population is below the age of 15 years, only $3 \%$ are in the age group of over 65 years, the sex ratio between males and females is almost equal and the average fertility is 4.1 births per woman [102].

Administratively, Ethiopia is divided into nine National Regional States and two administrative cities [110, 111]. The National Regional States and city councils are in charge of their own legislative and administrative functions, except for foreign affairs and defence [112]. The regional states and city administrations are further divided into Zones, Woredas (districts) and Kebeles (the smallest administrative unit) [102]. Agriculture is the backbone of the country's economy, accounting for $45 \%$ of the gross domestic product, $84 \%$ of the exports, and $80 \%$ of 
total employment $[109,113-115]$. It has a highly diverse population with different religions, languages and ethnic groups $[102,109]$. Gross national per capita income remains at $\$ 850$ United States Dollar in 2019 [116] despite the government pushing to reach lower-middleincome status by 2025 [115].

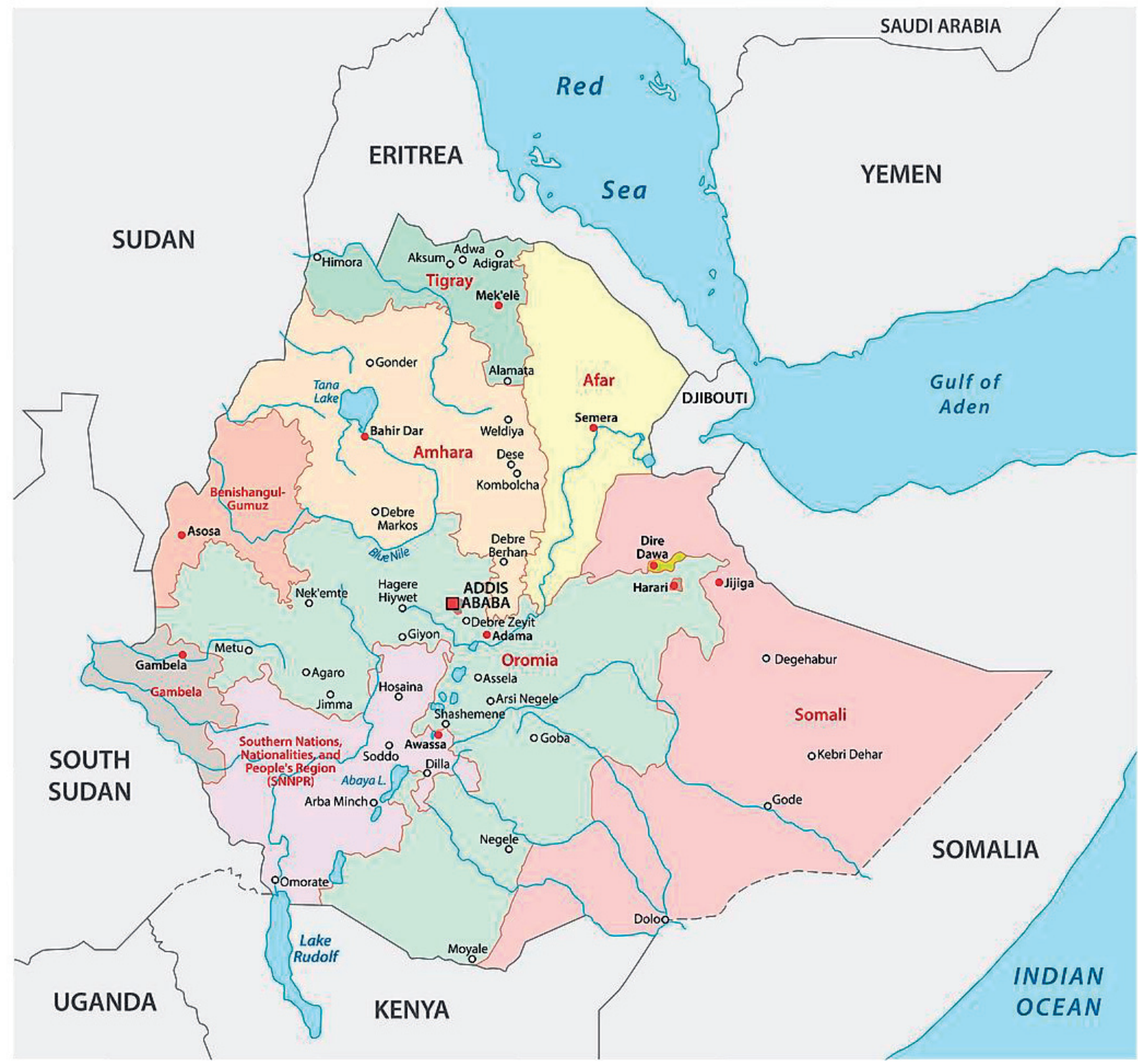

Figure 1: Map of the Federal Democratic Republic of Ethiopia

Source: https://www.worldatlas.com/maps/ethiopia

\subsubsection{Healthcare in Ethiopia}

Pregnant women use various levels of health care, before, during and after birth. In Ethiopia, inadequate access and underutilization of health facilities complicate maternal healthcare [117]. The Ethiopian healthcare is organized into a three-tier system to deliver essential health services and ensure referral linkages [107]: primary, secondary, and tertiary level of health care [118]. 
The first, at the woreda (district) level, is the primary healthcare unit (PHCU) [102, 119]. A PHCU is composed of a primary hospital that serves $60,000-100,000$ people; four health centers (each serving a population of $15,000-25,000$ ) and five satellite health posts are attached to each health center. Each health post provides service for 3,000 - 5,000 people in every Kebele (a cluster of villages) [102]. The second tier consists of a general hospital that serves 1 - 1.5 million people $[102,119]$. A general hospital provides inpatient and outpatient services and functions as a referral center for primary hospitals [107]. The third level comprises a specialized hospital that renders tertiary level healthcare for 3.5 - 5 million people $[102,119]$ and serves as a referral from general hospitals [107].

The three-tier public health service delivery system corresponds to the administrative structure of the government $[102,107]$. The Federal Ministry of Health (FMOH) manages and finances tertiary hospitals; The regional health bureaus (RHBs) manage and finance secondary hospitals, mostly regional hospitals; and The wored $a$ health offices manage and finance the primary health care units [107]. While the FMOH is in charge of formulating and harmonizing health programmes and strategies, the RHBs are mostly responsible for actual implementations [120].

The Declaration of Alma-Ata, which was adopted in September 1978, identified primary health care (PHC) as the key to the attainment of the "Health for All" goal around the globe [121]. The declaration called on all governments to include a PHC approach in their health systems [121]. The government of Ethiopia launched a community health programmes called health extension programme (HEP) in 2003 in the rural areas of agrarian regions, and then expanded to pastoral communities in 2006, and to urban areas in 2009 [107]. The overall goal of HEP is to create a healthy society and to reduce maternal and child morbidity and mortality rates $[107$, 122]. The programme empowers individuals and communities to manage their health problems $[102,107]$. It inspires families to produce and be responsible for their own health $[102,107]$. HEP has four health programmes: environmental hygiene and sanitation, disease prevention and control, family health services, and health education and communication [102, 107]. These areas of care correspond to the elements of primary health care coverage as specified in the Alma-Ata Declaration [121].

The Health Extension Workers (HEWs) are the key drivers of the programme [102]. HEWs are those who completed 10th grade and went through one year basic health promotion, disease prevention, selected curative services, and documentation of health information $[102,107$, 
123]. Each health post, located at the kebele level, is staffed by two government-salaried female HEWs $[102,107]$. At present, the HEP has deployed approximately 40,000 HEWs [102, 107, 123] and an estimated three million Women's Development Army volunteers across the country [123]. Over 16,000 health posts are providing basic healthcare service nationally [102]. HEWs refer patient to health centers when more complicated care is needed [107].

\subsubsection{Maternal health in Ethiopia}

Maternal and neonatal mortality rates in Ethiopia are among the highest in the world and are attributable to a range of socioeconomic, political, and demographic factors [75, 100, 124, 125]. Similar to the global trend [98], research findings indicate that haemorrhage, hypertensive disorders during gestational period, complications of unsafe abortion, obstructed labour, and sepsis are the leading causes of maternal deaths in the country [101, 102]. Complications during and after pregnancy and delivery are the main causes of maternal death and most of these complications are preventable or treatable [98]. The underlying causes for these deaths are poverty, inadequate, inaccessible, or unaffordable health care, unequal access to resources, low socioeconomic status of women and illiteracy [98].

Maternal and child health is one of the main concerns of Ethiopia $[102,126]$. With the aim of reducing the high maternal death rate, the country executed a set of high impact interventions such as antenatal care (ANC), skilled birth services and postnatal care [102]. Thanks to these interventions, Ethiopia has made notable strides in meeting most of the Millennium Development Goal (MDG) health targets [102]. Promising changes are witnessed in the successive surveys of the Ethiopian Demographic and Health Survey (EDHS) 2001, 2005, 2011 and 2016 in which maternal mortality rate was 871, 673, 676, and 412 per 100,000 live births respectively [75, 125, 127, 128]. The notable accomplishments include achievement of MDG4 with an over two-thirds decline in under-five mortality from the 1990 estimate; this contributed to an increase in average life expectancy at birth from 45 in 1990 to 64 in 2014 [102]. Ethiopia has done remarkably well in improving maternal health with more than $70 \%$ decrease in maternal death in the past 3 decades - in other words, with a reduction of the proportion of mothers dying per 100,000 live births from 1400 in 1990 to 401 in 2017 [99, 102]. An annual maternal death reduction rate of $5 \%$ or more was attained [102]. In addition, the country has shown a significant achievement in halting mortality and morbidity due to malaria, HIV/AIDS, and tuberculosis [102]. 
However, continuity of service and quality of care is not optimal as evidenced by low coverage of skilled delivery, antenatal and postnatal services, and suboptimal uptake of prevention of mother-to-child transmission of HIV services by expectant mothers [102]. Skilled institutional care is decisive in decreasing maternal death [102]. Although there has been improvement compared to the beginning of the new millennium ( $6 \%$ ), only $28 \%$ deliveries were attended by skilled birth attendants in 2016 in Ethiopia [75]. The majority of childbirths in the country $(42 \%)$ were attended by traditional birth attendants [75] .

\subsubsection{Millennium development goals and sustained development goals}

The United Nations Millennium Declaration in the year 2000 established eight international development goals dubbed “The Millennium Development Goals (MDGs)” [129]. The UN member nations agreed to realize these goals by the year 2015 [99, 129].

In conformity with this UN declaration, Ethiopia exerted utmost efforts to achieve the set goals [129-131]. The community focused HEP of Ethiopia played a pivotal role in hastening the country's progress [130] to achieve MDG 4 (reduce child mortality), 5 (improve maternal health) and 6 (combat HIV/AIDS, malaria and other diseases) [129]. A considerable body of literature indicated that HEP accelerated access to primary health care $[102,130]$ and contributed to Ethiopia's success in achieving the health MDGs [131].

The Sustainable Development Goals (SDGs) succeeded the MDGs and came into force on 1 January 2016 [99] for the 15-year period until 2030 [99, 132]. Among the 17 SDGs, the direct health related 13 targets come under SDG 3: "Ensure healthy lives and promote well-being for all at all ages" [99]. The SDG target for reducing the global MMR by 2030 is indicated as SDG target 3.1: "reduce global MMR to less than 70 per 100000 live births by 2030", [96, 99, 132]. In conformity with this, the government of Ethiopia is working hard to achieve SDG 3 to reduce the MMR from 412 deaths per 100,000 live births in 2015 [75] to less than 70 deaths per 100,000 live births by $2030[96,98,131]$.

Ethiopia's flagship community focused programme, HEP, has played a key role in reducing communicable diseases and maternal and under-five mortality $[130,133]$. However, as it is not possible to achieve SDGs by focusing only on communicable diseases [133], and to meet the growing demand for a wide range of quality health services Ethiopia implemented a more comprehensive, resilient and organized second-generation HEP in 2018/19 [133, 134]. The 
second-generation HEP will, therefore, also target on fighting non-communicable disorders that are becoming serious health challenges in the country [133]. The second generation rural HEP aims to upgrade HEWs to level four community health nurses, and renovate, expand, equip and supply health facilities at the grass root level with the essential equipment and supplies [133]. The programme also aspires to shift most essential health services to the community level and to institutionalize and improve the competence of the Women's Development Army Volunteers $[133,134]$.

\subsubsection{Burden of diseases in Ethiopia}

Ethiopia has made major improvements in the health condition of its population in the last two decades [76]. Consecutive EDHS in 2005 [128], 2011 [125] and 2016 [75] have demonstrated declining trends in neonatal, infant, under-five and maternal mortality [76]. Thanks to the government's heavy investment in health $[76,135]$, primary health care service coverage is over 95\% [136, 137], mortality and morbidity due to HIV/AIDS, tuberculosis, and malaria significantly decreased [76] and life expectancy has increased to 66.2 years as of 2018 [76, 116, $135]$.

Despite the progresses mentioned above, the country is still facing the unenviable triple burden of the backlog of communicable diseases, the emerging challenges of non-communicable diseases and injuries that are a challenge to health, national development and social welfare [76, 137, 138]. The large burden of mortality and morbidity is largely attributed to potentially preventable infectious diseases, undernutrition, child and maternal conditions, neonatal diseases, non-communicable diseases and injuries (NCDI) [76, 138, 139]. More than half $(52.1 \%)$ of all deaths in the country are attributable to NCDIs, $43.5 \%$ are due to noncommunicable diseases (NCDs) and $8.6 \%$ are due to injuries particularly road traffic accident, fall and interpersonal violence $[76,116,137]$. On the other hand, $47.9 \%$ of the deaths were due to communicable, maternal, neonatal, and nutritional disorders [116]. There are an estimated 18 road traffic deaths per 100000 population in the country [102]. More than half (54\%) of the NCDIs deaths were due to cardiovascular diseases and cancer [137]. Just over half (51\%) of the NCDI mortalities occur before age 40, and approximately two-thirds (63\%) occurs before age 50 [116]. Mental health disorders and substance-use disorders are among the leading NCD disorders in terms of disease burden [102, 137]. Ethiopia also carries a disproportionately high burden of disability owing to blindness and low vision [102, 137], with over $3 \%$ of the global blindness burden [102]. However, about nine out of ten of the cases of blindness in Ethiopia 
are avoidable [102]. With regard to nutritional problems, national figures indicate that $38 \%$ under-five children are stunted, $10 \%$ of the children are wasted and $24 \%$ of the children are underweight [139]. Available evidence indicates that Ethiopia has a large and diverse burden of NCD risk factors [76] mainly social drug use, low physical activity, raised plasma cholesterol, obesity, hypertension, and high fasting plasma glucose [76, 137].

According to the ministry of health of Ethiopia, there are diverse causes of morbidity, mortality and hospital admission. The top ten causes of morbidity are acute upper respiratory infections (9.6\%), acute febrile illness (9.3\%), pneumonia (7.9\%), diarrheal diseases (7.2\%), dyspepsia $(5.4 \%)$, trauma (injury, fracture etc.) $(4.6 \%)$, urinary tract infection $(4.3 \%)$, infections of the skin and subcutaneous tissue $(4.1 \%)$, helminthiasis (3.6\%), diseases of the musculoskeletal system and connective tissue (3.5\%) [140]. On the other hand, the top 10 causes of admission were single spontaneous delivery (15.5\%), pneumonia (7.7\%), trauma (injury, fracture etc.) $(4.3 \%)$, other delivery $(4.1 \%)$, neonatal sepsis $(2.6 \%)$, other or unspecified obstetric conditions $(2.3 \%)$, other abortion (spontaneous, with complication etc.) (2.2\%), diarrhoea with dehydration (2.1\%), severe acute malnutrition (2.0\%), and non-bloody diarrhoea (1.9\%) [140]. The top ten causes of mortality were prematurity (7.4\%), birth asphyxia (6.3\%), neonatal sepsis (5.8\%), pneumonia (5.6\%), other or unspecified diseases of the circulatory system $(4.3 \%)$, cerebrovascular accident (stroke) (4.2\%), tuberculosis $(3.8 \%)$, other or unspecified perinatal diseases (3.4\%), trauma (injury, fracture etc.) (3.0\%), and AIDS (3.0\%) [140].

\subsection{Rationale of the study}

Women experience different medical conditions during pregnancy, such as nausea, vomiting, morning sickness, fatigue, indigestion/heartburn, common cold, urinary tract infection and swollen ankles [3, 43]. Consequently, the vast majority of women take one or more types of medicinal plants and pharmaceutical medicines (prescription or OTC) during pregnancy [30]. Women also take social drugs for various reasons $[30,78,85]$. However, use of medicinal plants $[3,23,24,141,142]$ and conventional medicines $[6,20,21,30,51]$ also pose a potential risk to the developing foetus and the pregnant woman. Furthermore, due to the diverse active constituents in medicinal plants, conventional medicines and substances, there is risk of interaction among them which will further deteriorate maternal and foetal health [3, 143-145]. Moreover, women take one or more conventional medicine once admitted to hospital and thus there is a possibility for medication related problems (MRPs) that may lead to substantial morbidity and mortality $[68,69]$. Besides, extensive use of medicinal plants $[3,146]$, easy 
access to conventional medicines including antibiotics without prescription [38] and easy availability of medicines outside the formal and authorized organisations coupled with weak regulation and regulatory enforcement in the pharmaceutical sector in Ethiopia $[38,39]$ makes their use during pregnancy more worrisome. Despite this fact, data systematically examining the treatment modalities used and their utilization, prevalence of use, safety profiles, and predictors of use in pregnancy and lactation are still scarce.

Thus far only a few studies have investigated medicinal plant use [3, 19, 147-149] and prescription medicine use or self-medication practice during pregnancy [28, 38, 47, 150-154] in Ethiopia, however, all were conducted among outpatient women, mainly in their early stage of pregnancy, and failed to capture the full picture of use of the various treatment modalities in the entire pregnancy. Likewise, though some MRP identification studies have been conducted among inpatients so far in Ethiopia [155-159], all have focused on the non-pregnant patient population. Thus far, no study has investigated MRPs in pregnant and lactating inpatients in a hospital setting in Ethiopia. Furthermore, the majority of studies of pharmaceutical medicine and medicinal plant use were conducted almost a decade ago, and were not from southwest Ethiopia. Moreover, these studies were of small sample size. Since smaller size studies have diminished statistical power and representativeness, they do not normally yield reliable or precise estimates and lead to less conclusive results. Similarly, few studies have assessed the use of one or more social drugs among women in communities or antenatal care (ANC) settings in Ethiopia and almost all of them were carried out in other corners of Ethiopia [78, 91, 160162]. Only limited studies have been conducted among ambulatory patients at healthcare facilities in southwest Ethiopia [87, 163], and they are of smaller sample size and focused only on the use of khat during pregnancy.

Thus far, no study has investigated the use of medicinal plants, conventional medicines, or substances, and treatment related problems, medicine safety concerns and the associated contributing factors in an obstetrics group in an inpatient hospital setting in Ethiopia. Therefore, generating knowledge about the use of medicinal plants and medicines during pregnancy based on a relatively large sample size will be an important input for evidence based maternal healthcare and policy design. The results from this study could contribute to the formulation and implementation of risk reduction strategies during pregnancy, which in turn will help in achieving the SDG maternal health goals. Therefore, the overall aim of this project is to generate knowledge about the use of medicinal plants and conventional medicines and their safety 
profiles in pregnancy. The study also aimed to investigate MRPs encountered among pregnant and lactating women in the maternity and gynaecology wards of Jimma University Medical Center (JUMC) in Southwest Ethiopia. 


\section{AIMS OF THE DISSERTATION}

The overall objective of this work was to increase knowledge about the prevalence, utilization, and safety of medicinal plants and pharmaceutical medicines used among pregnant and lactating women, through a series of studies that addressed the following specific objectives:

\section{Study I:}

- To summarize the existing evidence on the prevalence and diversity of medicinal plants use by women during pregnancy in Africa

- To summarize the existing evidence on the types, utilization patterns, reasons for use, and safety of the different medicinal plant species used by women during pregnancy in Africa

\section{Study II:}

- To determine the prevalence, predictors and the types of medicinal plants used among pregnant and lactating women at JUMC, Southwest Ethiopia

- To assess safety profiles, utilization and sources of the medicinal plants used among pregnant and lactating women at JUMC, Southwest Ethiopia

\section{Study III:}

- To determine the prevalence and predictors of self-medication during pregnancy and lactation at JUMC, Southwest Ethiopia

- To evaluate the pregnancy safety profiles of conventional medicines used during pregnancy and lactation at JUMC, Southwest Ethiopia

- To assess the prevalence and types of social drugs used among pregnant and lactating women at JUMC, Southwest Ethiopia

\section{Study IV:}

- To investigate the prevalence, clinical significance, and risk factors of medicationrelated problems (MRPs) among pregnant and lactating women at JUMC, Southwest Ethiopia 


\section{SUBJECTS AND METHODS}

\subsection{Study design and period}

This dissertation is based on a systematic review study (described here as the systematic review), and a hospital based cross-sectional study design (described here after as the hospital based study). In the first part of the project (Paper I), a systematic literature search was conducted from July 2016 to January 2017. In the hospital based study part of the project (Papers II-IV), hospital-based cross-sectional study was used to assess medicinal plant use and safety profiles of the medicinal plants; the prevalence, clinical significance, and risk factors of MRPs, and self-medication practice and pregnancy safety profiles of medicines used among pregnant and lactating women at JUMC in Southwest Ethiopia from February to June 2017.

\subsection{The systematic review}

\subsubsection{Literature search strategy}

We searched for literature that documented the prevalence of use of medicinal plants related to pregnancy in Africa. We undertook a systematic literature search including published scientific literature, conference proceedings, unpublished studies, theses and dissertations. Medline, Embase, African Journals OnLine (AJOL), Google Scholar and Biological Abstracts bibliographic databases were searched for articles on medicinal plants use during gestation. Moreover, Google search engine and various African or international universities' websites were screened for more studies about the use of medicinal plants during pregnancy. To ensure literature saturation, additional studies were also identified through scanning the reference lists and related articles of included studies and by contacting experts. All available original research works on medicinal plants from the African continent which were made available during the research period were reviewed.

In the literature search process, the names of the databases searched, the keywords used and the search results were retained as a 'search diary'. Titles and abstracts of studies considered for retrieval were saved to a Mendeley reference manager with duplicated items removed, along with details of where the reference had been found. In addition, inclusion/exclusion decisions were documented in the Mendeley reference manager, and in accordance with this, the retrieved studies were filed. 


\subsubsection{Study selection and data extraction}

Studies were included if they described the prevalence of medicinal plant use among expectant patients in Africa. The search was not restricted to a specific language, time of publication or publication status.

Two independent reviewers selected papers according to the pre-defined inclusion criteria. First, the reviewers independently inspected the title of every record retrieved considering the inclusion criteria. Next, abstracts were appraised to match the selection criteria. Third, all abstracts that potentially appeared suitable for the review as well as those that could not give sufficient information to make an informed judgment, were retrieved and assessed as full text. Finally, three reviewers extracted information from qualifying papers according to a predefined data extraction spreadsheet. Data were summarized in narrative form, and compared by the reviewers. Differences in opinion on extracted data were resolved by referring to the original study until consensus was reached.

\subsubsection{Studies included in the systematic review}

For the systematic review, a total of 3659 medicinal plant use studies were retrieved, but only 303 studies received full-text assessment for eligibility and, finally, only 50 papers fulfilled the selection criteria for review and data extraction.

\subsection{The hospital based study}

\subsubsection{Study setting}

The hospital based study was conducted at Jimma University Medical Center (JUMC), a public tertiary teaching medical center located in the city of Jimma, $350 \mathrm{~km}$ Southwest of Addis Ababa. Currently it is the only teaching and tertiary level referral hospital in south-west Ethiopia with over 800 bed capacity and a predominantly rural catchment population of around 20 million people $[164,165]$. The Center provides service for approximately 160,000 outpatients and 45,000 in-patients annually [166]. The hospital delivers healthcare in areas like gynaecology and obstetrics, surgery, paediatrics and child health, internal medicine, ophthalmology, psychiatry, dermatology, anaesthesiology and dentistry [165]. It has out-patient and in-patient services, maternal and child health services, referral and follow-up services, physiotherapy and rehabilitative services, intensive care and recovery services [167]. JUMC also serves as a clinical postgraduate specialty teaching hospital for different specialties in many 
of its departments, including obstetrics and gynaecology [165]. The JUMC obstetrics and gynaecology department has a bed capacity of 265 and provides specialized health services for about 7,600 inpatients, and 11,600 outpatients every year.

The department of obstetrics and gynaecology has two wards (gynaecology and maternity/labour), one ANC outpatient clinic, one general gynaecologic outpatient clinic, one family planning clinic and a referral clinic where cases with gynaecology oncology, benign gynaecologic diseases, and high-risk pregnancy in which patients are examined on different days of the week [165]. Obstetrics and gynaecology department has 8 senior obstetricians and gynaecologists and 43 residents of different years (levels) of study [165]. The inpatient ward provides medical care for maternity and gynaecology patients (mostly delivery services and management of pregnancy-related complications), whereas outpatients are mainly healthy child bearing women who visit the ANC for their regular maternity check-ups. As per the hospitals classification, those pregnant patients who are below 28 weeks of gestation are admitted and treated at the gynaecology inpatient ward. Most parturient women admitted in the gynaecology ward are elective or spontaneous abortions, hyperemesis gravidarum or others with early gestation health concerns. Women who are at 28 weeks of gestation and above are admitted and treated at the maternity inpatient ward. Women having a vaginal delivery give birth in the labour ward, and are then admitted to the maternity ward. If the mother and newborn are healthy, they are discharged at the earliest possible time after delivery. Women having a caesarean delivery are transferred to the obstetrics ward and usually stay for three days. 


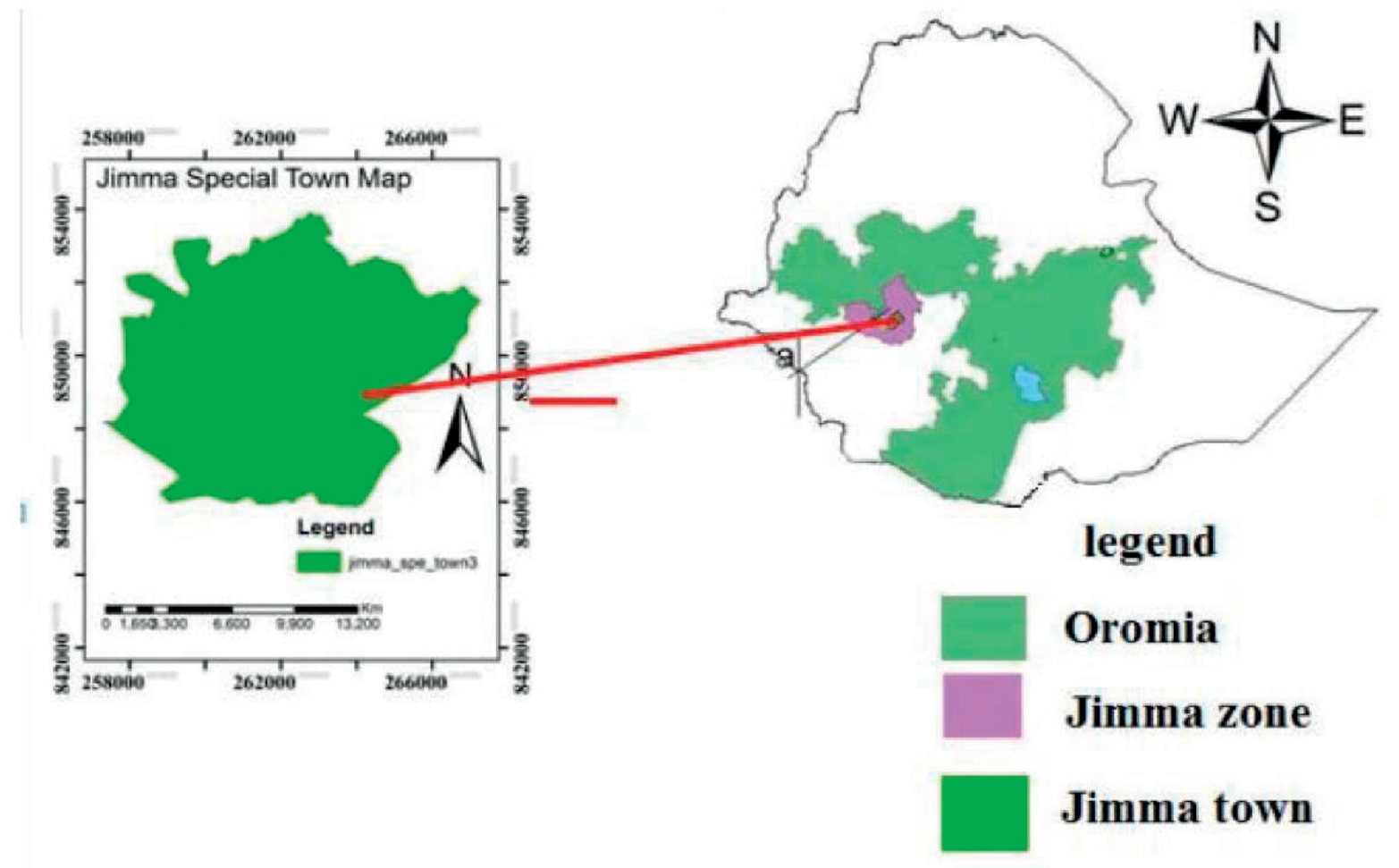

Figure 2: The town Jimma where Jimma University Medical Center is located

[Source: Sorsa, A.; Senadheera, S.; Birru, Y. Engineering Characterization of Subgrade Soils of Jimma Town, Ethiopia, for Roadway Design. Geosciences 2020, 10, 94]

\subsubsection{Source population and study population}

\subsubsection{Source population}

The source population includes all pregnant or postpartum patients who were admitted at the maternity/labour and gynaecology wards.

\subsubsection{Study population}

The study participants were pregnant or postpartum patients who were admitted to the study wards during the data collection period and who gave written informed consent.

\subsubsection{Inclusion and exclusion criteria}

Patients were eligible if they were admitted at the maternity/labour and gynaecology wards at the time of data collection and willing to participate in the study. Patients were excluded from the study if they were under 18 years of age, critically ill, not willing to participate, with hearing difficulty, communication difficulties, inablility to speak or with mental illness, admitted for a brief time (less than four hours), and non-pregnant women admitted in the gynaecology ward. 


\subsubsection{Study variables}

\subsubsection{Dependent variables}

- Medicinal plant use

- Medication-related problem

- Self-medication practice

- Safety class of medicine

- Social drugs use

\subsubsection{Independent variables}

Patient-related (Socio-demographic) variables: age, level of education, marital status, occupation, religious affiliation, ethnic group, family size, place of residence, alcohol use, $k$ hat chewing, tobacco smoking.

Disease or pregnancy ailment-related variables: patient admission ward (Gynaecology ward and Maternity ward), past medical history, chronic disease, length of hospital stay, gestation-related illness and related symptoms.

Pregnancy/obstetric-related variables: parity, gravidity, gestational age, adverse pregnancy outcome (current and previous), obstetrics category (caesarean and vaginal delivery).

Medicine-related variables: past medication (prescription and non-prescription medicine use), medicines used during admission and discharge, ferrous sulphate supplementation, number of medicines used, adverse drug reaction history.

Health professional /facility-related variables: walking distance to the nearest health facility, availability of preferred medication for a specific condition, pharmacist intervention. Medicinal plant-related variables: type, number and safety profiles of medicinal plant used, reason for use, utilization, information sources.

\subsubsection{Sample size}

The sample size was determined using the Kish single population proportion formula [168] with the assumptions of $80 \%$ power, at $95 \%$ confidence level, $3 \%$ error margin, and $5 \%$ type I error. Due to lack of any published study that determined the prevalence of medicinal plant use, self-medication, or medication-related problems (MRPs) occurring among hospitalized expectant mothers in Ethiopia, the proportion of any of the conditions being studied was estimated as 50\%. This makes the required sample size 1,067 pregnant women, which was then further increased to 1,121 to account for a predicted $5 \%$ non-response rate. 


\subsubsection{Data collection and procedures}

A pre-tested data extraction form and a face-to-face interviewer-administered structured questionnaire were used for data collection. Consecutive patients who fulfilled the inclusion criteria were enrolled in the study from JUMC maternity/labour and gynaecology wards from February to June 2017.

Five clinical pharmacists (MRP assessment and data abstraction part) and four nurses (the questionnaire part) from JUMC collected the data. Data collectors were given two days of training before commencing data collection. Training was given by the principal investigator on the objective of the study, methods of data collection including data extraction from patient medical records/patient charts, assessment of the presence and development of MRPs, techniques of interviewing patients, verify the completeness of the filled questionnaire and data abstraction foms. Data were collected at the appropriate and convenient time for them. The $\mathrm{PhD}$ student supervised the data collection and rechecked the completeness of each questionnaire and data abstraction form every day.

Data collectors followed each patient included in the study daily from the time of admission until discharge to assess presence and development of MRPs. Specially designed data extraction forms were used for data collection. Data were abstracted by reviewing patients' medical cards, patient medication charts (Medication regimen administration) and laboratory findings and by interviewing patients about diagnosis, management and follow up. Data collectors also interviewed patients on admission about their past medical and medication history.

\subsubsection{Development of data collection tools: questionnaire and data abstraction form}

The data collection questionnaire was developed based on a review of relevant literature [19, 147, 169-172]. The bilingual questionnaire was initially developed in English, then translated into the local languages, Amharic and Afan Oromo, to suit the target population and then backtranslated into English by different persons to ensure semantic equivalence. The translation and back translation of the study questionnaire was performed by lecturers from Jimma University fluent in English and their own local language with previous experience of translating questionnaires. Similar to the questionnaire, the data extraction form was developed based on a review of relevant literatures [59, 60, 62, 68, 69, 157]. Both the questionnaire and the data extraction form were validated by piloting and through expert review. The tool was pre-tested on a sample of 30 inpatient pregnant or post-partum women at Shenen Ghibe district hospital 
located in Jimma city, to assess content validity, content consistency, comprehension and possible defective questions and the time needed to complete it. Based on the pre-test and feedback from researchers experienced in developing study questionnaires, the final bilingual questionnaire was amended accordingly and data collectors received clarification on items which were not understood well. The English version of the data extraction form was used and appropriate changes were made based on expert opinion and feedback received from the pilot study.

\subsubsection{MRP identification, assessment and clinical significance}

\subsubsection{MRP identification and assessment}

A panel of experts comprised of senior clinical pharmacists and obstetricians/gynaecologists was formed to assess and identify MRPs. The panel of experts identified and classified MRPs categories as recommended by Cipolle et al. [60]. The panel further refined MRP identification and classification method to the study setting in accordance with Ethiopian standard treatment guidelines and literature reviews [58, 68, 173-175]

The data collector clinical pharmacists independently assessed, identified and categorized potential MRPs by reviewing patients' medical cards and medication charts, assessing laboratory investigations and interviewing patients about medication experience. The probability of drug interaction was evaluated using Medscape, WebMD and Epocrates drug interaction programmes. Specific information about medication therapies, such as the recommended medication of choice, recommended dosages, frequency of administration, duration of therapy and safety were compared with reference to national and international treatment guidelines and standard text books $[58,68,173-175]$. Then, they recorded the MRPs on MRP registration forms and discussed the findings and possible resolutions with the healthcare team every day. If any MRPs were considered to require urgent action, possible intervention measures were proposed and communicated to the healthcare professionals to resolve or prevent them on the spot. On the other hand, less-urgent MRPs were documented along with recommendations on a separate report as they were identified and validated by the panel of experts during meetings on a daily basis. The collected data were analysed and interpreted for the assessment of MRPs using standard criteria. The panel reached consensus for any discrepancy by discussion and they categorized MRPs when necessary. Finally, only MRPs on which the panel had consensus were included in this study. For problems related to patient's knowledge about the medication, the pharmacist discussed the problem with the 
patient and gave recommendations. At discharge, the pharmacist provided the patients with information on the safe administration of their medicines. The data collectors recorded the outcomes of the discussions with health-care team on a data collection form as either accepted (when the health-care team accepted the recommendations), or not accepted (when the healthcare team did not agree with the recommendations). When an MRP was identified, the possible intervention measures were proposed and the prescribers were communicated to resolve or prevent the MRP for ethical reasons. Finally, the identified MRPs were classified by evaluating four distinct criteria of pharmacotherapy: indication, effectiveness, safety, and adherence.

\subsubsection{Clinical significance of MRPs}

Clinical significance classification of MRPs was done based on the Thompson et al. [68] modified and simplified 2-level severity scale, with clinical significance categorised as either low (level 1) or moderate to severe (level 2) potential to result in patient deterioration or discomfort. The clinical significance, similar to MRP assessment, was initially evaluated by clinical pharmacists assessing the MRP and was subsequently discussed and reviewed by the panel of experts. The description of the MRPs, their clinical significance, and medication(s) involved were recorded using a standardized form.

Medications involved in MRPs were classified according to the World Health Organization (WHO) first level of the Anatomical Therapeutic Chemical classification system (ATC) that categorises medications into 14 main groups [176].

\subsubsection{Safety Classification}

\subsubsection{Safety classification of medicinal plants}

Medicinal plants were classified into four safety categories: safe, caution, contraindicated or unknown for use during pregnancy based on previous studies [23, 24]. Several reference sources were reviewed in order to capture diverse perspectives when classifying medicinal plants. Reference text books [177-179], literature reviews [3, 23, 24], monographs and Ovid MEDLINE and Ovid EMBASE bibliographic databases were used to evaluate safety of the medicinal plants in gestation. Data from animal studies were used if human studies were absent. If a medicinal plant preparation was composed of two or more plants, each plant was individually evaluated and classified. 


\subsubsection{Safety classification of conventional medicines}

The two internationally acknowledged risk classification systems, the U.S. Food and Drug Administration (US FDA) [55] and the Australian Therapeutic Goods Administration (AUTGA) [56], were used to indicate the potential of a medicine used for self medication to cause foetal injury if used in pregnancy. The FDA classified drugs into five letter risk categories, A, $\mathrm{B}, \mathrm{C}, \mathrm{D}$, and $\mathrm{X}[55]$, from those with the least risk (category A) to those that are highly toxic and cause severe birth defects (category X). In 2015, the FDA replaced the former pregnancy risk letter classes with narrative risk summary based on available data and the letter category system no longer used [55]. The FDA classification system was used as the primary categorization method in this study because it is still widely in use in Ethiopia [28, 38]. However, if the FDA classification fell short, the AU-TGA classification system [56] was used. The Australian system has classes (A, B1, B2, B3, C, D and X) for the classification of foetal risk of medicines. In order to facilitate the safety analysis and to make groups of more clinical interest, medicine exposures were classified into "probably safe"'(the FDA categories A and $\mathrm{B}$, and the AU-TGA categories A, B1, and B2), "potentially risky" (FDA Categories C, D, and $\mathrm{X}$ and AU-TGA categories B3, C, D and X) or "unclassified"'(medicines that could not be classified by either of the systems) based on similar studies [44, 48]. Ethiopian epidemiological profile, national formulary and standard treatment guidelines and the WHO recommendations were referred to modify findings from the FDA or AU-TGA groupings when need arises [58, $174,175,180]$. For combination products the categorisation was based on the component with the most restrictive category.

\subsubsection{Data management and analysis}

In Paper I, three of the authors appraised the articles selected for systematic review. Data were extracted into spreadsheets according to pre-defined criteria and were summarized in narrative form. The reviewers compared the summarized data and any differences of opinion were resolved by discussion and consultation with the original study. Any differences of opinion were resolved in consultation with the third author. In the hospital based study, the data were checked for completeness, coded, entered, cleaned and analysed using Statistical Package for the Social Sciences (SPSS) software version 25.0 for Windows $\left(\mathrm{IBM}^{\circledR}\right.$ SPSS $^{\circledR}$ Statistics, IBM Corp, Armonk, NY, USA). Descriptive statistics in the form of frequencies (proportions) and percentage for categorical data and means with standard deviations and medians with ranges for continuous variables were used to summarize outcomes and explanatory variables. Descriptive analysis was also conducted to assess inconsistencies, outliers, and missing values. 
The dependent and independent variables were entered into a bivariate logistic regression analysis, one by one, in order to estimate the strength of association using Odds Ratios (OR) with 95\% confidence intervals (CIs). Then all explanatory variables associated with the outcome variable (with $\mathrm{P}<0.05$ in Paper II and Paper IV and $\mathrm{P}<0.25$ in Paper III) were entered together into a multivariate logistic regression. Statistical significance was defined as a 2-sided $\mathrm{P}$ value of $<0.05$. In the case of Paper IV, as iron was involved in almost half $165(41.9 \%)$ of the MRPs, a post hoc logistic regression analysis was done excluding iron.

\subsubsection{Ethics and approvals}

The systematic review study did not require ethical approval from any research ethics committee as the information analysed was obtained from already conducted studies. Jimma University Institute of health Institutional Review Board (IRB) in Ethiopia and The Regional Committees for Medical and Health Research Ethics (REK) in Norway approved the hospital based study. In addition, a formal letter of cooperation from Institute of Health IRB was given to JUMC. The study was commenced after letter of support was written to the department of obstetrics and gynaecology from JUMC to facilitate the study. Pregnant or post-partum women were clearly informed about the purpose, benefit, procedures and content of the study. Women were also clearly informed about the voluntary nature of the participation, the right not to participate or withdraw their consent at any time without any effect on their medical care. Then written informed consent was sought from each woman who agreed to participate in the study before the data collection. Anonymity and confidentiality were maintained through removing woman's name and any other identifiers, and restricting data access to the research team members only, respectively. 


\section{RESULTS}

\subsection{The use of medicinal plants by pregnant women in Africa: A systematic review}

\section{(Paper I)}

In order to achieve an overview of all the available evidence about medicinal plants use by pregnant women in Ethiopia and in Africa at large, and to identify knowledge gaps that call for more research, a systematic review was done at the start of the $\mathrm{PhD}$ project.

The aim of Paper I was to summarize the prevalence and diversity of medicinal plants used by women in pregnancy in Africa. Furthermore, Paper I aimed to analyse the prevalence, types, reasons for use, possible health risks and utilization patterns of medicinal plant species.

The search identified 50 research papers on the use of medicinal plants for inclusion in the review. The most common region of research was West Africa (28 studies); Nigeria alone generated 21 studies. Only 5 studies were found from Ethiopia and none of them was from the southwest area. The prevalence of use of medicinal plants during gestation varied widely within and across African regions and countries (2\% to 100\%). In total, expectant mothers used 274 plant species belonging to 87 plant families to treat various illnesses. The most frequently used plant species were Zingiber officinale Roscoe (15 studies), Allium sativum L. (12 studies) and Cucurbita pepo L. (7 studies). Except ginger (Z. officinale), almost all of the medicinal plants are understudied and dependable data are scarce regarding their safety, effectiveness, and effect of long-term use.

The most common reasons for use of botanical medicines were alleviation of gestationassociated symptoms $(26 \%)$ and stimulation or facilitation of labour and delivery $(14 \%)$. Concomitant use of medicinal plants and conventional medicines were reported in 10 studies (20\%), with prevalence ranging from $2.4 \%$ to $77.3 \%$. Various plant parts, preparation methods and administration routes were used. Notably, 38 (76\%) studies indicated that botanical medicines were most frequently administered by oral route.

Many factors were significantly associated with medicinal plant use $(\mathrm{P} \leq 0.05)$ including lower educational level, older age, being married, poor pregnancy outcome, previous medicinal plant use experience, perception that medicinal plants are effective, large family size, and rural residence. 


\subsection{Medicinal plants used among pregnant women in a tertiary teaching hospital in Jimma, Ethiopia: a cross-sectional study (Paper II)}

The aim of Paper II was to determine the prevalence, predictors and the types of medicinal plants used among pregnant women at the maternity and gynaecology ward at JUMC, Southwest Ethiopia. Moreover, Paper II aimed to assess utilization, sources, and safety concerns of medicinal plants used in gestation.

Overall, $319(28.6 \%)$ of the expectant mothers reported use of at least one medicinal plant in gestation, with an average of 1.5 medicinal plants per woman (range 1 to 8). Twenty-seven types of medicinal plants were used and the most commonly used was L. usitatissimum (flaxseed) (77.1\%). Depending on available information in the current literature, only five of the 27 medicinal plants used by women were classified as safe to use in pregnancy.

The most common reasons for use of medicinal plants were to induce or to reduce the intensity and shorten duration of labour (60.2\%), common cold/flu (20.4\%) and preparation of labour (15.7\%). Most of the medicinal plants were used during labour (32.2\%) followed by third trimester $(27.2 \%)$. A substantial proportion of women purchased medicinal plants at market places $(76.5 \%)$ and/or got it through family members $(68.3 \%)$. Slightly over three fourths of parturient women were recommended by their family members $(75.2 \%)$ to use medicinal plants. With reference to the utilization pattern, use of seeds (57.6\%), dry plant materials $(60.1 \%)$ and oral route of administration (89.7\%) were predominant. Furthermore, water glass dosage measurement unit (51.7\%), one water glass dose (47.5\%), once per day frequency (54.8\%), and almost use in entire gestational duration (32.9\%) and one episode of medicinal plant use $(46.0 \%)$ in pregnancy were most commonly reported.

Several maternal and sociodemographic characteristics were significantly associated with the use of medicinal plants in gestation $(\mathrm{P}<0.05$; all are at 95\% CI) including scarcity of health facility (adjusted OR 6.92; 1.77, 27.10), admission in the maternity wards (adjusted OR 2.80; 1.43, 5.48), khat chewing (adjusted OR 2.53; 1.46, 4.39), alcohol drinking (adjusted OR 2.43; $1.28,4.62$ ), modern medicine use (adjusted OR 1.83; 1.36, 2.46), chronic disorders (adjusted OR 1.83; 1.04, 3.24), and secondary school educational level (adjusted OR 1.54; 1.01, 2.36) were significantly associated with the use of medicinal plants in gestation $(\mathrm{P}<0.05)$. 


\subsection{Self-Medication and safety profile of medicines used among pregnant women in a tertiary teaching hospital in Jimma, Ethiopia (Paper III)}

The aim of Paper III was to determine the prevalence and predictors of self-medication in pregnancy at JUMC in Ethiopia. Paper III also evaluated pregnancy safety profiles of medicines used based on risk classification techniques developed in the U.S. and Australia. Moreover, this study assessed the prevalence and types of social drugs used among the expectant mothers.

Of the 1117 expectant mothers, $300(27.0 \%)$ had practiced self-medication with modern medicines. Analgesics (92.3\%) were the most frequently used medicine groups mainly paracetamol (72.7\%) and diclofenac (11.0\%). Seven percent of the parturient mothers selftreated with antimicrobials, mainly amoxicillin (5.7\%). Nearly three out of four women $(73.4 \%)$ self-cared with medicines classified as probably safe. Potentially risky medicines were used by $45(13.6 \%)$ women mainly diclofenac 33 (10.0\%). Almost four out of ten $(36.7 \%)$ of the selfmedicated gestating women had concurrently consumed medicinal plants.

The study found that women who profess Islam (adjusted OR 2.22; 1.19, 4.17), or Christian Orthodox religion (adjusted OR 2.04; 1.06, 3.92) and used medicinal plant in the current gestation (adjusted OR 1.78; 1.33, 2.40) were more likely to practice self-medication than their respective counterparts $(\mathrm{P}<0.05)$. On the contrary, women who had access to a health facility in a nearby residential area (adjusted OR $0.62 ; 0.41,0.95$ ) were less likely to self-medicate during pregnancy $(\mathrm{P}<0.05$; all are at $95 \% \mathrm{CI})$.

A total of 108 (9.7\%) pregnant women used at least one type of substance. Almost five percent of the gravid women used alcohol, of which $67.4 \%$ of them daily drank variable amounts of alcohol. Over $5 \%$ of the expectant mothers chewed khat almost daily. None of the pregnant women was an active tobacco smoker.

Orthodox Christian (adjusted OR 4.08; 1.19, 13.99) or Islam religion (adjusted OR 3.79; 1.13, 12.76), admission to the gynaecology ward (adjusted OR $2.81 ; 1.31,6.04$ ), and medicinal plants use during pregnancy (adjusted OR 2.75; 1.79, 4.24) were significant predictors of social drug use. On the contrary, shorter hospital stay (adjusted OR 0.63; 0.41, 0.95) was inversely associated with social drug use $(\mathrm{P}<0.05$; all are at $95 \% \mathrm{CI})$. 


\subsection{Medication-related problems among hospitalized pregnant women in a tertiary teaching hospital in Ethiopia: A prospective observational study (Paper IV)}

The aim of Paper IV was to investigate the frequency and nature of medication-related problems (MRPs) among hospitalized pregnant women at the maternity and gynaecology wards at JUMC, Southwest Ethiopia.

Most of the study participants $992(88.8 \%)$ were admitted in the maternity ward and the remaining $125(11.2 \%)$ in the gynaecology ward. Hospitalized gravid or breastfeeding women were prescribed with up to 24 medicines (median: 3 medications per patient, range: 0-24 medications) and approximately half $(42.7 \%)$ of the women were on polypharmacy $(\geq 5$ medications). Pitocin (63.7\%), normal saline (38.9\%), and ceftriaxone (36.0\%) were the most commonly administered medicines in in-patients. Ferrous sulphate (54.4\%), cephalexin (30.4\%), and metronidazole (25.0\%) were the top three prescribed discharge medications.

At least one MRP occurred in 323 (28.9\%) hospitalized pregnant and lactating women (totally 394 discrete MRPs). The majority of MRPs, $87.6 \%$, were identified in the maternity ward. The most common therapeutic groups implicated in MRPs were medications acting on blood and blood-forming organs, mainly ferrous sulphate (35.3\%), followed by anti-infectives for systemic use, largely cephalexin and metronidazole (each 9.4\%). Need for additional drug therapy 236 cases (73.1\%), need for an additional laboratory test 41 cases (12.7\%), unnecessary drug therapy 38 cases $(11.8 \%)$, and too low dosage 38 cases $(11.8 \%)$ were the frequently encountered MRPs categories. Concerning severity, 278 (70.6\%) of the MRPs were classified as level 2 MRPs, of which 133 (47.8\%) resulted from iron treatment/supplementation.

Compared to primaparous or younger women, nulliparous, multiparous and more than 26- yearold women were significantly more likely to experience MRPs (1.63 - 1.82 fold increased likelihood). Because iron was involved in almost half 165 (41.9\%) of the MRPs, a post hoc logistic regression analysis was performed without iron. In the post hoc analysis compared to their respective counterparts, only one medication (adjusted OR 2.38; 1.24, 4.56) or two or more medications use prior to admission (adjusted OR 2.21; 1.12, 4.38), chronic disease (adjusted OR 1.91; 1.02, 3.58), nulliparity (adjusted OR 1.99; 1.22, 3.24) and multiparity (adjusted OR 1.91; 1.17, 3.12) were significantly associated with increased likelihood of encountering MRPs $(\mathrm{P}<0.05$; all are at 95\% CI). 


\section{DISCUSSION}

\subsection{Summary of findings}

This dissertation aimed to understand medicinal plants and pharmaceutical medicines use in pregnancy in Ethiopia with a focus on prevalence, utilization pattern and safety. The details of the research project findings are described and discussed in Papers I-IV and in the discussion below, only the summary of the most relevant results are presented and discussed. However, it is difficult to compare the results of this work with previous studies due to paucity of similar research and differences in data gathering methods and study objectives.

The study found that the prevalence of medicinal plant use during pregnancy in Africa ranged from $2 \%$ to $100 \%$, and that the prevalence of simultaneous use of medicinal plants and conventional medicines during pregnancy ranged from $2.4 \%$ to $77.3 \%$. The hospital-based study showed that $28.6 \%$ women used medicinal plants and $27.0 \%$ self-medicated with conventional medicine during pregnancy. In this study, among the 1117 women admitted to the maternity and gynaecology wards, $24.3 \%$ women had concomitantly used medicinal plants and conventional medicines prior to hospital admission. Of those women who used medicinal plants during pregnancy, $16 \%$ had concomitantly used one or more social drug. Among women who self-medicated with conventional medicines, $36.7 \%$ had concomitantly used medicinal plants, and $10.7 \%$ had concomitantly used social drugs. The prevalence of MRPs in the study hospital was found to be $28.9 \%$ mainly in connection with lack of iron supplementation. Various maternal and sociodemographic factors were significantly associated with medicinal plants and conventional medicines use and experiencing MRPs.

In this dissertation, the prevalence of use of the various treatment modalities are discussed first, followed by reasons for use. Third, utilization pattern is discussed. Fourth, the dissertation discusses the safety concerns of treatment modalities. Next, factors associated with use of treatment modalities and MRPs are discussed. Finally, this section discusses the methodological considerations followed by the strengths and limitations of the study.

\subsection{Discussion of the main results}

\subsubsection{Prevalence of use of medicinal plants and pharmaceutical medicines}

To the best of my knowledge no previous study has examined medicine use, including both medicinal plants and modern medicines during pregnancy in an in-patient hospital setting using 
such a large sample size in Ethiopia, particularly in southwest part of the country. The study found that women widely use various types of treatments during gestation, hospital stay and discharge.

Ethiopia is expanding health facilities [that provide western healthcare] in both urban and rural areas to achieve universal health coverage $[76,102,181]$. The country has implemented its innovative community-based flagship Health Extension Programme (HEP) and has built a health post and assigned two female health extension workers at every kebele (a cluster of villages) level to provide healthcare at the grass root level [102]. Nevertheless, there are many barriers that influence maternal health services including scarcity of health facilities and health healthcare professionals that provide delivery service, poor awareness about danger signs and benefits of obstetrics care, lack of proper infrastructure, lack of privacy, affordability, workload, lack of logistic supplies and equipment, and scarcity of pharmaceuticals [93, 181-184]. Fear of mistreatment by health workers, trust in traditional birth attendants and cultural and beliefs that restrict a woman's use of health facilities are additional barriers to accessing maternal health services $[93,181]$. Due to all these reasons, the majority of expectant mothers, especially in rural areas, in Africa in general and in Ethiopia in particular, rely on medicinal plants for primary healthcare $[3,185]$. In spite of this, the use of and healing potentials, safety and efficacy of indigenous herbal medicines have not been scientifically examined [3, 8, 185].

Medicinal plant use among pregnant Ethiopian women has been investigated by some studies (Paper I). However, their sample sizes have been very limited and were neither from southwest Ethiopia nor among inpatients. Moreover, none of the studies analysed safety and detailed utilization. Because all previous studies focused on outpatients, particularly botanical medicines used in late stages of pregnancy were not assessed (Paper I). The prevalence of medicinal plant use among women carrying a child in the study area was $28.6 \%$ (Paper II) which lies within the range reported in Africa in general (2\% to $100 \%)$ and in Ethiopia in particular (2.0\% to $73.1 \%$ ) (Paper I). Compared to research findings across Africa, the overall rate of use of medicinal plants reported in this study is lower than the average prevalence rate of medicinal plant use reported from East Africa region (32\%) (Paper I), for which there are several possible explanations.

The variation can be attributed to the study settings, recall periods, study participants, and health facility accessibility. Unlike studies in other areas, this study did not consider herbal meal 
preparations and nutritional supplements as medicinal plants (Paper I) and this could have resulted in a lower rate of use. In Ethiopia, the official health care system is almost entirely based on the concept of modern medicine and the use of medicinal plants or other indigenous forms of remedy is considered harmful or only used by the poor and not supported by modern healthcare practitioners. Therefore, pregnant women admitted to hospital are usually more reluctant to speak to researchers about their use of medicinal plants because they fear that the investigators might inform the hospital healthcare professionals about their practices who would, in turn, reproach, victimize, discourage and possibly stop them from using medicinal plants. Thus, it is possible in this study that some women who had used plants in pregnancy may have deliberately avoided disclosing it. Moreover, literature shows that lack of access to maternal healthcare facilities is the foremost factor for resorting to traditional medicine in Africa (Paper I). Nevertheless, primary healthcare facilities were relatively available and accessible to many women in this study (Paper II), and they might have opted to mainstream maternity care resulting in a lower rate of medicinal plant use. To better use indigenous knowledge and improve pregnancy outcomes, pregnant women should be advised about the concerns, and encouraged to report their medicinal plant use history during health facility visit. Healthcare professionals also should ask women about such use.

The prevalence of self-medication with conventional medicines $(27.0 \%)$ in this study lies within the range of $1.9 \%$ to $29.1 \%$ reported in Ethiopia [28, 47], and elsewhere, $2.2 \%$ to $72.4 \%$ [36, 37, 41]. Differences in recall period in which women were asked to remember over a 9-month period and study setting in which hospitalized women were our study participants could have contributed for the observed variation. In addition, variation in methodology, disease epidemiology, culture among participants and settings may have contributed to the disparity. Differences in restriction policies on dispensing medicines, over-the-counter medication patterns and medication prescription systems in different countries may partly contribute to the discrepancy in the stated rate of self-medication. Differences in drug regulatory enforcement and informal market control are additional contributors for the observed disparities.

In general, the results of this study indicate that despite its risks in pregnancy, self-medication is common in Ethiopia, similar to other corners of the globe. However, only $42 \%$ of Ethiopian women are literate [75] and the pharmaceutical labels are not in local languages. Moreover, the majority of women lack specialized understanding of the principles of pharmacotherapy and are unable to comprehend pharmaceutical prescription labels and pharmaceutical product 
information sheets. Therefore, such arbitrary use without physician consultation may increase the risk of inappropriate use and endanger maternal and foetal health. Thus, awareness creation on rational drug use and adherence to prescription and dispensing guidelines are vital for safe use of medicines.

\subsubsection{Reasons for use of medicinal plants and conventional medicines}

Unlike previous findings from Ethiopia and other parts of Africa in which women reported NVP as their primary reason for using medicinal plants (Paper I), this study found that the primary reason for use of medicinal plant was facilitation of child birth (preparation for labour, induction, reduction, or shortening of labour) (Paper II). The common indications of plant remedies were for cold/flu and nausea in Europe [142], nausea and constipation in North America [142] and, gastrointestinal disorders management (nausea, vomiting, bloating, and stomach aches) in the Middle East [186]. The difference in reasons for use from other corners of Ethiopia and regions elsewhere may be due to difference in climate, geographical location (which will affect the types of plants that commonly grow in that area), accessibility and disease pattern. Besides, unlike previous studies, the participants in this study were hospitalized women expecting their baby, and who most probably only report the recent reasons for use of medicinal plants, i.e., facilitation of child birth. In addition, methodological approaches and cultural settings may have an important role in these variations. Thus, the various and multiple claims about the therapeutic roles of medicinal plants during gestation indicate the need for further scientific investigations on these plants.

On the other hand, the study findings showed that the majority of women admitted to the hospital $(85.2 \%)$ used one or more prescription or non-prescription medicines (excluding vaccinations) prior to hospitalization. In line with previous studies from other geographical areas of Ethiopia [126], most of the pharmaceutical medicines used for self-medication were analgesics (Paper III) for the relief of pain or inflammation. This is corroborated by previous findings that Ethiopians employ self-medication for illnesses that are perceived as mild [34, 42]. Similarly, most of the prescription medicines used prior to hospitalization were antianaemic preparations, mainly ferrous sulphate (Paper IV). This too is in accordance with previous studies [150] indicating that there are similar reasons for use of medicines among anticipating women in the country. However, due care should be given as incorrect selfdiagnosis may delay seeking appropriate and timely healthcare for serious illnesses. Moreover, cautious use and physician's advice are important in preventing excessive dosage and longer 
duration, interaction between conventional medicines and medicinal plants and even pregnancy risks that OTC drugs carry.

Linum usitatissimum is by far the most widely used medicinal plant reported by pregnant women at JUMC. It was most commonly used for induction, reduction, quickening or preparation for labour (Paper II). This was in consonance with an ambulatory patient based study in central and northern Ethiopia which found similar reason for its use [149]. However, our finding is inconsistent with other studies which reported seed oil from $R$. communis plant as the most frequently recommended agent to stimulate labour $[3,187]$. The most probable reason for the disparity may be difference in geographical distribution of plants. In spite of its frequent use, literature indicates that there is inadequate scientific evidence about the therapeutic efficacy of L. usitatissimum for use in pregnancy $[188,189]$. However, in remote rural areas in Ethiopia where access to child-birthing health facilities is limited and any better options are not available, facilitation and induction of labour may be necessary in certain conditions; in this situation L. usitatissimum may be helpful. However, caution should still be exercised.

On the other hand, in accordance with the traditional claim reported in this study, human studies indicated that L. usitatissimum was found effective for the treatment of constipation due to its larger amount of insoluble fibre, and oil residue [188, 190]. Similar to other findings [191], pregnant women used it to manage signs and symptoms similar to irritable bowel syndrome and other pregnancy induced gastrointestinal disorders (Paper II). Moreover, its use for the management of abdominal cramps is supported by animal studies which showed its antispasmodic activity [192]. Flaxseed is also rich in fats, proteins, vitamins and minerals [188, 193]. Its possession of such a great number of therapeutic as well as nutritional benefits may be the reason for its wide utilization and might be beneficial to the mother if used carefully.

\subsubsection{Utilization pattern of medicinal plants}

When confronted with illnesses and discomfort, expectant mothers will look for medicinal plants and information from diverse sources. More or less consistent with results from many parts of developing and the developed world (Paper I), in this study family members recommended medicinal plant use to most women. In the same vein, the main aides for collecting medicinal plants were also family members (Paper II). These results are plausible as family members are easily reached, and usually the most trusted members of a community. 
However, as botanical medicines taken by the expectant mothers may cross the placenta and reach the developing foetus, it is concerning that women do not seek counselling from relevant professionals that can provide quality and reliable information. This indicates that pregnant women lack specific guidance on the use of plant remedies. Since studies have reported that women often do not communicate their use of medicinal plants to health care providers [194, 195], this could be a concern to the healthcare system. In addition, pregnant women should be informed that, similar to conventional medicines, herbal medicines have harmful effect if used in large quantities or frequencies and the belief that medicinal plants are natural and free of harm is not always the case.

Although standardization is a tool in the quality control process, at present no official standards are available for Ethiopian medicinal plants preparations [47]. For instance, this study showed that there is wide variation in utilization pattern such as wide range of dose, measures of formulation, frequency of administration, duration of treatment, episodes of use, among others (Paper I, Paper I I). Most medicinal plants are likely to cause harm, depending on the dose and duration of use [3, 23, 24]. Lack of standardization may expose the gestating woman or their unborn child to untoward effects, signifying that standardization of Ethiopian medicinal plants is a timely issue.

\subsubsection{Safety concerns of treatment modalities used by pregnant women}

\subsubsection{Safety concerns of medicinal plants}

Medicinal plants are often mistakenly viewed as natural and safe to use in pregnancy [19]. Unfortunately, this notion is dangerously misleading since some plants have toxic constituents and many have constituents with pharmacological activity, which could render them unsuitable during pregnancy $[25,146,196]$. Safety in pregnancy is well stablished only in ginger, but a few more are safe as long as they are used in moderation (Paper I). Based on information available in existing literature, three out of ten medicinal plants used by women in the present study (Paper II) were found to be potentially harmful to use during pregnancy. Hence, until compelling research evidence comes out, it is safest to consider particularly these potentially unsafe medicinal plants contraindicated in gestation. On the other hand, only less than a fifth $(18.5 \%)$ of the medicinal plants used by women were found safe to use in gestation (Paper II) based on current literature in our study; this is lower than findings from many other corners of the world $[23,24]$ warranting careful use among childbearing women and necessitating further research. 
There was not enough evidence that the most widely used plant was safe for use in pregnancy (Paper I). In addition, L. usitatissimum possesses chemicals that interact with nonsteroidal antiinflammatory drugs, anticoagulants, sulphonylureas and antiplatelets [188]. Besides, owing to its release of cyanide, the fresh L. usitatissimum plant is not recommended for expectant and breast-feeding mothers [191]. Considering the lack of adequate information about its safety and therapeutic efficacy, precautious consumption is important during pregnancy and lactation.

\subsubsection{Safety concerns and MRPs of conventional medicines}

It is reassuring that most of the conventional medicines used (prescribed or self-medicated) were probably safe to use during pregnancy because the majority are either supplements, analgesics or safer antimicrobials (Papers III, IV). This is corroborated by outpatient studies from different areas of Ethiopia [126, 150], indicating that there is similar pattern of medicine use in pregnancy across the country. However, some women carrying a child were selfmedicating with potentially risky medicines (Paper III) necessitating recommendations for cautious use, sticking to standard prescribing and dispensing guidelines and appropriate educational interventions.

In consonance with many previous studies [126] it is concerning that antimicrobials are among the top three groups of medicines used for self-medication in this study (Paper III). Furthermore, antimicrobials were irrationally used and gave rise to MRPs in the inpatient setting (Paper IV). Inappropriate use of antimicrobials is a huge problem in Ethiopia [197], and this indiscriminate use has led to many multidrug-resistant bacterial strains [197]. Moreover, over-the-counter sale of antimicrobials [38] and illegal sources of medicines [39] that are widespread in the country should be controlled by enforcing law and implementing stricter drug use policies. In general, efforts must be made to strengthen antimicrobial stewardship programmes.

Iron deficiency in pregnancy is responsible for numerous deleterious effects for both mother and infant especially in developing countries [198]. Even though the majority of the pregnant or lactating women were prescribed with iron sulphate during pregnancy (878/902, 97.3\%) (Paper IV), most hospitalized women were anaemic (haematocrit value $<33 \%$ or haemoglobin concentration $<11 \mathrm{~g} / \mathrm{dl}$ ) or were on the borderline. Additionally, nearly half of the encountered MRPs are due to iron sulphate treatment/supplementation and most of the MRPs that women experienced have moderate to severe potential to cause clinical deterioration (Paper IV). This 
finding is substantiated by studies that demonstrated anaemia is prevalent and iron deficiency anaemia is the most common form of anaemia in pregnancy in Ethiopia [199]. Access to ferrous sulphate alone does not guarantee adherence with treatment or a positive outcome and thus iron deficiency in pregnancy observed in this study might stem from several reasons as outlined below.

First is the adherence problem. Although women are prescribed with iron sulphate, they may not take it appropriately. In Ethiopia, the adherence rate to iron and folic acid supplementation is low mainly due to forgetfulness and fear of side effects including constipation [200]. Second, studies indicate that short gestation intervals are among the major risk factors for anaemia in Ethiopia [199]. This was consistent with our study, where a significant proportion of the study participants were multigravida and less than 30 years old (Paper II, Paper IV), and this could be another risk factor for anaemia. Third, some of the participants in this study were chronic khat chewers (Paper III) and chewing khat is reported to be an important predictor of medicinal plant use (Paper II) and anaemia during gestation in Ethiopia [160]. Therefore, chewing khat can result in iron deficiency which can be explained either by suppressing appetite [86, 160] or the inhibitory effect of khat on iron absorption. The khat plant is rich in tannin [86] which forms insoluble chelate in the gastrointestinal tract and obstructs bioavailability of non-heme iron. Fourth, clinical trials have established that consumption of phytate rich plants can lead to iron deficiencies [201]. Similarly, medicinal plant use in pregnancy was reported to be significantly associated with anaemia in Africa (Paper I). The most commonly used medicinal plant, $L$. usitatissimum, (Paper II) contains phytic acid, tannin and oxalate that binds with metallic elements to form insoluble complexes $[188,202]$, which can modulate iron absorption from the digestion tract [201]. Thus, extended consumption of tannin and phytate rich medicinal plants may chelate iron in the gut lumen and exacerbate iron deficiency. Fifth, iron deficiency anaemia is predictable [203] considering that Ethiopia is among the economically deprived nations in the globe with high rates of food insecurity and malnutrition [204]. Moreover, the lack of dietary diversity traditions might have effects on maternal burden of anaemia as there appears to be dependency on certain specific food crops in different parts of the country [203] which affects the iron status. In addition, particularly vegetables and fruits are consumed only infrequently and in small amounts in Ethiopia [203]. The problem is also compounded by HIV infections, or parasitic diseases which were reported by some women in this study (Paper III, IV). Finally, the forgetfulness or negligence from healthcare providers to screen for anaemia or 
to supply treatment and advice (Paper IV) could also contribute to the low haematocrit level and the observed MRP.

It is, therefore, imperative that healthcare professionals involved in maternal care advise women about the devastating effects of anaemia in pregnancy. Besides, they should give them appropriate advice to take iron/folic acid supplements during pregnancy. The client should be informed to adhere to the supplement treatment. Furthermore, healthcare professionals should make women aware of medicinal plant and social drug use and the pertinent precautions to be taken. Moreover, governmental and non-governmental bodies should exert efforts on iron sulphate/folic acid supplementation for all expectant mothers as part of the routine ANC.

One of the striking points this study found is that Anti-D immunoglobulin was the major factor leading to non-compliance type of MRPs (Paper IV). In consonance with most health facilities in Ethiopia [205], the common clinical practice in the study setting is to inject $300 \mu \mathrm{g}$ Anti-Rho (D) immune globulin doses to every Rhesus (Rh)-negative pregnant woman who could afford it within 72 hours of delivering an Rh-positive child. Although anti-D immunoglobulin was available in the study hospital, some women did not receive it due to financial problems (Paper IV). Evidence indicates that anti-D prophylaxis decreases the overall risk of $\mathrm{Rh}$ immunization from $13.2 \%$ to $0.2 \%$ [206]. Inaccessibility and unaffordability of anti-D immunoglobulin remain a significant problem in Sub-Saharan Africa [207]. To reduce maternal and neonatal morbidity and mortality, there is an urgent need for the implementation of a universal prophylactic anti-D access programme for all Rh-negative women who are pregnant. Healthcare workers should also exert the utmost possible effort to provide Rh-negative women the maximum standard of care before and after birth.

\subsubsection{Concomitant use of treatment modalities}

Concomitant use of different treatment modalities may result in potentially serious interactions leading to negative consequences on pregnancy outcomes [3, 149]. Therefore, herb-herb and drug-herb interactions are among the major concerns in the use of medicinal plants and conventional medicines in gestation (Paper I). In this study, women used various treatment modalities or social drugs simultaneously (Papers II, III, IV).

In addition to the use of one or more medicinal plants or orthodox medicines (Papers II, III, IV), some women reported consuming alcohol or khat on daily basis (Paper III). Medicinal 
plants contain complex mixtures of more than one pharmacologically active compounds which increase the likelihood of interactions [208-210]. Similarly, khat contains considerable amount of numerous chemical entities $[85,86]$ leading to increased risk of interaction. Hence, compared to conventional drugs which usually contain one active ingredient, the probability of herb-drug interactions is theoretically higher than drug-drug interactions [208]. In conformity with other African countries where different plant mixtures like isihlambezo are used (Paper I), Ethiopian women also used up to eight plants in singleton or in combinations for the treatment of various gestational ailments (Paper II). Synergistic and antagonistic interactions may produce unintended effects. Synergistic interactions, for example, between E. kebericho and $O$. lamiifolium are reported to worsen Mitch illness (Paper II). On top of the particularly vulnerable nature of pregnant women, the significant number of concomitant users found in this study (Papers II, III, IV) and the associated risks of interactions can further compromise the health of pregnant women and unborn children. Therefore, in the interest of foetal and maternal safety, healthcare professionals need to screen women for medicinal plant use or social drug consumption as an integrated part of ANC. Awareness should be created during women's health facility visits.

Apart from its inherent risks for both the mother and developing foetus [82], alcohol can also interact with several medications that can decrease the intended effects, render them useless or augment the harmful effects of the interacting medications [211-213]. Alcohol also has deleterious interactions with certain medicinal plants [212], further threatening maternal and foetal safety. In addition, most of the medications used for self-medication in the present study (Paper III) were indicated to have potentially serious medical interactions with alcohol [213] which can further exacerbate pregnancy induced illnesses and symptom complexes [212, 213]. To sum up, health practitioners need to be knowledgeable about such use and proactive in counselling pregnant women to avoid potential interactions between medicinal plants, pharmaceutical medicines and social drugs.

In addition to the inherent safety concerns of medicinal plants, there is also risk of contamination especially with heavy metals $[19,24,214]$ and adulteration with modern medicines [215]. Similar to a study from southern Ethiopia [19], over three out of four women in the present study accessed the medicinal plants from market places (Paper II). The poor regulatory framework for the production and distribution of medicinal plants [8, 19], coupled with the weak regulation [39] and irrational use of conventional medicines [38] in Ethiopia 
raises our concern about maternal and foetal safety. Considering this complex marketing process, the risk of contamination at some point in the supply chain cannot be ruled out necessitating careful use of medicinal plants, particularly among pregnant women.

\subsubsection{Factors associated with use of treatment modalities and MRPs}

This study found that various interconnected socio-demographic and maternal factors associated with use of treatment modalities. We found that women who practiced selfmedication were more likely to use medicinal plants and vice versa. Similarly, social drug use was a strong predictor of medicinal plant use and vice versa. Access to health facility was inversely associated with both medicinal plant use and self-medication. Adherents of Orthodox Christian or Islam religion were more likely to practice self-medication and use social drugs. Chronic disease was associated with both medicinal plant use and encounter of MRPs. Past medication use and parity were also strongly associated with encounter of MRPs.

Previous studies have reported that history of self-medication practice and history of disease were significantly associated with medicinal plant use [126]. Similarly, smokers were more likely to use herbs during pregnancy [216]. Access to health facilities near location of residence, and herbal medicine use experience [151,217] were reported predictors of selfmedication. Similar to previous findings, religion was related to self-medication [218]. However, it is not clear how religion affects self-medication and requires further investigations. In line with our finding, other studies have reported that prior multiple medication use and chronic disorder were significantly associated with experiencing an MRP [68, 69, 219]. Particularly, studies have indicated that chronic disease patients are more likely to use multiple medications making them prone to drug-drug interactions predisposing women to MRPs [159]. Therefore, intervention measures to reduce the harmful effects of medicines and herbs on pregnancy should consider this interrelated nature of predictor and outcome variables and focus on these prioritized areas.

\subsection{Discussion of the study methods}

This section presents methodological considerations that may apply in the interpretation of the results, and subsequent inference. This dissertation employed a systematic review and a hospital based cross-sectional study. Validity and reliability are concepts used to evaluate the quality of an epidemiological study. Validity is about the accuracy of a measure and reliability is about the consistency of a measure. Potential sources of errors that can affect validity and reliability 
of epidemiological studies are random or systematic. Since potential sources of error in a study should be acknowledged and deliberated, methodological issues in the four studies included in this dissertation that need to be considered are discussed in the following.

\subsubsection{Study designs}

This research project employed a systematic review (Paper I) and cross-sectional designs (Paper II, Paper III, Paper IV).

Systematic reviews seek to collate evidence that fits pre-specified eligibility criteria in order to answer a specific research question [220]. Systematic reviews are intended to render the highest level of evidence [221]. In Africa, data on prevalence of use, diversity, utilization, safety and efficacy of medicinal plants during pregnancy is still largely limited [222, 223]. Therefore, this systematic review (Paper I) is timely and involved a formal prespecified protocol with explicit, transparent scope of the question, criteria for eligibility, search strategy, selection and analysis of the studies, providing a more objective, and comprehensive overview of studies dealing about medicinal plants use during gestation. The exacting systematic approach of the review process reduced the potential bias in identifying the studies and made conclusions based on reliable results. In order to substantially minimize publication bias, unpublished studies, theses and dissertations were also included in the review. The review systematically assessed what is in the literature, identified the knowledge gap and suggested relevant measures to alleviate harmful effects on pregnancy. In addition to providing useful and systematically compiled evidence for health care workers, researchers and politicians, the review indicated future research focus areas. It also laid the foundation for subsequent studies included in this dissertation (particularly Paper II).

Cross-sectional studies describe the utilization of medicines in a given population [224], as represented by the study sample, at a given point in time or over a short period of days or weeks or months and thus are often called prevalence studies [224-227]. Cross-sectional studies are relatively inexpensive and easy to perform [228]. In a cross-sectional study, the population is assessed for both exposure and outcome at the same time [226, 229]. It is, therefore, important to acknowledge that since this study design lacks information on whether the treatment exposure precedes or follows the occurrence of the event, cross-sectional studies cannot assess the cause and effect relationship [225, 228, 230]. The association between the exposure and outcome is then assessed using measures of association. 
Cross-sectional study is the best design to assess population prevalence. It can be used to gather data on prescribing, dispensing or intake of medicine, and utilization patterns may be displayed by socio-demographic factors or geographical region [224]. In line with intent of this study design, the current study planned and assessed the prevalence of use of medicinal plants and pharmaceutical medicines among pregnant women. The response rate was high (98.6\%) and the incomplete data collection tools were only $4(0.4 \%)$, indicating that the effect of selection bias and non-response bias is minimal. Cross-sectional studies are retrospective in nature [226] which are conducted at a given point in time (Paper II, Paper III) or over a short period of months (Paper IV) and are prone to recall bias. However, to improve recall, many efforts were exerted in this study - including lists of commonly used medicinal plants and indications for use - to ensure that the effect of recall bias is negligible.

In summary, our use of systematic review study design (Paper I) and cross-sectional study design (Paper II, Paper III, Paper IV) are appropriate to answer the research questions.

\subsubsection{Study validity and reliability}

\subsubsection{Validity}

Validity is an expression of the degree to which a result from a study is likely to be true and free from bias [228, 231]. A study is valid if its results correspond to the truth; there should be no systematic error (bias) and the random error should be as small as possible [228]. There are two types of validity: internal validity (the extent to which the results of an observation are correct for the particular group of people being studied), and external validity or generalizability (the degree to which the results of a study can be generalized to the larger population from whom the study participants were drawn, i.e. the extent to which the sample represents the population) [228, 231]. Valid investigations require well-designed data collection tools, an appropriate sample of sufficient size, and a good response rate [228]. In accordance with this, several sources of error and bias and the methods we employed to minimize them in the papers included in this dissertation were duly addressed, as discussed below.

\subsection{Internal validity}

The major factors that affect internal validity are bias and confounding.

\subsection{Bias}




\section{Selection bias}

Selection bias arises when the characteristics of the study subjects lack representativeness of the target population about which conclusions are to be drawn [225] and consequently the prevalence of outcome variables and estimates of association in the study group differ from those in the target population [6]. We clearly defined the study population and used a clear and explicit list of inclusion criteria for the study in order to reduce the likelihood of selection bias. Moreover, data were collected by expert individuals who have knowledge of medicinal plants and conventional medicines used in the study area. This study also had a high response rate which minimized the possible selection bias. Furthermore, the enrolment of a large number of hospitalised pregnant women in a consecutive manner over a five-month period is believed to hinder selection bias. Therefore, we consider the chance of systematic selection in the sample to be small which will not lead to a biased estimate of the association between exposure and outcome. On the other hand, since the study facility is a referral hospital with a larger proportion of women with gestational complications, the included women may have a greater need for various treatment modalities and hence we cannot categorically exclude the risk of bias.

Selection bias was duly addressed in the systematic review through comprehensive search of print or digital studies and use of a standard abstracting form. A rigorous search of published articles, unpublished studies and grey literature was conducted to minimize the potential for publication bias. Language restriction was avoided. Studies were also traced through scrutinizing bibliographies. Research papers were also retrieved through personal contact of authors.

\section{Information bias}

Information bias is related to the way in which information about medicines and medicinal plant use and other study variables is collected. To ensure quality of the data collection, we have followed many strong steps using appropriate optimal instruments and data collection methods. First, because the specificity of questions about treatment use in pregnancy has implications for recall [6], we included commonly used names of medicinal plants and common indications in the data collection tool in order to improve recall and accuracy in the data collection process. Second, we also used open-ended questions to enable us gather all the necessary information. Third, experienced health professionals recruited from the study area collected the data. Fourth, data collectors were trained on each and every point included in the data collection tool so that they comprehended the questions themselves; they ensured that the respondents properly 
understood the questions, by repeating or rephrasing the questions before actual data collection. This is supported by the high response rate and almost no missing/incomplete questionnaires. Fifth, the data collection tools were pretested and reviewed by experts before they were used for actual data collection, and the interviewing skills of data collectors were assessed during pretesting. Sixth, to improve reporting and accuracy of the results, the interviews were performed in local languages, Amharic and Afan Oromo. Seventh, we followed careful and stringent data collection procedures including daily discussions with the data collectors and close monitoring by investigator. Finally, a panel of experts analysed the MRPs. Therefore, all of our methodological considerations and decisions to take the local situation into account have enabled us to improve recall and accuracy in reporting and have reduced the risk of information bias that could have emanated from poor study design. However, women may have reported medications and medicinal plants that they had taken recently or oftentimes in pregnancy rather than those they had used for short-term and few episodes; we recognize the possibility of recall bias.

\subsection{Confounding factors}

In a study of the association between a risk factor and the occurrence of outcomes under study, confounding can occur when another exposure exists in the study population and is associated (positively or negatively) both with the outcome and the exposure being studied [6,228]. These extraneous variables in a statistical model often bring about distortion of exposure-outcome association and so should be controlled [231]. To control confounding in the current study, stratification of certain variables (Table 1 in Papers II, III, IV) in to well defined and homogeneous categories was done in the analytical phase. Furthermore, multivariate logistic regression statistical modelling is used to estimate the strength of the associations of independent variables and the outcomes of study while controlling for several confounding variables concurrently. Thus, we believe that the impact of confounding that could have emanated from sociodemographic and pregnancy related variables is kept to a minimum in the outcomes of the current dissertation. Even though we have exerted great efforts to minimize confounding, the possibility of its presence cannot be rule out, for instance co-ingestion of treatments observed in this study could have a potential confounding effect.

\subsection{External validity}

External validity refers to the extent to which the study results can be generalized beyond the sample used in the study to a larger population from whom the study participants were drawn 
[231]. This usually depends on the degree to which the sample represents the population. Deviations of the sample measurement value from the true population value may be due to chance alone (also called variation) which is measured as random error [232, 233]. Random error causes inaccurate measures of associations [228]. Random error can never be completely eliminated since we can study only a sample of the population [228, 233], but it can be diminished by increasing the sample size of the study, and through replication of measurements [232]. To enhance generalizability, this study has employed a relatively large sample size of women that is believed to be large enough to reflect the actual composition of all the women's variables. This large sample size will help the study to have adequate statistical power to detect the differences considered important. Moreover, a statistical test was performed in order to address the role of chance by estimating CI. A p-value of less than or equal to 0.05 was declared statistically significant in the papers included in this dissertation. Nevertheless, the external validity of the current study may have been compromised by selecting a non-random consecutive hospitalized women who happened to be hospitalized during the study period. On the other hand, this study was conducted in a tertiary care teaching referral hospital, and, there is no guarantee that pregnant mothers who were hospitalized had similar morbidity, and medicinal plants and conventional medicines usage pattern compared to non-hospitalized pregnant population. Moreover, concerns remain about the study results' applicability to more diverse pregnant populations in the primary or secondary level hospitals, other public or private health facilities and rural or urban communities with larger proportion of pregnant women with fewer comorbidities and complications.

\subsubsection{Reliability}

Reliability is the degree to which the results can be reproduced when the study is repeated under comparable conditions. Random error can be reduced by replication of measurements [232]. The less variation in the yielded results in repeated measurements of an attribute the higher the reliability of the study and its results. However, poor reliability indicates either poor validity or that the characteristic that is being measured varies over time. In either of these circumstances results must be interpreted with caution. Since studies with strong external validity produce results that can be replicated across different contexts and time periods, we have followed rigorous data collection methods and analysis techniques which makes our study more reliable. Irrespective of the variations in the study setting and population, the findings of this study were found to be relatively comparable with other studies in terms of treatment use prevalence, safety and utilization pattern. Nevertheless, visible differences are observed. Therefore, more studies 
across time and across different observers should be conducted on the same topic in order to test replicability and robustness of research findings in diverse contexts and across time periods. Additional investigations should also check that study findings are generalizable, and the direction and strength of the investigated relationships is relatively similar.

\subsection{Strengths and limitations}

\section{Strengths}

This study has several methodological strengths. The study used both systematic review and cross-sectional study designs. To the best of my knowledge, this is the first comprehensive review to date of studies reporting medicinal plants used during pregnancy in Africa. It should be noted that the systematic review has followed strict procedures, among other things, to restrict selection and publication bias. In addition, the hospital based part is the most comprehensive study thus far to investigate and increase our understanding of many factors including medicinal plants, pharmaceutical medicines, and social drugs used during pregnancy, particularly in inpatient setting, that may affect pregnant women and their unborn children in Ethiopia.

To my knowledge, this is the first study to investigate medicinal plant use, self-medication and the safety profiles of treatments used during pregnancy among hospitalized women in Ethiopia. As yet no other study has also investigated MRPs among hospitalized pregnant women in Africa. In addition, the large sample size and high response rate of the study has provided greater insight into the used medicinal plants and medicines that might have potential harm for both the mother and developing foetus. The involvement of a panel of experts in MRPs identification, the use of questionnaires as well as the criteria for identification of MRPs that were evaluated and accepted by experts, the appraisal of severity level of MRPs, and the evaluation of safety profile of both medicinal plants (by relying on latest literature) and medicines (by using two pregnancy risk categorization systems from USA and Australia) were additional strengths of this study. The prospective identification of MRPs in hospitalized women also allowed for accurate evaluation of the clinical presentations and laboratory parameters, leading to quality data. That the participants were mainly women hospitalized to deliver their child enabled us to collect information about their experiences with their conventional medicine, and medicinal plants throughout pregnancy. The other strength of the current study lies in the face-to-face patient interview data collection method employed, which enabled the respondents to ask clarifying questions and ascertained completeness and 
comprehension. Finally, experienced health care professionals from the study area with knowledge about the local language, culture and health system conducted data collection which contributed toward the high response rate and quality data.

\section{Limitations}

The study has also limitations that should be acknowledged. Only cross-sectional studies were included in the review and thus their limitations would be reflected in the systematic review. Besides, the review focused only on studies reporting actual prevalence of use of medicinal plants among pregnant women, and thus a large number of qualitative and quantitative ethnobotanical studies reporting use of a particular plant species were excluded.

The self-reported medicinal plant and medication history may be limited by recall bias. Moreover, this study is hospital based and pregnant women might be confused or embarrassed about revealing usage of treatment modalities. Pregnant women may particularly underreport social drug use practices that are considered socially undesirable. Thus, it is worth noting that this could have influenced the outcome variables and identified predictors of outcome variables.

For ethical reasons, the healthcare workers were informed when MRPs were identified; this may have subsequently led to a decrease in the number of MRPs. For similar reasons, women with critical illnesses or other serious limiting conditions were excluded from the current study that lead to exclusion of complicated cases and in turn underestimation of the outcome variables of the study.

As the study setting is a teaching referral hospital with a greater share of women with pregnancy complications and possibly with a higher need for pharmaceutical medicines, it may not be fully representative of the pregnant population in different healthcare levels in the government or the private sector.

Some anticipating mothers had forgotten which modern medicines or medicinal plants and when and for which illness it was used; this may have affected the prevalence of the outcome variables as well as the corresponding predictor factors.

It should also be borne in mind that the accuracy of patient chart recordings were important constraints of this study. Due to lack of time, laboratory results or treatments administered to 
patients particularly in the maternity/labour ward operation room were often times not documented. The group of experts analysing the MRPs considered these as documentation problems instead of MRPs, which may have downplayed MRPs.

Despite these limitations, this study has generated new knowledge about pharmaceutical medicines and medicinal plants use during pregnancy in an area where scanty information exists. Therefore, the study should be extrapolated to other areas and settings with careful consideration of aforementioned strengths and limitations. 


\section{CONCLUSIONS AND IMPLICATIONS}

\subsection{Conclusions}

The study demonstrated that women use various medicinal plants, mostly Zingiber officinale (ginger) for the management of NVP, during pregnancy in Africa. The study also showed that although medicinal plants are commonly used throughout Africa, there is scarcity of research about their use in gestation. Moreover, there is limited data on the safety and efficacy of many medicinal plants used during pregnancy on the continent.

This study indicated that the use of medicinal plants during pregnancy is a common experience at JUMC in Ethiopia, mainly the use of L. usitatissimum (flaxseed) for induction or to reduce the intensity and shorten duration of labour. Furthermore, the use of medicinal plants is significantly variable and without standardization; this may result in adverse health consequences for both the mother and developing foetus.

Self-medication with pharmaceutical medicines, mainly with analgesics, is commonplace among pregnant women at JUMC. Even though the majority of conventional medicines used by pregnant women were probably safe to use during pregnancy, the majority of the medicinal plants used by women were found to be either potentially harmful or lacking in sufficient information about their use in pregnancy. Moreover, concomitant use of various treatment modalities or social drugs is common, and may lead to potentially harmful interactions.

The study also demonstrated that MRPs are a common phenomenon in the maternity and gynaecology wards at JUMC, predominantly in connection with the absence of iron supplementation. Furthermore, the significant proportion of the MRPs were found to be of moderate to severe potential, resulting in patient deterioration or discomfort.

Finally, the study showed that various interrelated socio-demographic and maternal factors significantly associated with use of treatment modalities, and occurrence of MRPs. This indicates that interventions to prevent pregnancy risks should focus on addressing multiple interdependent factors. Some of these interventions are described below.

\subsection{Implications for practice}

Health care professionals should be knowledgeable not only about conventional medicines but also about medicinal plants. They need to ask their pregnant patients about their use of both 
treatments and should provide the women with relevant evidence based advice as part of their routine care. Like orthodox medicines, health facilities also need to routinely include pregnant women's medicinal plants and social drug use history in patient records to optimize therapeutic benefits and prevent potential harm. In addition, health institutions should have a programme to counsel and provide health education about the potential benefits and risks of modern medicines and medicinal plants especially on the scopes of self-medication during pregnancy so that women can use them safely and have better pregnancy outcomes.

Since family members are indicated as agents for collection and recommendation of medicinal plants, government health education and promotion programmes on appropriate use of treatments should, in addition to targeting parturient population in hospitals, focus on other stakeholders including mothers, spouses, relatives and respected elderly persons in the community.

Adherence to the national standard treatment, prescribing and dispensing guidelines in maternity and obstetrics care is required in order to limit emerging microbial resistance, and occurrence of medication related problems. Likewise, a formal medication reconciliation programme should be designed and implemented for safe maternal care.

To reduce MRPs and mitigate their potential health risks, regular ward based clinical pharmacy services should be implemented. Similarly, collaboration between various healthcare professionals and patients should be strengthened, particularly in hospital settings, with the ultimate aim of minimizing risk and maximizing therapeutic benefit.

Finally, healthcare workers should provide dietary counselling and encourage women to consume iron /folate rich foods particularly during pregnancy.

\subsection{Implications for policy}

In spite of the fact that medicinal plants are extensively used by women in Ethiopia, these plants have not been incorporated into conventional medical practice. Thus, for better utilization and improved patient health, we recommend that the government integrate medicinal plant with the western healthcare system in the country. 
To raise the level of the national HEP, we recommend the training curriculum to include indigenous therapeutic approaches, dietary behaviours, rational medicine use and antimicrobial stewardship contents for better health of pregnant women as well as the community. Furthermore, special attention should be given to include indigenous healthcare practices in all healthcare professionals' training curricula in the country.

We recommend the Ethiopian government to introduce a mandatory fortification of widely consumed foods with iron (for example salt, edible oils, flour, grains) to facilitate iron access for all women who are pregnant and of reproductive age.

We recommend the Ministry of Health and Regional Health Bureaus of Ethiopia to give training to modern health care providers and education to the pregnant population and other stakeholders on responsible self-medication, medicinal plant use, social drug use and the associated risks. This can be done by using printed and electronic media and other means.

We recommend the Ministry of Health and Regional Health Bureaus of Ethiopia to ensure affordable access to health facilities and essential medicines for better maternal and childcare.

We recommend the government of Ethiopia to have a political commitment to effectively enforce the pharmaceutical legislation to prevent illegal sources of medicine. Similarly, we recommend the government of Ethiopia to effectively enforce the pharmaceutical legislation to prevent unauthorized prescribing and dispensing of medicines in authorized institutions thereby to prevent irrational use of medicines.

Finally, we recommend the government to involve the Women's Development Army in creating maternal awareness on appropriate use of treatment modalities to reach - particularly the rural women - more easily and quickly.

\subsection{Implications for future research}

We recommend further nationwide multicenter and multilevel studies to understand the frequency and nature of MRPs, medicinal plant use, self-medication practice, khat and alcohol use trends and determinant factors among the population of childbearing age to influence policy makers and effectively enforce the law. Our study is institutional, we recommend further 
community based research to identify the pattern and determinant factors of medication and medicinal plant usage among pregnant women.

We also recommend future research that focuses on establishing the safety and efficacy of the commonly used medicinal plants to ensure the well-being of gravid women and their developing foetus. In addition, future research could also be designed to specifically explore the efficacy of L. usitatissimum (flaxseed) in childbirth. Parallel to these, scientific investigations are necessary for validation of medicinal plants for the development of new therapeutic agents.

We also recommend future studies about healthcare professionals' adherence to clinical guidelines and patients' adherence to treatments, particularly iron sulphate treatment/supplementation, and associated underlying factors.

Finally, we recommend future investigations to focus on whether multidisciplinary health team collaborations and clinical pharmacy services in the obstetric in-patient setting can attenuate medication therapy problems, and if this brings about improvements in pregnancy outcomes. 


\section{REFERENCES}

1. WHO regional office for Africa: Traditional Medicine.

https://www.afro.who.int/health-topics/traditional-medicine. (2013). Accessed on 04 October 2020.

2. Witmer A, Seifer SD, Finocchio L, Leslie J, O'Neil EH: Community health workers: integral members of the health care work force. Am J Public Health 1995, $85(8 \mathrm{Pt}$ 1):1055-1058.

3. Ahmed SM, Nordeng H, Sundby J, Aragaw YA, de Boer HJ: The use of medicinal plants by pregnant women in Africa: A systematic review. J Ethnopharmacol 2018, 224:297-313.

4. McLay JS, Izzati N, Pallivalapila AR, Shetty A, Pande B, Rore C, Al Hail M, Stewart D: Pregnancy, prescription medicines and the potential risk of herb-drug interactions: a cross-sectional survey. BMC Complement Altern Med 2017, 17:543.

5. Shewamene Z, Dune T, Smith CA: The use of traditional medicine in maternity care among African women in Africa and the diaspora: a systematic review. BMC Complement Altern Med 2017, 17:382.

6. Nordeng H: Drug utilization in pregnant women. In: Drug Utilization Research: Methods and Applications. edn. Edited by Elseviers M, Wettermark B, Almarsdóttir AB, Andersen M, Benko R, Bennie M, Eriksson I, Godman B, Krska J, Poluzzi E et al. Chichester, West Sussex: Hoboken, NJ: John Wiley \& Sons Ltd; 2016: 240-247.

7. Namdeo AG: Chapter 20 - Cultivation of Medicinal and Aromatic Plants. In: Natural Products and Drug Discovery. edn. Edited by Mandal SC, Mandal V, Konishi T: Elsevier; 2018: 525-553.

8. Deribe K, Kassaye D, Amberbir A, Getachew B: A Historical Overview of Traditional Medicine Practices and Policy in Ethiopia. Ethiop J Health Dev 2006, 20(2): 127-134.

9. Pankhrust R: Historical reflection of traditional Ethiopian pharmacopoeia. Ethiop Pharm J 1976, 2: 29-32.

10. Suleman S, Beyene Tufa T, Kebebe D, Belew S, Mekonnen Y, Gashe F, Mussa S, Wynendaele E, Duchateau L, De Spiegeleer B: Treatment of malaria and related symptoms using traditional herbal medicine in Ethiopia. J Ethnopharmacol 2018, 213 : 262-279.

11. Bishaw M: Promoting traditional medicine in Ethiopia: a brief historical review of government policy. Soc Sci Med 1991, 33(2): 193-200. 
12. Fullas F: Interactions of Ethiopian Herbal Medicines and Spices with Conventional Drugs: A Practical Guide. South Sioux City, Nebraska: AJ Philipson Publishing Co.; 2006.

13. Ethiopian Public Health Institute (EPHI): Traditional and Modern Medicine Research http://www.ephi.gov.et/index.php/research/traditional-modern-medicine. (2013).

Accessed on 25 February 2019.

14. World Health Organization: WHO Global report on traditional and complementary medicine 2019. Geneva: World Health Organization; Licence: CC BY-NC-SA 3.0 IGO.

15. Abera B: Medicinal plants used in traditional medicine by Oromo people, Ghimbi District, Southwest Ethiopia. J Ethnobiol Ethnomed 2014, 10:40.

16. Yineger H, Yewhalaw D: Traditional medicinal plant knowledge and use by local healers in Sekoru District, Jimma Zone, Southwestern Ethiopia. J Ethnobiol Ethnomed 2007, 3:24.

17. Abbink J: Medicinal and ritual plants of the Ethiopian Southwest: an account of recent research. IK\&DM 1995, 3:6-8.

18. Mekonnen A, Bluffstone R: Policies to increase forest cover in Ethiopia: lessons from economics and international experience. In: Policies to increase forest cover in Ethiopia, In: Proceedings of a Policy Workshop organized by Environmental Economics Policy Forum for Ethiopia (EEPFE) of Ethiopian Development Research Institute (EDRI), Addis Ababa, Ethiopia; 2007, 23-64.

19. Laelago T, Yohannes T, Lemango F: Prevalence of herbal medicine use and associated factors among pregnant women attending antenatal care at public health facilities in Hossana town, southern Ethiopia: facility based cross sectional study. Arch Public Health 2016, 74 :7.

20. Servey J, Chang J: Over-the-Counter Medications in Pregnancy. Am Fam Physician 2014, 90(8): 548-555.

21. Sachdeva P, Patel BG, Patel BK: Drug use in pregnancy; a point to ponder! Indian J Pharm Sci 2009, 71: 1-7

22. Porter RS: The Merck Manual's Online Medical Library. Whitehouse Station: Merck Research Lab; 2004.

23. Kennedy DA, Lupattelli A, Koren G, Nordeng H: Safety classification of herbal medicines used in pregnancy in a multinational study. BMC Complement Altern Med 2016, 16:102. 
24. Ahmed M, Hwang JH, Choi S, Han D: Safety classification of herbal medicines used among pregnant women in Asian countries: a systematic review. BMC Complement Altern Med 2017, 17:489.

25. Dugoua J-J: Herbal medicines and pregnancy. J Popul Ther Clin Pharmacol 2010, 17(3): e370-8.

26. Hall HG, Griffiths DL, McKenna LG: The use of complementary and alternative medicine by pregnant women: A literature review. Midwifery 2011, 27(6):817-824.

27. Westfall RE: Herbal healing in pregnancy: women's experiences. J Herb Pharmacother 2003, 3.

28. Kebede B, Gedif T, Getachew A: Assessment of drug use among pregnant women in Addis Ababa, Ethiopia. Pharmacoepidemiol Drug Saf 2009, 18: 462-468.

29. Andrade SE, Gurwitz JH, Davis RL, Chan KA, Finkelstein JA, Fortman K, McPhillips H, Raebel MA, Roblin D, Smith DH et al: Prescription drug use in pregnancy. Am J Obstet Gynecol 2004, 191: 398-407.

30. State Government of Victoria, Department of Health \& Human Services. Pregnancy medication, drugs and alcohol

[https://www.betterhealth.vic.gov.au/health/healthyliving/pregnancy-medicationdrugs-and-alcohol]. (2012). Accessed on 06 October 2020.

31. World Health Organization: Guidelines for the regulatory assessment of medicinal products for use in self-medication. Geneva, Switzerland: World Health Organization; 2000 .

32. Ebrahimi H, Atashsokhan G, Amanpour F, Hamidzadeh A: Self-medication and its risk factors among women before and during pregnancy. Pan Afr Med J 2017, 27: 183.

33. Ahmed SM, Sundby J, Aragaw YA, Abebe F: Self-Medication and Safety Profile of Medicines Used among Pregnant Women in a Tertiary Teaching Hospital in Jimma, Ethiopia: A Cross-Sectional Study. Int J Environ Res Public Health 2020, 17(11): 3993.

34. Ayalew MB: Self-medication practice in Ethiopia: a systematic review. Patient Prefer Adherence 2017, 11: 401-413.

35. Ansari M: Chapter 2 - Sociobehavioral Aspects of Medicines Use in Developing Countries. In: Social and Administrative Aspects of Pharmacy in Low- and MiddleIncome Countries. edn. Edited by Ibrahim MIM, Wertheimer AI, Babar Z-U-D: Academic Press; 2018: 15-33. 
36. Marwa KJ, Njalika A, Ruganuza D, Katabalo D, Kamugisha E: Self-medication among pregnant women attending antenatal clinic at Makongoro health centre in Mwanza, Tanzania: a challenge to health systems. BMC Pregnancy Childbirth 2018, 18: 16 .

37. BiBintsene-Mpika G, Mouankié JB, Ndziessi G, Mozoma LO, Iloki LH, AA A: Selfmedication practice among pregnant women in Brazzaville. Int J Fam Commun Med 2018, 2(3):137-140.

38. Beyene KGM, Beza SW: Self-medication practice and associated factors among pregnant women in Addis Ababa, Ethiopia. Trop Med Health 2018, 46:10.

39. Suleman S, Woliyi A, Woldemichael K, Tushune K, Duchateau L, Degroote A, Vancauwenberghe R, Bracke N, De Spiegeleer B: Pharmaceutical Regulatory Framework in Ethiopia: A Critical Evaluation of Its Legal Basis and Implementation. Ethiop J Health Sci 2016, 26(3): 259-276.

40. Rahmani A, Hamanajm S, Fallahi A, Gheshlagh R, Dalvand S: Prevalence of selfmedication among pregnant Women: A systematic review and meta-analysis. Nurs Midwifery Stud 2019, 8(4): 169-175.

41. Mohseni M, Azami-Aghdash S, Gareh Sheyklo S, Moosavi A, Nakhaee M, Pournaghi-Azar F, Rezapour A: Prevalence and Reasons of Self-Medication in Pregnant Women: A Systematic Review and Meta-Analysis. Int J Community Based Nurs Midwifery 2018, 6(4): 272-284.

42. Sisay M, Mengistu G, Edessa D: Epidemiology of self-medication in Ethiopia: a systematic review and meta-analysis of observational studies. BMC Pharmacol Toxicol 2018, 19: 56.

43. Holst L, Wright D, Haavik S, Nordeng H: Safety and efficacy of herbal remedies in obstetrics—review and clinical implications. Midwifery 2011, 27(1):80-86.

44. Rouamba T, Valea I, Bognini JD, Kpoda H, Mens PF, Gomes MF, Tinto H, KirakoyaSamadoulougou F: Safety Profile of Drug Use During Pregnancy at Peripheral Health Centres in Burkina Faso: A Prospective Observational Cohort Study. Drugs Real World Outcomes 2018, 5(3):193-206.

45. Joseph B, Ezie I, Aya B, Dapar M: Self-medication among pregnant women attending ante-natal clinics in Jos-North, Nigeria. Int J Trop Dis Health 2017, 21(1):1-7.

46. Kebede B, Gedif T, Getachew A: Assessment of drug use among pregnant women in Addis Ababa, Ethiopia. Pharmacoepidemiol Drug Saf 2009, 18(6):462-468. 
47. Jambo A, Mengistu G, Sisay M, Amare F, Edessa D: Self-Medication and Contributing Factors Among Pregnant Women Attending Antenatal Care at Public Hospitals of Harar Town, Ethiopia. Front Pharmacol 2018, 9:1063.

48. Tronnes JN, Lupattelli A, Nordeng H: Safety profile of medication used during pregnancy: results of a multinational European study. Pharmacoepidemiol Drug Saf 2017, 26(7):802-811.

49. Koren G, Pastuszak A, Ito S: Drugs in pregnancy. N Engl J Med 1998, 338(16):11281137.

50. Kim JH, Scialli AR: Thalidomide: the tragedy of birth defects and the effective treatment of disease. Toxicol Sci 2011, 122(1):1-6.

51. Black RA, Hill DA: Over-the-counter medications in pregnancy. Am Fam Physician 2003, 67(12):2517-2524.

52. Läkemedelsindustriföreningen. FASS for vårdpersonal. http://www.fass.se/LIF/startpage. Accessed on 03 September 2020.

53. Sannerstedt R, Lundborg P, Danielsson BR, Kihlström I, Alván G, Prame B, Ridley E: Drugs During Pregnancy. Drug Saf 1996, 14(2):69-77.

54. Olesen C, Sorensen HT, de Jong-van den Berg L, Olsen J, Steffensen FH, The Euromap G: Prescribing during pregnancy and lactation with reference to the Swedish classification system. A population-based study among Danish women. Acta Obstet Gynecol Scand 1999, 78(8):686-692.

55. US Food and Drug Administration(FDA): Pregnancy and Lactation Labeling (Drugs) Final Rule. https://www.fda.gov/drugs/labeling-information-drug-products/pregnancyand-lactation-labeling-drugs-final-rule. Accessed on 27 September 2019.

56. Australian Government Department of Health: Therapeutic Good Administration: Australian categorisation system for prescribing medicines in pregnancy. https://www.tga.gov.au/australian-categorisation-system-prescribing-medicinespregnancy. Accessed on 19 November 2019.

57. Food Medicine and Healthcare Administration and Control Authority (FMHACA): Standard Treatment Guideline for Primary Hospital, 3rd edn. Addis Ababa, Ethiopia: FMHACA; 2014.

58. Food Medicine and Healthcare Administration and Control Authority (FMHACA): Standard Treatment Guideline for General Hospital, 3rd edn. Addis Ababa, Ethiopia: FMHACA; 2014. 
59. Hepler CD, Strand LM: Opportunities and responsibilities in pharmaceutical care. Am J Hosp Pharm 1990, 47(3):533-543.

60. Cipolle R, Strand L, Morley P: Pharmaceutical Care Practice: The Patient-Centered Approach to Medication Management Services, 3rd edn: New York: McGraw-Hill; 2012.

61. Pharmaceutical Care Network Europe Association 2003-2019. PCNE Classification for Drug-Related Problems V9.00. 2019.

https://www.pcne.org/upload/files/334_PCNE_classification_V9-0.pdf. Accessed on 20 September 2019.

62. Cipolle RJ SL, Morley PC: Pharmaceutical Care Practice: The clinician's guide., 2nd edn. New York: McGraw-Hill; 2004.

63. Johnson JA, Bootman JL: Drug-related morbidity and mortality. A cost-of-illness model. Arch Intern Med 1995, 155(18):1949-1956.

64. Al-Arifi M, Abu-Hashem H, Al-Meziny M, Said R, Aljadhey H: Emergency department visits and admissions due to drug related problems at Riyadh military hospital (RMH), Saudi Arabia. Saudi Pharm J 2014, 22(1):17-25.

65. Watanabe J, McInnis T, Hirsch J: Cost of Prescription Drug-Related Morbidity and Mortality. Ann Pharmacother 2018, 52(9):829-837.

66. van Mil JW, Westerlund LO, Hersberger KE, Schaefer MA: Drug-related problem classification systems. Ann Pharmacother 2004, 38(5):859-867.

67. Björkman IK, Sanner MA, Bernsten CB: Comparing 4 classification systems for drugrelated problems: processes and functions. Res Social Adm Pharm 2008, 4(4):320331.

68. Thompson R, Whennan L, Liang J, Alderman C, Grzeskowiak L: Investigating the Frequency and Nature of Medication-Related Problems in the Women's Health Unit of an Australian Tertiary Teaching Hospital. Ann Pharmacother 2015, 49(7):770-776.

69. Smedberg J, Brathen M, Waka MS, Jacobsen AF, Gjerdalen G, Nordeng H: Medication use and drug-related problems among women at maternity wards-a crosssectional study from two Norwegian hospitals. Eur J Clin Pharmacol 2016, 72(7):849857.

70. Wilmer CM, Huiskes VJB, Natsch S, Rennings AJM, van den Bemt BJF, Bos JM: Drug-related problems in a clinical setting: a literature review and cross-sectional study evaluating factors to identify patients at risk. Eur J Hosp Pharm 2015, 22(4):229-235. 
71. Freyer A: Drug-prescribing challenges during pregnancy. Obstet Gynaecol Reprod Med 2008, 18:180-186.

72. Kumar S, Dahal P, Venkataraman R, Fuloria PC: Assessment of clinical pharmacist intervention in tertiary care teaching hospital of Southern India. Asian J Pharm Clin Res 2013, 6:258-261.

73. Cornish PL, Knowles SR, Marchesano R, Tam V, Shadowitz S, Juurlink DN, Etchells EE: Unintended medication discrepancies at the time of hospital admission. Arch Intern Med 2005, 165(4):424-429.

74. Pangle B: Drugs in Pregnancy and Lactation. In: Text book of Therapeutics, Drug and Disease Management 8th edn. Edited by Herfindal E, Gourley D. Philadelphia: Lippincott Williams \& Wilkins; 2006: 434-448.

75. Central Statistical Agency (CSA) [Ethiopia] and ICF: Ethiopian Demographic and Health Survey, 2016. Addis Ababa, Ethiopia, and Rockville, Maryland, USA: CSA and ICF; 2017.

76. Ministry of Health - Ethiopia: Essential Health Services Package of Ethiopia. Addis Ababa, Ethiopia: Ministry of Health of Ethiopia; 2019.

77. World Health Organization: WHO recommendations on prevention and management of tobacco use and second-hand smoke exposure in pregnancy - The short version. Geneva: WHO; 2014.

78. Aderaw A: Caffeine, Alcohol, Khat, and Tobacco use during pregnancy in Butajira, south central Ethiopia (M.Sc. Thesis). Addis Ababa University, College of Health science, School of Public Health, Addis Ababa, Ethiopia; 2018. http://etd.aau.edu.et/handle/123456789/14442. Accessed on 07 February 2020.

79. Mohammed AM, Ahmed JH, Bushra AW, Aljadhey H: Medications use among pregnant women in Ethiopia: A cross sectional study. J Appl Pharm Sci 2013, 3(4):116-123.

80. World health organization: Global status report on alcohol and health. Geneva, Switzerland: WHO; 2018.

81. Popova S, Lange S, Probst C, Gmel G, Rehm J: Estimation of national, regional, and global prevalence of alcohol use during pregnancy and fetal alcohol syndrome: a systematic review and meta-analysis. Lancet Glob Health 2017, 5(3):e290-e299.

82. Popova S, Lange S, Probst C, Shield K, Kraicer-Melamed H, Ferreira-Borges C, Rehm J: Actual and predicted prevalence of alcohol consumption during pregnancy in the WHO African Region. Trop Med Int Health 2016, 21(10):1209-1239. 
83. Fekade M: Alcohol use and associated factors during pregnancy at Kolfe Keraniyo sub city in Addis Ababa, Ethiopia (M.Sc. Thesis). Addis Ababa University, College of Health science, School of Public Health, Addis Ababa, Ethiopia; 2017. http://etd.aau.edu.et/bitstream/handle/123456789/14326/Maereg\%20Fekade.pdf?seque nce $=1 \&$ isAllowed=y. Accessed on 13 February 2020.

84. Addila AE, Bisetegn TA, Gete YK, Mengistu MY, Beyene GM: Alcohol consumption and its associated factors among pregnant women in Sub-Saharan Africa: a systematic review and meta-analysis. Subst Abuse Treat Prev Policy 2020, 15:29.

85. Abate K: Khat (Catha edulis) chewing as a risk factor of low birth weight among full term Newborns: A systematic review. Middle East J Fam Med 2015, 13(7):10-14.

86. Engidawork E: Pharmacological and Toxicological Effects of Catha edulis F. (Khat). Phytother Res 2017, 31(7):1019-1028.

87. Nakajima M, Jebena MG, Taha M, Tesfaye M, Gudina E, Lemieux A, Hoffman R, al'Absi M: Correlates of khat use during pregnancy: A cross-sectional study. Addict Behav 2017, 73:178-184.

88. Feyissa AM, Kelly JP: A review of the neuropharmacological properties of khat. Prog Neuropsychopharmacol Biol Psychiatry 2008, 32(5):1147-1166.

89. Jansson T, Kristiansson B, Qirbi A: Effect of khat on uteroplacental blood flow in awake, chronically catheterized, late-pregnant guinea pigs. J Ethnopharmacol 1988, 23:19-26.

90. Selassie SG, Gebre A: Rapid assessment of drug abuse in Ethiopia. Bull Narc 1996, 48(1-2):53-63.

91. Mekuriaw B, Belayneh Z, Yitayih Y: Magnitude of Khat use and associated factors among women attending antenatal care in Gedeo zone health centers, southern Ethiopia: a facility based cross sectional study. BMC Public Health 2020, 20:110.

92. Federal Democratic Republic of Ethiopia: Federal Negarit Gazette of the Federal Democratic Republic of Ethiopia, Number 39, 28 February 2019. Food and Medicine Administration Proclamation, Proclamation No. 1112/2019. Addis Ababa, Ethiopia.

93. Dahab R, Sakellariou D: Barriers to Accessing Maternal Care in Low Income Countries in Africa: A Systematic Review. Int J Environ Res Public Health 2020, 17(12):4292.

94. World Health Organization. Maternal health. https://www.who.int/healthtopics/maternal-health\#tab=tab_1. Accessed on 19 October 2020. 
95. Eshiet I: Sustainable Development Goal 3 and Maternal Health in Nigeria: Any Hope of Meeting the Target by 2030? In: Innovations in Global Maternal Health: Improving Prenatal and Postnatal Care Practices. edn. Edited by Association IRM. Hershey, PA: IGI global; 2020.

96. World Health Organization. Maternal mortality. https://apps.who.int/iris/bitstream/handle/10665/329886/WHO-RHR-19.20eng.pdf?ua=1. Accessed on 29 September 2020.

97. World Health Organization regional office for Europe. Maternal and newborn health. https://www.euro.who.int/en/health-topics/Life-stages/maternal-and-newbornhealth/maternal-and-newbornhealth\#: :text=Maternal\%20health\%20refers\%20to\%20the,babies $\% 20$ first $\% 20$ month $\% 20 \mathrm{of} \% 201$ ife. Accessed on 23 October 2020.

98. World Health Organization. Maternal mortality. https://www.who.int/news-room/factsheets/detail/maternal-mortality. Accessed on 23 September 2020.

99. World health organization: Trends in maternal mortality 2000 to 2017: estimates by WHO, UNICEF, UNFPA, World Bank Group and the United Nations Population Division. Geneva,Switzerland World Health Organization; Licence: CC BY-NC-SA 3.0 IGO.; 2019.

100. The United States Agency for International Development (USAID): Cultural Barriers to Seeking Maternal Health Care in Ethiopia: A Review of the Literature. Addis Ababa, Ethiopia: The United States Agency for International Development (USAID) and Maternal and Child Health Integrated Program (MCHIP); 2012.

101. UNICEF. Millennium Development Goals:5. Improve Maternal Health. http://www.unicef.org/mdg/maternal.html. Accessed on 23 November 2019.

102. Federal Democratic Republic of Ethiopia Ministry of Health: HSTP Health Sector Transformation Plan. 2015/16 - 2019/20 (2008-2012 EFY). Addis Ababa, Ethiopia: Ministry of Health; 2015.

103. British Broadcasting Corporation (BBC). Ethiopia country profile. https://www.bbc.com/news/world-africa-13349398. Accessed on 20 August 2020.

104. Federal Democratic Republic of Ethiopia Ministry of Health: Health Sector Development Program IV, 2010/11 - 2014/15. Addis Ababa, Ethiopia: Ministry of Health; 2010.

105. Federal Democratic Republic of Ethiopia Ministry of Foreign Affairs. The Federal Democratic Republic of Ethiopia (FDRE). 
http://www.mfa.gov.et/Home/TemplateI?PageName=Profile\&TYPE=About\%20Ethio pia\&SUBTYPE=About\%20Ethiopia\&Language=English. Accessed on 18 September 2020.

106. Ethiopian treasures: Ethiopian Geography. http://www.ethiopiantreasures.co.uk/pages/geography.htm. Accessed on 13 October 2020

107. Huihui W, Tesfaye R, Ramana G, Chekagn C: Ethiopia Health Extension Program: An Institutionalized Community Approach for Universal Health Coverage: World Bank Studies. Washington, DC: World Bank; 2016.

108. United States CIA: The World Fact Book, Ethiopia. https://www.cia.gov/library/publications/the-world-factbook/geos/et.html. Accessed on 13 February 2019.

109. World Food Programme (WFP): Ethiopia country strategic plan (2020-2025). Rome, Italy; 2020.

110. Ethiopian Public Health Institute (EPHI) [Ethiopia] and ICF: Ethiopia Mini Demographic and Health Survey 2019: Key Indicators. Rockville, Maryland, USA: EPHI and ICF; 2019.

111. Federal Ministry of Health of Ethiopia: Health and Health Related Indicators 2005 E.C (2012/2013), Version 2. Addis Ababa, Ethiopia: Ministry of Health; 2014.

112. World Health Organization. World Health Organization Health Action in Crises Ethiopia Strategy Paper. https://www.who.int/hac/crises/eth/Ethiopia_strategy_document.pdf. Accessed on 27 October 2020.

113. Food and Agriculture Organization: AQUASTAT Country Profile - Ethiopia. Rome, Italy: FAO; 2016.

114. Teshome A, Lupi A: Determinants of Agricultural Gross Domestic Product in Ethiopia. Int J Res Stud Agric Sci 2018, 4(2):12-20.

115. Khatami R, Southworth J, Muir C, Caughlin T, Ayana AN, Brown DG, Liao C, Agrawal A: Operational Large-Area Land-Cover Mapping: An Ethiopia Case Study. Remote Sens 2020, 12(6):954.

116. World Bank Group: Data: Ethiopia. https://data.worldbank.org/country/ethiopia?view=chart. Accessed on 25 October 2020 . 
117. Regassa N: Antenatal and postnatal care service utilization in southern Ethiopia: a population-based study. Afr Health Sci 2011, 11(3):390-397.

118. Federal Ministry of Health (FMOH) and Global Health Supply Chain-Procurement and Supply Management (GHSC-PSM): National Clinical Pharmacy Service Implementation Manual in Ethiopia. Addis Ababa, Ethiopia: Ministry of Health; 2018.

119. Federal democratic republic of Ethiopia Ministry of Health: National Pharmacy Service, Pharmaceuticals Supply Cain and Media Equipment Managment Monitering and Evaluation Framework. Addis Ababa, Ethiopia: Ministry of Health; 2019.

120. Eregata G, Hailu A, Memirie S, Norheim O: Measuring progress towards universal health coverage: national and subnational analysis in Ethiopia. BMJ Glob Health 2019, 4(6):e001843.

121. World Health Organization. Alma Ata Declaration 1978. http://www.euro.who.int/_data/assets/pdf_file/0009/113877/E93944.pdf. Accessed on 10 September 2020.

122. Assefa Y, Gelaw YA, Hill PS, Taye BW, Van Damme W: Community health extension program of Ethiopia, 2003-2018: successes and challenges toward universal coverage for primary healthcare services. Glob Health 2019, 15(1):24.

123. Damtew ZA, Lemma S, Zulliger R, Moges AS, Teklu A, Perry HB. Ethiopia's Health Extension Program. https://chwcentral.org/ethiopias-health-extension-program/. Accessed on 06 October 2020.

124. United Nations Development Programme: Millennium Development Goals: Improve maternal health, where we are?

https://www.et.undp.org/content/ethiopia/en/home/SDG/mdgoverview/overview/mdg5 1. Accessed on 22 August 2020.

125. Central Statistical Agency [Ethiopia] and ICF International: Ethiopia Demographic and Health Survey 2011. Addis Ababa, Ethiopia and Calverton, Maryland, USA: Central Statistical Agency and ICF International; 2012.

126. Adane F, Seyoum G, Alamneh YM: Non-prescribed drug use and predictors among pregnant women in Ethiopia: systematic review and meta-analysis. J Matern Fetal Neonatal Med 2020:1-12.

127. Central Statistical Authority [Ethiopia] and ORC Macro x: Ethiopia Demographic and Health Survey 2000. Addis Ababa, Ethiopia and Calverton, Maryland, USA: Central Statistical Agency and ORC Macro; 2001. 
128. Central Statistical Agency [Ethiopia] and ORC Macro: Ethiopia Demographic and Health Survey 2005. Addis Ababa, Ethiopia and Calverton, Maryland, USA: Central Statistical Agency and ORC Macro; 2006.

129. Millennium Development Goals (MDGs). https://www.who.int/topics/millennium_development_goals/about/en/. Accessed on 22 August 2020.

130. Banteyerga H: Ethiopia's health extension program: improving health through community involvement. MEDICC Rev 2011, 13(3):46-49.

131. Assefa Y, Damme WV, Williams OD, Hill PS: Successes and challenges of the millennium development goals in Ethiopia: lessons for the sustainable development goals. BMJ Glob Health 2017, 2(2):e000318.

132. United Nations Development Programme: Sustainable development goals. https://www.undp.org/content/undp/en/home/sustainable-development-goals.html. Accessed on 22 August 2020.

133. Federal democratic republic of Ethiopia Ministry of Health: 2nd Generation Health Extension Program to be implemented in 4,500 Clinics. http://www.moh.gov.et/ejcc/index.php/en/node/177. Accessed on 22 August 2020.

134. Federal Democratic Republic of Ethiopia Ministry of Health. Second Generation Health Extension Program.

https://publications.jsi.com/JSIInternet/Inc/Common/_download_pub.cfm?id=16833\& lid=3. Accessed on 22 August 2020 .

135. World Health Organization: World health statistics 2020: monitoring health for the SDGs, sustainable development goals. Geneva, Switzerland: World Health Organization; 2020.

136. Federal Democratic Republic of Ethiopia National Planning Commission: Growth and Transformation Plan II (GTP II) (2015/16-2019/20). Addis Ababa, Ethiopia: National Planning Commission; 2016.

137. Federal Democratic Republic of Ethiopia Ministry of Health: Addressing the impact of noncommunicable diseases and injuries in Ethiopia: findings and recommendations from the noncommunicable diseases and injuries (NCDI) commission of Ethiopia. Addis Ababa, Ethiopia: Ethiopia NCDI Commission Report, a Collaboration with the Global Lancet Commission on Reframing NCDIs for the Poorest Billion; 2018.

138. Federal Democratic Republic of Ethiopia Ministry of Health: Programs \& Projects. http://www.moh.gov.et/ejcc/en/node/20. Accessed on 22 August 2020. 
139. Misganaw A, Haregu TN, Deribe K, Tessema GA, Deribew A, Melaku YA, Amare AT, Abera SF, Gedefaw M, Dessalegn M et al: National mortality burden due to communicable, non-communicable, and other diseases in Ethiopia, 1990-2015: findings from the Global Burden of Disease Study 2015. Popul Health Metr 2017, $15: 29$.

140. Federal Democratic Republic of Ethiopia Ministry of Health: Health and Health Related Indicator 2009 EFY (2016/2017). Addis Ababa, Ethiopia: Ministry of Health; 2017.

141. Ahmed M, Hwang JH, Hasan MA, Han D: Herbal medicine use by pregnant women in Bangladesh: a cross-sectional study. BMC Complement Altern Med 2018, 18(1):333333.

142. Kennedy DA, Lupattelli A, Koren G, Nordeng H: Herbal medicine use in pregnancy: results of a multinational study. BMC Complement Altern Med 2013, 13:355-355.

143. Cordier W, Steenkamp V: Drug interactions in African herbal remedies. Drug Metabol Drug Interact 2011, 26(2):53-63.

144. El Hajj M, Holst L: Herbal Medicine Use During Pregnancy: A Review of the Literature With a Special Focus on Sub-Saharan Africa. Front Pharmacol 2020, $11: 866$.

145. Fugh-Berman A, Ernst E: Herb-drug interactions: Review and assessment of report reliability. Br J Clin Pharmacol 2001, 52(5):587-595.

146. Adane F, Seyoum G, Alamneh YM, Abie W, Desta M, Sisay B: Herbal medicine use and predictors among pregnant women attending antenatal care in Ethiopia: a systematic review and meta-analysis. BMC Pregnancy Childbirth 2020, 20:157.

147. Bayisa B, Tatiparthi R, Mulisa E: Use of herbal medicine among pregnant women on antenatal care at Nekemte Hospital, Western Ethiopia. Nat Pharm Prod 2014, 9(4):e17368.

148. Mekuria AB, Erku DA, Gebresillassie BM, Birru EM, Tizazu B, Ahmedin A: Prevalence and associated factors of herbal medicine use among pregnant women on antenatal care follow-up at University of Gondar referral and teaching hospital, Ethiopia: a cross-sectional study. BMC Complement Altern Med 2017, 17:86.

149. Nega SS, Bekele HM, Meles GG, Nordeng H: Medicinal Plants and Concomitant Use with Pharmaceutical Drugs Among Pregnant Women. J Altern Complement Med 2019, 25(4):427-434. 
150. Ayele Y, Mekuria AN, Tola A, Mishore KM, Geleto FB: Prescription drugs use during pregnancy in Ethiopia: A systematic review and meta-analysis. SAGE Open Med 2020, 8:1-10.

151. Befekadu A, Dhekama NH, Mohammed MA: Self-medication and Contributing Factors among Pregnant Women Attending Antenatal Care in Ethiopia: The Case of Jimma University Specialized Hospital. Med-Science 2014, 3(1):969-981.

152. Abeje G, Admasie C, Wasie B: Factors associated with self medication practice among pregnant mothers attending antenatal care at governmental health centers in Bahir Dar city administration, Northwest Ethiopia, a cross sectional study. Pan Afr Med J 2015, 20:276-276.

153. Gebremedhin GL, Gomathi P: Assessment of Drug Use and Effect in Pregnant Women Attending Antenatal Care in Hospitals of Mekelle, Tigray, Ethiopia. J drug deliv ther 2014, 4( 6):75-82.

154. Wakjira G, Boru B, Labata B: Prevalence of self-medication and its associated factors among pregnant women attending antenatal care at Nekemte Referral Hospital, Oromia Regional State, West Ethiopia. J Bioanal Biomed 2019, 11:160-165.

155. Birarra MK, Heye TB, Shibeshi W: Assessment of drug-related problems in pediatric ward of Zewditu Memorial Referral Hospital, Addis Ababa, Ethiopia. Int J Clin Pharm 2017, 39(5):1039-1046.

156. Yimama M, Jarso H, Desse TA: Determinants of drug-related problems among ambulatory type 2 diabetes patients with hypertension comorbidity in Southwest Ethiopia: a prospective cross sectional study. BMC Res Notes 2018, 11(1):679.

157. Tigabu BM, Daba D, Habte B: Drug-related problems among medical ward patients in Jimma university specialized hospital, Southwest Ethiopia. J Res Pharm Pract 2014, $3(1): 1-5$.

158. Niriayo YL, Kumela K, Kassa TD, Angamo MT: Drug therapy problems and contributing factors in the management of heart failure patients in Jimma University Specialized Hospital, Southwest Ethiopia. PLoS One 2018, 13(10):e0206120.

159. Kefale B, Degu A, Tegegne GT: Medication-related problems and adverse drug reactions in Ethiopia: A systematic review. Pharmacol Res Perspect 2020, 8(5): $\mathrm{e} 00641$.

160. Kedir H, Berhane Y, Worku A: Khat chewing and restrictive dietary behaviors are associated with anemia among pregnant women in high prevalence rural communities in eastern Ethiopia. PLoS One 2013, 8(11):e78601. 
161. Dendir E, Deyessa N: Substance use and birth weight among mothers attending public hospitals: A case control study. Ethiop J Health Dev 2017, 31(1):27-35.

162. Tesfaye G, Demlew D, Habte F, Molla G, Kifle Y, Gebreegziabher G: The prevalence and associated factors of alcohol use among pregnant women attending antenatal care at public hospitals Addis Ababa, Ethiopia, 2019. BMC psychiatry 2020, 20(337).

163. Tesfay K, Abera M, Wondafrash M, Tesfaye M: Effect of Khat Use During Pregnancy on the Birth Weight of Newborn in Jimma, Ethiopia. Int J Ment Health Addict 2019, 17(6):1432-1441.

164. Jimma University: Jimma University Specialized hospital. http://www.ju.edu.et/jimma-university-specialized-hospital-jush. Accessed on 21 July 2019.

165. Segni H, Ayana D, Jarso H: Prevalence of Hyperemesis Gravidarum and Associated Factors Among Pregnant Women at Jimma University Medical Center, South West Ethiopia: A Cross-Sectional Study. EC Gynaecol 2016, 3(5): 376-387.

166. Siraneh Y, Workneh A: Determinants and Outcome of Safe Second Trimester Medical Abortion at Jimma University Medical Center, Southwest Ethiopia. J Pregnancy 2019, 2019:4513827.

167. Gizaw A, Gemeda T, Yunka T: Perceived Work Environment and Associated Factors among Nurses Working in Jimma University Medical Center, Oromia Regional State South-West Ethiopia. Health Care Curr Rev 2018, 6:227.

168. Kish L: Survey Sampling. New York, London: John Wiley \& Sons, Inc.; 1965.

169. Godlove M: Prevalence of herbal medicine use and associated factors among pregnant women attending antenatal clinic at Mbeya Refferal Hospital in 2010 (M.Sc. Thesis). Muhimbili University of Health and Allied Sciences, Dar es salaam, Tanzania (2011). http://hdl.handle.net/123456789/41. Accessed on 05 October 2019.

170. Mureyi DD, Monera TG, Maponga CC: Prevalence and patterns of prenatal use of traditional medicine among women at selected Harare clinics: a cross-sectional study. BMC Complement Altern Med 2012, 12:164.

171. Mkize GT: An Assessment of Use of Traditional Medicine in Pregnancy \& Associated Factors Among Black South African Women Delivering in Bertha Gxowa Hospital (M.Sc. Thesis). University of the Witwatersrand, Johannesburg, South Africa (2015).http://wiredspace.wits.ac.za/handle/10539/17340?show=full. Accessed on 25 April 2019. 
172. Mothupi MC. Use of herbal medicine during pregnancy among women with access to public healthcare in Nairobi, Kenya: a cross-sectional survey. BMC Compl Alt Med 2014; $14: 432$.

173. Gabbe S, Niebyl J, Galan H, Jauniaux E, Landon M, Simpson J, Driscoll D:

Obstetrics: Normal and Problem Pregnancies, 6th edn. New York: Saunders; 2012.

174. Wells B, Dipro J, Schwinghammer T, Dipro C: Pharmacotherapy Handbook, 9th edn. New York, NY: McGraw Hill; 2015.

175. World Health Organization, United Nations Population Fund, UNICEF: Pregnancy, Childbirth, Postpartum and Newborn Care: A Guide for Essential Practice, 3rd edn. Geneva, Switzerland: World Health Organization; 2015.

176. World Health Organisation: Guidelines for ATC classification and DDD assignment 2016. Oslo, Norway: WHO Collaborating Centre for Drug Statistics Methodology, Norwegian Institute of Public Health; 2017.

177. Mills E, Duguoa J-J, Perri D, Koren G: Herbal medicines in pregnancy and lactation: An evidence-based approach. London ; New York: Taylor \& Francis; 2006.

178. Mills S, Bone K: The Essential Guide to Herbal Safety. St. Louis, Mo: Elsevier Churchill Livingstone; 2005.

179. Gardner Z, McGuffin M: American Herbal Products Association. American herbal products association's botanical safety handbook, 2nd edn. Boca Raton, FL: CRC Press; 2013.

180. Food Medicine and Healthcare Administration and Control Authority (FMHACA): Ethiopian Medicines Formulary 2nd edn. Addis Ababa, Ethiopia: FMHACA; 2013.

181. Kea AZ, Tulloch O, Datiko DG, Theobald S, Kok MC: Exploring barriers to the use of formal maternal health services and priority areas for action in Sidama zone, southern Ethiopia. BMC Pregnancy Childbirth 2018, 18: 96.

182. Federal Democratic Republic of Ethiopia Ministry of health, USAID, JOHNS HOPKINS Center for Communication, JSU: Review of Literature Across Six Health Areas in Ethiopia. Addis Ababa, Ethiopia; 2016.

183. Hailu AD, Mohammed SA: Availability, price, and affordability of WHO priority maternal and child health medicine in public health facilities of Dessie, north-East Ethiopia. BMC Med Inform Decis Mak 2020, 20(1):221.

184. Abrha S, Tadesse E, Atey TM, Molla F, Melkam W, Masresha B, Gashaw S, Wondimu A: Availability and affordability of priority life-saving medicines for under- 
five children in health facilities of Tigray region, northern Ethiopia. BMC Pregnancy Childbirth 2018, 18(1):464-464.

185. Abay SM: Ethiopian herbal medicine practice and the recognition with modern medicine. Pharmacogn Rev 2009, 3(5):44-47.

186. John LJ, Shantakumari N: Herbal Medicines Use During Pregnancy: A Review from the Middle East. Oman Med J 2015, 30(4):229-236.

187. Hall HG, McKenna LG, Griffiths DL: Complementary and alternative medicine for induction of labour. Women Birth 2012, 25(3):142-148.

188. Gokhale S, Sahu A: Pharmacological properties of flaxseed, Linum usitatissimum Linn., as a potential medicinal plant: An overview. World J Pharm Sci 2016, 4(8):207215.

189. European Medicines Agency. Assessment report on Linum usitatissimum L., semen Assessment report on Linum usitatissimum L., Final https://www.ema.europa.eu/en/documents/herbal-report/assessment-report-linumusitatissimum-1-semen_en.pdf. Accessed on 05 November 2020.

190. Gall A, Shenkute Z. Ethiopian Traditional and Herbal Medications and Their Interactions with Conventional Drugs. https://ethnomed.org/clinical/pharmacy/ethiopian-herb-drug-interactions. Accessed on 17 October 2018.

191. Hailemeskel B, Fullas F, Habte A, Al-Matari R, Brewer D: A review of natural remedies commonly used by Ethiopian immigrants in the USA. Curr Res Integr Med 2017, 2(3):31-36.

192. Palla AH, Khan NA, Bashir S, ur-Rehman N, Iqbal J, Gilani A-H: Pharmacological basis for the medicinal use of Linum usitatissimum (Flaxseed) in infectious and noninfectious diarrhea. J Ethnopharmacol 2015, 160:61-68.

193. Kajla P, Sharma A, Sood DR: Flaxseed-a potential functional food source. J Food Sci Technol 2015, 52(4):1857-1871.

194. Holst L, Wright D, Haavik S, Nordeng H: The use and the user of herbal remedies during pregnancy. J Altern Complement Med 2009, 15(7):787-92.

195. Robinson A, McGrail MR: Disclosure of CAM use to medical practitioners: a review of qualitative and quantitative studies. Complement Ther Med 2004, 12(2-3):90-98.

196. Lepik K: Safety of herbal medications in pregnancy. Can Pharm J 1997, 130:29-33.

197. Muhie OA: Antibiotic Use and Resistance Pattern in Ethiopia: Systematic Review and Meta-Analysis. Int J Microbiol 2019, 2019:2489063. 
198. World Health Organization. Iron Deficiency Anaemia; Assessment, Prevention and Control: A guide for programme managers.

https://www.who.int/nutrition/publications/en/ida_assessment_prevention_control.pdf. Accessed on 28 December 2019.

199. Kassa GM, Muche AA, Berhe AK, Fekadu GA: Prevalence and determinants of anemia among pregnant women in Ethiopia; a systematic review and meta-analysis. BMC Hematol 2017, 17:17.

200. Nasir BB, Fentie AM, Adisu MK: Adherence to iron and folic acid supplementation and prevalence of anemia among pregnant women attending antenatal care clinic at Tikur Anbessa Specialized Hospital, Ethiopia. PLoS One 2020, 15(5):e0232625.

201. Gurley BJ, Tonsing-Carter A, Thomas SL, Fifer EK: Clinically Relevant HerbMicronutrient Interactions: When Botanicals, Minerals, and Vitamins Collide. Adv Nutr 2018, 9(4):524S-532S.

202. Imran M, Anjum FM, Butt MS, Sheikh MA: Influence of extrusion processing on tannin reduction and oil loss in flaxseed (Linum usitatissimum L.) meal. J Food Process Preserv 2014, 38(1):622-629.

203. Haidar JA, Pobocik RS: Iron deficiency anemia is not a rare problem among women of reproductive ages in Ethiopia: a community based cross sectional study. BMC Blood Disord 2009, 9:7.

204. Kaluski DN, Ophir E, Amede T: Food security and nutrition - the Ethiopian case for action. Public Health Nutr 2002, 5(3):373-381.

205. Urgessa F, Tsegaye A, Gebrehiwot Y, Birhanu A: Assessment of feto-maternal hemorrhage among rhesus D negative pregnant mothers using the kleihauer-betke test (KBT) and flow cytometry (FCM) in Addis Ababa, Ethiopia. BMC Pregnancy Childbirth 2014, 14:358.

206. Kim YA, Makar RS: Detection of fetomaternal hemorrhage. Am J Hematol 2012, 87(4):417-423.

207. Osaro E, Charles AT: Rh isoimmunization in Sub-Saharan Africa indicates need for universal access to anti-RhD immunoglobulin and effective management of $\mathrm{D}$ negative pregnancies. Int J Womens Health 2010, 2:429-437.

208. Izzo AA: Herb-drug interactions: an overview of the clinical evidence. Fundam Clin Pharmacol 2005, 19(1):1-16.

209. Meshesha SG, Yeshak MY, Gebretekle GB, Tilahun Z, Fenta TG: Concomitant Use of Herbal and Conventional Medicines among Patients with Diabetes Mellitus in Public 
Hospitals of Addis Ababa, Ethiopia: A Cross-Sectional Study. Evid Based Complement Alternat Med 2020, 2020:4871459.

210. Izzo AA, Ernst E: Interactions between herbal medicines and prescribed drugs: an updated systematic review. Drugs 2009, 69(13):1777-98.

211. Alberta Health Services. Beyond the ABCs - Information for Professionals: AlcoholMedication Interactions.

https://www.albertahealthservices.ca/assets/healthinfo/AddictionsSubstanceAbuse/hiasa-beyond-abcs-alcohol-medical-interaction.pdf. Accessed on 29 October 2020.

212. WebMD. Alcohol and Medication Interactions. https://www.webmd.com/mentalhealth/addiction/alcohol-interactions-with-medications\#1. Accessed on 01 December 2020.

213. Johnson BA, Seneviratne C: Chapter 31 - Alcohol-medical drug interactions. In: Handbook of Clinical Neurology. Volume 125, edn. Edited by Sullivan EV, Pfefferbaum A: Elsevier; 2014: 543-559.

214. Baye H, Hymete A: Lead and Cadmium Accumulation in Medicinal Plants Collected from Environmentally Different Sites. Bull Environ Contam Toxicol 2010, 84(2):197201.

215. Bogusz MJ, al Tufail M, Hassan H: How natural are 'natural herbal remedies'? A Saudi perspective. Adverse Drug React Toxicol Rev 2002, 21(4):219-229.

216. Moussally K, Oraichi D, Berard A: Herbal products use during pregnancy: prevalence and predictors. Pharmacoepidemiol Drug Saf 2009, 18(6):454-461.

217. Tuha A, Faris AG, Mohammed SA, Gobezie MY: Self-Medication and Associated Factors Among Pregnant Women Attending Antenatal Care at Kemisie General Hospital, North East Ethiopia. Patient Prefer Adherence 2020, 14:1969-1978.

218. Tesfamariam S, Anand IS, Kaleab G, Berhane S, Woldai B, Habte E, Russom M: Selfmedication with over the counter drugs, prevalence of risky practice and its associated factors in pharmacy outlets of Asmara, Eritrea. BMC Public Health 2019, 19(1):159.

219. Mechessa FD, Dessalegn D, Melaku T: Drug-related problem and its predictors among pediatric patients with infectious diseases admitted to Jimma University Medical Center, Southwest Ethiopia: Prospective observational study. SAGE Open Med 2020, 8:1-9.

220. Ranganathan P, Aggarwal R: Study designs: Part 7 - Systematic reviews. Perspect Clin Res 2020, 11(2):97-100. 
221. Impellizzeri FM, Bizzini M: Systematic review and meta-analysis: A primer. Int J Sports Phys Ther 2012, 7(5):493.

222. Fakeye TO, Adisa R, Musa IE: Attitude and use of herbal medicines among pregnant women in Nigeria. BMC Complement Altern Med 2009, 9:53.

223. Malan DF, Neuba DFR: Traditional practices and medicinal plants use during pregnancy by Anyi-Ndenye women (eastern Côte d'Ivoire). Afr J Reprod Health 2011, 15(1):85-93.

224. Wettermark B, Di Martino M, Elseviers M: Study designs in drug utilization research. In: Drug Utilization Research: Methods and Applications. edn. Edited by Elseviers M, Wettermark B, Almarsdóttir AB, Andersen M, Benko R, Bennie M, Eriksson I, Godman B, Krska J, Poluzzi E et al. Chichester, West Sussex: Hoboken, NJ: John Wiley \& Sons, Ltd; 2016: 13-28.

225. Coggon D, Rose G, Barker D: Epidemiology for the uninitiated, 5th edn. London: BMJ; 2003.

226. Thiese MS: Observational and interventional study design types; an overview. Biochem Med (Zagreb) 2014, 24(2):199-210.

227. Sekaran U, Bougie R: Research methods for business: A skill building approach, 7th edn. Chichester, West Sussex: John Wiley \& Sons Ltd; 2016.

228. Bonita R, Beaglehole R, Kjellström T, World Health Organization: Basic epidemiology, 2nd edn. Geneva: World Health Organization; 2006.

229. Rothman KJ, Greenland S, Lash TL: Modern Epidemiology, 3rd edn. Philadelphia, PA: Lippincott Williams \& Wilkins; 2008.

230. Andrade SE, Gurwitz JH. Pharmacoepidemiology and the Elderly. https://escholarship.umassmed.edu/meyers_pp/265. Accessed on 28 December 2020.

231. Khorsan R, Crawford C: External Validity and Model Validity: A Conceptual Approach for Systematic Review Methodology. Evid Based Complement Alternat Med 2014, 2014:694804.

232. Levin KA: Study design II. Issues of chance, bias, confounding and contamination. Evid Based Dent 2005, 6(4):102-103.

233. Du Prel J-B, Hommel G, Röhrig B, Blettner M: Confidence interval or p-value?: part 4 of a series on evaluation of scientific publications. Dtsch Arztebl Int 2009, 106(19):335-339. 


Review

\title{
The use of medicinal plants by pregnant women in Africa: A systematic review
}

\author{
Seid Mussa Ahmed ${ }^{\mathrm{a}, *}$, Hedvig Nordeng ${ }^{\mathrm{b}}$, Johanne Sundby ${ }^{\mathrm{a}}$, Yesuf Ahmed Aragaw ${ }^{\mathrm{c}}$, \\ Hugo J. de Boer ${ }^{\mathrm{d}}$ \\ a Department of Community Medicine and Global Health, Institute of Health and Society, Faculty of Medicine, University of Oslo, Norway \\ b Pharmacoepidemiology and Drug Safety Research Group, School of Pharmacy, Faculty of Mathematics and Natural Sciences, University of Oslo, Norway \\ ${ }^{\mathrm{c}}$ Department of Obstetrics and Gynaecology, Faculty of Medical Sciences, Jimma Institute of Health, Jimma University, Ethiopia \\ ${ }^{\mathrm{d}}$ Natural History Museum, University of Oslo, Norway
}

\section{A R T I C L E I N F O}

\section{Keywords:}

Africa

Efficacy

Herbal medicine

Medicinal plants

Pregnancy

Safety

\begin{abstract}
A B S T R A C T
Ethnopharmacological relevance: Medicinal plant (MP) use during pregnancy is common in Africa and may have profound effects on both the mother and the developing foetus. A lack of overview complicates monitoring and regulating the use of MPs during pregnancy.

Aim of the study: This systematic review analyses prevalence of use of MPs during pregnancy, regional distribution, types and prevalence, MP properties, potential health risks, and consensus of MPs use, and suggests relevant measures to mitigate negative effects on pregnancy.

Materials and methods: A search was undertaken using a range of scientific databases (Medline, Embase, African Journals OnLine, Google Scholar and Biological Abstracts), non-governmental organisations, various African universities and regulatory websites for original published and unpublished studies that assess and indicate the prevalence of use of MPs during pregnancy in Africa. Additional articles were located by exploring pertinent bibliographies, and contacting experts.

Results: A total of 3659 MP-use studies were found, but only 303 articles received full-text assessment for eligibility and finally only 50 scientific papers were eligible for the systematic review. The prevalence of MP use by pregnant women varied widely from $2 \%$ to $100 \%$. Twenty-eight studies (56\%) specified one or more plant species used as MP during pregnancy. The major reasons for MP use were relief of nausea and vomiting during pregnancy (NVP), stimulation of labour, and facilitation of childbirth. The most commonly cited MP species were Zingiber officinale Roscoe, Allium sativum L. and Cucurbita pepo L. and these were used for relief of NVP, motion sickness and as a nutritional supplement. Route of administration was most commonly oral, and few adverse effects were reported.

Conclusions: The use of MPs among pregnant women in Africa is prevalent, and the most commonly used plant species are not known to have harmful foetal effects during pregnancy. However, many of the MP species are poorly studied and teratogenic effects cannot be ruled out. Collaboration between healthcare providers and traditional practitioners to inform about the safe use of MPs may promote safer pregnancies and better health for mothers and infants.
\end{abstract}

\section{Background}

In Africa, modern health care and medicine is often available only to a limited number of people because either facilities are too expensive or too few facilities are available for too many people. The value of traditional medical knowledge in these countries is high and often confined to a limited number of traditional healers (Pfeiffer and Butz,
2005). Knowledge of medicinal plants (MPs) has often evolved through many generations, a process that has led to many effective remedies, and filtered out many acutely toxic or non-active remedies (de Boer, 2009). At the same time people in many developing countries often suffer poor health, have short life expectancies, and lack effective cures for many ailments (de Boer et al., 2012).

The African continent, generally known for its rich biodiversity, has

\footnotetext{
* Correspondence to: Department of Community Medicine and Global Health, Institute of Health and Society, Faculty of Medicine, University of Oslo, P.O box 1130, Blindern, 0318 OSLO, Norway

E-mail addresses: seidma@studmed.uio.no (S.M. Ahmed), h.m.e.nordeng@farmasi.uio.no (H. Nordeng), johanne.sundby@medisin.uio.no (J. Sundby), yesufahmed47@yahoo.com (Y.A. Aragaw), h.d.boer@nhm.uio.no (H.J. de Boer).
} 


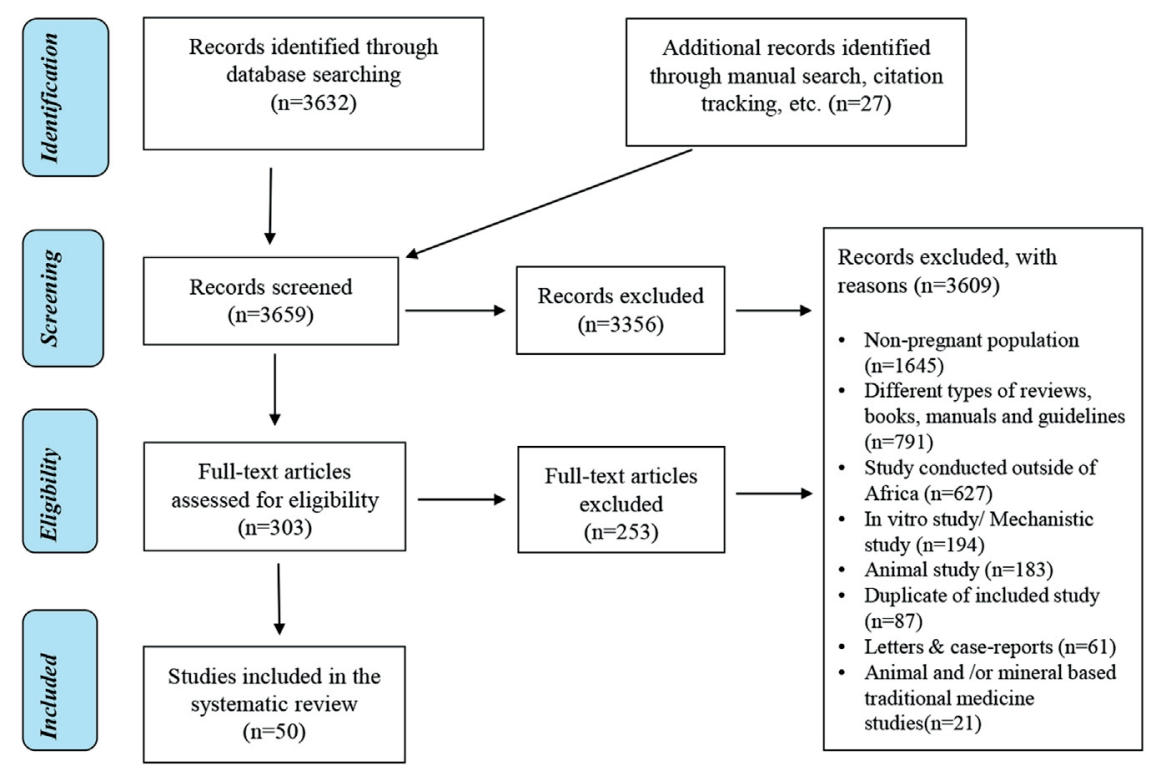

Fig. 1. Identification of 50 studies included in the systematic review.

an estimated total flora of over 70,000 species (Govaerts, 2001). Medicinal plants play a significant role in traditional medicine during pregnancy, childbirth and postpartum care, and include usage for female fertility, menorrhea, birth control, pregnancy, birth, puerperium and lactation (de Boer and Lamxay, 2009; de Boer et al., 2012; Nordeng et al., 2013; Towns and van Andel, 2014; Nergard et al., 2015; Towns and van Andel, 2016). Research focusing on the use of medicinal plants has often inadvertently focused on the realm of knowledge of male traditional healers by relying on traditional patriarchal systems, e.g. male researchers asking the male community leader to identify people knowledgeable in traditional medicine. These biased approaches have missed a wealth of knowledge that is held by women (Pfeiffer and Butz, 2005) and older literature often lists little or no uses of medicinal plants by women (de Boer and Cotingting, 2014).

African women depend on traditional medicine primarily in rural areas for their primary healthcare needs (Kamatenesi-Mugisha and Oryem-Origa, 2007; Towns and van Andel, 2014), but research on the use of herbal medicines for reproductive health matters is still largely limited (Gharoro and Igbafe, 2000; Njamen et al., 2013; Towns and van Andel, 2014). In spite of the specialized knowledge women have on MPs (Camou-Guerrero et al., 2008; Towns and van Andel, 2014), data on the extent of use of MP during pregnancy is especially scarce (Fakeye et al., 2009; Towns and van Andel, 2014). Studies show that medicinal plant use is widespread (Godlove, 2011; van Andel et al., 2014; Laelago et al., 2016), but also highlight data gaps in prevalence, pattern of use, women's perceptions and knowledge about MPs, adverse effects (Kamatenesi-Mugisha and Oryem-Origa, 2007; Godlove, 2011; Kaingu et al., 2011; Malan and Neuba, 2011; Emmanuel et al., 2014; Nergard et al., 2015), spiritual and cultural influences (Bishaw, 1991; Mokgobi, 2014), species identity and consensus of use (Zhang et al., 2014).

The ubiquitous use of MPs during pregnancy in Africa coupled with the piecemeal modernization of healthcare suggests that MPs will continue to play an important role into the near future. The aim of this systematic review is to assess the prevalence and diversity of MP use by women during pregnancy in Africa by looking at published and unpublished studies reporting actual prevalence of MP use during pregnancy. In addition, the review analyses the types and characteristics of MPs used, reasons for use, possible health risks and consensus of use on different medicinal plant species.

\section{Materials and methods}

\subsection{Literature search strategy}

A systematic literature search was conducted from July 2016 to January 2017, and included published scientific literature, unpublished studies, theses and dissertations. The following databases, Medline, Embase, AJOL, Google Scholar and Biological abstracts were searched for articles on MPs use during pregnancy in African countries. For this systematic review, unpublished data were obtained through search for dissertations/theses and reports in African universities websites/libraries (electronic data repositories), medical conference proceedings, regulatory and non-governmental organizations websites and personal contacts. In the review process, the following search terms were included (singular or plural forms when necessary): abortifacient, abortion, adverse, Africa, attendant, birth, botanical, delivery, developing, drug, ethnobotan*, ethnomedicin*, ethnopharma*, expectant, folk, gestating, gravid, healing, herb*, indigenous, labour, medicin*, midwife, mother, native, obstetric, outcome, oxytocic, parturient, parturition, phytomedicine, plant, pregnancy, remedy, sub-Saharan, teratogen*, traditional, treatment, uterotonic, women. Searches were adapted to databases terminology and topic categories. Research articles were also searched by examining bibliographies.

In the literature search process the names of the databases searched, the keywords used and the search results were retained as a 'search diary'. Titles and abstracts of studies considered for retrieval were saved to a Mendeley reference database, along with details of where the reference had been found.

\subsection{Study selection and data extraction}

For a research (article) to be included in the review, it had to assess and indicate the prevalence of use of MPs during pregnancy in an African country, and it should be published or made available within the research period (i.e. up to January 15, 2017). Additionally, studies that reported both the total number of pregnant women that visited healers and those who got MPs treatment were considered. Articles that were excluded were review articles (systematic or literature), those solely concerned with modern medicines, or those where pregnant women were not the study subjects. Furthermore, in vitro studies/mechanistic studies, animal studies, letters, case-reports, books, manuals and guidelines, and those reporting only animal and/or mineral-based 
traditional medicines studies were also excluded. Full publications in print or digital formats were located for all included papers, and analysed for this review (Fig. 1).

The selection of the articles was done in four steps. In the first step, the relevance of the studies was checked based on their title. In the second step, abstracts were evaluated to match to the inclusion criteria. If primary inspection of an abstract of a paper did not give adequate information to make an informed judgment, the full paper was searched in the third step and reviewed by the authors prior to making a decision concerning inclusion in the review. Finally, those that appeared to meet the inclusion criteria were retrieved for extra appraisal by three of the authors (Fig. 1). Data were extracted into spreadsheets according to pre-defined criteria and were summarized in narrative form. The summarized data were compared by the authors and any differences of opinion were resolved by discussion and consultation with the original study. All scientific plant names were checked with The Plant List (www.theplantlist.org).

\subsection{Consensus on MPs plant species}

The Relative Frequency of Citation (RFC) statistic was used to measure consensus on species use between the different studies, and is calculated as $\mathrm{RFC}_{i}=\frac{\mathrm{FC}_{i}}{\mathrm{~N}}$ with $\mathrm{FCi}$ the number of literature sources mentioning species $\mathrm{i}$ and $\mathrm{N}$ the total number of literature sources consulted (Tardío and Pardo-de-Santayana, 2008; Zhang et al., 2014).

\section{Results}

\subsection{Study characteristics}

A total of 3659 publication records were identified, and 303 were assessed and 50 passed the selection criteria for review and data extraction (Fig. 1). Most of the reviewed studies were from West Africa (28 studies), followed by East Africa (13 studies), Southern Africa (6), North Africa (2), and Central Africa (1) (Fig. 2A). An overview of the key characteristics of the 50 included studies is summarized in Table 1. The appraised studies were conducted in the period from 1985 to 2017. Thirty-seven $(74 \%)$ of the fifty studies were executed in the past 7 years. A total of 22,404 African pregnant or lactating women were involved in the respective studies, ranging from 20 in the smallest survey (Kaingu et al., 2011) to 1594 women in the largest study (Yusuff and Omarusehe, 2011). Eighteen studies included a sample of 450 or more participants (Table 1).

MP use is diverse and encompasses many disease prevention, alleviation and treatment methods. Operational definitions of MPs, such as using the WHO definition of traditional medicine (WHO, 2000) was inconsistent in published literature. In this review, 40\% (20 studies) of the reviewed studies defined MPs, whereas the rest did not indicate what was included in the term (for example inclusion of functional foods, prophylactic, strengthening or vitalizing treatments, and dietary proscriptions). Some papers used the WHO definition of traditional medicine (Addo, 2007; Godlove, 2011; Mureyi et al., 2012; Mothupi, 2014; Laelago et al., 2016), but in others the definition varied in one way or the other (Tamuno et al., 2011; Onyiapat, 2014). Some papers included a broad range of traditional medicine therapies in their surveys (Mureyi et al., 2012) whereas others focused on a single specific medicinal plants mixture cf. Isihlambezo (Mabina et al., 1997a, 1997b; Varga and Veale, 1997) (Supplementary Table S1).

Data collection in most studies was based on investigator-informant face-to-face interviews (66\%), and only a minority papers used selfadministered questionnaires (Bello et al., 2011; Abasiubong et al., 2012; Fwacs and Fwacs, 2013; Mugomeri et al., 2015). In approximately a fifth of the reviewed studies (22\%), the authors refer to the use of interview techniques without defining the method. The duration of data collection also varied widely, with some studies collecting data on
MPs use during pregnancy over the course of 18 months (Hillary, 2013; Mothupi, 2014), whereas others were limited to a two week recall period (Kebede et al., 2009). The majority (88\%) were a general crosssectional survey of medicinal plants used by pregnant women, and four employed a combination of cross-sectional surveying with ethnobotanical surveys and/or focused individual discussions with traditional healers and women (Kamatenesi-Mugisha and Oryem-Origa, 2007; Kaingu et al., 2011; Malan and Neuba, 2011; Rasch et al., 2014). The remaining two studies utilized cross-sectional study method along with document review (Opaneye, 1998; Kebede et al., 2009) (Table 1, Supplementary Table S1). Participant sampling methods include random surveys (38\%), representative (consecutive) surveys (34\%), and purposive sampling (12\%) methods, while the remainder did not clearly indicate the sampling method (Supplementary Table S1).

\subsection{Prevalence, diversity and geographic distribution of MPs use}

The reported use of MPs by pregnant women in Africa varies widely from 2\% (Gebremedhin and Gomathi, 2014) to 100\% (Kaingu et al., 2011). The average prevalence rate among the different African regions lies within the range of $30-45 \%$ (Fig. 2B). The highest average prevalence rate was reported from central Africa region (45\%) and the lowest from East Africa (32\%) (Fig. 2B; Table 1). More than half of the studies, $56 \%$, listed the types of MPs used by pregnant women, whereas the other half failed to indicate the kinds of MPs used by expectant women (Table 1, Supplementary Table S1). In aggregation, 28 studies identified a total of 274 different medicinal plants species used in traditional treatment of gestational health ailments/symptom complexes throughout Africa. The distributions of the reported medicinal plants according to African regions are shown in Supplementary Table S2. A higher diversity of medicinal plants (149 plant species) was reported from East Africa followed by West Africa (100 plant species), Southern Africa (32 plant species), and North Africa (14 plant species). Unfortunately, no plant species are indicated in the single study from the Central Africa region (Fig. 2C). Seventeen plant species were reported in more than one region (Supplementary Table S2).

The number of cited plant species varied from study to study, and although 274 medicinal plants were reported to be used by African pregnant women, only 20 species $(8.3 \%)$ along with their prevalence of use were mentioned in two or more articles (Table 2, Supplementary Table S2). The most popularly cited plant species for the treatment of pregnancy disorders and their corresponding RFCs were Zingiber officinale Roscoe (15) (RFC $=0.30)$, Allium sativum L. (12) $(\mathrm{RFC}=0.24)$, Cucurbita pepo L. (7) (RFC $=0.14)$, Vernonia amygdalina Delile and Ricinus communis L. (5 each) (RFC $=0.10)$, Garcinia kola Heckel (4) $(\mathrm{RFC}=0.08)$, Ocimum lamiifolium Hochst. ex Benth., Azadirachta indica A. Juss., Ruta chalepensis L., Aloe vera (L.) Burm.f., and Ocimum gratissimum L. (3 each) $(\mathrm{RFC}=0.06)$. Species with high RFC scores are common and widely cultivated species across Africa, whereas species with low RFC scores are more likely to be restricted in distribution and occur only locally.

The four medicinal plant species used most widely by pregnant women were $Z$. officinale (Ginger), A. sativum (Garlic), C. pepo (Pumpkin) and R. communis (Castor oil). Each of these plants were reported in three regions of Africa. Z. officinale and A. sativum were commonly used in West, East and North Africa (Onyiapat, 2014; Orief et al., 2014; Abeje et al., 2015), whereas C. pepo and R. communis were frequently used in West, East and Southern Africa (Mureyi et al., 2012; Hillary, 2013; Onyiapat, 2014) (Supplementary Table S2).

\subsection{Parts of plant used, methods of preparation and routes of administration}

Various studies reported the use of diverse plant parts as medicinal agents, including root, bark, fruit, bulbs, whole plants, rhizomes, seeds, flowers/inflorescences, and stems, but leaves were the predominant 

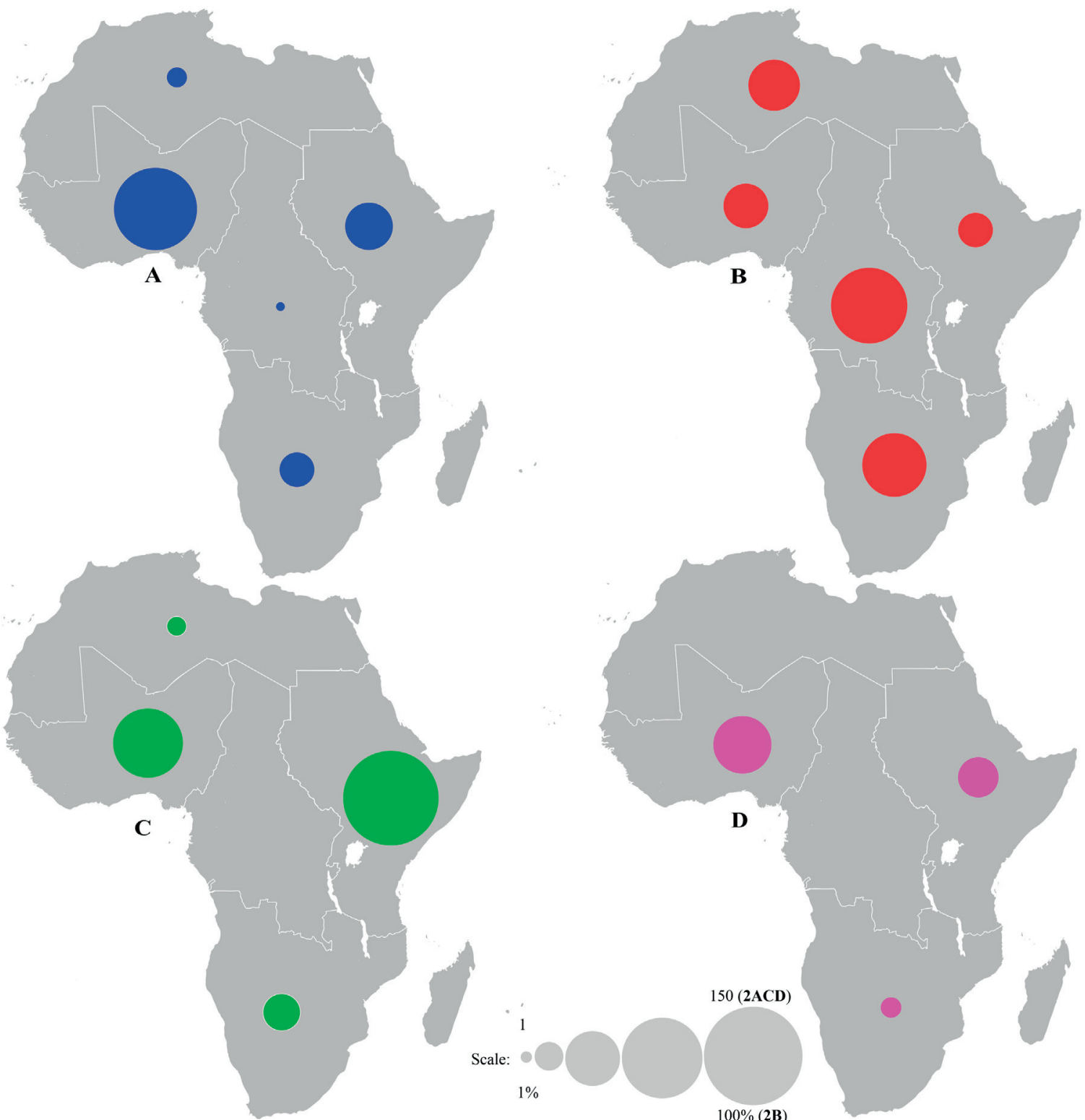

Fig. 2. A. Distribution of number of reviewed studies across African regions. B. Distribution of reported prevalence of MP use across African regions. C. Distribution of reported MP species across African regions. D. Distribution of reported concomitant use of MPs and conventional medicine across African regions.

parts used (Kamatenesi-Mugisha and Oryem-Origa, 2007; Kaingu et al., 2011; Malan and Neuba, 2011; Duru et al., 2016a, 2016b; Hanafy et al., 2016). The commonest methods of preparation/extraction were infusions/tea, maceration, squeezing, chewing, decoction, bathing, evaporating/inhaling as well as ingestion of raw medicinal plant (Varga and Veale, 1997; Kamatenesi-Mugisha and Oryem-Origa, 2007; Bayisa et al., 2014; Nergard et al., 2015). Routes of administration included oral, topical, nasal, intra-vaginal and rectal, but oral was the most frequent route and indicated in 38 (76\%) studies (Varga and Veale, 1997; Kamatenesi-Mugisha and Oryem-Origa, 2007; Kaingu et al., 2011; Malan and Neuba, 2011; Mureyi et al., 2012; Nergard et al., 2015). Surprisingly, the frequency of administration and the dose of the MPs were not indicated in many of the analysed works (Table 1). Concurrent use of oral and rectal routes, and oral, rectal and vaginal together were also reported (Addo, 2007).

\subsection{Reasons for use and sources of information}

Users of MPs during pregnancy had several reasons for consumption of these medicines. Informants in over a quarter of the studies (26\%) reported the use of MPs to alleviate pregnancy associated symptom like nausea, vomiting, etc. (e.g. Kaingu et al., 2011; Aboyeji et al., 2014; Bayisa et al., 2014; Onyiapat, 2014; Duru et al., 2016a; Laelago et al., 2016), whereas others reported improvement of foetal growth (12\%) (e.g. Malan and Neuba, 2011; Adusi-Poku et al., 2015; Mkize, 2015; Mugomeri et al., 2015), stimulation of labour or facilitation of labour and delivery (14\%) (e.g. Mabina et al., 1997b; Kamatenesi-Mugisha and Oryem-Origa, 2007; Omane-Adjekum, 2010; Mureyi et al., 2012; Hanafy et al., 2016), and prevention of premature labour and spontaneous abortions (12\%) (e.g. Kaingu et al., 2011; Malan and Neuba, 2011; Mugomeri et al., 2015). Other uses were specifically postpartum, such as alleviation of postpartum haemorrhage (e.g. KamatenesiMugisha and Oryem-Origa, 2007; Kaingu et al., 2011) and aiding expulsion of the placenta (Kamatenesi-Mugisha and Oryem-Origa, 2007; Kaingu et al., 2011; Olowokere and Olajide, 2013; Adusi-Poku et al., 2015). The South African medicinal plants concoction 'Isihlambezo' was reported to ensure a quick and painless delivery and reduce the placental size (Mabina et al., 1997a, 1997b; Varga and Veale, 1997; Mkize, 


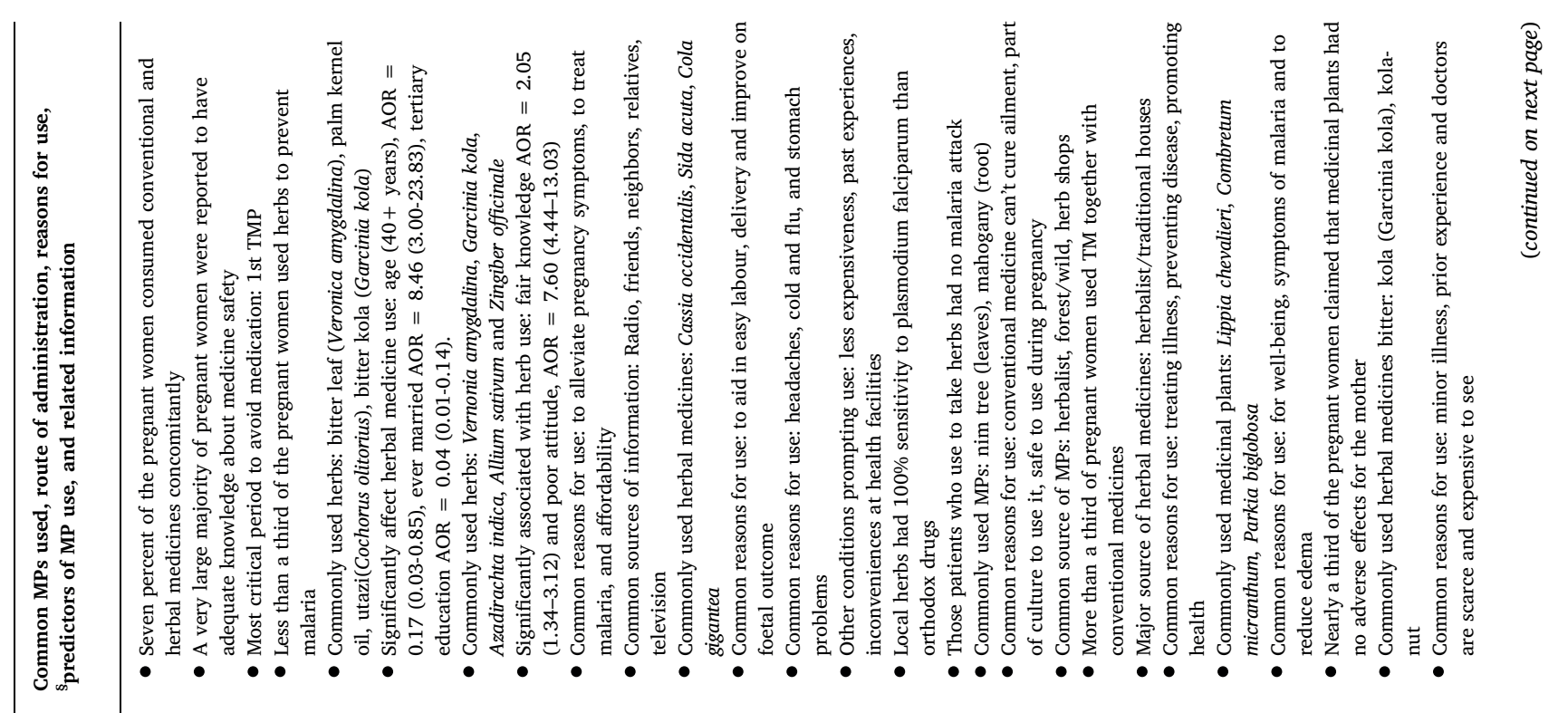

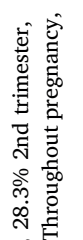

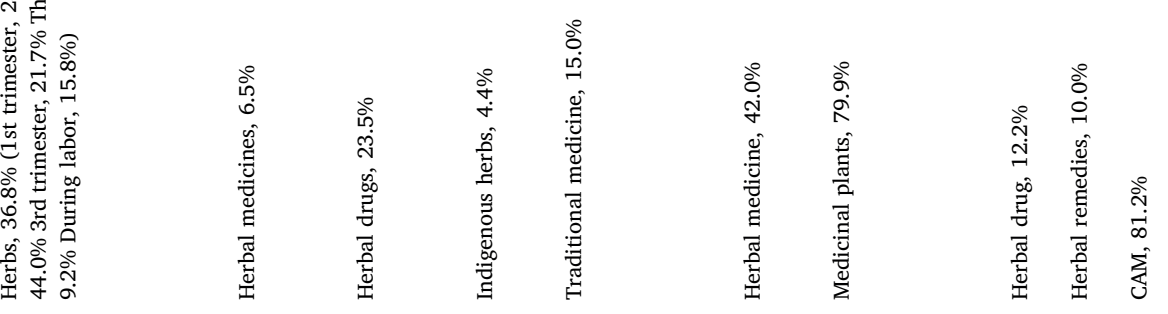

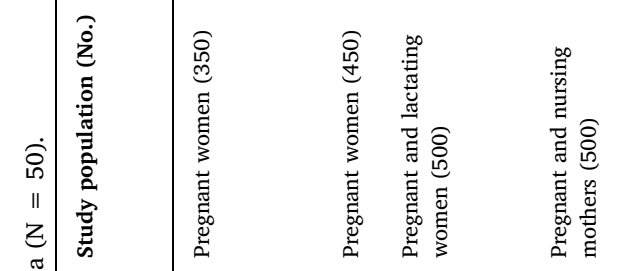
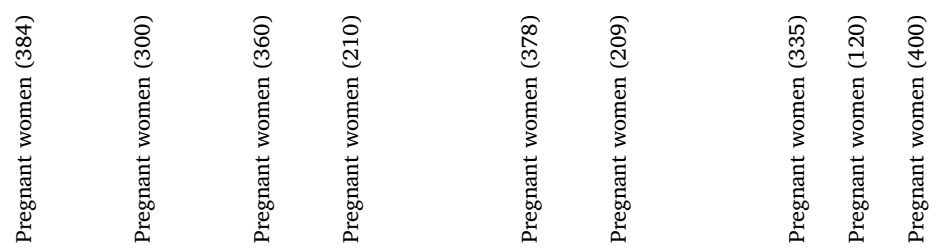

亲

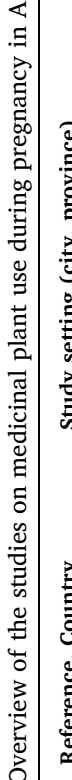

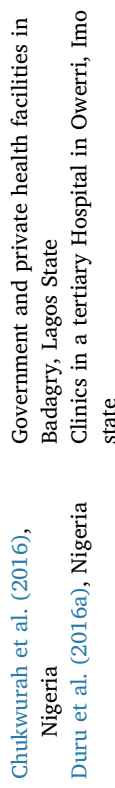

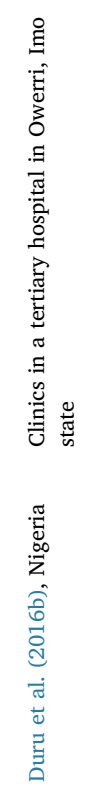

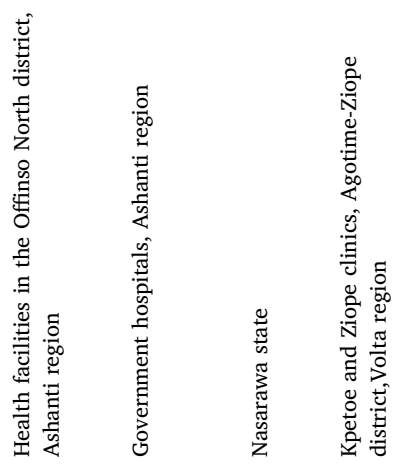

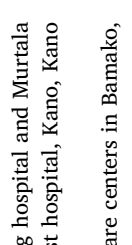

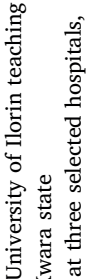

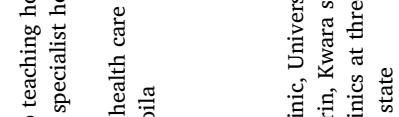

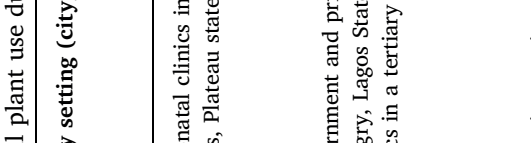

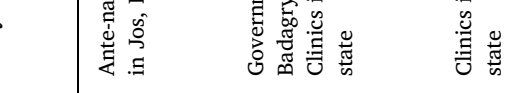

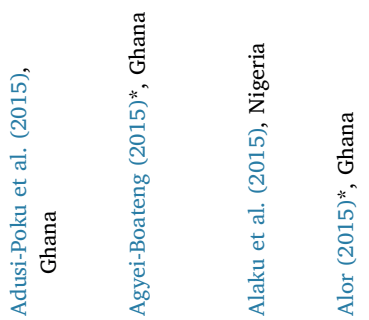

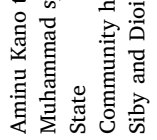

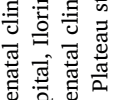

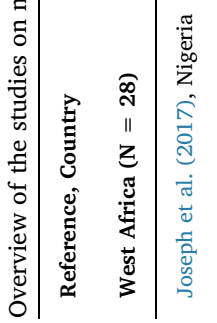

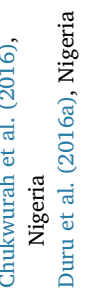
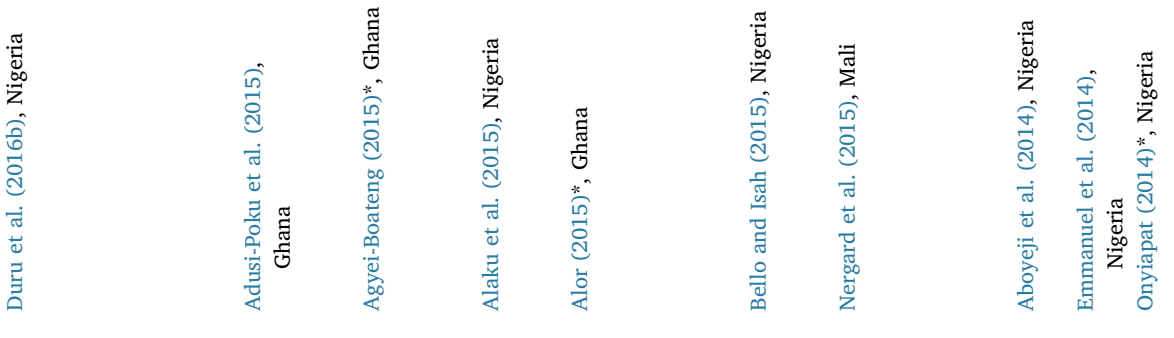


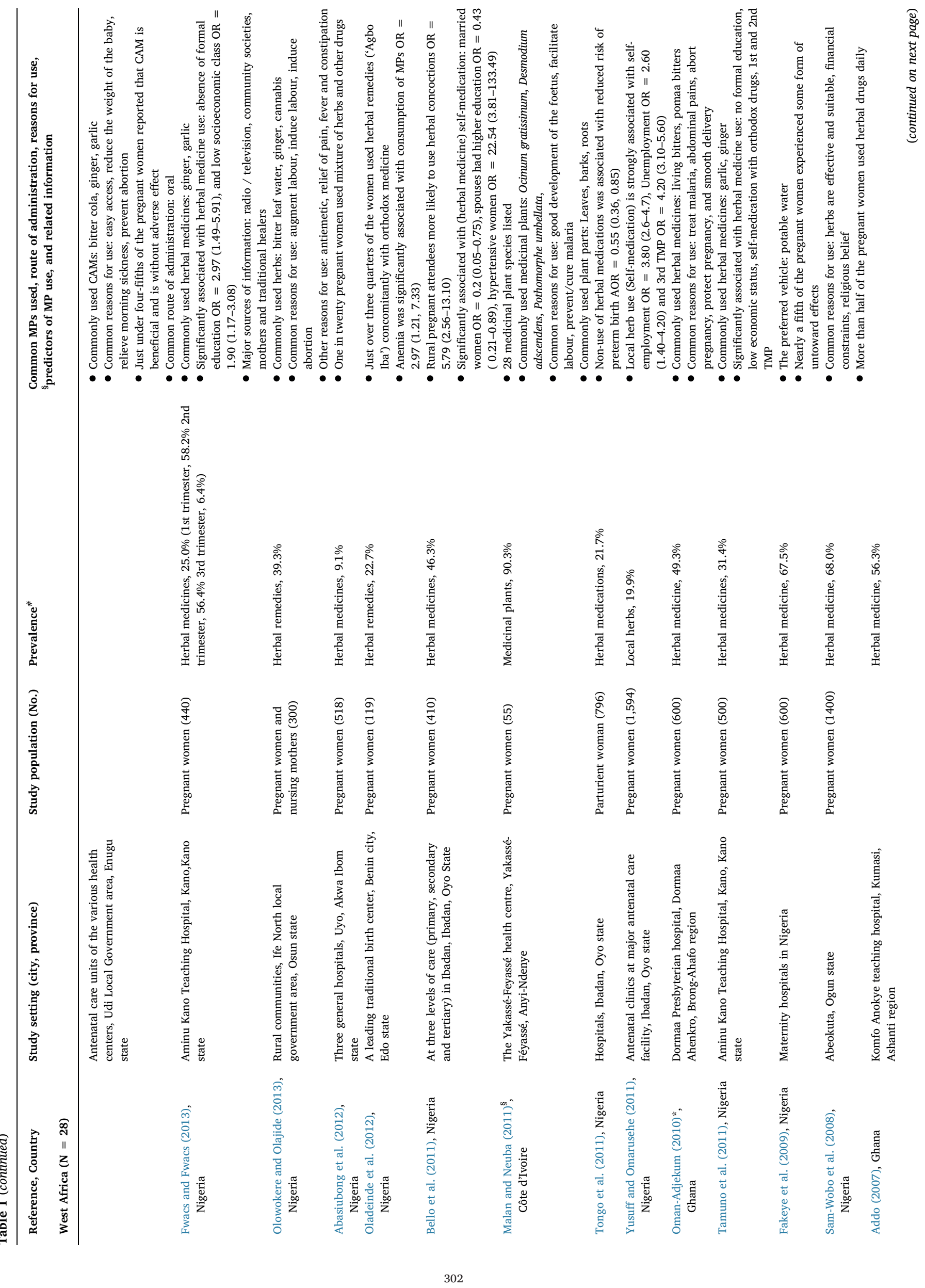




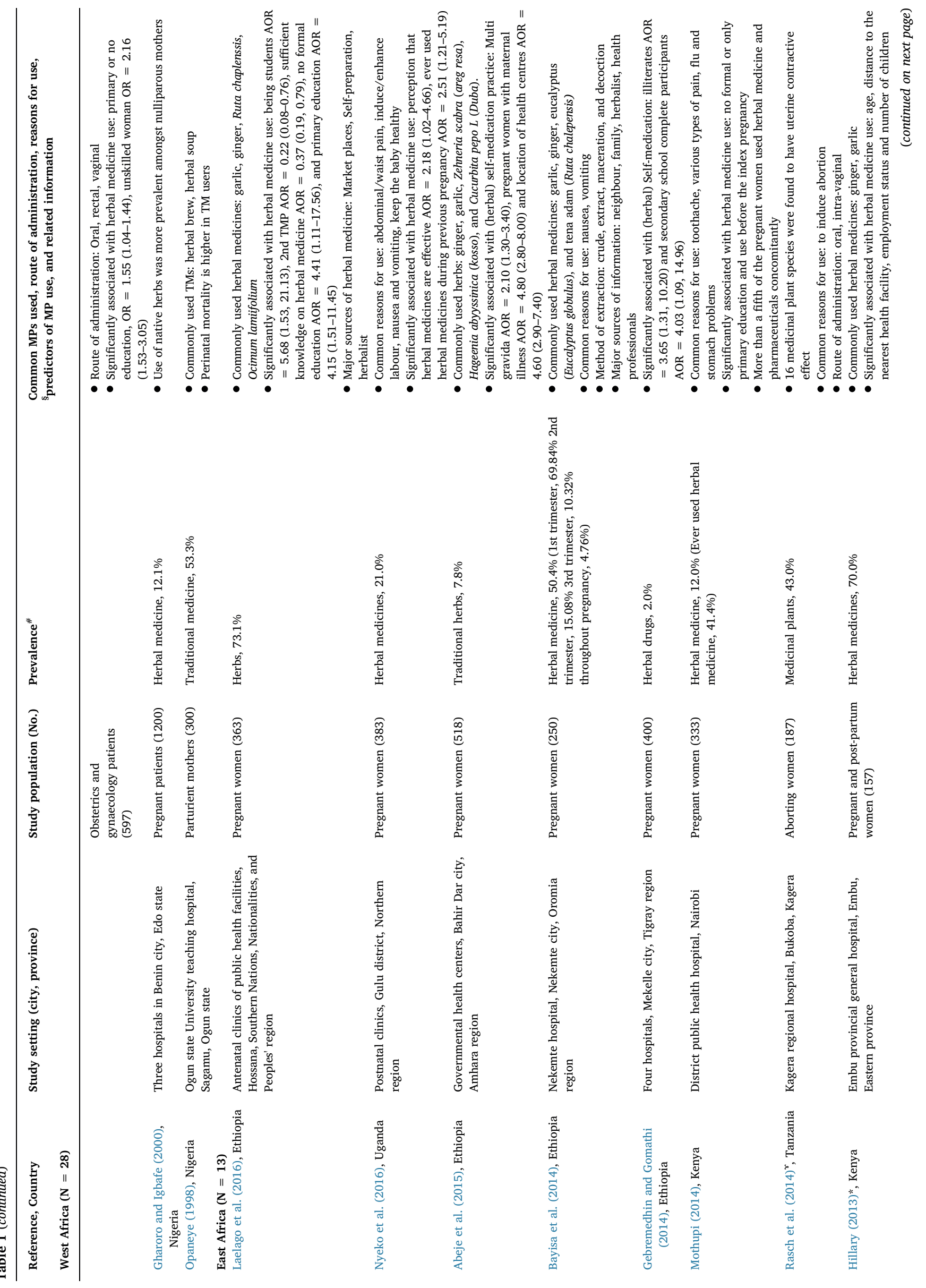




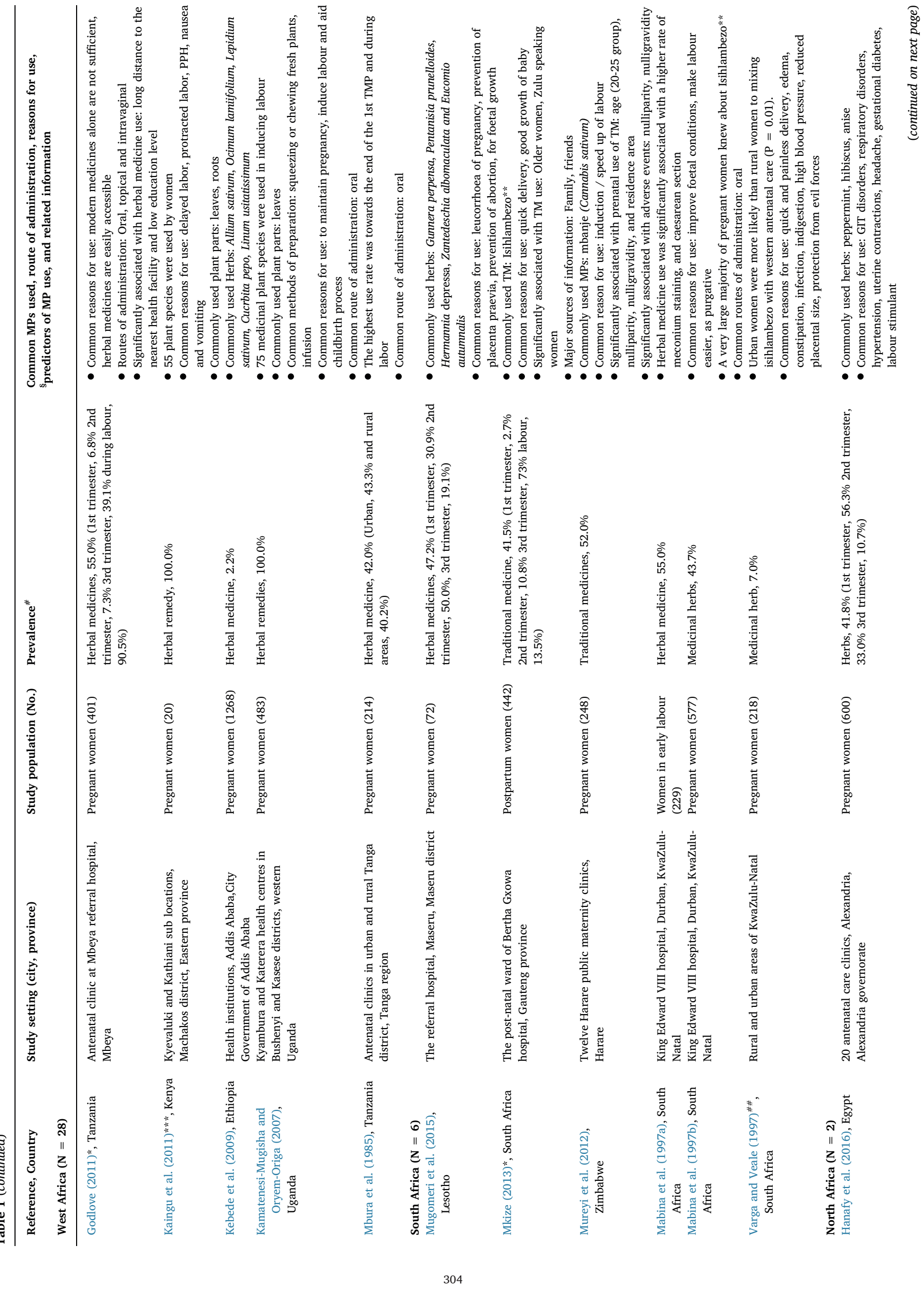


2015) (Tables 1 and 2, Supplementary Table S2).

Nearly half (48\%) of the reviewed materials disclosed the sources from which pregnant women received MP information. The principal sources were family, friends, grandmothers, traditional healers, herbalists, mothers-in-law, parents/relatives and traditional birth attendants (e.g. Orief et al., 2014; Agyei-Boateng, 2015; Mugomeri et al., 2015; Nergard et al., 2015; Nyeko et al., 2016). On the other hand, some expectant women indicated health professionals as a major source of MP information (Bayisa et al., 2014; Agyei-Boateng, 2015; Laelago et al., 2016).

\subsection{Adverse effects and concurrent use of conventional medicine}

Seven studies reported that women perceived MPs to be more effective, work faster and safer to use during pregnancy (Fakeye et al., 2009; Yusuff and Omarusehe, 2011; Hillary, 2013; Nyeko et al., 2016). Contrasting with those results, a few studies reported that respondents avoid using MPs during pregnancy because the MPs are not safe for pregnant women, have adverse/side effects that could be dangerous to their health and the unborn baby and should be avoided or used with caution (Fakeye et al., 2009; Alor, 2015). This appraisal revealed that the majority of the reviewed papers made no mention of possible adverse effects. Only few studies reported some form of adverse effects, and these included excessive uterine contractions, foetal distress and abortions (Godlove, 2011), bleeding and diarrhoea (Onyiapat, 2014), abortion, premature childbirth and foetal death (Mbarambara et al., 2016). The other adverse effects encountered were diarrhoea, dizziness, vomiting, nausea, abdominal pain, weakness and burning sensation (Fakeye et al., 2009; Omane-Adjekum, 2010; Duru et al., 2016a; Laelago et al., 2016).

With regard to concurrent use of MPs with conventional medicine, ten articles reported the concurrent use of MPs with conventional medicine, with prevalence ranging from 2.4\% (Mothupi, 2014) to 77.3\% (Oladeinde et al., 2012). Concurrent use was frequently reported from West Africa (6 studies), followed by East Africa (3 studies) (Fig. 2D). In addition, 22\% of the reviewed studies reported that pregnant women use MPs to treat malaria (Sam-Wobo et al., 2008; Omane-Adjekum, 2010; Malan and Neuba, 2011; Tongo et al., 2011; Oladeinde et al., 2012; Emmanuel et al., 2014; Alaku et al., 2015; Alor, 2015; Nergard et al., 2015; Chukwurah et al., 2016; Duru et al., 2016b).

\subsection{Spiritual and cultural influences}

Religion and witchcraft also played an important role for some of the respondents. Some pregnant women prayed to ancestors to manage pregnancy conditions such as delayed labour; took various MPs to manage protracted labour due to witchcraft or other superstition (Kaingu et al., 2011); drank "church-made" coffee to widen the birth canal and induce labour (Mureyi et al., 2012); drank mixtures of MPs to protection from witches, witchcraft and evil (Abasiubong et al., 2012; Mkize, 2015); or practiced Kombe, a form of Koranic medicine that includes the use of amulets with Koranic inscriptions (Mbura et al., 1985). Furthermore, the South African concoction of medicinal plants 'Isihlambezo' is also claimed to provide spiritual cleansing or protection from evil forces (Varga and Veale, 1997).

\subsection{Predictors of MP use}

Socio-demographic characteristics of MP users and non-users showed interesting differences. In several studies MP use during pregnancy was significantly associated $(\mathrm{P}=<0.05)$ with a lower education level, increasing age, being married, low economic status, lower educational level of spouse, poor pregnancy outcome, MP use experience in previous pregnancy, perception that MPs are effective, large family size, selfemployment, unemployment and rural residence (e.g. Godlove, 2011; Tamuno et al., 2011; Yusuff and Omarusehe, 2011; Fwacs and 


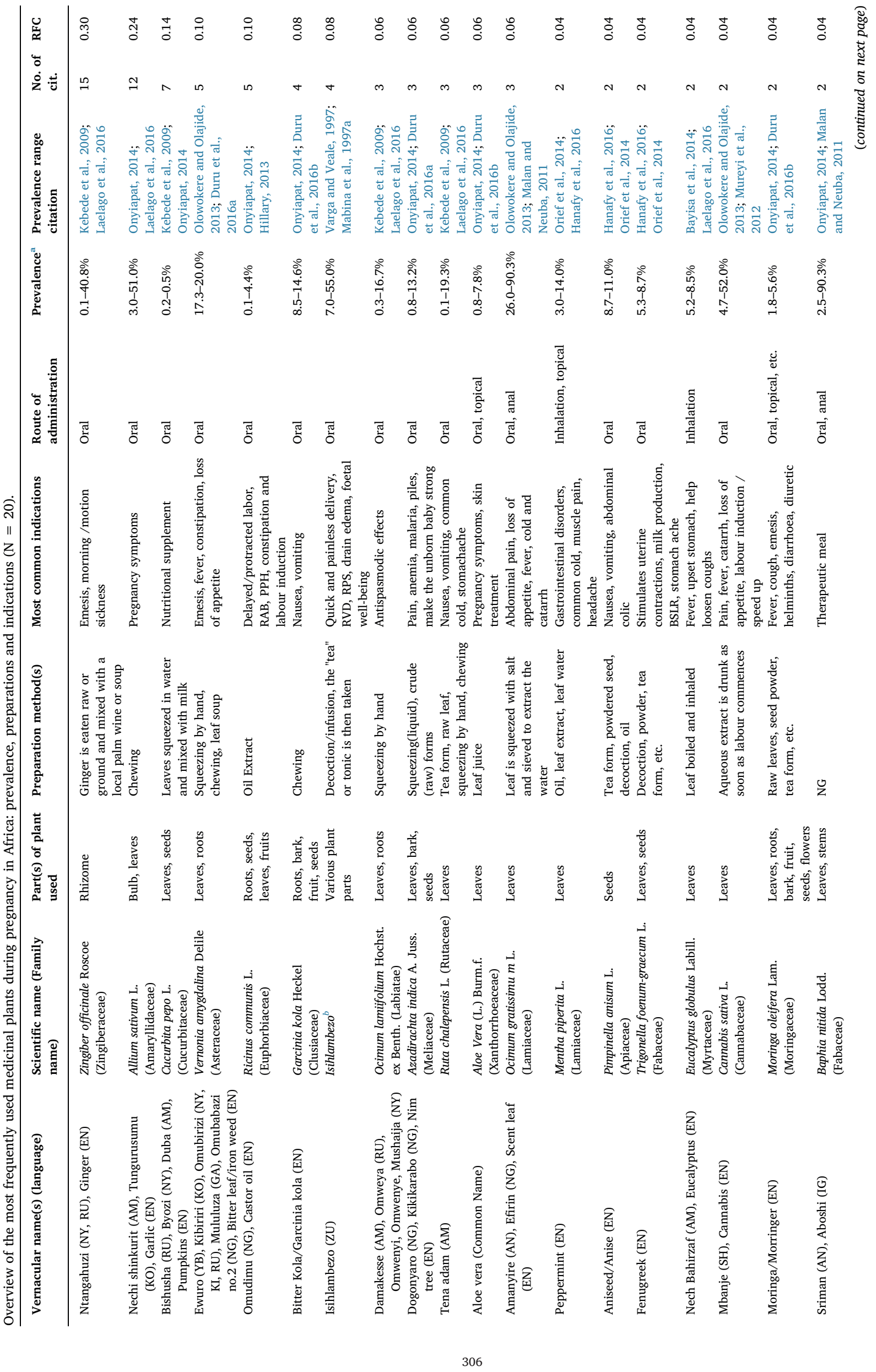


Fwacs, 2013; Hillary, 2013; Olowokere and Olajide, 2013; Mothupi, 2014; Duru et al., 2016a; Laelago et al., 2016; Nyeko et al., 2016). On the contrary, several studies found different results. For instance, Duru et al. (2016a) showed that index pregnancy did not significantly affect the use of MPs during pregnancy. Besides, higher income status was found a strong predictor for MP use during pregnancy (Alor, 2015). Tongo et al. (2011) found that the non-use of MPs preparations was significantly associated with reduced risk of preterm birth. Moreover, Addo (2007) and Mugomeri et al. (2015) disclosed that marital status had no significant association with use of MPs. Anemia was strongly associated with consumption of MP remedies (Oladeinde et al., 2012) (Table 1).

\section{Discussion}

A systematic review of MPs use during pregnancy in the African continent was long overdue, and this comprehensive review increases our understanding of the potential health risks by focusing on MPs utilization pattern and traditions of MPs used during pregnancy among African women. The review covers 13 African countries, and includes 37 papers published in the past seven years, which could suggest an intensification of research focusing on MP use for the treatment and/or prevention of conditions affecting expectant or lactating women. Despite this emerging focus on MPs use during pregnancy, the review has identified a number of gaps in the scientific literature relating to its use.

\subsection{Study characteristics, prevalence of use, diversity and geographic distribution}

Comparison of results across studies is difficult due to differences in methodology and study participants, as well as varying practices between different African countries. Notwithstanding, this review shows that MP use among parturient women in Africa is common, and that a wide range of MPs is used. Existing studies show that the prevalence of MP use among pregnant women varies widely from $2 \%$ to $100 \%$ and this is consistent with findings from other parts of the world. Varying rates of gestational MP usage reported elsewhere were $12-36 \%$ in Australia (Pinn and Pallett, 2002; Forster et al., 2006), 36-40\% in Norway (Nordeng and Havnen, 2004; Nordeng et al., 2011), 7-45\% in the United States (e.g. Gibson et al., 2001; Tsui et al., 2001; Hepner et al., 2002; Glover et al., 2003), 9-96\% in Canada (Westfall, 2003; Moussally et al., 2009), and 39-51\% in Malaysia (Rahman et al., 2007). Similarly, a multinational cross-sectional, internet-based study across Europe, Australia, North and South America involving 9459 women found that $29 \%$ (ranging from $4 \%$ to $69 \%$ ) used phytotherapy in pregnancy (Kennedy et al., 2013). These findings imply that MP use during pregnancy is not only common in Africa as an entrenched part of culture but is also common elsewhere in developed societies where traditions no longer play a strong role. Such differences in the estimates of magnitude of use of MP remedies could be related to variations in study design, data collection methods, cultural features of the studied women, and the definition of MPs used.

Compared to studies done elsewhere, MP use is more prevalent in Africa. This disparity can be attributed to differences in the dominant medical system. In Africa traditional medicine is the primary source of health care for a greater proportion of the population (WHO, 2002; Abdullahi, 2011), leading to a stronger reliance on MPs. Also, the variation depends on socio-cultural contexts, such as study participants, study settings, and health care systems in the different parts of Africa. Considering the differences in number of reviewed studies per region, the computed average prevalence (Fig. 2B) doesn't reliably show regional variability, but it rather indicates where research is lacking. In spite of the variability in MP use rates and number of studies reviewed, the empirical literature does appear to show extensive prevalence rates for MP use amongst expectant women across a number of African 
countries.

The present review showed that African expectant mothers use a wide diversity of MPs, and a total of 274 different plant species in 87 families are reported. On the contrary, a review of studies from the Middle East found that pregnant women used only 18 MPs (John and Shantakumari, 2015). The wide range of medicinal plant species and families reported from Africa could reflect the total species diversity. Even though the lion's share of the analysed studies is from West Africa, over half of the reported medicinal plant species (149 plant species) were from East Africa (Fig. 2A; C). The main reason is that most of the studies from West Africa did not indicate the specific medicinal plant types, signifying that the use of MP during pregnancy is poorly documented and inadequately studied. Additionally, despite having rich floral diversity (Govaerts, 2001), combined with limited access to modern health facilities and high maternal mortality (WHO, 2015; Mbarambara et al., 2016), studies reporting MPs use amongst parturient women are extremely rare in the Central Africa region. This may reflect serious gaps in ethnomedical and/or ethnobotanical studies and documentation of medicinal knowledge and resources in the continent.

\subsection{Reasons for use, safety, efficacy and information sources}

Many of the papers indicated that different medicinal plants are claimed to be used in the management of various pregnancy related illnesses. The top MPs cited in the studies were Zingiber officinale Roscoe, Allium sativum L., Cucurbita pepo L., Ricinus communis L., Vernonia amygdalina Delile and Garcinia kola Heckel (Table 2). Z. officinale (ginger) was the most common species for the treatment of pregnancy induced nausea and vomiting, and reported in 15 studies (Table 2). Previous studies have shown that ginger is commonly used globally by parturient women for nausea and vomiting in pregnancy (NVP) (Maats and Crowther, 2002; Glover et al., 2003; Westfall, 2003; Forster et al., 2006; Holst et al., 2011; Nordeng et al., 2011; Kennedy et al., 2013; de Boer and Cotingting, 2014; John and Shantakumari, 2015). The fact that the same plant species are used by different societies could indicate their effectiveness and is pertinent for prioritizing future studies on efficacy and safety. It should be noted that by looking at consensus we are identifying widely used garden plants as those with highest consensus, but extensive research into these species does support their dual use as nutraceuticals or functional foods. The efficacy of some of the abovementioned MPs in treating nausea and vomiting has been investigated to varying degrees. In a clinical setting in the context of pregnancy, only $Z$. officinale has been studied. A recently published review suggested potential benefits of ginger in reducing nausea symptoms in pregnancy. However, it neither significantly affects vomiting episodes nor poses a risk for negative pregnancy effects in pregnancy (Viljoen et al., 2014). A systematic review critically appraising fourteen randomized controlled trials of herbal therapies in pregnancy found that ginger was the most investigated MP (Dante et al., 2013). It was reported to ameliorate the bouts of nausea and vomiting that women experience during parturition better than placebos. A prior systematic review of randomized clinical trials of ginger suggested that ginger was more effective than placebos and about as effective as metoclopramide for nausea and vomiting (Ernst and Pittler, 2000).

The use of MPs is increasing throughout Africa and globally, but there is scant research on the safety of medicinal plants especially during pregnancy (Adusi-Poku et al., 2015;). Even though all the fifty reviewed studies reported the use of medicinal plants during pregnancy, only a little over $10 \%$ found that the informants perceived phytomedicines to be more effective and free of adverse effects. Concerning the safety of MPs during pregnancy, reliable data are currently lacking and often contradictory. A review that evaluated the safety of ginger for NVP revealed it did not have harmful maternal or neonatal effects (Boltman-Binkowski, 2016). Furthermore, a cohort study from Norway showed that it was not associated with a higher-risk of birth defects, premature birth, perinatal mortality, or low birth weight (Heitmann et al., 2013). However, many of the MPs used in parturition are understudied (Black and Hill, 2003) and thus, contrary to these reports, concerns exist as to the safety of these medicinal plants. For example, Z. officinale, is reported to have serious adverse effects including mutation of the foetus, abortifacient, emmenagogue effects and increased risk for bleeding (Fischer-Rasmussen et al., 1991; Ernst, 2002; Wen et al., 2008). In addition, prenatal exposure to ginger is associated with increased uterine activity, foetal loss, and inhibition of platelet aggregation (Guh et al., 1995; Spolarich and Andrews, 2007). Also, there is no adequate information about the hazards of its long-term usage in pregnancy. Thus, cautious use is recommended and further research is needed to verify the clinical safety of ginger.

In this study, in line with research and reviews from other corners of the world (Ernst, 2002; Westfall, 2003), R. communis (Castor oil) was claimed to be used to aid labour and relief of constipation (Table 2). A prospective evaluation by Garry et al. (2000) indicated castor oil to be effective for inducing labour. However, another study demonstrated castor oil to have no effect on the time of birth (Boel et al., 2009). Its most common reported side effect was nausea. Nevertheless, there is paucity of research data on neonatal mortality or morbidity that may result from castor oil ingestion (Boel et al., 2009; Dante et al., 2014). Furthermore, there is no compelling evidence of efficacy for its use in initiating labour (Ernst, 2002).

The other group of MPs frequently used by pregnant women were $A$. sativum, C. pepo, and M. piperita (Table 2). A. sativum is used to alleviate pregnancy symptoms including NVP. Studies from other places have shown that it enhances the immune system of parturients (Charlson and McFerren, 2007) and has antifungal properties (Westfall, 2003). Likewise, M. piperita is used for handling of gastrointestinal disorders, common cold, muscle pain, and headache. A clinical trial showed that compared to placebo peppermint oil was found to be more effective in the treatment of post-operative nausea (Tate, 1997). However, for all the three plants, there is scarcity of clinical experiments about their safety and efficacy in NVP (McKay and Blumberg, 2006; Charlson and McFerren, 2007; Wills and Forster, 2008; Adnan et al., 2017). Many of these MPs are generally harmless in small doses; however, they can cause serious effects when used in large amounts (John and Shantakumari, 2015). The fact that these phytomedicines are among those widely utilized in Africa is apprehensive.

Anticipating mothers will seek knowledge when confronted with ailments and discomfort during pregnancy, and this can come from many sources (Westfall, 2003). In the current review, families, friends, traditional healers, and traditional birth attendants were indicated as the main sources of information about MPs. Likewise, family and friends were the prime sources of information about medicinal plants for Arabian (John and Shantakumari, 2015), Canadian (Hollyer et al., 2002; Westfall, 2003), the US (Tsui et al., 2001), UK (Holst et al., 2009) and Norwegian (Nordeng and Havnen, 2004) parturient population. As pregnancy is a time of particular vulnerability, it is worrying that mothers do not look for advice from appropriate healthcare professionals. Family members and confidants may simply not have quality and accurate information to provide counselling to pregnant women (Nordeng et al., 2013). This is a solid indication that health care providers, especially those working at the primary healthcare level, should ensure that they have proper comprehension to advise women in a culturallysensitive context (Skouteris et al., 2008; Hall et al., 2011). Considering the high popularity of MPs in pregnancy in Africa, care providers should be more diligent in asking questions about the use of MPs and document it in the patient medical record. Additionally, pregnant women should be informed that 'natural' can be equated with 'safe' is not essentially always the case. In many teaching curricula in Africa traditional medicine is currently overlooked, and this should be addressed to provide better and more balanced advice to expecting mothers. 


\subsection{Potentially unsafe MPs and concurrent use of conventional medicine}

In seven of the reviewed studies, women reported one or more adverse effects. Similar to this finding, mild to severe adverse events were reported in reviews conducted in other areas (Posadzki et al., 2013). Many medicinal plants that are consumed by African expectant women were reported to have potentially toxic effects for the mother and the foetus (Supplementary Table S2). For example, Ruta chalepensis L. contains furanocoumarins that are embryotoxic, and cause implantation failure and abortion (de Sa et al., 2000; Ciganda and Laborde, 2003). Steenkamp (2003) in her review revealed that some plants used during pregnancy resulted in adverse consequences: Callilepis laureola DC. can cause hypoglycemic and strychnine-like symptoms, Clivia miniata (Lindl.) Bosse can cause paralysis and collapse effects, and Rhoicissus tridentata (L.f.) Wild \& R.B.Drumm. can cause paralysis of the central nervous system and subsequent respiratory arrest. Another review identified many toxic plants such as Eucomis autumnalis (Mill.) Chitt. (abdominal pain, diarrhoea, renal failure), Gnidia kraussiana Meisn. (severe gastrointestinal irritation, death of stock), and Scadoxus puniceus (L.) Friis \& Nordal (dizziness, visual disturbances, CNS excitation or depression) (Veale et al., 1992). In his review, Ernst (2002) identified several other MPs with potential adverse effects. In view of these risks from poisonous plants and added chemicals, the indiscriminate use of MPs by pregnant women is a major concern. Until convincing scientific evidence emerges, it is safest to consider all MPs contraindicated in pregnancy (Ernst, 2002).

With respect to simultaneous use of MPs and pharmaceuticals, ten of the reviewed studies indicated that women took both together, and this practice is more prevalent in Western Africa (Sam-Wobo et al., 2008; Omane-Adjekum, 2010; Yusuff and Omarusehe, 2011; Abasiubong et al., 2012; Oladeinde et al., 2012; Joseph et al., 2017) (Fig. 2D). In addition to producing adverse effects, in concomitant administration there is a threat of interaction between MPs and other medications, potentially making them less active or less safe. For instance, A. sativum, the second most commonly cited plant in this systematic review has been described to have strong potential drug interactions with antiplatelet and thyroid replacement therapy medications (Gall and Shenkute, 2009). Additionally, it has been shown to lower blood pressure and should not be taken simultaneously with antihypertensive medications (Auer et al., 1990). An interaction with the anticoagulant warfarin has also been reported (Izzo, 2012). Similarly, simultaneous consumption of $C$. pepo with warfarin was reported to have increased anticoagulant effects (Yue and Jansson, 2001). Thus, concurrent use warrants continuous consideration on the part of both expectant mothers and caregivers. In addition, health workers ought to remain watchful for potential interactions between MPs and orthodox medicines.

Another form of concomitant use, or failure to seek adequate treatment, is the management of malaria using MPs. One in five papers disclosed that parturient women use MPs to prevent or treat malaria. A recent review found that the principal reason for MP use was the perception of lower severity of malaria symptoms (Suswardany et al., 2015). Studies from Africa have reported various misconceptions concerning the causes of malaria, such as the belief that it can be caused by cloudy weather conditions, evil spirits, starvation, 'mitch', and too much workload (Legesse et al., 2007; Mussa and Gedif, 2011a). These misconceptions, coupled with others, could contribute to delays in seeking adequate treatment. Owing to these misbeliefs, self-medication following self-diagnosis of malaria based on presumptive symptoms is commonplace in Africa (Mussa and Gedif, 2011b; Towns and van Andel, 2014). However, due to impaired immunity and a diminution of acquired immunity, malaria infection during parturition has a considerable risk for the mother, her unborn infant and the newborn (WHO, 2014). It can lead to anemia, which increases the risk for maternal and infant mortality and growth problems for babies (WHO, 2014; Suswardany et al., 2015). Hence, women should be encouraged to seek effective treatment from health facilities.

\subsection{Parts of plant used, methods of preparation, and routes of administration}

Steenkamp (2003) in her review of MPs used by South African women for gynaecological complaints found that roots were the dominant plant parts used to prepare remedies. Besides, a review by Alebie et al. (2017) reported that roots were the second most plant parts used as antimalarial agents. Contrary to those findings, in this study covering the entire African continent we found that leaves are the most commonly used plant parts to treat various pregnancy induced health problems. The frequent use of roots for medical purposes is problematic as sustainable harvesting of roots is often difficult and in some cases threatens popular species (Alebie et al., 2017). To avoid the loss of valuable medicinal plant species, use of leaves is much more sustainable than roots, tubers and rhizomes (Cordell, 2015).

Dosage forms and preparation methods of MPs are extremely important. Parturient women in Africa prepare MP formulations through various methods. The most frequently cited independent MP preparation approaches were decoctions, infusions, macerations, concoction, crude extracts (by squeezing) or chewing/eating plants (Table 1). The diversity of methods of preparation by African women may be due to the fact that different plant parts need different methods to extract the active plant ingredients. Studies from other parts of the world have also reported that decoction and infusion/maceration were the most frequently used methods of MP remedy preparation (Rodrigues, 2007; de Boer and Lamxay, 2009).

Although various routes are employed for the administration of MP preparations, this review found that oral intake was the predominant route (Table 2). This is consistent with other studies that reported oral as a popular route of administration (Steenkamp, 2003; Alebie et al., 2017). Oral administration is an effective, non-invasive and convenient method of administration (Verma et al., 2010). Moreover, most of the products of decoction, infusion, and maceration are liquids, and these are easily taken orally. These might be the basic reasons for its extensive employment by the African expectant mothers. Because it is convenient, cheap and amenable for a variety of dosage forms, all the top ten MPs identified in this review were exclusively administered orally (Table 2).

\subsection{Some African MP concoctions}

In African MP practice pregnant women used different treatment approaches not reported somewhere else. Some of the main indigenous approaches reported in the literature include 'isihlambezo', and 'therapeutic meal'. The first, 'isihlambezo', is a mixture of MPs used by women in South Africa (Mabina et al., 1997a, 1997b; Varga and Veale, 1997; Mkize, 2015) as a preventative health tonic in pregnancy that is rich in essential minerals and vitamins. It is a mixture of around ten different plant species (Mabina et al., 1997a, 1997b; Varga and Veale, 1997; Mkize, 2015), and the mixture of species may produce additive, synergistic, or antagonistic effects. Varga and Veale (1997) studied this concoction of MPs extensively and have shown that many of the constituent species have in-vivo uterotonic properties. As a consequence, if used inappropriately it may lead to premature delivery. Mixed MPs remedies are also likely to include plant species with adverse effects, and Veale et al. (1992) and Mathee et al. (2014) both reported that several 'isihlambezo' species were associated with detrimental pregnancy outcomes, such as death due to hepatorenal failure, low birth weight, meconium staining of the amniotic fluid and foetal distress. The constituent plant species of 'isihlambezo' have yet to be scientifically evaluated and investigated whether they possess the claimed medicinal values and reported adverse effects.

The second is 'therapeutic meal'. It is prepared from both animal and plant products, and consumed orally as meal or drunk as a liquid 
preparation. African oil palm (Elaeis guineensis Jacq.) tree fruits are mixed with two or three plantains and cooked. Second, these cooked particular ingredients are crushed, with a little water, in a mortar and filtered. The filtrate is collected and cooked again with the common ingredients used for the preparation of sauces such as peppers, tomatoes, etc. Additionally, smoked freshwater catfish (Chrysichthys nigrodigitatus), or the dried legs of 'blue duiker' (Cephalophus montícola) are added to make this special sauce. The meal is consumed by pregnant women, from the end of the second trimester to delivery. Other medicinal plants such as Ricinodendron heudelotii (Baill.) Heckel, Dracaena mannii Baker, Piper umbellatum L., Nephrolepis biserrata (Sw.) Schott, Solanecio biafrae (Oliv. \& Hiern) C.Jeffrey, Ocimum gratissimum L., or Microdesmis keayana J. Léonard can be ingredients of this special recipe. As divulged by Malan and Neuba (2011), the food is believed to make the children mainly strong and belligerent: 'After feeding, it is formally forbidden to wash the hands, rather it is wiped on the belly, from top to bottom, towards the loins, by saying the wish to have a beautiful baby and an easy labour.' The meal is beneficial for the pregnant women for four reasons. Firstly, it is a rich source of calories, oligonutrients, lipids, and proteins essential to foetal growth. Secondly, it prevents women from suffering from malnutrition, avoiding foetal low birth weight and birth defects as well (Malan and Neuba, 2011). In addition to its benefit for the foetus, many of the component plant species of 'therapeutic meal' were also reported to strengthen women during pregnancy and facilitate delivery (Towns and van Andel, 2016). Finally, the closing ritual, which ends the meal, is a substantial psychological stimulant (Malan and Neuba, 2011).

\subsection{Spiritual and cultural influences}

Religion and spirituality are often interwoven with traditional healing in Africa, and many people turn to both in case of ailments (Mokgobi, 2014). In addition to their perceived pharmacological values, MPs are claimed to provide spiritual cleansing or protection from evil spirits. For example, Kaingu et al. (2011) indicated many pregnant women relied on prayers to ancestors (called by various names depending on one's ethnic origins) to handle delayed labour and they believed protracted labour resulted from either owing to witchcraft or the expectant women having offended their mother-in-law. In Africa the risk of maternal death is greatest during labour and the postpartum period (WHO, 1991; USAID, 2012; Say et al., 2014), and the major statistical causes of mortality are haemorrhage, sepsis/infections, and hypertensive disorders (Khan et al., 2006; Kinney et al., 2010; Haeri and Dildy, 2012; Tort et al., 2015). Culturally-sensitive introduction of modern healthcare practices have the potential to minimize barriers, enhances the utilization of skilled maternal health care services, improves health outcomes, and increases customer satisfaction with services (Anderson et al., 2003; USAID, 2012). In addition to treating diseases, African traditional medicine is also attentive to the protection and promotion of spiritual, social, psychological and physical wellbeing (Bishaw, 1991). The current finding explicitly indicates parturient women use religious rituals during labour and postpartum. Relying on religious beliefs instead of seeking effective healthcare is arguably more dangerous than putting faith in MPs as the former will definitely not be more effective than a placebo. Healthcare professionals should be aware of existing religious or cultural beliefs that may influence healthseeking behaviour of pregnant women, and targeted public education campaigns and collaborations with community and religious leaders can help prevent unnecessary mortality.

\subsection{Predictors of MP use}

Some of the factors significantly associated $(\mathrm{P}=<0.05)$ with MP intake found here are different from previously reported studies. Generally, lower levels of education, marital status, rural residence, low socioeconomic class, unemployment, long distance to the nearest health facility, perception that MPs are effective, experience from previous pregnancy, self-employment, and large family size were found significantly associated with higher use of MPs during pregnancy. Contrary to our review findings, having higher education and better income levels, were indicated as predictors of MP usage in other places (Hall et al., 2011; Frawley et al., 2013; Kennedy et al., 2013). A study done among Australian pregnant women reported that MP users were more likely to be nulliparous (Forster et al., 2006). However, our review showed that large family size and MP treatment experience in previous pregnancy correlated with MPs use during pregnancy. Moreover, our review found that pregnant attendees of rural centers to use MPs significantly more and more frequently than their urban counterparts. In Africa, access to primary healthcare facilities is difficult. People in the rural area need to walk long distances to see health professionals (Makita-Ikouaya et al., 2010). On the other hand, traditional healers and medicines are accessible within a short walking distance. Traditional remedies are often less costly, delivered either free or with a relatively low cost. Besides, traditional medicines are thought to be safe and efficacious, follow holistic perceptions of disease and other human problems (Bishaw, 1991). Furthermore, the payment modality of healers is usually based on the ability of the patient (Gedif and Hahn, 2002) making them affordable and a reliable choice for large family size, the poor and unemployed women.

\subsection{Strengths and limitations}

This systematic review presents the first comprehensive review of studies on the use of MPs during pregnancy in the African continent. Furthermore, the review provides a valuable overview for health experts, researchers and policymakers. However, like any other review, this one has its own inherent limitations that must be kept in mind when interpreting the evidence. All articles are prone to publication bias, and thus any such bias may have been transferred to our review. It is important to note that the study approach does not yield an exhaustive review of literature reporting MPs for women's healthcare or pregnancy. The focus here was only on studies reporting actual prevalence of use of MPs during pregnancy, and this approach excluded a large body of qualitative and quantitative ethnobotanical literature reporting specific plant species for use during pregnancy and postpartum recovery without citing actual use (cf. van Andel et al., 2014). Additionally, the prevalence presented may not signify the real prevalence due to the differences in the reviewed studies. On the whole, this review provides useful and systematically compiled data for practitioners, policymakers and researchers interested in understanding and addressing the challenges of managing and treating ailments in pregnancy in Africa.

\subsection{Identified gaps}

Despite emerging focus upon MP use in the course of pregnancy throughout Africa, the review has identified several gaps in the scientific literature relating to MP use. Our review indicates a general lack of research focus on the extent of MP use in Africa during pregnancy. For example, only one study is retrieved from the Central Africa region. It also indicates existence of critical research evidence gaps with regard to potential medicinal plant toxicities, possible medicinal plants-pharmaceutical drug interactions and adverse effects. The detailed reasons and determinants for greater reliance on MP among African women also need further exploration. Besides, there is little information regarding the types of African MPs and users' profiles.

\section{Conclusions and recommendation}

The prevalence of MP use by African pregnant women was found to be high. Z. officinale, A. sativum and C. pepo were the most commonly cited MP species, and these commonly used plant species are not known 
to have detrimental effects during pregnancy. However, many of the species are poorly studied and teratogenic effects cannot be ruled out. The review also reveals the use of several species that are known to have uterine contracting and harmful effects. Our review indicates that MP use studies during pregnancy across Africa are limited. Although African pregnant women use different medicinal plants for their healthcare, there is a dearth of information regarding their ethnopharmaceutical, ethnobotanical and ethno-medical characteristics, determinants and reasons of use. The review also showed that it is not uncommon for pregnant women to take modern and MPs concurrently, hence research focusing on its extent, its untoward effects and management strategies are timely for investigation. Moreover, though several African MPs used for the treatment of pregnant women's disorders were claimed to be safe and effective, in most cases their safety and efficacy is not compiled and scientifically established. Thus, further evaluation and classification of the safety of MPs used among African pregnant women is important. More research on MP use during pregnancy is required to fully inform all stakeholders engaged in preventing maternal mortality and treating morbidity. Primary healthcare providers should be the first-line of information for pregnant women, but there is also a need for training on the potential benefits and dangers of MP use during pregnancy to enable these professionals to provide culturally sensitive and objective advice. Further research and collaboration with traditional practitioners on the safe use of MPs in pregnancy may promote safer pregnancies and better health outcomes for women and their unborn foetus in Africa.

\section{Acknowledgements}

Not applicable.

\section{Funding statement}

This review did not receive any specific grant from funding agencies in the public, commercial, or not-for-profit sectors.

\section{Authors' contributions}

Review concept and design: SMA and HN; Literature search: SMA, HN and HDB; Analysis and interpretation of data: SMA and HDB; Drafting of the manuscript: SMA, HN and HDB. Critical revision of the manuscript for important intellectual content: SMA, HN, JS, YAA, and HDB. All authors have read and approved the final version.

\section{Definition of terms:}

Adverse drug reaction. A response to a drug which is noxious and unintended, and which occurs at doses normally used in man for the prophylaxis, diagnosis, or therapy of disease, or for the modification of physiological function (World Health Organization).

Agbo Iba. A multi-medicinal plant extract and the component species are Cajanus cajan (L.) Millsp., Euphorbia lateriflora Schumach ., Mangifera indica L., Cymbopogon giganteus Chiov. , Sarcocephalus latifolius (Sm.) E.A.Bruce, and Uvaria chamae P.Beauv.

Church-made coffee. A collection of concoctions and beverages with undisclosed ingredients given to women to fend off 'malicious spirits' or to widen the birth canal to make childbirth safer and easier. This is common among certain religious beliefs and church organizations that believe in spiritual management of health and pregnancies.

Efficacy. The capacity of the MP to produce the desired therapeutic effect.

Isihlambezo. A herbal decoction used among the Zulu women in South Africa as a preventative health tonic during pregnancy. Most common Isihlambezo plants: Agapanthus africanus (L.) Hoffmanns.; Asclepias fruticosa L.; Callilepis laureola DC.; Clivia miniata (Lindl.) Bosse; Combretum erythrophyllum (Burch.) Sond.; Crinum macowanii Baker; Gunnera perpensa L.; Pentanisia prunelloides (Klotzsch) Walp.; Rhoicissus tridentata (L.f.) Wild \& R.B.Drumm.; Scadoxus puniceus (L.) Friis \&
Nordal; Typha capensis (Rohrb) N.E Br.; Gymnanthemum corymbosum (Thunb.) H.Rob.; Ruta graveolens L.; Dioscorea dregeana (Kunth) T.Durand \& Schinz; Eucomis comosa (Houtt.) Wehrh.; Gnidia kraussiana Meisn.; Cyrtanthus obliquus (L.f.) Aiton.; Gladiolus sericeovillosus Hook.f.; Grewia occidentalis L.; Plantago major L.. Isihlambezo is generally prepared by boiling the plant materials in water for approximately 30 40 minutes to produce a decoction.

Mitch. A febrile illness believed to be caused by excessive sunlight and manifested by swelling and/or formation of sores on parts of the human body. It is almost similar to cold sore.

Safety. The freedom from potential adverse effects related to the administration of MPs.

Therapeutic meal. A preparation of mixed medicinal plants (e.g. Elaeis guineensis Jacq.) and animal (e.g. Chrysichthys nigrodigitatus) products.

\section{Appendix A. Supporting information}

Supplementary data associated with this article can be found in the online version at http://dx.doi.org/10.1016/j.jep.2018.05.032.

\section{References}

Abasiubong, F., Bassey, E.A., Udobang, J.A., Akinbami, O.S., Udoh, S.B., Idung, A.U., 2012. Self-Medication: potential risks and hazards among pregnant women in Uyo, Nigeria. Pan Afr. Med. J. 13, 15.

Abdullahi, A.A., 2011. Trends and challenges of traditional medicine in Africa. African. J. Tradit. Complement. Altern. Med. 8, 115-123.

Abeje, G., Admasie, C., Wasie, B., 2015. Factors associated with self medication practice among pregnant mothers attending antenatal care at governmental health centers in Bahir Dar city administration, Northwest Ethiopia, a cross sectional study. Pan Afr. Med. J. 20, 276.

Aboyeji, A.P., Ijaiya, M.A., Fawole, A.A., Adesina, K.T., Ologe, M.O., Adewara, A.A. Olarinoye, A.O., 2014. Pharmacological exposure of pregnant mothers in Ilorin, Nigeria. Tropical. J. Obstet. Gynaecol. 31, 17-22.

Addo, V.N., 2007. Herbal medicines: socio-demographic characteristics and pattern of use by patients in a tertiary obstetrics and gynaecology unit. J. Sci. Technol. 27, 149-155.

Adnan, M., Gul, S., Batool, S., Fatima, B., Rehman, A., Yaqoob, S., Shabir, H., Yousaf, T., Mussarat, S., Ali, N., Khan, S.N., Rahman, H., Aziz, M.A., 2017. A review on the ethnobotany, phytochemistry, pharmacology and nutritional composition of Cucurbita pepo L. J. Phytopharm. 6, 133-139.

Adusi-Poku, Y., Vanotoo, L., Detoh, E.K., Oduro, J., Nsiah, R.B., Natogmah, A.Z, 2015. Type of herbal medicines utilized by pregnant women attending Ante-Natal Clinic in Offinso North District: are orthodox prescribers aware? Ghana Med. J. 49, 227-232.

Agyei-Boateng, R., 2015. Self-Medication Practices among Pregnant Women in EjisuJuaben Municipality (M.Sc. Thesis). Kwame Nkrumah University of Science and Technology, Kumasi, Ghana (Unpublished).

Alaku, I.A., Abdullahi, A.G., 2015. Epidemiology of malaria parasites infection among pregnant women in some part of Nasarawa State, Nigeria. Dev. Ctry. Stud. 5, 30-34.

Alebie, G., Urga, B., Worku, A., 2017. Systematic review on traditional medicinal plants used for the treatment of malaria in Ethiopia: trends and perspectives. Malar J. 16, 307

Alor, S.K., 2015. The Use of Traditional Medicine among Pregnant Women in AgotimeZiope District (M.Sc. Thesis). University of Ghana, Accra, Ghana (Unpublished).

Anderson, L.M., Scrimshaw, S.C., Fullilove, M.T., Fielding, J.E., Normand, J., 2003. Culturally competent healthcare systems: a systematic review. Am. J. Prev. Med. 24, 68-79.

Auer, W., Eiber, A., Hertkorn, E., Hoehfeld, E., Koehrle, U., Lorenz, A., Mader, F., Merx, W., Otto, G., Schmid-Otto, B., 1990. Hypertension and hyperlipidaemia: garlic helps in mild cases. Br. J. Clin. Pract. 69, 3-6.

Bayisa, B., Tatiparthi, R., Mulisa, E., 2014. Use of herbal medicine among pregnant women on Antenatal care at Nekemte Hospital, Western Ethiopia. Jundishapur J. Nat. Pharm. Prod. 9, 1-5.

Bello, F.A., Morhason-Bello, I.O., Olayemi, O., Adekunle, A.O., 2011. Patterns and predictors of self-medication amongst antenatal clients in Ibadan, Nigeria. Niger. Med. J. 52, 153-157.

Bello, U.L., Isah, J., 2015. Use of Herbal Medicines and Aphrodisiac Substances among Women in Kano State, Nigeria. IOSR J. Nurs. Health Sci. 4, 41-50.

Bishaw, M., 1991. Promoting traditional medicine in Ethiopia: a brief historical review of government policy. Soc. Sci. Med. 33, 193-200.

Black, R.A., Hill, D.A., 2003. Over-the-counter medications in pregnancy. Am. Fam. Phys. $67,2517-2524$

Boel, M.E., Lee, S.J., Rijken, M.J., Paw, M.K., Pimanpanarak, M., Tan, S.O., Pratap Singhasivanon, P., Nosten, F., McGready, R., 2009. Castor oil for induction of labour: not harmful, not helpful. Aust. N.Z. J. Obstet. Gynaecol. 49, 499-503.

de Boer, H.J., 2009. African plants as antipathogen agents: efficacy and clinical evidence. In: Watson, R., Preedy, V. (Eds.), Botanical Medicine in Clinical Practice. CABI, UK, pp. 3-12.

de Boer, H.J., Cotingting, C., 2014. Medicinal plants for women's healthcare in southeast 
Asia: a meta-analysis of their traditional use, chemical constituents, and pharmacology. J. Ethnopharmacol. 151, 747-767.

de Boer, H.J., Lamxay, V., 2009. Plants used during pregnancy, childbirth and postpartum healthcare in Lao PDR: a comparative study of the Brou, Saek and Kry ethnic groups. J. Ethnobiol. Ethnomed. 5, 25.

de Boer, H.J., Lamxay, V., Björk, L., 2012. Comparing medicinal plant knowledge using similarity indices: a case of the Brou, Saek and Kry in Lao PDR. J. Ethnopharmacol. $141,481-500$.

Boltman-Binkowski, H., 2016. A systematic review: are herbal and homeopathic remedies used during pregnancy safe? Curationis 39, 1514.

Camou-Guerrero, A., Reyes-García, V., Martínez-Ramos, M., Casas, A., 2008. Knowledge and use value of plant species in a Rarámuri Community: a gender perspective for conservation. Hum. Ecol. 36, 259-272.

Charlson, M., McFerren, M., 2007. Garlic: what we know and what we don't know. Arch. Intern. Med. 167, 325-326.

Chukwurah, J.N., Idowu, E.T., Adeneye, A.K., Aina, O.O., Agomo, P.U., Otubanjo, A.O., 2016. Knowledge, attitude and practice on malaria prevention and sulfadoxine pyrimethamine utilisation among pregnant women in Badagry, Lagos State, Nigeria. Malar. World J. 7, 1-6.

Ciganda, C., Laborde, A., 2003. Herbal infusions used for induced abortion. J. Toxicol.: Clin. Toxicol. 41, 235-239.

Cordell, G.A., 2015. Ecopharmacognosy and the globalization of traditional medicines. Indian J. Tradit. Knowl. 14, 595-604.

Dante, G., Pedrielli, G., Annessi, E., Facchinetti, F., 2013. Herb remedies during pregnancy: a systematic review of controlled clinical trials. J. Matern.-Fetal Neonatal. Med. 26, 306-312.

Dante, G., Bellei, G., Neri, I., Facchinetti, F., 2014. Herbal therapies in pregnancy: what works? Curr. Opin. Obstet. Gynecol. 26, 83-91.

Duru, C.B., Uwakwe, K.A., Chinomnso, N.C., Mbachi, I.I., Diwe, K.C., Agunwa, C.C., Iwu, A.C., Merenu, I.A., 2016a. Socio-demographic determinants of herbal medicine use in pregnancy among Nigerian women attending clinics in a tertiary hospital in Imo State, South-East Nigeria. Am. J. Med. Stud. 4, 1-10.

Duru, C.B., Nnebue, C.C., Uwakwe, K.A., Diwe, K.C., Agunwa, C.C., Achigbu, K.I., Iwu, C.A., Merenu, I.A., 2016b. Prevalence and pattern of herbal medicine use in pregnancy among women attending clinics in a tertiary hospital in Imo State, South East Nigeria. Int. J. Curr. Res. Biosci. Plant Biol. 3, 5-14.

Emmanuel, A., Achema, G., Afoi, B.B., Maroof, R., 2014. Self medication practice among pregnant women attending antenatal clinic in selected hospitals in Jos, Nigeria. Int. J. Nurs. Health Sci. 1, 55-59.

Ernst, E., 2002. Herbal medicinal products during pregnancy: are they safe? BJOG: Int. J. Obstet. Gynaecol. 109, 227-235.

Ernst, E., Pittler, M.H., 2000. Efficacy of ginger for nausea and vomiting: a systematic review of randomized clinical trials. Br. J. Anaesth. 84, 367-371.

Fakeye, T.O., Adisa, R., Musa, I.E., 2009. Attitude and use of herbal medicines among pregnant women in Nigeria. BMC Complement. Altern. Med. 9, 53.

Fischer-Rasmussen, W., Kjaer, S.K., Dahl, C., Asping, U., 1991. Ginger treatment of hyperemesis gravidarum. Eur. J. Obstet. Gynecol. Reprod. Biol. 38, 19-24.

Forster, D.A., Denning, A., Wills, G., Bolger, M., McCarthy, E., 2006. Herbal medicine use during pregnancy in a group of Australian women. BMC Pregnancy Childbirth 6, 21.

Frawley, J., Adams, J., Sibbritt, D., Steel, A., Broom, A., Gallois, C., 2013. Prevalence and determinants of complementary and alternative medicine use during pregnancy: results from a nationally representative sample of Australian pregnant women. Aust. N.Z. J. Obstet. Gynaecol. 53, 347-352.

Fwacs, O.A., Fwacs, A.T.A., 2013. Use of herbal medicines in Pregnancy in Aminu Kano teaching hospital-Myth or Reality? Tropical. J. Obstet. Gynaecol. 30, 80-88.

Gall, A., Shenkute, Z., 2009. Ethiopian Traditional and Herbal Medications and Their Interactions with Conventional Drugs. 〈https://ethnomed.org/clinical/pharmacy/ ethiopian-herb-drug-interactions (Accessed 15 October 2016).

Garry, D., Figueroa, R., Guillaume, J., Cucco, V., 2000. Use of castor oil in pregnancies at term. Altern. Ther. Health Med. 6, 77-79.

Gebremedhin, G.L., Gomathi, P., 2014. Assessment of drug use and effect in pregnan women attending antenatal care in hospitals of Mekelle, Tigray, Ethiopia. J. Drug Deliv. Ther. 4, 75-82.

Gedif, T., Hahn, H.-J., 2002. Herbalists in Addis Ababa and Butajira, central Ethiopia: mode of service delivery and traditional pharmaceutical practice. Ethiop. J. Health Dev. 16, 191-197.

Gharoro, E.P., Igbafe, A., 2000. Pattern of drug use amongst antenatal patients in Benin City, Nigeria. Med. Sci. Monit. 6, 84-87.

Gibson, P.S., Powrie, R., Star, J., 2001. Herbal and alternative medicine use during pregnancy: a cross-sectional survey. Obstet. Gynecol. 97, S44-S45.

Glover, D.D., Amonkar, M., Rybeck, B.F., Tracy, T.S., 2003. Prescription, over-thecounter, and herbal medicine use in a rural, obstetric population. Am. J. Obstet. Gynecol. 188, 1039-1045.

Godlove, M.J., 2011. Prevalence of Herbal Medicine Use and Associated Factors among Boer, Pregnant Women Attending Antenatal Clinic at Mbeya Refferal Hospital in 2010 (M.Sc. Thesis). Muhimbili University of Health and Allied Sciences, Dar es salaam, Tanzania (Unpublished).

Govaerts, R., 2001. How many species of seed plants are there? Taxon 50, 1085-1090.

Guh, J.-H., Ko, F.-N., Jong, T.-T., Teng, C.M., 1995. Antiplatelet effect of Gingerol isolated from Zingiber officinale. J. Pharm. Pharmacol. 47, 329-332.

Haeri, S., Dildy, G.A., 2012. Maternal mortality from hemorrhage. Semin. Perinatol. 36, $48-55$.

Hall, H.G., Griffiths, D.L., McKenna, L.G., 2011. The use of complementary and alternative medicine by pregnant women: a literature review. Midwifery 27, 817-824.

Hanafy, S.A., Sallam, S.A., Kharboush, I.F., Wahdan, I.H., 2016. Drug utilization pattern during pregnancy in Alexandria, Egypt. Eur. J. Pharm. Med. Res. 3, 19-29.
Heitmann, K., Nordeng, H., Holst, L., 2013. Safety of ginger use in pregnancy: results from a large population-based cohort study. Eur. J. Clin. Pharmacol. 69, 269-277.

Hepner, D.L., Harnett, M., Segal, S., Camann, W., Bader, A.M., Tsen, L.C., 2002. Herbal medicine use in parturients. Anesth. Analg. 94, 690-693.

Hillary, N.C., 2013. Utilization of Herbal Medicine during Pregnancy, Labour and Postpartum Period among Women at Embu Provincial General Hospital (M.Sc. Thesis). Nairobi University, Nairobi, Kenya (Unpublished).

Hollyer, T., Boon, H., Georgousis, A., Smith, M., Einarson, A., 2002. The use of CAM by women suffering from nausea and vomiting during pregnancy. BMC Complement. Altern. Med. 2, 5.

Holst, L., Wright, D., Nordeng, H., Haavik, S., 2009. Use of herbal preparations during pregnancy: focus group discussion among expectant mothers attending a hospital antenatal clinic in Norwich, UK. Complement. Ther. Clin. Pract. 15, 225-229.

Holst, L., Wright, D., Haavik, S., Nordeng, H., 2011. Safety and efficacy of herbal remedies in obstetrics-review and clinical implications. Midwifery 27, 80-86.

Izzo, A.A., 2012. Interactions between herbs and conventional drugs: overview of the clinical data. Med. Princ. Pract. 21, 404-428.

John, L.J., Shantakumari, N., 2015. Herbal medicines use during pregnancy: a review from the middle east. Oman Med. J. 30, 229-236.

Joseph, B.N., Ezie, I.J., Aya, B.M., Dapar, M.L.P., 2017. Self-medication among pregnant women attending ante-natal clinics in Jos-North, Nigeria. Int. J. Trop. Dis. Health $21,1-7$.

Kaingu, C.K., Oduma, J.A., Kanui, T.I., 2011. Practices of traditional birth attendants in Machakos District, Kenya. J. Ethnopharmacol. 137, 495-502.

Kamatenesi-Mugisha, M., Oryem-Origa, H., 2007. Medicinal plants used to induce labour during childbirth in western Uganda. J. Ethnopharmacol. 109, 1-9.

Kebede, B., Gedif, T., Getachew, A., 2009. Assessment of drug use among pregnant women in Addis Ababa, Ethiopia. Pharmacoepidemiol. Drug Saf. 18, 462-468.

Kennedy, D.A., Lupattelli, A., Koren, G., Nordeng, H., 2013. Herbal medicine use in pregnancy: results of a multinational study. BMC Complement. Altern. Med. 13, 355.

Khan, K.S., Wojdyla, D., Say, L., Gülmezoglu, A.M., Van Look, P.F., 2006. WHO analysis of causes of maternal death: a systematic review. Lancet 367, 1066-1074.

Kinney, M.V., Kerber, K.J., Black, R.E., Cohen, B., Nkrumah, F., Coovadia, H., Nampala, P.M., Lawn, J.E., 2010. Sub-Saharan Africa's mothers, newborns, and children: where and why do they die? PLoS Med. 7, e1000294.

Laelago, T., Yohannes, T., Lemango, F., 2016. Prevalence of herbal medicine use and associated factors among pregnant women attending antenatal care at public health facilities in Hossana Town, Southern Ethiopia: facility based cross sectional study. Arch. Public Health (74:7).

Legesse, Y., Tegegn, A., Belachew, T., Tushune, K., 2007. Knowledge, attitude and practice about malaria transmission and its preventive measures among households in urban areas of Assosa Zone, Western Ethiopia. Ethiop. J. Health Dev. 21, 157-165.

Maats, F.H., Crowther, C.A., 2002. Patterns of vitamin, mineral and herbal supplement use prior to and during pregnancy. Aust. N.Z. J. Obstet. Gynaecol. 42, 494-496.

Mabina, M.H., Pitsoe, S.B., Moodley, J., 1997b. The effect of traditional herbal medicines on pregnancy outcome. S. Afr. Med. J. 87, 1008-1010.

Mabina, M.H., Moodley, J., Pitsoe, S.B., 1997a. The use of traditional herbal medication during pregnancy. Trop. Dr. 27, 84-86.

Makita-Ikouaya, E., Mombo, J.B., Rudant, J.P., Milleliri, J.M., 2010. Availability and access to the public healthcare facilities in sub-Saharan Africa: situation in Libreville, Gabon. Med. Trop. 70, 123-129.

Malan, D.F., Neuba, D.F., 2011. Traditional practices and medicinal plants use during pregnancy by Anyi-Ndenye women (Eastern Côte d'Tvoire). Afr. J. Reprod. Health 15, $85-93$.

Mathee, A., Naicker, N., Kootbodien, T., Mahuma, T., Nkomo, P., Naik, I., de Wet, T., 2014. A cross-sectional analytical study of geophagia practices and blood metal concentrations in pregnant women in Johannesburg, South Africa. S. Afr. Med. J. 104, 568-573.

Mbarambara, P.M., Songa, P.B., Wansubi, L.M., Mututa, P.M., Minga, B.B.K., 2016. Selfmedication practice among pregnant women attending antenatal care at health centers in Bukavu, Eastern DR Congo. Int. J. Innov. Appl. Stud. 16, 38-45.

Mbura, J.S., Mgaya, H.N., Heggenhougen, H.K., 1985. The use of oral herbal medicine by women attending antenatal clinics in urban and rural Tanga District in Tanzania. East Afr. Med. J. 62, 540-550.

McKay, D.L., Blumberg, J.B., 2006. A review of the bioactivity and potential health benefits of peppermint tea (Mentha piperita L.). Phytother. Res. 20, 619-633.

Mkize, G.T., 2015. An Assessment of Use of Traditional Medicine in Pregnancy \& Associated Factors Among Black South African Women Delivering in Bertha Gxowa Hospital (M.Sc. Thesis). University of the Witwatersrand, Johannesburg, South Africa (Unpublished)

Mokgobi, M.G., 2014. Understanding traditional African healing. Afr. J. Phys. Health Educ. Recreat. Dance 20, 24-34.

Mothupi, M.C., 2014. Use of herbal medicine during pregnancy among women with access to public healthcare in Nairobi, Kenya: a cross-sectional survey. BMC Complement. Altern. Med. 14, 432.

Moussally, K., Oraichi, D., Be'rard, A., 2009. Herbal products use during pregnancy: prevalence and predictors. Pharmacoepidemiol. Drug Saf. 18, 454-461.

Mugomeri, E., Chatanga, P., Seliane, K., Maibvise, C., 2015. Identifying promoters and reasons for medicinal herb usage during pregnancy in Maseru, Lesotho. Afr. J. Nurs. Midwifery 17, 4-16.

Mureyi, D.D., Monera, T.G., Maponga, C.C., 2012. Prevalence and patterns of prenatal use of traditional medicine among women at selected harare clinics: a cross-sectional study. BMC Complement. Altern. Med. 12, 164.

Mussa, S., Gedif, T., 2011b. Knowledge About Malaria, and Coverage and Utilization Pattern of Mosquito Nets in Pawe Woreda, Northwest Ethiopia: A Community Based Survey. Ethiop. Pharm. J. 29, 68-78. 
Mussa, S., Gedif, T., 2011a. Antimalarial drug use pattern in pawe special Woreda, North West Ethiopia: a community based survey. Ethiop. Pharm. J. 29 (2), 121-136.

Nergard, C.S., Ho, T.P.T., Diallo, D., Ballo, N., Paulsen, B.S., Nordeng, H., 2015. Attitudes and use of medicinal plants during pregnancy among women at health care centers in three regions of Mali, West-Africa. J. Ethnobiol. Ethnomed. 11, 73.

Njamen, D., Mvondo, M.A., Djiogue, S., Wanda, G.J.M.K., Nde, C.B.M., Vollmer, G., 2013. Phytotherapy and women^s reproductive health: the Cameroonian perspective. Planta Med. 79, 600-611.

Nordeng, H., Havnen, G.C., 2004. Use of herbal drugs in pregnancy: a survey among 400 Norwegian women. Pharmacoepidemiol. Drug Saf. 13, 371-380.

Nordeng, H., Bayne, K., Havnen, G.C., Paulsen, B.S., 2011. Use of herbal drugs during pregnancy among 600 Norwegian women in relation to concurrent use of conventional drugs and pregnancy outcome. Complement. Ther. Clin. Pract. 17, 147-151.

Nordeng, H., Al-Zayadi, W., Diallo, D., Ballo, N., Paulsen, B.S., 2013. Traditional medicine practitioners' knowledge and views on treatment of pregnant women in three regions of Mali. J. Ethnobiol. Ethnomed, 9, 67.

Nyeko, R., Tumwesigye, N.M., Halage, A.A., 2016. Prevalence and factors associated with use of herbal medicines during pregnancy among women attending postnatal clinics in Gulu district, Northern Uganda. BMC Pregnancy Childbirth 16, 296.

Oladeinde, B.H., Omoregie, R., Odia, I., Oladeinde, O.B., 2012. Prevalence of malaria and anemia among pregnant women attending a traditional birth home in Benin city, Nigeria. Oman Med. J. 27, 232-236.

Olowokere, A.E., Olajide, O., 2013. Women's perception of safety and utilization of herbal remedies during pregnancy in a local government area in Nigeria. Clin. Nurs. Stud. 1, $9-22$.

Omane-Adjekum, W., 2010. Medicines Usage in Pregnancy (M.Sc. thesis). Kwame Nkrumah University of Science and Technology, Kumasi, Ghana (Unpublished).

Onyiapat, J.L.E., 2014. Complementary and Alternative Medicine Use among Pregnan Women in Udi Local Government Area of Enugu State, Nigeria (M.Sc. thesis). University of Nigeria, Enugu Campus, Nigeria (Unpublished).

Opaneye, A.A., 1998. Traditional medicine in Nigeria and modern obstetric practice: need for cooperation. Cent. Afr. J. Med. 44, 258-261.

Orief, Y.I., Farghaly, N.F., Ibrahim, M.I.A., 2014. Use of herbal medicines among pregnant women attending family health centers in Alexandria. Middle East Fertil. Soc. J. 19, $42-50$

Pfeiffer, J.M., Butz, R.J., 2005. Assessing cultural and ecological variation in ethnobiological research: the importance of gender. J. Ethnobiol. 25, 240-278.

Pinn, G., Pallett, L., 2002. Herbal medicine in pregnancy. Complement. Therap. Nurs. Midwifery 8, 77-80.

Posadzki, P., Watson, L.K., Ernst, E., 2013. Adverse effects of herbal medicines: an overview of systematic reviews. Clin. Med. 13, 7-12.

Rahman, A.A., Ahmad, Z., Naing, L., Sulaiman, S.A., Hamid, A.M., Daud, W.N.W., 2007. The use of herbal medicines during pregnancy and perinatal mortality in Tumpat District, Kelantan, Malaysia. Southeast Asian J. Trop. Med. Public Health 38, 1150-1157.

Rasch, V., Srensen, P.H., Wang, A.R., Tibazarwa, F., Jger, A.K., 2014. Unsafe abortion in rural Tanzania - the use of traditional medicine from a patient and a provider perspective. BMC Pregnancy Childbirth 14, 419.

Rodrigues, E., 2007. Plants of restricted use indicated by three cultures in Brazil (Cabocloriver dweller, Indian and Quilombola). J. Ethnopharmacol. 111, 295-302.

de Sa, R.Z., Rey, A., Argañaraz, E., Bindstein, E., 2000. Perinatal toxicology of Ruta chalepensis (Rutaceae) in mice. J. Ethnopharmacol. 69, 93-98.

Sam-Wobo, S.O., Akinboroye, T., Anosike, J.C., Adewale, B., 2008. Knowledge and practices on malaria treatment measures among pregnant women in Abeokuta, Nigeria. Tanzan. J. Health Res. 10, 226-231.

Say, L., Chou, D., Gemmill, A., Tunçalp, Ö., Moller, A.B., Daniels, J., Gülmezoglu, A.M., Temmerman, M., Alkema, L., 2014. Global causes of maternal death: a who systematic analysis. Lancet Glob. Health 2, e323-e333.

Skouteris, H., Wertheim, E.H., Rallis, S., Paxton, S.J., Kelly, L., Milgrom, J., 2008. Use of complementary and alternative medicines by a sample of Australian women during pregnancy. Aust. N.Z. J. Obstet. Gynaecol. 48, 384-390.

Spolarich, A.E., Andrews, L., 2007. An examination of the bleeding complications associated with herbal supplements, antiplatelet and anticoagulant medications. J. Dent. Hyg. 81, 67.

Steenkamp, V., 2003. Traditional herbal remedies used by South African women for gynaecological complaints. J. Ethnopharmacol. 86, 97-108.

Suswardany, D.L., Sibbritt, D.W., Supardi, S., Chang, S., Adams, J., 2015. A critical review of traditional medicine and traditional healer use for malaria and among people in malaria-endemic areas: contemporary research in low to middle-income Asia-Pacific countries. Malar. J. 14, 98
Tamuno, I., Omole-Ohonsi, A., Fadare, J., 2011. Use of herbal medicine among pregnant women attending a tertiary hospital in Northern Nigeria. Internet J. Gynecol. Obstet. $15,2$.

Tardío, J., Pardo-de-Santayana, M., 2008. Cultural importance indices: a comparative analysis based on the useful wild plants of Southern Cantabria (Northern Spain). Econ. Bot. 62, 24-39.

Tate, S., 1997. Peppermint oil: a treatment for postoperative nausea. J. Adv. Nurs. 26, $543-549$.

Tongo, O.O., Orimadegun, A.E., Akinyinka, O.O., 2011. Utilisation of malaria preventive measures during pregnancy and birth outcomes in Ibadan, Nigeria. BMC Pregnancy Childbirth 11, 60 .

Tort, J., Rozenberg, P., Traoré, M., Fournier, P., Dumont, A., 2015. Factors associated with postpartum hemorrhage maternal death in referral hospitals in Senegal and Mali: a cross-sectional epidemiological survey. BMC Pregnancy Childbirth 15, 235

Towns, A.M., van Andel, T., 2014. Comparing local perspectives on women's health with statistics on maternal mortality: an ethnobotanical study in Bénin and Gabon. BMC Complement. Altern. Med. 14, 113.

Towns, A.M., van Andel, T., 2016. Wild plants, pregnancy, and the food-medicine continuum in the southern regions of Ghana and Benin. J. Ethnopharmacol. 179, 375-382.

Tsui, B., Dennehy, C.E., Tsourounis, C., 2001. A survey of dietary supplement use during pregnancy at an academic medical center. Am. J. Obstet. Gynecol. 185, 433-437.

USAID, 2012. Cultural Barriers to Seeking Maternal Health Care in Ethiopia: A Review of the Literature. 〈http://pdf.usaid.gov/pdf_docs/PA00JSN8.pdf〉 (Accessed 15 November 2017)

van Andel, T., de Boer, H.J., Barnes, J., Vandebroek, I., 2014. Medicinal plants used for menstrual disorders in Latin America, the Caribbean, sub-Saharan Africa, South and Southeast Asia and their uterine properties: a review. J. Ethnopharmacol. 155, 992-1000.

Varga, C.A., Veale, D.J.H., 1997. Isihlambezo: utilization patterns and potential health effects of pregnancy-related traditional herbal medicine. Social. Sci. Med. 44 911-924.

Veale, D.J.H., Furman, K.I., Oliver, D.W., 1992. South African traditional herbal medicines used during pregnancy and childbirth. J. Ethnopharmacol. 36, 185-191.

Verma, P., Thakur, A.S., Deshmukh, K., Jha, A.K., Verma, S., 2010. Routes of drug administration. Int. J. Pharm. Stud. Res. 1, 54-59.

Viljoen, E., Visser, J., Koen, N., Musekiwa, A., 2014. A systematic review and metaanalysis of the effect and safety of ginger in the treatment of pregnancy-associated nausea and vomiting. Nutr. J. 13, 20.

Wen, S.W., Yang, T., Krewski, D., Yang, Q., Nimrod, C., Garner, P., Fraser, W., Olatunbosun, O., Walker, M.C., 2008. Patterns of pregnancy exposure to prescription FDA C, D and X drugs in a Canadian population. J. Perinatol. 28, 324.

Westfall, R.E., 2003. Herbal healing in pregnancy: women's experiences. J. Herbal. Pharmacother. 3, 17-39.

WHO, 1991. Maternal Mortality: A Global Factbook. 〈http://www.popline.org/node/ 331283> (Accessed 01 November 2017).

WHO, 2000. General Guidelines for Methodologies on Research and Evaluation of Traditional Medicine. 1. WHO, Geneva, pp. 1-74. 〈https://doi.org/WHO/EDM/ TRM/2000.1> (accessed 03 October 2016).

WHO, 2002. WHO Traditional Medicine Strategy 2002-2005. World Health Organization, Geneva, Switzerland.

WHO, 2014. WHO policy brief for the Implementation of Intermittent Preventive Treatment of Malaria in Pregnancy Using Sulfadoxine-Pyrimethamine (IPTp-SP). 〈https://doi.org/WHO/HTM/GMP/2014.4〉 (Accessed 10 October 2017).

WHO, 2015. Trends in maternal mortality: 1990 to 2015: estimates by WHO, UNICEF, UNFPA, World Bank Group and the United Nations Population Division. World Health Organization, Geneva. 〈http://apps.who.int/iris/bitstream/handle/10665/ 194254/9789241565141_eng.pdf;jsessionid $=$ 64E023BC4DE93EDB3A075E4E67F47DB5?Sequence $=$ 1> (Accessed 13 October 2017).

Wills, G., Forster, D., 2008. Nausea and vomiting in pregnancy: what advice do midwives give? Midwifery 24, 390-398.

Yue, Q.Y., Jansson, K., 2001. Herbal drug curbicin and anticoagulation effect with and without warfarin: possibly related to the vitamin E component. J. Am. Geriatr. Soc. 49 (838-838).

Yusuff, K.B., Omarusehe, L.D., 2011. Determinants of self medication practices among pregnant women in Ibadan, Nigeria. Int. J. Clin. Pharm. 33, 868-875.

Zhang, Y., Xu, H., Chen, H., Wang, F., Huai, H., 2014. Diversity of wetland plants used traditionally in China: a literature review. J. Ethnobiol. Ethnomed. 10, 72. 

II 

Medicinal plants used among pregnant women in a tertiary teaching hospital in Jimma,

4 Seid Mussa Ahmed ${ }^{1,2 *}$, Johanne Sundby ${ }^{1}$, Yesuf Ahmed Aragaw ${ }^{3}$, Hedvig Nordeng 4

$5 \quad{ }^{1}$ Department of Community Medicine and Global Health, Institute of Health and Society,

6 Faculty of Medicine, University of Oslo, Norway;

$7 \quad{ }^{2}$ Division of Social and Administrative Pharmacy, School of Pharmacy, Faculty of Health

8 Sciences, Institute of Health, Jimma University, Ethiopia;

$9{ }^{3}$ Department of Obstetrics and Gynaecology, Faculty of Medical Sciences, Institute of Health, 10 Jimma University, Ethiopia;

$11{ }^{4}$ Pharmacoepidemiology and Drug Safety Research Group, Department of Pharmacy, Faculty of Mathematics and Natural Sciences, University of Oslo, Norway

* Corresponding author

E-mail: seidma@studmed.uio.no

Postal address: P.O box 1130, Blindern, 0318 OSLO, Norway

Fax number: $+47-22850590$

Telephone (Office): +47-22850688

16-digit ORCID: 0000-0003-3426-5296

E-mail addresses:

SMA: seidma@studmed.uio.no

HN: h.m.e.nordeng@farmasi.uio.no

JS: johanne.sundby@medisin.uio.no

YAA: yesufahmed47@yahoo.com 
Abstract

Objective The aim of this study was to investigate and describe the use of medicinal plants during pregnancy among women admitted in the Maternity and Gynaecology wards at Jimma University Medical Centre (JUMC) in the southwest Ethiopia.

\section{Design Cross-sectional study}

Setting Maternity and Gynaecology wards at JUMC.

Participants 1,117 hospitalized pregnant women or postpartum women

Main outcome measures our primary outcomes of interest were the prevalence of use, types of medicinal plants used and their utilization among pregnant women.

Methods: Data were collected through structured face-to-face interviews of pregnant women or postpartum women and review of patient medical records between February and June 2017. Results: Overall, $28.6 \%$ of the women reported use of at least one medicinal plant during pregnancy. Twenty-seven different types of medicinal plants were used. The most commonly used medicinal plants were Linum usitatissimum L. (flaxseed- use with caution) $22.0 \%$, Ocimum lamiifolium L. (damakessie- safety unknown) 3.6\%, and Carica papaya L. (papayause with caution) $3.1 \%$. The most common reasons for use was preparation, induction or shortening of labour. Lack of access to health facility (mainly health posts), admission to maternity ward, khat chewing, and alcohol consumption were the strongest predictors of medicinal plants use during pregnancy $(\mathrm{OR}>2)$. Only five medicinal plants used by women had sufficient evidence to be classified as safe to use in pregnancy.

Conclusions: Almost a third of women at the tertiary hospital in Ethiopia reported use of medicinal plants during pregnancy, most frequently to prepare, induce, reduce the intensity or shorten duration of labour. Increased awareness about potential benefits or risks of medicinal plants use during pregnancy among health care professionals and patients, and increased access 
to childbirth providing health care facilities are important in order to promote safer pregnancies and better health outcomes for women and their unborn children.

\section{Strengths and limitations of this study}

- It was the first study in Ethiopia that used large sample size, assessed the use of medicinal plants among pregnant women in an in-patient setting and attempted to classify the medicinal plants

- Face-to-face interviews permitted the women to ask clarifying questions and ensured completeness and comprehension.

- The data collectors, pharmacists and nurses, were from the study area with previous data collection experience. Their knowledge about the healthcare system, culture, local languages, and medicinal plants was vital for the personal interviews with the women and clearly contributed to improving the response rate and the quality of collected data.

- Although it was conducted in a large tertiary teaching hospital in southwest Ethiopia, it may not be representative of the entire country, nor women who access healthcare in secondary or primary care.

- Data were collected based on self-report of pregnant women and thus depended on her recall and accuracy of reporting, as well as her knowledge about these medicinal plants, therefore, medicinal plant use early in pregnancy was probably underreported.

- Among the post-partum women, there may be a risk of recall bias as women with negative pregnancy outcomes may try to recall use to a greater extent than women with a healthy infant. 
Medicinal plants have been used for preventive and therapeutic purposes since time immemorial [1]. Medicinal plants refer to a variety of plants that have medicinal characteristics [2]. The World Health Organization estimates that $65-80 \%$ of the world's population in developing countries depend on medicinal plants for primary healthcare [3]. Women are recognized to be the main users of medicinal plants, and this widespread use also extends into pregnancy $[4,5]$.

Ethiopia is a landlocked country with a population of approximately 110 million [6]. It is a multi-ethnic, multicultural and multi-religious nation where Christians predominate in the northern highlands and central Ethiopia and Muslims predominate in the north-east, east, southeast and southwest [7]. More than $80 \%$ of the population lives in rural areas and $70 \%$ of the population are employed in agriculture [6]. The birth rate is 31 births per 1000 inhabitants and infant mortality rate is 35 deaths per 1000 live births [6]. Maternal mortality rate is high with 4 deaths per 1000 live births (world ranking 26th) [6]. Total fertility rate is 4 children born per woman, and mother's mean age at first birth is 20 years [6]. Physician density is only one per 12,500 inhabitants [6]. Around $80 \%$ of the population in Ethiopia use traditional medicine, of which over $95 \%$ are of plant origin [8]. The extensive use of medicinal plants in the country is often linked to an array of unique flora [8], cultural acceptability of healers and local pharmacopoeias, the belief that medicinal plants are natural and thus safer to use and are physically accessible and economically affordable $[4,5,9]$.

Maternal mortality (353 deaths per 1000,000 live births) and neonatal mortality (28 deaths per 1,000 live births) in Ethiopia are among the highest in the world and are associated with a range of factors [10]. In most African countries like Ethiopia, modern healthcare facilities and 
medicine are inaccessible or unaffordable $[4,11]$. For this reason, many women rely on medicinal plants for their primary healthcare needs as an accessible and lower cost alternative [4] and only seek professional health services when the situation worsens [11].

Studies conducted in Ethiopia reported prevalence of medicinal plants use in pregnancy ranging from $2 \%$ to $73 \%$ [4], with ginger being the most commonly used plant, and nausea and vomiting in pregnancy (NVP) and common cold the most common reasons for use $[9,11,12]$. Many sociodemographic characteristics including residence place, marital status, family size, education level, age, and employment status were found to be strong predictors of use $[4,12$ 15]. Prevalence figures ranging from $4 \%$ to $100 \%$ were reported in other African countries [4]. Studies in developed countries where medicinal plant traditions may play a less strong role also reported a widespread use of medicinal plants in pregnancy, with Australia 11\% - 56\% [16], the US and Canada 4\% - 96\% [16, 17], and Europe $0.9 \%$ - 69\% [16, 18].

Concerns have been raised about safety of medicinal plants during pregnancy [4, 18-21]. A recent multinational study reported that only $22 \%$ of the medicinal plants used by pregnant women were found safe to use in pregnancy [21]. Similarly, a study from Asia showed that only $39 \%$ of the most commonly used medicinal plants by expectant women were safe to use in pregnancy [19].

Although medicinal plants play a significant role in traditional medicine during pregnancy, childbirth and postpartum care $[4,20]$, research on their use in the management of pregnancy related illnesses is still largely limited $[4,12,22]$. The aims of this study were therefore to determine the prevalence of use and types of medicinal plants used among pregnant women admitted in the Maternity and Gynaecology wards at Jimma University Medical Centre 
(JUMC), Southwest Ethiopia. This included identifying women's information on the most commonly used medicinal plants, the reasons for use, and factors associated with such use. The secondary aims were to assess women safety concerns and, who recommended use of the medicinal plants during pregnancy.

\section{Subjects and methods}

\section{Study design and setting}

A hospital based cross-sectional study was conducted in the Maternity and Gynaecology wards at Jimma University Medical Centre (JUMC). JUMC is one of the oldest and largest public teaching University hospitals in the country located in Jimma city, 350 kilometres south-west of Addis Ababa (the capital city of Ethiopia) $[23,24]$. The referral hospital provides tertiary level medical care for about 20 million people coming from the whole south-west Ethiopia [23]. Obstetrics and Gynaecology department of the medical center has a patient load of approximately 7,600 inpatients and 11,600 outpatients each year with bed capacity of around 265 [24].

Obstetrics and Gynaecology department has two inpatient wards; Gynaecology ward and Maternity ward (which includes maternity, labour and delivery ward and maternity operation theatre) [23]. Obstetric patients with 28 weeks of pregnancy or higher as well as women in labour are admitted in the maternity ward. On the one hand, women with a gestational length of less than 28 weeks are cared for at the gynaecology ward. The gynaecology ward also manages and treats gynaecological disorders in non-pregnant women.

\section{Study population and sample size}


Hospitalized pregnant or postpartum women in the Maternity and Gynaecology wards at JUMC were invited to participate in the study during normal working hours. Participants were consecutively informed about the aim and procedures of the study and written informed consent was obtained from each study participant. Pregnant or postpartum patients aged $\geq 18$ years admitted in the Maternity/Labour and Gynaecology wards at the time of data collection and willing to participate were included in the study. On the other hand, women who were too ill to participate, hard of hearing, unable to speak or mentally disabled, under 18 years of age, admitted for less than four hours, and non-pregnant women admitted in the gynaecology ward were excluded from the study.

Single population proportion Kish formula [25] was used to determine the sample size based on the following assumptions; $50 \%$ expected prevalence medicinal plant use (since there is no previous study conducted on the prevalence of medicinal plant use among hospitalized pregnant patients prior to admission), 5\% level significance, $80 \%$ power, and an error margin of 3\%. After adding a 5\% non-response rate, a final sample size of 1,121 pregnant women was required.

\section{Data collection and procedures}

Hospitalized pregnant and post-partum women were consecutively interviewed from February to June 2017. A pre-tested interview guided structured questionnaire, based on interviews, and data extraction form were used for data collection. Nine trained pharmacists and nurses from the study area, with close supervision of one of the investigators, conducted all interviews and data extractions. The questionnaire contains questions about the women's background, pregnancy-related illnesses, and use of medicinal plants. 
175 After a thorough review of the literature [9, 12, 22, 26, 27], with special focus on prior studies in African countries, the authors developed the survey questionnaire. It was developed in English and then translated into Amharic and Afan Oromo languages (the predominant local languages) to suit the target population. The questionnaires were translated back into English by other persons to confirm the validity. Lecturers fluent in English and their own local language from Jimma University with previous experience of translating questionnaires performed the translation and back translation of the study questionnaire. The data collection tool was then piloted on a sample of 30 hospitalized pregnant or lactating women at Shenen Ghibe district hospital found in Jimma city, and based on the results from the pilot, list of 25 commonly used medicinal plants and open-ended questions were included. Plant scientific names were verified with The Plant List (www.theplantlist.org). Final version of the questionnaire contained 77 items, with multiple choice, and open-ended questions (Supplementary table 1).

Treatment related characteristics, pregnancy characteristics, pregnancy outcomes and other medical information were retrieved from patients' medical record using data extraction forms. Following the pre-test, the data extraction form required minor revisions to improve comprehension and order (Supplementary table 2).

\section{Measures}

Use of medicinal plant

Study participants were specifically asked about the use in pregnancy of 25 commonly used medicinal plants: Linum usitatissimum L., Ocimum lamiifolium L., Zingiber officinale Roscoe., Allium sativum L., Trigonella foenum-graecum L., Nigella sativa L., Ruta chalepensis L., Eucalyptus globulus Labill., Cinnamomum verum J.Presl, Taverniera abyssinica A. Rich, 
L., Datura stramonium L., and Securidaca longipedunculata Fresen. The above listed medicinal plants were selected based on previous ethnopharmacological studies in Ethiopia and elsewhere in Africa $[9,12,28,29]$ and were presented to the women by mentioning the local names of the plants. The women were also asked if they had used any other medicinal plant during pregnancy, labour or breastfeeding.

Details of use of medicinal plants was assessed by a series of questions including use of medicinal plant during pregnancy, type of medicinal plant used, reason for use, and utilization (part of plant used, method of preparation, mode of use, type of solvent, type of flavouring, dosage form, dosage, measures of formulation, route of administration, frequency of administration, duration of treatment, and episodes of use). Women were also asked about who recommended them the use of medicinal plants in pregnancy.

Information about women's safety concerns and experiences with use of medicinal plants in pregnancy was collected, and we included questions about beliefs about harmfulness, precautions to be taken and whether she had experienced any side effects or adverse effects after use of medicinal plants in pregnancy.

Reference text books [30-32] and literature reviews [4, 19, 21] were used to evaluate safety of the medicinal plants in pregnancy, and classify them into four safety categories, namely safe to use in pregnancy, use with caution, potentially harmful and information unavailable for use 
in pregnancy (Supplementary table 3). Information from animal studies were used if human studies were lacking. If a medicinal plant preparation was composed of two or more plants, each plant was individually evaluated and classified.

\section{Women's background characteristics}

Socio-demographic information including age, religion, residence place, occupation, family size, ethnic group, marital status, educational level, access to modern health facility and walking distance to the facility were collected.

\section{Maternal diseases, pregnancy-related illness and treatments}

Detailed information about the woman's obstetrics and gynaecology history, history of adverse pregnancy outcome, past medical history and medication experience, and social drug use were included. Pregnant women were specifically asked about 24 common pregnancy ailments and related symptoms: Common cold/flu, pain in back, neck, or shoulder, headache, heartburn/reflux problems, abdominal cramps/ache, preparation for labour, induction of labour, expel retained placenta, postpartum bathing, wellbeing and nourishing foetus, leg/foot swelling, gestational hypertension, gestational diabetes, gastritis/burning sensation, constipation, general wellbeing, nausea, vomiting, emergency illnesses, urinary tract infection, depression, joint pain, sleeping problems and mental wellbeing. Participants were also asked whether they had used any treatment against ailments or pregnancy related conditions, whether they had had any other diseases or illnesses, and, if yes, the name of any treatment received.

In addition to the face-to-face interview questionnaire, information about pregnancy characteristics, pregnancy outcomes and other obstetrics information including gestational age, parity, gravidity, mode of delivery and length of hospital stay were collected using a data 
extraction form. Moreover, maternal and perinatal outcomes of the current pregnancy were collected. Data were extracted through review of patients' medical cards.

\section{Statistical analysis}

254 The final data were checked for completeness, and responses were entered into and analysed using the Statistical Package for the Social Sciences (SPSS) software version 25.0 for Windows (IBM ${ }^{\circledR}$ SPSS ${ }^{\circledR}$ Statistics, Armonk). Respondents were categorized as users if they used at least one type of medicinal plant in their index pregnancy, whereas others were categorized as nonusers. Routine meals and vitamin supplements were excluded.

Descriptive statistics were used to calculate the prevalence $(\%)$ of medicinal plants use in pregnancy, reasons for use and information sources. Univariate and multivariate logistic regression analysis was used to identify significant factors associated with medicinal plant use. Logistic regression was expressed as crude and adjusted odds ratios (ORs) with 95\% confidence intervals (CIs). First, the univariate logistic regression model was fit for all explanatory variables. From this, the multivariate model was built using purposeful selection of candidate variables based on a bivariate $p \leq 0.05$. We then fit a reduced model by removing variables having no role $(\mathrm{p}>0.05)$. A $p$-value of $<0.05$ was considered statistically significant. Robustness of the multivariable model was checked using the Hosmer-Lemeshow test.

\section{Patient and public involvement}

271 Although there is a community representative in the Jimma University Institute of health Institutional Review Board (IRB), no patients or public were involved in the conception, design, conduct, and planning of this study. 


\section{Results}

276

277

From 1,137 pregnant or post-partum women invited to participate, responses from four were incomplete, and 16 declined to participate in the study resulting in 1,117 participants in the final dataset (response rate 98.6\%). The median age was 25 years (interquartile range 22-30 years) and slightly more than half (53.3\%), lived in an urban area. The majority were married (95.5\%), had access to health facility (mainly health post) $(99.1 \%)$, and lived in an area within walking distance to the nearest health facility not more than 30 minutes $(66.4 \%)$. A substantial number were Muslims (65.4\%), from Oromo ethnic group (69.7\%), and had a household size less than five (65.4\%). Many study participants were illiterate $(34.0 \%)$ or either attended primary school or only able to read \& write $(42.3 \%)$; and were housewife $(46.9 \%)$ or farmer (23.4\%) by occupation (Table 1$)$.

Table 1. Characteristics of women according to medicinal plant use during pregnancy at JUMC, Ethiopia.

In total, $28.6 \%$ women had used one or more medicinal plant during their current pregnancy, with an average of 1.5 medicinal plants per woman (range 1 to 8 ). The majority of women $206(64.6 \%)$ used one, $78(24.5 \%)$ took two, 25 (7.8\%) took three, and 7 (2.2\%) took four types of medicinal plants.

L. usitatissimum (flaxseed) (77.1\%), O. lamiifolium ('damakesie') (12.5\%) and $C$. papaya (papaya) (11.0\%) were the three most commonly used medicinal plants (Table 2; Supplementary table 4). The most common reasons for the use of medicinal plants were to induce labour or to reduce the intensity and shorten duration of labour (women call it

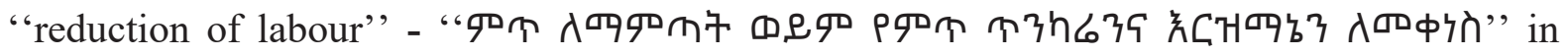
Amharic) (60.2\%) common cold/flu (20.4\%) and preparation of labour (women call 'it softens 


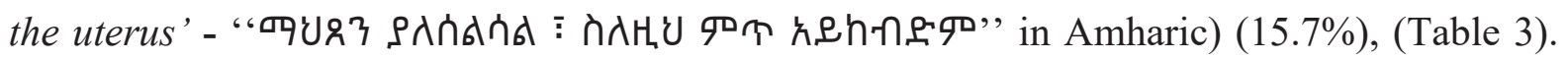

301 Flaxseed was the major plant employed to induce labour or to reduce the intensity and shorten

302

303

304

305

306

307

308

309

310

311

312

313

314

315

316

317

318

319

320

321

322

323

324 duration of labour (93.2\%) and to prepare for labour (44\%). Ginger (35.4\%) was the commonly used plant for common cold/flu management. Most of the medicinal plants were used during labour $(32.2 \%)$ followed by third trimester $(27.2 \%)$ or in the entire pregnancy $(19.8 \%)$.

Among the 125 women admitted to the gynaecology wards, 106 (84.8\%) were admitted due to elective terminations and/or miscarriages and $19(15.2 \%)$ were admitted due to various pregnancy-related illnesses. Among the women with elective terminations and / or miscarriages, 19 (17.9\%) women used one or more medicinal plants during pregnancy (range 1-3): 16 used safe, 9 used medicinal plants requiring cautious, 5 potentially harmful and 11 safety unknown medicinal plants. The 5 women who used potentially unsafe medicinal plants used Trigonella foenum-graecum (potential risk of uterine contraction and hypoglycemia), Ruta chalepensis (potential risk of uterine contraction and emmenagogue), Cinnamomum verum (potential risk of foetal malformation and uterine contraction), Artemisia abyssinica (potential risk of toxicity, uterine contraction and emmenagogue), Croton macrostachyus (potential risk of toxicity and uterine contraction), Echinops kebericho (potential risk of cytotoxicity) and Hagenia abyssinica (potential risk of toxicity and uterine contraction) (Supplementary table 4).

Approximately three quarters of the medicinal plants were purchased at market places (76.5\%). A significant proportion of respondents (68.3\%) also collected it through family members. The large majority of women were recommended to use medicinal plants by their family members $(75.2 \%)$.

Table 2. Pregnancy disorders treated with medicinal plants at JUMC, Ethiopia, $n=319$. 
Seeds were the major medicinal plant parts used (57.6\%), dry plant material was the most common plant condition (60.1\%), sugar was the most common excipient $(27.8 \%)$ and oral was the predominant route of administration $(89.7 \%)$.

The most common dosages were measurements by water glass units $(51.7 \%)$. The most common dosage was one water glass dose $(47.5 \%)$, once per day frequency $(54.8 \%)$, and "as many months as needed during pregnancy" duration of treatment (32.9\%). Approximately half of the respondents reported one episode of medicinal plant use (46.0\%), whereas nearly onethird reported use at several occasions during pregnancy 155 (32.0\%) (Supplementary table 5).

Table 3. Overview of the most frequently used medicinal plants during pregnancy according to number of users and the most common indications at JUMC, Ethiopia. secondary school education, having chronic illness, using conventional medicines and social drugs (khat chewers and alcohol consumers) were more likely to use medicinal plants in pregnancy (Table 1). Use of medicinal plants during pregnancy was not significantly associated with previous adverse pregnancy outcome, length of hospital stay, family size and gestational age.

\section{Safety classification of the medicinal plants}

347 From the 27 medicinal plants used by women, five were classified as safe to use, three as requiring caution to use, eight as potentially harmful to use in pregnancy and information on 
349 eleven medicinal plants was not available in the current literature. The names and safety 350 classification of the 27 individual medicinal plants are presented in Supplementary table 4.

351 Of those pregnant women who used medicinal plants, $14.4 \%$ used safe, $12.2 \%$ harmful, $3.4 \%$ both safe and harmful and $69.9 \%$ used one or more medicinal plants that requires cautious use or safety information unavailable. Many women who used safe or harmful medicinal plants have also used one or more plants that requires cautious use or safety information unavailable.

\section{Women's safety concerns and experiences}

Table 4 presents women's self-reported safety concerns and experiences with medicinal plants in pregnancy. Safety concerns with use in pregnancy was most commonly reported for bisana (C. macrostachyus) and astenagir (D. stramonium), each by five women. Four women reported drinking milk as antidote (“वपCh" officinale, T. abyssinica, H. abyssinica, and C. verum. Two women reported ingestion of $P$. anisum soup/suspension as countermeasure for poisoning from Z. officinale and C. verum. Eight women used L. usitatissimum for wellbeing and nourishing of the foetus. One woman reported the use of $O$. lamiifolium to improve foetal movements and breathing. O. lamiifolium, Z. officinale, and A. sativum were also reported to be useful for general foetal wellbeing. Fear of complications to the foetus $(44.5 \%)$ and religious prohibition $(25.9 \%)$ were the common reasons for avoiding use of medicinal plants during pregnancy.

Table 4. Pregnant women's self-reported safety concerns and experiences with medicinal plants at JUMC, Ethiopia

\section{Discussion}


373 Knowledge; both lay and professional, about medicinal plants use in pregnancy is essential to 374 provide optimal maternal/foetal care. To the best of our knowledge, this paper is the first to study medicinal plant use during pregnancy among women in an inpatient setting in Ethiopia. This study provides extensive insight into types of medicinal plants, prevalence of use and reasons for use, as well as women's safety concerns and precautions on the medicinal plants they use in pregnancy. These findings are important to health care personnel, researchers, policy makers, and pregnant women themselves. Nearly a third of women (28.6\%) reported use of at least one medicinal plant during pregnancy or at childbirth. Prior studies report global prevalence of use of medicinal plants in pregnancy ranging from $0.9 \%$ to $96.0 \%[4,16]$. Studies from Africa, however, report prevalence of medicinal plant use in pregnancy ranging from $2 \%$ (Ethiopia) to $100 \%$ (Kenya) [4]. Variation in prevalence may be explained by several factors including differences in study populations and settings, study inclusion and exclusion criteria as well as data collection methods and definitions of medicinal plants. In some studies, all forms of herbal meal preparations and nutritional supplements were counted [4] whereas in others, like our study, a more restrictive definition of medicinal plant use was used. In addition, differences in traditional practices, cultures and beliefs about health, may contribute to important difference in prevalence of use of medicinal plants.

The most frequently used medicinal plants during pregnancy were flaxseed (use with caution), damakessie (safety unknown) and papaya (use with caution, it is considered potentially unsafe in large amounts only) (Table 3, Supplementary table 4). Our finding is inconsistent with previous studies reported in Africa in which Z. officinale, A. sativum and C. pepo were the commonly used plants [4]. The pattern of medicinal plant use is also divergent from latest findings from Ethiopia $[13,14]$. This may be due to the fact that unlike previous studies, most participants in our study were women in their final stage of pregnancy and might most probably 
recall the medicinal plants they took in relation to childbirth to a better extent than plants used earlier in pregnancy. This difference in pattern of use from other corners of Ethiopia and regions elsewhere may be due to difference in climate, geographical location (which will affect the types of plants commonly grow in that area) and/or disease prevalence.

Flaxseed is by far the most commonly used medicinal plant, mainly used for induction, reduction, quickening or preparation for labour (Table 3). A recent study from Ethiopia had also found similar reason for its use [15]. In other African countries, however, seed oil from $R$. communis was the most frequently used medicinal plant product to stimulate labour [4]. The most probable reasons for the disparity in the type of medicinal plant used for labour induction may be differences in geographical distribution of plants and cultural beliefs.

In line with previous studies [33, 34], women reported side effects and safety concerns related to use of flaxseed in relation to labour (Table 4). A precautious consumption of flaxseed is recommended in pregnancy and lactation due to its side effects and adverse effects when consumed in excessive quantity [34]. In remote rural areas in Ethiopia where access to health facilities is limited, use of L. usitatissimum may be perceived as the best option to induce or shorten labour.

O. lamiifolium was the second most used medicinal plant during pregnancy in our study. It was mainly used for treatment of an illness called "Mitch" alone or with other medicinal plants (Table 2). "Mitch"' is a culturally common illness in Ethiopia and is a local name given to a febrile illness characterized by headache, fever, rash, inflammation, joint pain, back pain, chills, sweat, loss of appetite, Herpes labialis, muscle spasm and in severe cases, diarrhoea [1, 35]. "Mitch" develops when strong sunlight strikes a part of the body that is sweating or 
unclean [36], and in general after engaging in tasks that expose one to strong smells, or smoke

$424[1,37]$. Our study found that "Mitch" also affects female reproductive organs when it is

425

426

427 exposed to excessive sunlight, which they refer it to as "Yemahitsen Mitch" (" gynaecologic mitch'”) (Table 2). In general our result agrees with the findings of Ethiopians at home [15] and in diaspora [1] regarding "Mitch" and its treatment. Studies of the leaf extract of $O$. lamiifolium have shown analgesic effects in mice [38] that support its traditional use against Mitch. O. lamiifolium is considered relatively safe and has not demonstrated any sign of acute toxicity up to the dose of $2000 \mathrm{mg} / \mathrm{kg}$ body weight in experimental mice [39].

C. papaya and Z. officinale were the third and the fourth commonly used plants respectively. Several women in this study claimed that papaya softens their birth canal ("uterus") making them healthy and ready for childbirth (Table 3). Moreover, they claimed that consumption of cold papaya would soothe their gastrointestinal tract relieving them from heartburn, gastritis and cramps (Table 2). Animal studies suggest that unlike its abortifacient property at larger dose, normal consumption of ripe papaya during pregnancy may not pose any developmental toxicity and teratogenicity [40].

Although previous studies, also in Ethiopia, showed that pregnant women commonly use ginger for treating NVP $[1,4,5,20,28]$, our study found that it was mainly used for common colds and flu in pregnancy. This could be due to the fact that previous studies involved mainly women in their earlier stages of pregnancy in which NVP is common. Concerning safety, evidences suggest that ginger did not have harmful maternal or neonatal effects $[1,4]$. Its side effects reported in our study were also similar with previous reports [1]. 
447 Several socio-demographic factors were associated with use of medicinal plants in pregnancy

448 (Table 1). We found that women who did not have access to health facility (incl. health posts)

449

450

451

452

453

454

455

456

457

458

459

460

461

462

463

464

465

466

467

468

469

470

471

were seven times more likely to use medicinal plants than their counterparts. This is in line with other studies showing that in Africa people use traditional medicine when facilities are either unavailable or unaffordable $[4,22]$. Similarly, women admitted in maternity ward were three-fold as likely to use medicinal plants as their counterparts. Most women in the maternity ward were in their final stage of pregnancy and might be using more medicinal plants for childbirth than those admitted in gynaecology ward in which hyperemesis and abortions predominate. Similarly, women who used khat or consumed alcohol as well as conventional medicine were twice or more as likely to use medicinal plants as their counterparts, and may either indicate a higher willingness to intake different substances in pregnancy and/or higher morbidity. Since interactions between medicinal plants and conventional medicines may occur and potentially may cause complications $[4,15,41]$, caution with concomitant use should be recommended. Health care personnel at the wards were often not informed; neither involved in decisions nor aware about the women's use of medicinal plants in relation to childbirth. As pregnancy is a time of particular vulnerability, cautious use of medicinal plants is necessary and health-care professionals should ask women about their use and provide them evidencebased information.

Despite the size and extensive data collection, this study has several limitations that should be taken into consideration. Firstly, JUMC is a tertiary referral hospital with a larger proportion of women with pregnancy complications. Our findings may not be representative of women in secondary or primary care. Secondly, as this study was based in southwest Ethiopia, participants were mostly Muslims and from the Oromo ethnic group. These groups had a lower use of medicinal plants in pregnancy compared to participants who were Orthodox and from 
the Dawuro ethnic group. Our results will consequently not be generalizable to the entire country. This finding underpins the importance of including ethnic and religious background information in studies on medical plants, as it will have large impacts on utilization and reporting patterns. Thirdly, data were collected based on pregnant women's self-report and thus depended on their accuracy of recall and reporting as well as willingness to disclose utilization. It may well be that the use of medicinal plants is underestimated due to poor recall or underreporting. This may be especially important during face-to-face interviews for certain medicinal herbs, recreational or illicit drugs that are culturally unacceptable. Actual medicinal plant use in pregnancy may therefore be higher in real life, and/or different in other populations and regions in Ethiopia.

\section{Conclusion}

Almost a third of women at the tertiary hospital in Ethiopia used medicinal plants during pregnancy, most frequently to prepare, induce, reduce the intensity or shorten duration of labour. Seeds and dry plant material was mostly used, sugar the most common excipient and oral route of administration was predominant. The most frequently used medicinal plants were Linum usitatissimum L. (flaxseed- use with caution) (22.0\%), Ocimum lamiifolium L. (damakessie- safety unknown) (3.6\%), and Carica papaya L. (papaya- use with caution) (3.1\%). O. lamiifolium was mainly used for treatment of an illness a culturally common illness in Ethiopia called "Mitch", a febrile illness believed to develop after exposure to excessive sunlight. Few women reported safety concerns regarding medicinal plant use in pregnancy. The most important factors associated with use of medicinal plants in pregnancy were lack of access to health care facilities, hospitalization in the maternity ward and social drug use.

Given that women use unsafe plants during pregnancy, increased awareness about potential benefits or risks of medicinal plants use during pregnancy among health care professionals and 
patients, and increased access to health care facilities are important in order to promote safer pregnancies and better health outcomes for women and their unborn children.

\section{Footnotes}

Acknowledgements: We are grateful to the pregnant women admitted at JUMC who generously shared with us information about their medicinal plant use. The authors owe a debt of gratitude to the enumerators who skilfully collected the data. We would like to thank Norwegian PhD School in Pharmaceutical Sciences for the travel grant assistance for the data collection. Special thanks go to the Norwegian Loan Fund (Lånekassen) for granting scholarship for the PhD student (SMA) in the University of Oslo. The authors are also indebted to Dr. Ibrahimu Mdala for assistance with data analysis.

Contributors: SMA and HN conceived the idea for the study and its design. SMA collected, analysed and interpreted data and drafted the manuscript. YAA and JS participated in study coordination. SMA and HN revised and finalized the manuscript. SMA, HN, JS and YAA critically reviewed the manuscript and contributed intellectual content. All authors read and approved the final manuscript.

Funding: This research received no specific grant from any funding agency in the public, commercial or not-for-profit sectors.

\section{Competing interests: None declared.}

Patient consent for publication: Not required.

Ethics approval: This study was approved by Jimma University Institute of health Institutional Review Board (IRB) (ref. no. IHRPGC 7206/07) in Ethiopia, and Regional Committees for Medical and Health Research Ethics (REK Sør-Øst B) (Ref.no. 2015/2135) in Norway. 
522 Data availability statement: Data are available upon reasonable request.

\section{Supplementary data}

524 Supplementary table 1: Consent form and questionnaire for medicinal plants used during 525 pregnancy at JUMC, Ethiopia

526 Supplementary table 2: Data extraction form for pregnancy characteristics and admission 527 details at JUMC, Ethiopia

528 Supplementary table 3: Definitions of safety categories of medicinal plants used during 529 pregnancy at JUMC, Ethiopia

530 Supplementary table 4: Overview of medicinal plants used during pregnancy according to 531 safety classification and number of users at JUMC, Ethiopia

532 Supplementary table 5: Overview of the utilization pattern of the most frequently used 533 medicinal plants during pregnancy at JUMC, Ethiopia 
535 Table 1. Characteristics of women according to medicinal plant use during pregnancy at JUMC,

536 Ethiopia

\begin{tabular}{|c|c|c|c|c|c|}
\hline \multirow[t]{4}{*}{ Characteristics } & \multirow{4}{*}{$\begin{array}{l}\text { No. }(\%) \\
1117(100)^{a}\end{array}$} & \multicolumn{2}{|c|}{$\begin{array}{l}\text { Medicinal plant use during } \\
\text { pregnancy }\end{array}$} & \multirow{4}{*}{$\begin{array}{l}\text { Crude OR } \\
{[95 \% \mathrm{CI}]^{b}}\end{array}$} & \multirow{4}{*}{$\begin{array}{l}\text { Adjusted OR } \\
{[95 \% \mathrm{CI}]^{\mathrm{c}}}\end{array}$} \\
\hline & & Yes & No & & \\
\hline & & No. $(\%)$ & No. $(\%)$ & & \\
\hline & & $319(28.6)$ & $798(71.4)$ & & \\
\hline \multicolumn{6}{|l|}{ Place of Residence } \\
\hline Urban & $595(53.3)$ & $165(51.7)$ & $430(53.9)$ & 1 & - \\
\hline Rural & $522(46.7)$ & $154(48.3)$ & $368(46.1)$ & 1.09 [0.84-1.41] & \\
\hline \multicolumn{6}{|l|}{ Age (years) ${ }^{d}$} \\
\hline$\leq 20$ & $223(20.0)$ & $52(16.3)$ & $171(21.4)$ & 1 & 1 \\
\hline $21-25$ & $388(34.7)$ & $116(36.4)$ & $272(34.1)$ & $1.40[0.96-2.05]$ & $1.30[0.88-1.94]$ \\
\hline $26-30$ & $320(28.7)$ & $102(32.0)$ & $218(27.3)$ & $1.54[1.04-2.27]$ & $1.42[0.94-2.14]$ \\
\hline$\geq 31$ & $186(16.7)$ & $49(15.4)$ & $137(17.2)$ & $1.18[0.75-1.85]$ & 1.17 [0.73-1.87] \\
\hline \multicolumn{6}{|l|}{ Marital status } \\
\hline Married & $1071(95.9)$ & $314(98.4)$ & 757 (94.9) & 1 & 1 \\
\hline Others ${ }^{\mathrm{e}}$ & $46(4.1)$ & $5(1.6)$ & $41(5.1)$ & $0.29[0.12-0.75]$ & 0.39 [0.14-1.09] \\
\hline \multicolumn{6}{|l|}{ Religion } \\
\hline Islam & $731(65.4)$ & $201(63.0)$ & $530(66.4)$ & 1 & \\
\hline Orthodox & $305(27.3)$ & $99(31.0)$ & $206(25.8)$ & 1.27 [0.95-1.69] & - \\
\hline Protestant/Others $\mathbf{f}$ & $81(7.3)$ & $19(6.0)$ & $62(7.8)$ & 0.81 [0.47-1.39] & \\
\hline \multicolumn{6}{|l|}{ Educational level g } \\
\hline Illiterate & $378(34.0)$ & $98(30.7)$ & $280(35.1)$ & 1 & 1 \\
\hline Primary /read \& write & $470(42.3)$ & $138(43.3)$ & $332(41.6)$ & $1.19[0.88-1.61]$ & $1.22[0.88-1.68]$ \\
\hline Secondary school & $162(14.6)$ & $56(17.6)$ & $106(13.3)$ & $1.51[1.02-2.25]$ & $1.54[1.01-2.36]$ \\
\hline Post-secondary school & $102(9.2)$ & $27(8.5)$ & $75(9.4)$ & $1.03[0.63-1.69]$ & $1.06[0.62-1.79]$ \\
\hline \multicolumn{6}{|l|}{ Occupation } \\
\hline House wife & $524(46.9)$ & $142(44.5)$ & $382(47.9)$ & 1 & - \\
\hline Farmer & $261(23.4)$ & $82(25.7)$ & $179(22.4)$ & $1.23[0.89-1.71]$ & \\
\hline Trader/Merchant & $163(14.6)$ & $49(15.4)$ & $114(14.3)$ & $1.16[0.79-1.70]$ & \\
\hline Government employee & $95(8.5)$ & $30(9.4)$ & $65(8.1)$ & $1.24[0.77-1.99]$ & \\
\hline Others ${ }^{\mathbf{h}}$ & $74(6.6)$ & $16(5.0)$ & $58(7.3)$ & $0.74[0.41-1.33]$ & \\
\hline \multicolumn{6}{|l|}{ Ethnic Group } \\
\hline Oromo & $779(69.7)$ & $224(70.2)$ & $555(69.5)$ & 1 & 1 \\
\hline Amhara & $87(7.8)$ & $21(6.6)$ & $66(8.3)$ & $0.79[0.47-1.32]$ & $0.83[0.48-1.45]$ \\
\hline Yem & $81(7.3)$ & $24(7.5)$ & $57(7.1)$ & $1.04[0.63-1.72]$ & 1.14 [0.66-1.97] \\
\hline Dawuro & $70(6.3)$ & $12(3.8)$ & $58(7.3)$ & $0.51[0.27-0.97]$ & $0.64[0.33-1.25]$ \\
\hline Others $^{\mathbf{i}}$ & $100(9.0)$ & 38 (11.9) & $62(7.8)$ & $1.52[0.99-2.34]$ & $1.57[1.00-2.48]$ \\
\hline \multicolumn{6}{|l|}{ Access to health facility ${ }^{j}$} \\
\hline Yes & $1107(99.1)$ & $313(98.1)$ & $794(99.5)$ & 1 & 1 \\
\hline No & $10(0.9)$ & $6(1.9)$ & $4(0.5)$ & $3.81[1.07-13.58]$ & $6.92[1.77-27.10]$ \\
\hline \multicolumn{6}{|l|}{$\begin{array}{l}\text { Walking distance to the } \\
\text { nearest health facility }\end{array}$} \\
\hline Close, $\leq 30 \mathrm{~min}$. & $731(66.4)$ & $203(63.6)$ & $528(66.2)$ & 1 & - \\
\hline Somewhat far, 31-60 min. & $245(22.3)$ & $67(21.0)$ & $178(22.3)$ & 0.98 [0.71-1.35] & \\
\hline Far, $>60 \mathrm{~min}$ & $125(11.4)$ & $43(13.5)$ & $82(10.3)$ & $1.36[0.91-2.04]$ & \\
\hline \multicolumn{6}{|l|}{ Gravidity $^{k}$} \\
\hline Primigravida & $431(38.6)$ & $307(38.5)$ & $124(38.9)$ & 1 & - \\
\hline Multigravida & $686(61.4)$ & $491(61.5)$ & $195(61.1)$ & $0.98[0.75-1.28]$ & \\
\hline \multicolumn{6}{|l|}{ Gestational age } \\
\hline Preterm pregnancy & $231(20.7)$ & $60(18.8)$ & $171(21.4)$ & 1 & 1 \\
\hline Term pregnancy & $735(65.8)$ & $208(65.2)$ & $527(66.0)$ & $1.13[0.81-1.57]$ & $0.80[0.52-1.25]$ \\
\hline Post term pregnancy & $62(5.6)$ & $27(8.5)$ & $35(4.4)$ & $2.20[1.23-3.93]$ & $1.65[0.85-3.20]$ \\
\hline Others ${ }^{1}$ & $89(8.0)$ & $24(7.5)$ & $65(8.1)$ & $1.05[0.61-1.83]$ & $0.72[0.38-1.36]$ \\
\hline
\end{tabular}

Patient type 


\begin{tabular}{|c|c|c|c|c|c|}
\hline Gynaecology ward & $125(11.2)$ & $22(6.9)$ & $103(12.9)$ & 1 & 1 \\
\hline Maternity ward & $992(88.8)$ & $297(93.1)$ & $695(87.1)$ & $2.00[1.24-3.23]$ & $2.80[1.43-5.48]$ \\
\hline \multicolumn{6}{|l|}{ Chronic illness $^{\mathrm{m}}$} \\
\hline No & $1061(95.0)$ & $294(92.2)$ & $767(96.1)$ & 1 & 1 \\
\hline Yes & $56(5.0)$ & $25(7.8)$ & $31(3.9)$ & $2.10[1.22-3.62]$ & $1.83[1.04-3.24]$ \\
\hline \multicolumn{6}{|c|}{ Conventional medicine use $^{n}$} \\
\hline No & $817(73.1)$ & $209(65.5)$ & $608(76.2)$ & 1 & 1 \\
\hline Yes & $300(26.9)$ & $110(34.5)$ & $190(23.8)$ & $1.68[1.27-2.23]$ & $1.83[1.36-2.46]$ \\
\hline \multicolumn{6}{|c|}{ Chew Khat (Catha edulis) ${ }^{\circ}$} \\
\hline No & $1052(94.2)$ & $289(90.6)$ & $763(95.6)$ & 1 & 1 \\
\hline Yes & $65(5.8)$ & $30(9.4)$ & $35(4.4)$ & $2.26[1.36-3.75]$ & $2.53[1.46-4.39]$ \\
\hline \multicolumn{6}{|l|}{ Alcohol consumption } \\
\hline No & $1071(95.9)$ & $297(93.1)$ & $774(97.0)$ & 1 & 1 \\
\hline Yes & $46(4.1)$ & $22(6.9)$ & $24(3.0)$ & $2.39[1.32-4.33]$ & $2.43[1.28-4.62]$ \\
\hline \multicolumn{6}{|c|}{$\begin{array}{l}\text { Past adverse pregnancy } \\
\text { outcome }\end{array}$} \\
\hline No/not applicable & $994(89.0)$ & $275(86.2)$ & $719(90.1)$ & 1 & - \\
\hline Yes & $123(11.0)$ & $44(13.8)$ & 79 (9.9) & $1.51[1.00-2.28]$ & \\
\hline
\end{tabular}

${ }^{a}$ Numbers may not add up to 1117 due to missing values, ${ }^{\text {b }} \mathrm{CI}$, confidence interval, OR, odds ratio; Significant findings are in bold $(\mathrm{P}<0.05)$; ${ }^{\mathrm{c} A d j u s t e d ~ f o r ~ a g e, ~ m a r i t a l ~ s t a t u s, ~ e d u c a t i o n a l ~ l e v e l, ~ e t h n i c ~ g r o u p, ~ a c c e s s ~ t o ~ h e a l t h ~ f a c i l i t y, ~ g e s t a t i o n a l ~ a g e, ~}$ patient type, chronic illness, conventional medicine use, chew khat, alcohol consumption; ${ }^{\mathrm{d}}$ Median age 25 years, interquartile range $22-30$ years; ${ }^{\mathrm{e}}$ Others includes single $41(3.7 \%)$, divorced $4(0.4 \%)$, widowed $1(0.1 \%)$; ${ }^{\text {Protestant/Others }}$ includes Protestant 74(6.6), Catholic 2(0.2\%), Waqqefeta 1(0.1\%), missing 4(0.4); gRead \& write: no formal education but can read and write due to literacy campaigns, traditional religious institution and informal peer learning, Primary school: Grade 1-8, Secondary school: Grade 9-12; Post-secondary school: Technical and vocational school, college or university; ${ }^{\mathrm{h}}$ Others includes daily labourers $24(2.1)$, students $22(2.0)$, private institution workers $18(1.6)$, other sectors $10(0.9 \%)$; ${ }^{i}$ Others includes Gurage 41(3.7), Silte 30(2.7), Kaffa 16(1.4), Tigre 3(0.3), Wolayita 3(0.3), mixed ethnic backgrounds 7(0.6); ${ }^{\mathrm{j} A c c e s s}$ to health facility means access to either primary, secondary or tertiary levels of healthcare; it mainly represents access to health posts; ${ }^{\mathrm{k}}$ Gravidity includes the current pregnancy; ${ }^{\mathrm{l} W o m e n}$ are in the first, second or third trimester of pregnancy but exact week of pregnancy is not known ; mIncludes hypertension, diabetes mellitus, asthma, cardiac diseases,

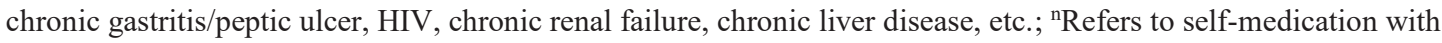
conventional medicine before hospitalization; ${ }^{\circ}$ Khat (Catha edulis) plant leaves are chewed by people for their stimulant action 


\begin{tabular}{|c|c|c|}
\hline Variables & Number $(\%)^{a}$ & Most common medicinal plants (number of users) \\
\hline \multirow[t]{3}{*}{ Induction and "reduction" of labour ${ }^{b}$} & $192(60.2)$ & Linum usitatissimum (Flaxseed) (179) \\
\hline & & Trigonella foenum-graecum (Fenugreek) (6) \\
\hline & & Carica papaya (Papaya) (4) \\
\hline \multirow[t]{3}{*}{ Common cold/flu } & $65(20.4)$ & Zingiber officinale (Ginger) (23) \\
\hline & & Allium sativum (Garlic) (13) \\
\hline & & Eucalyptus globulus (Nech-bahir zaf ) (12) \\
\hline \multirow[t]{3}{*}{ Preparation for labour } & $50(15.7)$ & Linum usitatissimum (Flaxseed) (22) \\
\hline & & Carica papaya (Papaya) (17) \\
\hline & & Trigonella foenum-graecum (Fenugreek) (11) \\
\hline \multirow[t]{3}{*}{ Abdominal cramps/ache } & $30(9.4)$ & Nigella sativa (Black seed) (10) \\
\hline & & Allium sativum (Garlic) (5) \\
\hline & & Carica papaya (Papaya) (4) \\
\hline \multirow[t]{3}{*}{ Headache/Migraine } & $27(8.5)$ & Nigella sativa (Black seed) (10) \\
\hline & & Ocimum lamiifolium (Damakessie) (8) \\
\hline & & Allium sativum (Garlic) (3) \\
\hline \multirow[t]{2}{*}{ Heartburn/reflux problems } & $27(8.5)$ & Linum usitatissimum (Flaxseed) (16) \\
\hline & & Carica papaya (Papaya) (5) \\
\hline Mitch $^{\mathrm{c}}$ & $24(7.5)$ & Ocimum lamiifolium (Damakessie) (18) \\
\hline Gastritis/burning sensation & $22(6.9)$ & Linum usitatissimum (Flaxseed) (19) \\
\hline Constipation/obstipation & $17(5.3)$ & Linum usitatissimum (Flaxseed) (16) \\
\hline \multirow[t]{2}{*}{ General wellbeing } & $15(4.7)$ & Allium sativum (Garlic) (5) \\
\hline & & Ruta chalepensis (Fringed rue) (3) \\
\hline \multirow[t]{2}{*}{ Nausea } & $11(3.4)$ & Zingiber officinale (Ginger) (4) \\
\hline & & Ruta chalepensis (Fringed rue) (4) \\
\hline \multirow[t]{2}{*}{ Helminths } & $6(1.9)$ & Carica papaya (Papaya) (2) \\
\hline & & Hagenia abyssinica (Kosso) (2) \\
\hline \multirow[t]{5}{*}{ Leg/foot Swelling } & $5(1.6)$ & Linum usitatissimum (Flaxseed) (1) \\
\hline & & Cinnamomum verum (Cinnamon) (1) \\
\hline & & Croton macrostachyus (Bisena) (1) \\
\hline & & Veronia amygdalina (Grawa) (1) \\
\hline & & B'auu (1) \\
\hline Prevent bad smell & $5(1.6)$ & Ocimum lamiifolium (Damakessie) (5) \\
\hline \multirow[t]{5}{*}{ Strong craving } & $5(1.6)$ & Linum usitatissimum (Flaxseed) (1) \\
\hline & & Carica papaya (Papaya) (1) \\
\hline & & Nigella sativa (Black seed) (1) \\
\hline & & Ruta chalepensis (Fringed rue) (1) \\
\hline & & Zingiber officinale (Ginger) (1) \\
\hline Emergency illnesses & $4(1.3)$ & Ocimum lamiifolium (Damakessie) (3) \\
\hline Postpartum bathing & $4(1.3)$ & Eucalyptus globulus (Nech-bahir zaf) (3) \\
\hline Vomiting & $3(0.9)$ & Zingiber officinale (Ginger) (2) \\
\hline Yemahitsen mitch ${ }^{c}$ & $3(0.9)$ & Croton macrostachyus (Bisena) (1) \\
\hline \multirow[t]{2}{*}{ ('gynaecologic mitch') } & & Ocimum lamiifolium (Damakessie) (1) \\
\hline & & Pycnostachys abyssinica (Yeroo) (1) \\
\hline \multirow[t]{3}{*}{ Depression } & $3(0.9)$ & Echinops kebericho (Kebericho) (1) \\
\hline & & Ruta chalepensis (Fringed rue) (1) \\
\hline & & Cinnamomum verum (Cinnamon) (1) \\
\hline \multirow[t]{2}{*}{ Wellbeing and nourishing the foetus } & $3(0.9)$ & Linum usitatissimum (Flaxseed) (2) \\
\hline & & Trigonella foenum-graecum (Fenugreek) (1) \\
\hline \multirow[t]{2}{*}{ Cough } & $2(0.6)$ & Nigella sativa (Black seed) (1) \\
\hline & & Saccharum officinarum (Sugar crystals) (1) \\
\hline \multirow[t]{2}{*}{ Birdd ${ }^{d}$} & $2(0.6)$ & Allium sativum (Garlic) (1) \\
\hline & & Nigella sativa (Black seed) (1) \\
\hline \multirow[t]{2}{*}{ Diarrhoea } & $2(0.6)$ & Ocimum lamiifolium (Damakessie) (1) \\
\hline & & Taverniera abyssinica (Dingetegn) (1) \\
\hline Joint pain (kurtimatt) & $2(0.6)$ & Allium sativum (Garlic) (1) \\
\hline
\end{tabular}




\begin{tabular}{|c|c|c|}
\hline Variables & Number $(\%)^{a}$ & Most common medicinal plants (number of users) \\
\hline & & Nigella sativa (Black seed) (1) \\
\hline Sleeping problems & $2(0.6)$ & Artemisia abyssinica (Chikugn) (2) \\
\hline Mental wellbeing & $2(0.6)$ & Ruta chalepensis (Fringed rue) (2) \\
\hline Evil eye & $2(0.6)$ & $\begin{array}{l}\text { Artemisia afra }(\text { Ariti })(1) \\
\text { Veronia amygdalina }(\text { Grawa })(1)\end{array}$ \\
\hline Others ${ }^{\mathrm{e}}$ & $15(4.7)$ & $\begin{array}{l}\text { Linum usitatissimum (Flaxseed) (3) } \\
\text { Allium sativum (Garlic) (3) } \\
\text { Ocimum lamiifolium (Damakessie) (3) }\end{array}$ \\
\hline
\end{tabular}

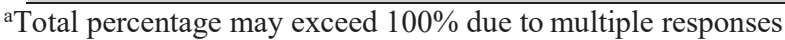

556 beduction of labour: Includes reduced intensity and shortened duration of labour.

557 'Mitch': A febrile illness believed to develop when strong sunlight strikes a part of the body that is sweating or unclean.

558 d'Birdd': An illness typified by a feeling of chills, arthralgia, myalgia, generalized body weakness, pain (particularly chest pain) and coughing. In general, it is characterized by pneumonia/flu-like symptoms.

560 'Others includes make labour simple, stomach rambling, quicken labour, prevent 'megagna', fever, facilitation of digestion, tonsillitis, pregnancy associated body/physical illnesses, skin rashes ('Shifta'), abdominal distension/bloating, throat congestion ('Guroroyen siyafinegn'), malaria, appetizer, upper extremity fatigability, for any illness, each with a frequency of one. 

number of users and the most common indications at JUMC, Ethiopia

\begin{tabular}{|c|c|c|}
\hline $\begin{array}{l}\text { Medicinal plant (English name) } \\
\text { (local name) Preparation method }\end{array}$ & $\begin{array}{l}\text { Number of users } \\
(\mathrm{n}=\mathbf{3 1 9}), \mathrm{n}(\mathbf{2 8 . 6 \% )}\end{array}$ & $\begin{array}{l}\text { Most common indications } \\
\text { (No. of citations) }\end{array}$ \\
\hline Linum usitatissimum L. (Flaxseed or Linseed) (Telba) & $246(77.1)$ & $\begin{array}{l}\text { Induction or "reduction" of labour } \\
(179)^{\text {a }}\end{array}$ \\
\hline $\begin{array}{l}\text { Flax seeds are roasted, pounded, thoroughly mixed with } \\
\text { water and consumed in soup form. }\end{array}$ & & $\begin{array}{l}\text { Prepare for labour (22) } \\
\text { Heartburn/reflux problems (19) } \\
\text { Constipation/obstipation (16) } \\
\text { Gastritis/burning sensation (14) } \\
\text { Abdominal cramps or ache (2) }\end{array}$ \\
\hline $\begin{array}{l}\text { Ocimum lamiifolium L. (No common English name) } \\
\text { (Damakessie) } \\
\text { Adding minced fresh leaves or steeping in leaves in tea, } \\
\text { coffee, milk or decoction or maceration of minced root are } \\
\text { drunk, or fresh leaves are put in nostrils and sniffed }\end{array}$ & $40(12.5)$ & $\begin{array}{l}\text { Mitch (19) }{ }^{\mathrm{b}} \\
\text { Common cold/flu (10) } \\
\text { Headaches/Migraine (8) } \\
\text { Prevent bad smell (5) } \\
\text { Emergency cases/illnesses (3) } \\
\text { Nausea (2) }\end{array}$ \\
\hline $\begin{array}{l}\text { Carica papaya L. (Papaya) (Papaya) } \\
\text { Ripened fresh fruit is eaten or its juice is extracted and then } \\
\text { drunk }\end{array}$ & $35(11.0)$ & $\begin{array}{l}\text { Prepare for labour (17) } \\
\text { Heartburn/reflux problems (5) } \\
\text { Induce labour (4) } \\
\text { Abdominal cramps or ache (4) } \\
\text { Gastritis/burning sensation (3) } \\
\text { Helminths (2) }\end{array}$ \\
\hline Zingiber officinale Roscoe. (Ginger) (Zingibil) & $29(9.1)$ & $\begin{array}{l}\text { Common cold/flu (23) } \\
\text { Nausea (4) }\end{array}$ \\
\hline $\begin{array}{l}\text { Drinking aqueous decoction or maceration, or drinking } \\
\text { minced root with water or tea }\end{array}$ & & $\begin{array}{l}\text { Vomiting (2) } \\
\text { Abdominal cramps (2) }\end{array}$ \\
\hline Allium sativum L. (Garlic) (Nech shinkrut) & $28(8.8)$ & $\begin{array}{l}\text { Common cold/flu (13) } \\
\text { General wellbeing (5) }\end{array}$ \\
\hline $\begin{array}{l}\text { Drinking minced cloves with tea, coffee, milk or eating raw } \\
\text { cloves with Ethiopian bread, 'Injera' }\end{array}$ & & $\begin{array}{l}\text { Abdominal cramps (5) } \\
\text { Headaches/Migraine (3) } \\
\text { Birdd (2) }\end{array}$ \\
\hline $\begin{array}{l}\text { Trigonella foenum-graecum L. (Fenugreek) (Abish) } \\
\text { Drink aqueous maceration of seeds or consume roasted and } \\
\text { powdered seeds in soup form }\end{array}$ & $24(7.5)$ & $\begin{array}{l}\text { Prepare for labour (11) } \\
\text { Induce labour (6) } \\
\text { Gastritis/burning sensation (4) }\end{array}$ \\
\hline $\begin{array}{l}\text { Nigella sativa L (Black seed) (Tikur Azmud) } \\
\text { Drinking few drops of the seed oil with tea, coffee, milk or } \\
\text { sniffing the oil drops }\end{array}$ & $21(6.4)$ & $\begin{array}{l}\text { Headaches/Migraine (10) } \\
\text { Abdominal cramps or ache }(7) \\
\text { Common cold/flu (6) }\end{array}$ \\
\hline Ruta chalepensis L. (Fringed rue) (Tenadam) & $15(4.7)$ & $\begin{array}{l}\text { Nausea (4) } \\
\text { General wellbeing (3) }\end{array}$ \\
\hline $\begin{array}{l}\text { Adding minced fresh leaves or steeping in leaves in tea, } \\
\text { coffee, or milk and then drunk or fresh leaves squeezed, } \\
\text { and then drunk }\end{array}$ & & $\begin{array}{l}\text { Headaches/Migraine (2) } \\
\text { Mental wellbeing (2) } \\
\text { Abdominal cramps (2) }\end{array}$ \\
\hline $\begin{array}{l}\text { Eucalyptus globulus Labill. (“Eucalyptus”) (Nech-bahir } \\
\text { zaf) } \\
\text { Leaves are boiled in water, patient fumigated and the } \\
\text { vapour is inhaled }\end{array}$ & $13(4.1)$ & $\begin{array}{l}\text { Common cold/flu (12) } \\
\text { Postpartum bathing ( } 3) \\
\text { Mitch (2) }\end{array}$ \\
\hline \multicolumn{3}{|c|}{$\begin{array}{l}\text { a' Reduction of labour': Includes reduced intensity and shortened duration of labour; 'Mitch': A febrile illness believed to } \\
\text { develop when strong sunlight strikes a part of the body that is sweating or unclean; 'Birdd': An illness typified by a feeling } \\
\text { of chills, arthralgia, myalgia, generalized body weakness, pain (particularly chest pain) and coughing. In general, it is } \\
\text { characterized by pneumonia/flu-like symptoms }\end{array}$} \\
\hline
\end{tabular}


571 Table 4. Pregnant women's self-reported safety concerns and experiences with medicinal plants at

572 JUMC, Ethiopia, n=319

\begin{tabular}{|c|c|c|c|}
\hline $\begin{array}{l}\text { Medicinal plants (MPs), reported } \\
\text { reasons for contraindication (No. of } \\
\text { citations) }\end{array}$ & $\begin{array}{l}\text { MPs, reported precautions (No. } \\
\text { of citations) }\end{array}$ & $\begin{array}{l}\text { MPs, reported Side } \\
\text { effects (No. of } \\
\text { citations) }\end{array}$ & $\begin{array}{l}\text { MPs, reported adverse } \\
\text { drug reactions (No. of } \\
\text { citations) }\end{array}$ \\
\hline $\begin{array}{l}\text { Flaxseed: Cause uterine contractions, } \\
\text { miscarriage or premature labour (2), } \\
\text { Dries up breastmilk (1) }\end{array}$ & $\begin{array}{l}\text { Flaxseed }{ }^{\text {a: }} \text { Aloe vera is bitter and } \\
\text { makes flaxseed preparation } \\
\text { unpalatable (1), the woman } \\
\text { should stay away from sunlight } \\
\text { while /after taking flaxseed } \\
\text { preparation (1) }\end{array}$ & $\begin{array}{l}\text { Flaxseed: Nausea (4), } \\
\text { Postpartum shivering } \\
\text { (1), } \\
\text { Diarrhoea (1), } \\
\text { Vomiting (1), Loss of } \\
\text { appetite (1), }\end{array}$ & $\begin{array}{l}\text { Flaxseed: Severe } \\
\text { postpartum shivering } \\
\text { (1), Loss of } \\
\text { consciousness (1), } \\
\text { Uterine rupture (1), Still } \\
\text { birth (1) }\end{array}$ \\
\hline Kosso $^{b}:$ Causes uterine stimulation (4) & $\begin{array}{l}\text { Kosso: Kosso protects the mother } \\
\text { from outside person's 'tila' as it } \\
\text { may kill her (1), After taking } \\
\text { Kosso, the woman should stay at } \\
\text { home for } 2 \text { days protected from } \\
\text { outside person's 'tila' (1) }\end{array}$ & Kosso: Diarrhoea (2) & $\begin{array}{l}\text { Kosso: Severe diarrhoea } \\
\text { (2) }\end{array}$ \\
\hline $\begin{array}{l}\text { Dingetegna: harmful during pregnancy, } \\
\text { reason unknown }(1)^{\mathrm{d}}\end{array}$ & $\begin{array}{l}\text { Dingetegna }{ }^{\mathrm{e}} \text { : the woman who } \\
\text { took dingetegna should stay at } \\
\text { home, outsiders should not be } \\
\text { allowed to get in for fear of their } \\
\text { 'tila' (1) }\end{array}$ & $\begin{array}{l}\text { Dingetegna: } \\
\text { Diarrhoea (2), } \\
\text { Vomiting (1) }\end{array}$ & $\begin{array}{l}\text { Dingetegna: } \\
\text { Severe diarrhoea (2) }\end{array}$ \\
\hline $\begin{array}{l}\text { Damakessie: Causes uterine stimulation } \\
\text { (1) }\end{array}$ & $\begin{array}{l}\text { Damakessie }^{f} \text { : After applying MPs } \\
\text { stay at home, going outside is } \\
\text { forbidden }(1)^{\mathrm{g}}\end{array}$ & $\begin{array}{l}\text { Damakessie: Loss of } \\
\text { appetite (1), Bitter } \\
\text { (after) taste (1), } \\
\text { Sneezing (1) }\end{array}$ & $\begin{array}{l}\text { Chikugn }{ }^{\mathrm{h}} \text { : } \\
\text { Anencephaly: giving } \\
\text { birth to a headless } \\
\text { neonate (1) }\end{array}$ \\
\hline $\begin{array}{l}\text { Tej Sar }{ }^{i} \text { : Causes uterine stimulation } \\
\text { (1), harmful during pregnancy, reason } \\
\text { unknown (1) }\end{array}$ & $\begin{array}{l}\text { Cinnamon }{ }^{\mathrm{j}} \text { : inflammatory to the } \\
\text { stomach, thus eat food before } \\
\text { taking cinnamon (1) }\end{array}$ & $\begin{array}{l}\text { Cinnamon: Heart burn } \\
\text { (1) }\end{array}$ & $\begin{array}{l}\text { Cinnamon: Severe heart } \\
\text { burn (1) }\end{array}$ \\
\hline $\begin{array}{l}\text { Ensilal k: Causes uterine stimulation } \\
\text { (1), harmful during pregnancy, reason } \\
\text { unknown (1) }\end{array}$ & $\begin{array}{l}\text { Garlic: larger dose of garlic is } \\
\text { irritant, thus eat food before } \\
\text { taking it (1) }\end{array}$ & $\begin{array}{l}\text { Garlic: Discomfort to } \\
\text { foetus (1), Gastric } \\
\text { irritation (1), Loss of } \\
\text { appetite (1) }\end{array}$ & $\begin{array}{l}\text { Garlic: Harm to the } \\
\text { foetus (1), Bad mouth } \\
\text { smell (1), Severe heart } \\
\text { burn (1) }\end{array}$ \\
\hline $\begin{array}{l}\left.\text { Grawa }{ }^{\mathrm{l}} \text { : Causes uterine stimulation ( } 2\right) \\
\text { harmful during pregnancy, reason } \\
\text { unknown }(1)^{\mathrm{d}}\end{array}$ & $\begin{array}{l}\text { Ginger: stomach irritant, thus eat } \\
\text { food before taking ginger ( } 1 \text {, } \\
\text { beware since ginger decreases } \\
\text { appetite (1) and induces fever (1) }\end{array}$ & $\begin{array}{l}\text { Ginger: Gastric } \\
\text { irritation (2), Heart } \\
\text { burn (2), Discomfort } \\
\text { to foetus (1) }\end{array}$ & $\begin{array}{l}\text { Ginger: Harm to foetus } \\
\text { (1), Severe heart burn } \\
\text { (1) }\end{array}$ \\
\hline $\begin{array}{l}\text { Black seed: Generally not good for the } \\
\text { woman and the foetus, thus better not to } \\
\text { take it during pregnancy (1) }\end{array}$ & $\begin{array}{l}\text { Yeroo }^{\mathrm{m}} \text { : After applying MPs stay } \\
\text { at home, going outside is } \\
\text { forbidden }(1)^{\mathrm{g}}\end{array}$ & $\begin{array}{l}\text { Black seed: Gastric } \\
\text { irritation ( } 3) \text {, Loss of } \\
\text { appetite (1) }\end{array}$ & ------ \\
\hline 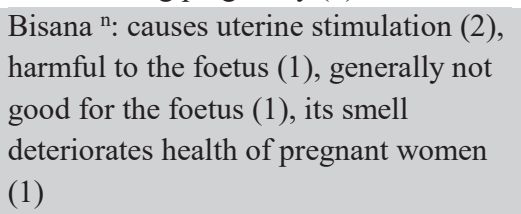 & $\begin{array}{l}\text { Bisana }^{\circ} \text { : After applying MPs stay } \\
\text { at home, going outside is } \\
\text { forbidden }(1)^{\mathrm{g}}\end{array}$ & $\begin{array}{l}\text { "Eucalyptus" p: } \\
\text { Decrease in appetite } \\
\text { (1) }\end{array}$ & ------ \\
\hline $\begin{array}{l}\text { Kebericho: Generally not good for the } \\
\text { foetus(1) }\end{array}$ & $\begin{array}{l}\text { Kebericho }{ }^{\mathrm{q}} \text { : Kebericho interacts } \\
\text { with damakessie and worsen the } \\
\text { Mitch }^{\mathrm{r}} \text { disease (1) }\end{array}$ & $\begin{array}{l}\text { Sugar s: Eye irritation } \\
\text { (1) }\end{array}$ & ------ \\
\hline 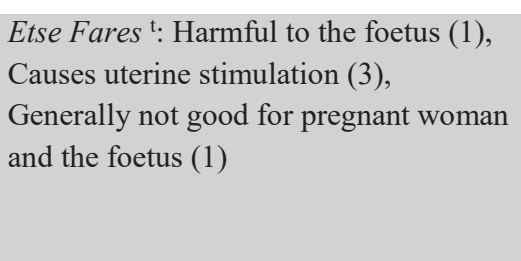 & $\begin{array}{l}\text { Fringed rue: Kebericho should } \\
\text { not be taken with rue because it } \\
\text { will worsen the nausea (1), } \\
\text { beware since it induces fever (1), } \\
\text { There is a plant interacting with } \\
\text { rue, but forgot its name (1) }\end{array}$ & $\begin{array}{l}\text { Fringed rue: Loss of } \\
\text { appetite (1) }\end{array}$ & ------ \\
\hline $\begin{array}{l}\text { 'Baruda' plant }{ }^{\text {u: }} \text { Causes uterine } \\
\text { stimulation (1) }\end{array}$ & ------ & ------ & ------ \\
\hline
\end{tabular}


Endod v: Causes uterine stimulation (1)

Metere $^{w}$ : Harmful to the foetus (1)

Feto $^{\mathrm{x}}$ : Causes uterine stimulation (1)

${ }^{a}$ After consuming Flaxseed (L. usitatissimum) preparation the woman should stay at home, exposure to sunlight results in Mitch disease, ${ }^{\mathrm{b}} \mathrm{H}$. abyssinica,${ }^{\mathrm{c}}$ 'Tila', loosely translated means the shade of a person that is believed to have pernicious effect, ${ }^{\mathrm{d}}$ The plant is harmful during pregnancy, but the woman does not know the reason for contraindication, ${ }^{\mathrm{e}} T$. abyssinica, ${ }^{\mathrm{g}}$ Stay at home after applying the ${ }^{f}$ Damakessie (O. lamiifolium) / ${ }^{\mathrm{m}}$ Yeroo (P. abyssinica) / ${ }^{\circ}$ Bisana (C. macrostachyus) mixture formulation, going outside is forbidden; Otherwise there is relapse of the disease; ${ }^{\mathrm{h}}$ A. abyssinica, ${ }^{\mathrm{i}}$ Cymbopogon citratus (DC.) Stapf, ${ }^{\mathrm{j} C}$. verum, ${ }^{k} \mathrm{~F}$. vulgare, ${ }^{1} \mathrm{~V}$. amygdalina, ${ }^{\mathrm{n}} \mathrm{C}$. macrostachyus, ${ }^{p}$ E.globulus, ${ }^{\mathrm{q}}$ E. kebericho, ${ }^{\mathrm{r}}$ Mitch: A febrile illness believed to develop when strong sunlight strikes a part of the body that is sweating or unclean; ${ }^{\mathrm{s}}$ Saccharum officinarum L., ${ }^{\mathrm{t}}$ Datura stramonium L., "inserting the root in to the vagina and/or drink its juice, ${ }^{\mathrm{v} P h y t o l a c c a}$ dodecandra L'Hér., ${ }^{w}$ Glinus lotoides L., ${ }^{\mathrm{x}}$ L. sativum, ${ }^{\mathrm{b}, \mathrm{x}}$ (particularly use of Kosso with Feto causes severe uterine stimulation).

\section{References}

1. Hailemeskel B, Fullas F, Habte A, Al-Matari R, Brewer D. A review of natural remedies commonly used by Ethiopian immigrants in the USA. Curr Res Integr Med. $2017 ; 2: 31-36$.

2. Jamshidi-Kia F, Lorigooini Z, Amini-Khoei H: Medicinal plants: Past history and future perspective. J Herbmed Pharmacol. 2018; 7:1-7.

3. Mazzari ALDA, Prieto JM: Herbal medicines in Brazil: pharmacokinetic profile and potential herb-drug interactions. Front Pharmacol. 2014; 5: 162.

4. Ahmed SM, Nordeng H, Sundby J, Aragaw YA, de Boer HJ: The use of medicinal plants by pregnant women in Africa: A systematic review. J Ethnopharmacol. 2018; 224:297-313.

5. Shewamene Z, Dune T, Smith CA: The use of traditional medicine in maternity care among African women in Africa and the diaspora: a systematic review. BMC Complement Altern Med. 2017; 17: 382.

6. United States Central Intellingence Agency (CIA). The World Fact Book, Africa: Ethiopia. https://www.cia.gov/the-world-factbook/countries/ethiopia/\#people-andsociety. (2018). Accessed 13 Feb 2019. 
600

601

602

603

604

605

606

607

608

609

610

611

612

613

614

615

616

617

618

619

620

621

622

623

7. Ficquet E. Interfaith Relations between Christianity and Islam in Ethiopia https://www.sciencespo.fr/ceri/fr/oir/interfaith-relations-between-christianity-andislam-ethiopia. (2019). Accessed 20 Feb 2021.

8. Ethiopian Public Health Institute (EPHI). Traditional and Modern Medicine Research http://www.ephi.gov.et/index.php/research/traditional-modern-medicine. (2019). Accessed 25 Feb 2019.

9. Laelago T, Yohannes T, Lemango F: Prevalence of herbal medicine use and associated factors among pregnant women attending antenatal care at public health facilities in Hossana town, southern Ethiopia: facility based cross sectional study. Arch Public Health. 2016; 74 : 7.

10. United Nations Children's Fund (UNICEF). Maternal and Newborn Health Disparities country profiles. https://data.unicef.org/resources/maternal-newborn-healthdisparities-country-profiles/ (2018). Accessed 05 Sept 2019.

11. Ahmed SM, Sundby J, Aragaw YA, Abebe F. Self-Medication and Safety Profile of Medicines Used among Pregnant Women in a Tertiary Teaching Hospital in Jimma, Ethiopia: A Cross-Sectional Study. Int J Environ Res Public Health. 2020; 17: 3993.

12. Bayisa B, Tatiparthi R, Mulisa E: Use of herbal medicine among pregnant women on antenatal care at Nekemte Hospital, Western Ethiopia. Jundishapur J Nat Pharm Prod. 2014; 9: e17368.

13. Jambo A, Mengistu G, Sisay M, Amare F, Edessa D: Self-Medication and Contributing Factors Among Pregnant Women Attending Antenatal Care at Public Hospitals of Harar Town, Ethiopia. Front Pharmacol. 2018; 9: 1063.

14. Mekuria AB, Erku DA, Gebresillassie BM, Birru EM, Tizazu B, Ahmedin A: Prevalence and associated factors of herbal medicine use among pregnant women on 
antenatal care follow-up at University of Gondar referral and teaching hospital, Ethiopia: a cross-sectional study. BMC Complement Altern Med. 2017; 17: 86.

15. Nega SS, Bekele HM, Meles GG, Nordeng H: Medicinal Plants and Concomitant Use with Pharmaceutical Drugs Among Pregnant Women. J Altern Complement Med. $2019 ; 25: 427-434$

16. Hall HG, Griffiths DL, McKenna LG: The use of complementary and alternative medicine by pregnant women: A literature review. Midwifery. 2011; 27: 817-824.

17. Westfall RE: Herbal healing in pregnancy: women's experiences. J Herb Pharmacother. 2003, 3: 17-39.

18. Kennedy DA, Lupattelli A, Koren G, Nordeng H: Herbal medicine use in pregnancy: results of a multinational study. BMC Complement Altern Med. 2013; 13: 355.

19. Ahmed M, Hwang JH, Choi S, Han D: Safety classification of herbal medicines used among pregnant women in Asian countries: a systematic review. BMC Complement Altern Med. 2017; 17: 489.

20. Ahmed M, Hwang JH, Hasan MA, Han D: Herbal medicine use by pregnant women in Bangladesh: a cross-sectional study. BMC Complement Altern Med. 2018; 18: 333.

21. Kennedy DA, Lupattelli A, Koren G, Nordeng H: Safety classification of herbal medicines used in pregnancy in a multinational study. BMC Complement Altern Med. 2016; 16: 102 .

22. Godlove M: Prevalence of herbal medicine use and associated factors among pregnant women attending antenatal clinic at Mbeya Refferal Hospital in 2010 (M.Sc. Thesis) http://hdl.handle.net/123456789/41. Muhimbili University of Health and Allied Sciences, Dar es salaam, Tanzania. (2011). Accessed 03 Oct 2018. 
23. Segni H, Ayana D, Jarso H: Prevalence of Hyperemesis Gravidarum and Associated Factors Among Pregnant Women at Jimma University Medical Center, South West Ethiopia: A Cross-Sectional Study. EC Gynaecol. 2016; 3: 376-387.

24. Jimma University Specialized hospital. http://www.ju.edu.et/jimma-universityspecialized-hospital-jush (2017). Accessed 13 Sept 2018.

25. Kish L: Survey Sampling. New York, NY,USA: John Wiley \& Sons, Inc.; 1965.

26. Mureyi DD, Monera TG, Maponga CC: Prevalence and patterns of prenatal use of traditional medicine among women at selected harare clinics: a cross-sectional study. BMC Complement Altern Med. 2012; 12: 164.

27. Mkize GT: An Assessment of Use of Traditional Medicine in Pregnancy \& Associated Factors Among Black South African Women Delivering in Bertha Gxowa Hospital (M.Sc. Thesis). University of the Witwatersrand, Johannesburg, South Africa. http://wiredspace.wits.ac.za/handle/10539/17340?show=full (2015). Accessed 25 Apr, 2019.

28. Gall A, Shenkute Z. Ethiopian Traditional and Herbal Medications and Their Interactions with Conventional Drugs. https://ethnomed.org/clinical/pharmacy/ethiopian-herb-drug-interactions. Accessed 17 Oct 2018 .

29. Gedif T, Hahn H-J: The use of medicinal plants in self-care in rural central Ethiopia. J Ethnopharmacol. 2003; 87:155-161.

30. Mills E, Duguoa J-J, Perri D, Koren G: Herbal medicines in pregnancy and lactation: An evidence-based approach. London ; New York: Taylor \& Francis; 2006.

31. Mills S, Bone K: The Essential Guide to Herbal Safety. St. Louis, Mo: Elsevier Churchill Livingstone; 2005. 
671 32. Gardner Z, McGuffin M: American Herbal Products Association. American herbal

672

673

674

products association's botanical safety handbook, 2nd edn. Boca Raton, FL: CRC Press; 2013.

33. Zamawe C, King C, Jennings HM, Mandiwa C, Fottrell E: Effectiveness and safety of herbal medicines for induction of labour: a systematic review and meta-analysis. BMJ Open. 2018; 8: e022499.

34. Gokhale S, Sahu A: Pharmacological properties of flaxseed, Linum usitatissimum Linn., as a potential medicinal plant: An overview. World J Pharm Sci. 2016; 4: 207 215.

35. Hodes RM, Teferedegne B: Traditional beliefs and disease practices of Ethiopian Jews. Isr J Med Sci. 1996; 32: 561-7.

36. Hodes R: Cross-cultural medicine and diverse health beliefs. Ethiopians abroad. West J Med. 1997; 166: 29-36.

37. Kifle H, Woldu AM, Asres K, Mazumder A, Bucar F: Composition, antimicrobial and free-radical scavenging properties of the essential oil of Damakese (Ocimum lamiifolium): A popular home remedy in Ethiopia. Int J Essen Oil Ther. 2007; 1: 110 116.

38. Debella A, Makonnen E, Abebe D, Teka F, Kidanemariam AT: Pain management in mice using the aqueous and ethanol extracts of four medicinal plants. East Afr Med J. 2003; 80: 435-439.

39. Kefe A, Giday M, Mamo H, Erko B: Antimalarial properties of crude extracts of seeds of Brucea antidysenterica and leaves of Ocimum lamiifolium. BMC Complement Altern Med. 2016; 16:118. 
694

695

696

697

698

699

700

40. Oderinde O, Noronha C, Oremosu A, Kusemiju T, Okanlawon OA: Abortifacient properties of aqueous extract of Carica papaya (Linn) seeds on female SpragueDawley rats. Niger Postgrad Med J. 2002; 9: 95-8.

41. El Hajj M, Holst L: Herbal Medicine Use During Pregnancy: A Review of the Literature With a Special Focus on Sub-Saharan Africa. Front Pharmacol. 2020; 11:866. 


\section{Supplementary table 1: Consent form and Interviewer administered questionnaire}

\section{Medicinal plants used among pregnant women admitted in Jimma University Medical Center maternity and gynaecology wards in Jimma city, Ethiopia}

\section{Consent form}

\section{Background and purpose}

This is an invitation for you to participate in a research conducted with the objective of assessing medicinal plants and pharmaceutical medicines used among pregnant women admitted in Jimma University Medical Center (JUMC) maternity and gynecology wards in Jimma city, Ethiopia. Although medicinal plants play a significant role in traditional medicine during pregnancy, childbirth and postpartum care, little is known about the extent and types of medicinal plants used during pregnancy in Ethiopia. The aim of this study is therefore to investigate and describe medicinal plants and pharmaceutical medicines used during pregnancy, the reasons for use and the utilization pattern among inpatient pregnant and lactating women. As the study is directly related to women seeking care in the maternity and gynaecology ward of this hospital, you are one of the candidates who can participate in the study. Thus, you are kindly requested to participate in the present research and provide the information required from you.

\section{What does the study involve?}

Concerning the study process, first we will ask you questions about your background including questions about your age, religion, residence place, occupation, family size, ethnic group, marital status, educational level, access to modern health facility and walking distance to the facility. Next, we will ask you about maternal diseases, pregnancy-related illness and treatments, use of medicinal plants, information about women's safety concerns and experiences with use of medicinal plants in pregnancy. We will further collect data about your chronic diseases and medication history, self-medication with conventional medicines, and social drug use during pregnancy.

\section{Potential advantages and disadvantages}


The results obtained from this study are useful in order to develop better strategies to appropriately use medicinal plants, minimize medicinal plant use related problems and reduce maternal morbidity and mortality. There is not any disadvantage in participating in this study, except the time that it takes to answer the study questions.

\section{What will happen to your personal information?}

The data registered about you will only be used in accordance with the purpose of the study as described above. All the data will be processed without name, personal identification number or other directly recognisable type of information. A code number links you to your data and only the authorized study staff will have access to this list. There will be no way of linking your individual responses to the final result of the study findings. For documentation and follow-up purposes, the data will be kept until 14.01.2024. The data will be stored as deidentified data, i.e. a file with key identifiable information stored separately from the file containing other data. The data will be anonymized within 6 months after this date. It will not be possible to identify you in the results of the study when these are published.

\section{Voluntary participation}

Participation in this study is voluntary. You can withdraw your consent to participate in the study at any time and without stating any particular reason. This will not have any consequences for your further treatment. If you wish to participate, please sign the declaration of consent at the bottom of this page. In case if you are not able to give written consent (i.e. due to literacy and /or cultural reasons), your oral consent will be sought and documented as equal to a written consent. There are no consequences for women who decide not to participate in this study. The patient's decision to participate or not will have no impact on the treatment(s) that she receives.

\section{Right to access and material storage}

If you agree to participate in the study, you are entitled to have access to the information registered about you. You are further entitled to correct any mistakes in the information we have registered. If you withdraw from the study, no further information or material will be collected about you. Data that have already been collected will not be deleted.

\section{Information about the outcome of the study}


You, as a participant in this study, are entitled to receive information about the outcome/result of the study.

\section{Funding}

Mr. Seid Mussa is a PhD student in the University of Oslo. He is a recipient of scholarship from the Norwegian Loan Fund (Lånekassen).

If you have questions concerning the study, you may contact the research team:

\section{Mr. Seid Mussa Ahmed}

Telephone: +251911820125 (Mobile phone), +251471111979 (Office phone)

Email: seid.mussa@ju.edu.et / seidma@studmed.uio.no

School of Pharmacy, Faculty of health sciences, Jimma Institute of Health, Jimma University, Jimma, Ethiopia;

\section{Dr.Yesuf Ahmed Aragaw}

Telephone: +251911004736 (Mobile phone), +251 471110867 (Office phone)

Email: yesuf.aragaw@ju.edu.et / yesufahmed47@yahoo.com

Department of Obstetrics and Gynaecology, Faculty of Medical Sciences, Jimma Institute of Health, Jimma University, Jimma, Ethiopia;

\section{Consent for participation in the study}

I consent to participate in the study.

(Signed by the study participant, date)

Third party consent when this is warranted, either in addition to or in place of the participant's consent.

(Signed by a close relative/partner/friend, date)

I confirm that I have given information about the study.

(Signed by the data collector, date) 


\section{Questionnaire}

Instructions for enumerators:

- Many questions allow multiple answers. Unless specifically instructed in the question, do not prompt and simply encircle the answers that the woman mentions

- For open ended questions please write down the pregnant woman's response legibly

\section{Part I. Socio-demographics characteristics of respondents}

\subsection{Study ID code}

\subsection{What is your age?}

1.3. What is your place of residence?
A. Urban
B. Rural

\subsection{What is your educational level?}
A. Illiterate
B. Read and write but no formal education
C. Primary $1^{\text {st }}$ cycle (1-4)
D. Primary $2^{\text {nd }}$ cycle (5-8)
E. Secondary school (9-12)
F. Post-secondary school
G. Others, specify

\subsection{What is your marital status?}
A. Married
B. Single
C. Divorced
D. Widowed

\subsection{What is your ethnic group?}
A. Oromo
B. Amhara
C. Gurage
D. Dawuro
E. Silte
F. Yem 

G. Tigre
H. Others, specify

1.7. What is your religion?
A. Islam
B. Orthodox Christian
C. Protestant Christian
D. Catholic Christian
E. Others, specify

1.8. What is your occupation?
A. Farmer
B. House wife
C. Trader/Merchant
D. Government employee
E. Private employee
F. Daily labourer
G. Others, specify

1.9. How many family members do you have (including yourself)?

1.10. Do you have access to any modern health facility (especially in 5 to $10 \mathrm{~km}$ walking distance from your residence)? (If no skip to $Q$ 2.1)
A. Yes
B. No

1.11. How many minutes walking distance is it to your nearest health facility?

\section{Part II. Pregnancy-related questions}

2.1.Are you pregnant? (If no skip to $Q$ 2.3)
A.Yes
B. No

2.2. In which week of pregnancy (gestation age) are you?

2.3. How many days have passed since delivery?

2.4. How many children do you have from before the current pregnancy?

2.5. How many times have you been pregnant (i.e. Gravida)?

2.6. The number of times your pregnancies reaching viable gestational age (including live births and stillbirths, i.e. parity)

2.7. History of any adverse pregnancy outcome? (If no skip to $Q 3.1$ ) 

A. Yes
B. No

2.8. What type (s) of adverse pregnancy outcome?
A. Down syndrome
B. Cleft lip/ palate
C. Neural tube defect
D. Cardiac defect
E. More than one/ mixed [please explain]
F. Others, specify

2.9. Have you used iron sulphate during pregnancy? (If no skip to $Q 3.1$ )
A. Yes
B. No

2.10. When did you use?
A. First trimester (first three months of pregnancy)
B. Throughout the entire pregnancy
C. Before and during pregnancy
D. Others, specify

\section{Part III. Chronic disease and medication}

3.1. Do you have chronic disease? (If no skip to $Q$ 4.1)
A. Yes
B. No

3.2. What is the chronic disease?
A. Hypertension
B. Diabetes mellitus
C. Asthma
D. Cardiac diseases
E. Liver disease
F. Chronic renal failure
G. Gastritis/peptic ulcer
H. HIV/AIDS
I. Others, specify

3.3. Do you take drugs for the management of chronic illness? (If no skip to $Q$ 3.5)

A.Yes 
B. No

3.4. What type of drugs are you taking [names of drugs]?

3.5. Are you currently attending chronic disease follow-up clinic?
A. Yes
B. No

Part IV. Self-medication with conventional medicines

4.1. Have you ever practiced self-medication (to treat self-diagnosed disorders or symptoms) with conventional medicines during pregnancy? (If no skip to Q 5.1)
A. Yes
B. No

4.2. Which drugs did you use for self-medication?
A. NSAIDs (write drug name (s))
B. Dermatologicals (write drug name (s))
C. Antimicrobials (write drug name (s))
D. Others, Specify

4.4. Had you received any advice /counselling on self-medications drugs? (If no skip to Q 5.1)
A. Yes
B. No

4.5. For which of the following points you had received advice?
A. Tolerable side effects of drugs
B. Adverse drug reactions which requires prescribers visit
C. Management of missed dose
D. How to take the medication
E. Others specify

\section{Part V. Social drug use during pregnancy}

5.1. Do you smoke cigarette? (If no skip to Q 5.2.)
A. Yes
B. No

5.2. How many cigarettes do you smoke per day?

5.3. For how many years have you smoked?

5.4. Do you drink alcohol? (If no skip to Q 5.3.) 

A. Yes
B. No

5.5. Which type of alcohol do you drink?
A. Tella (Local beer)
B. Katikala ('Ethiopian vodka')
C. Beer
D. Wine
E. Others, specify

5.6. What millilitre per day do you drink?

5.7. For how many years have you drunk?

5.8. Do you chew Khat? (If no skip to Q 6.1.)
A. Yes
B. No

5.9. What is the average weight in "zurba" that you chew daily?

5.10. For how many years have you chewed?

5.11. Any other social drug you used?

Part VI. General questions about medicinal plants used during pregnancy

6.1. Have you used any medicinal plants to manage your current pregnancy illness? (If yes skip to Q. 6.4; if no skip to Q. 6.2 and Q. 6.3, and after that thank the woman and stop the interview)
A. Yes
B. No

6.2. Why didn't you use medicinal plants in pregnancy?
A. Fear of complications to the baby
B. Religious belief
C. Not aware of their use in pregnancy
D. Counseled by the health worker
E. Others, specify

6.3. Outcomes of previous pregnancy for non-users of medicinal plants?
A. Alive
B. Neonatal death
C. Stillbirth 
D. Abortion

E. Others, specify 


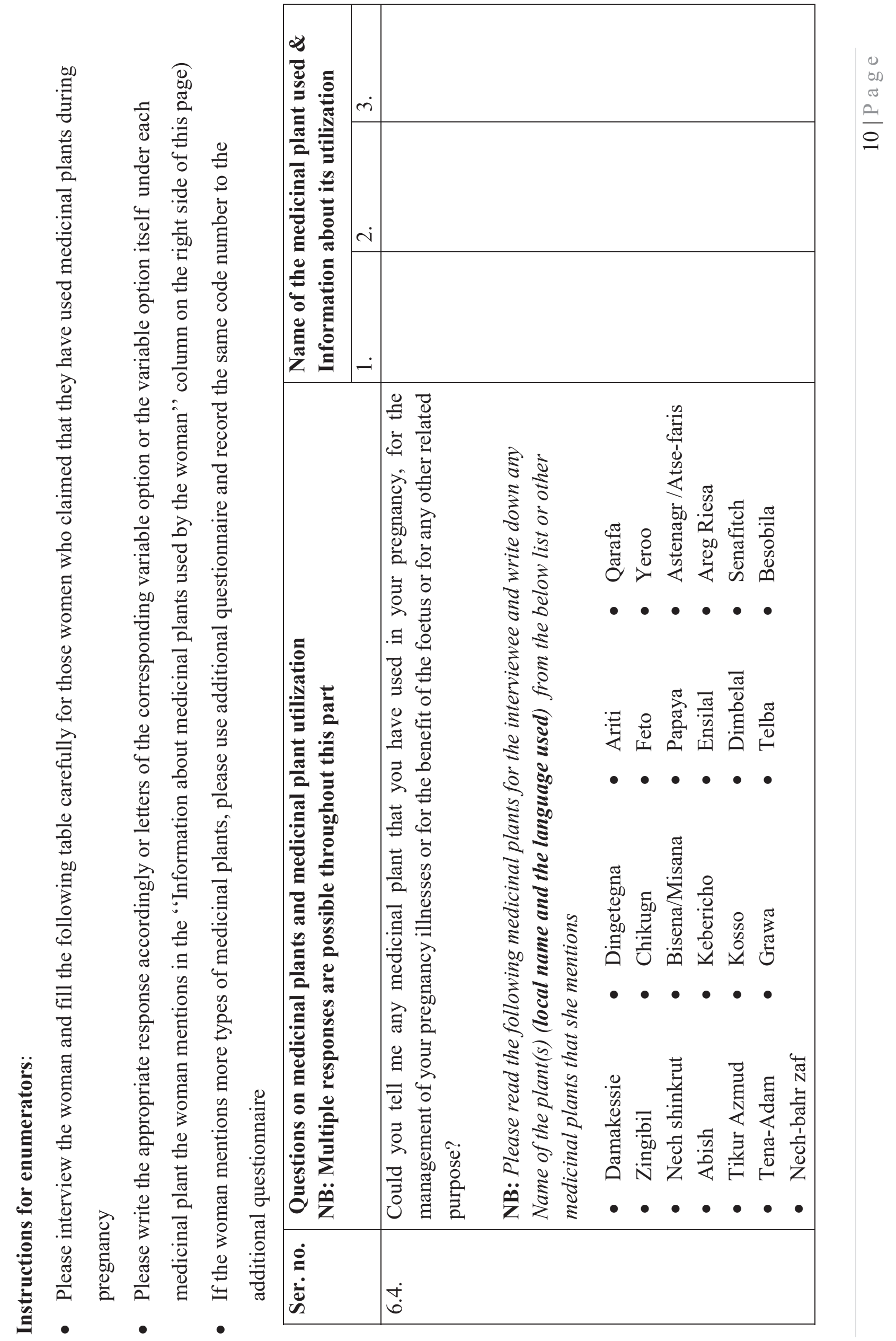




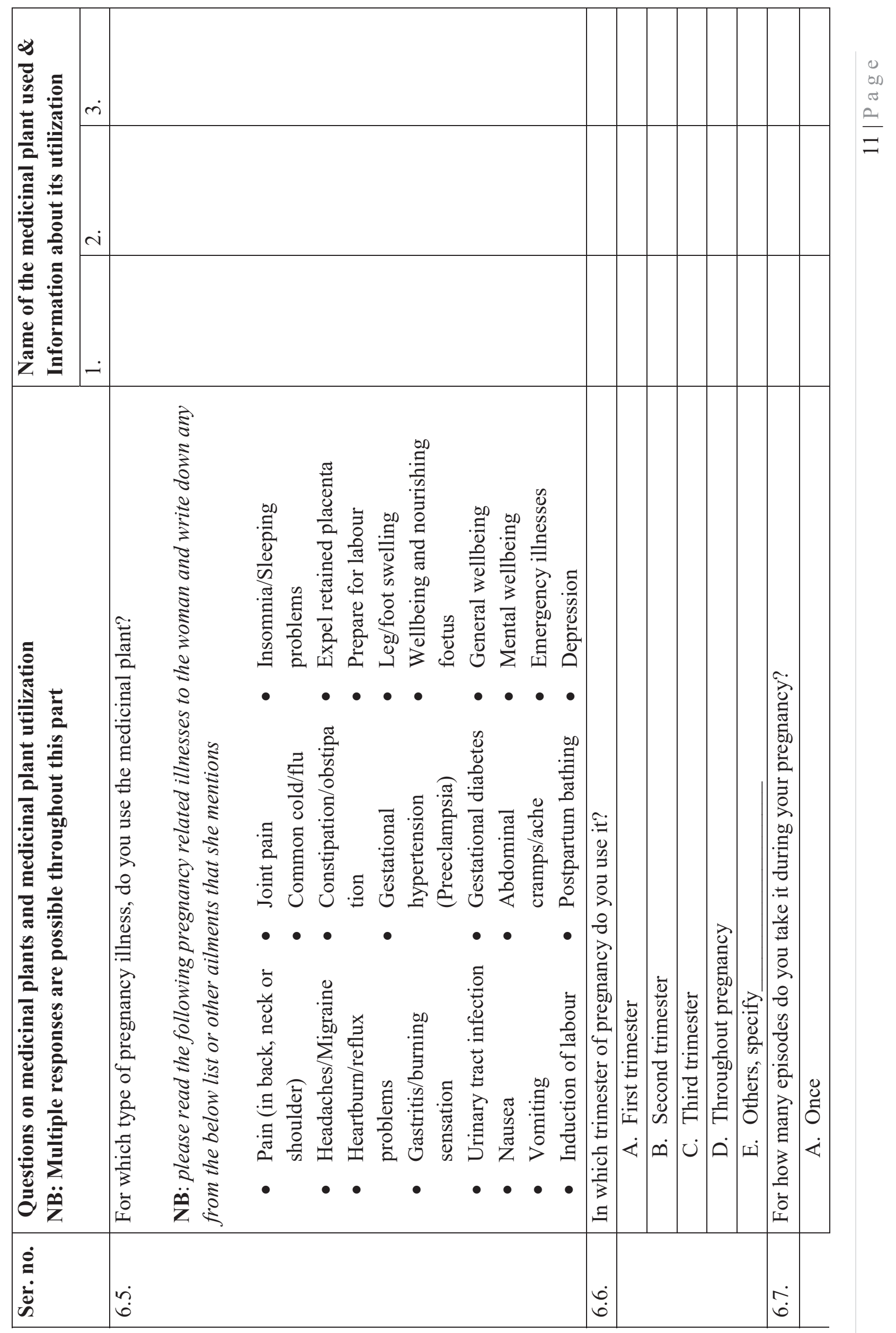



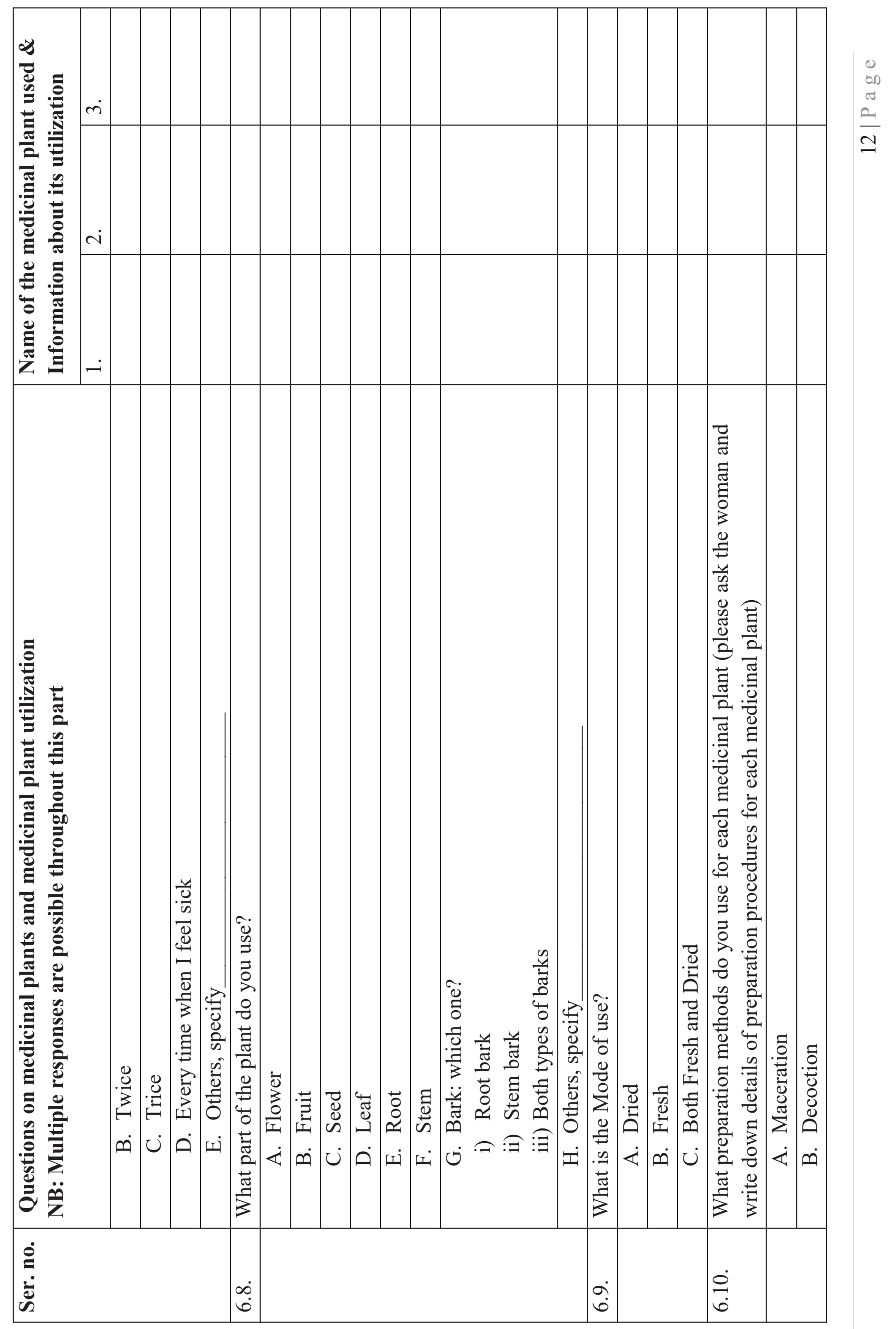

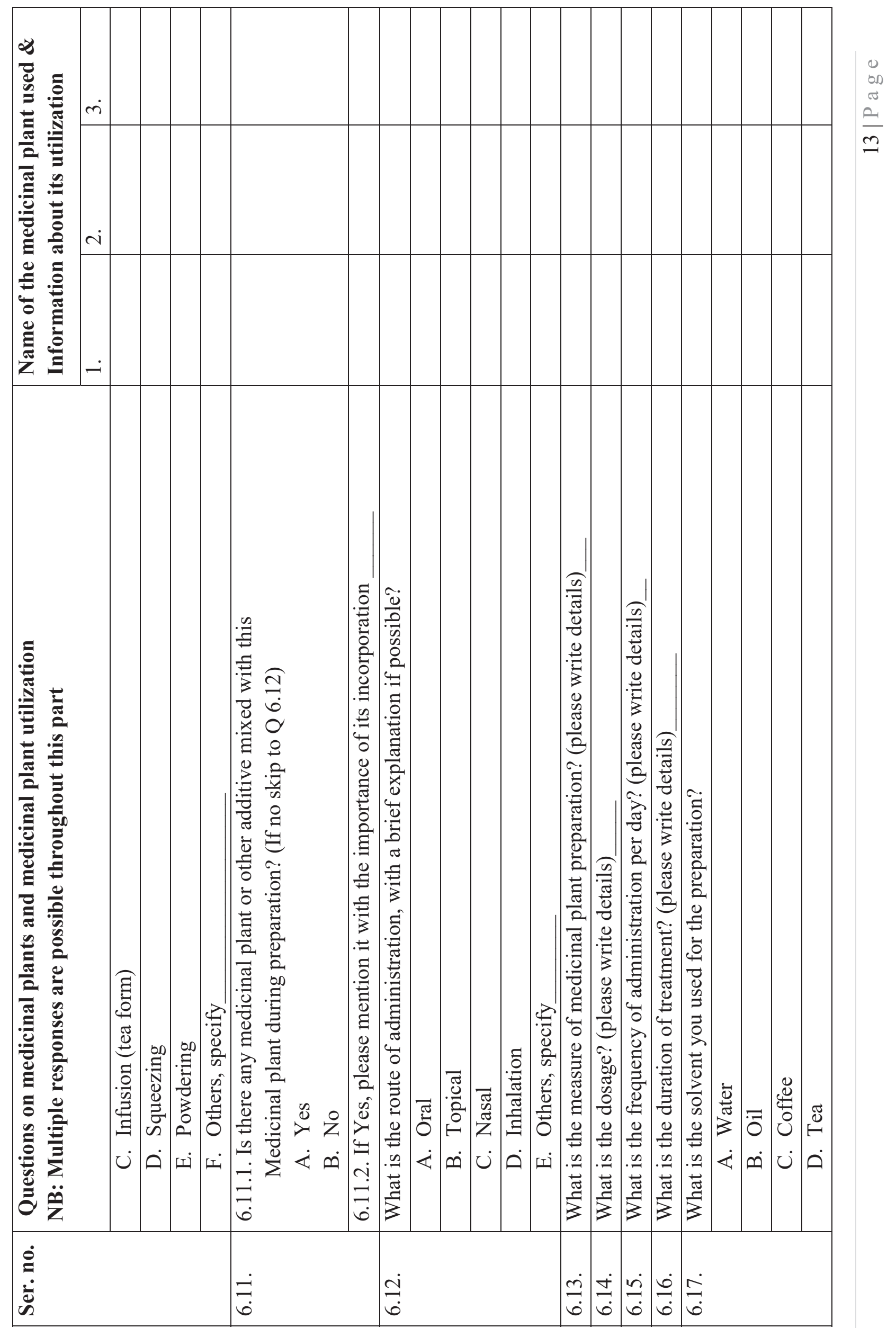


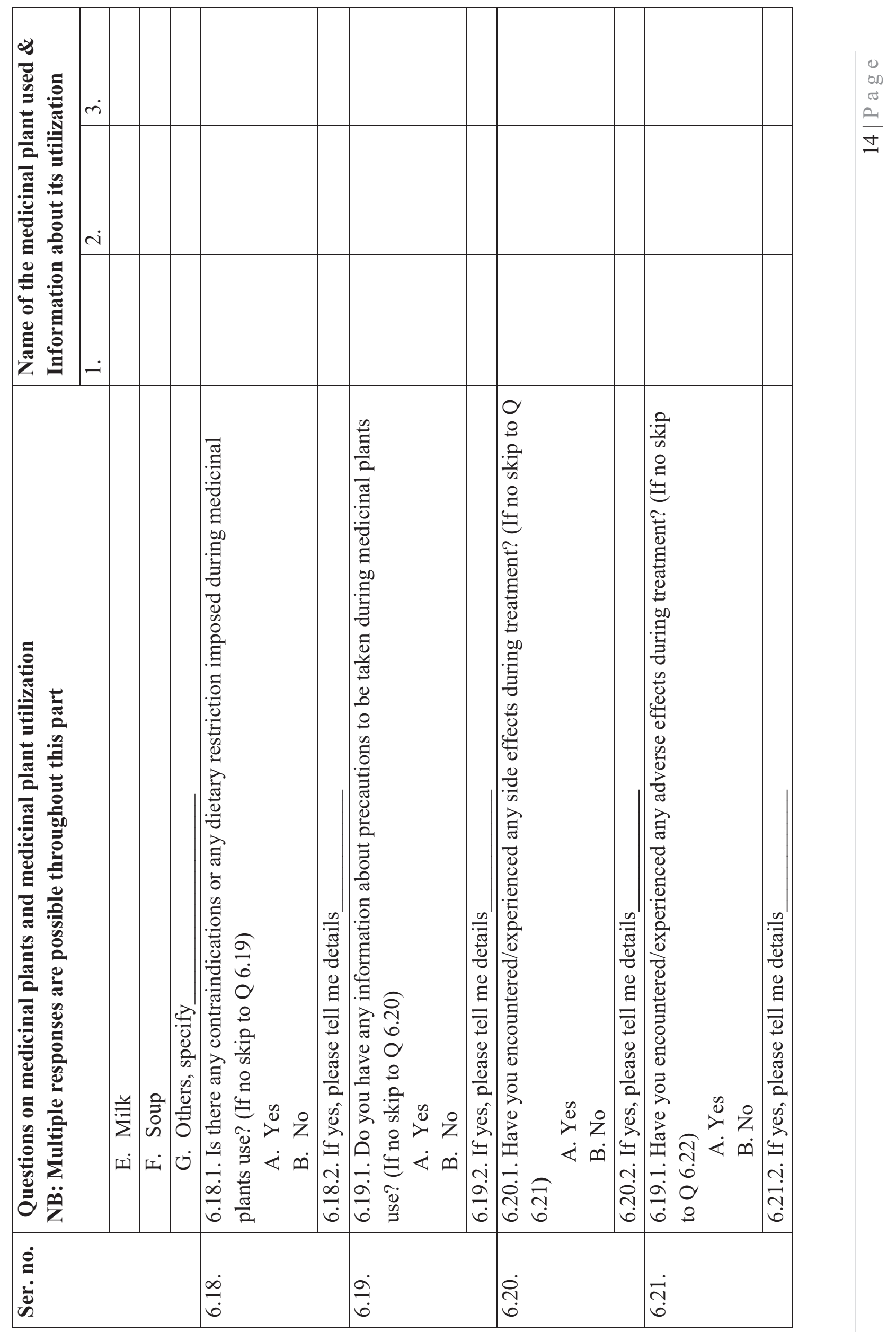




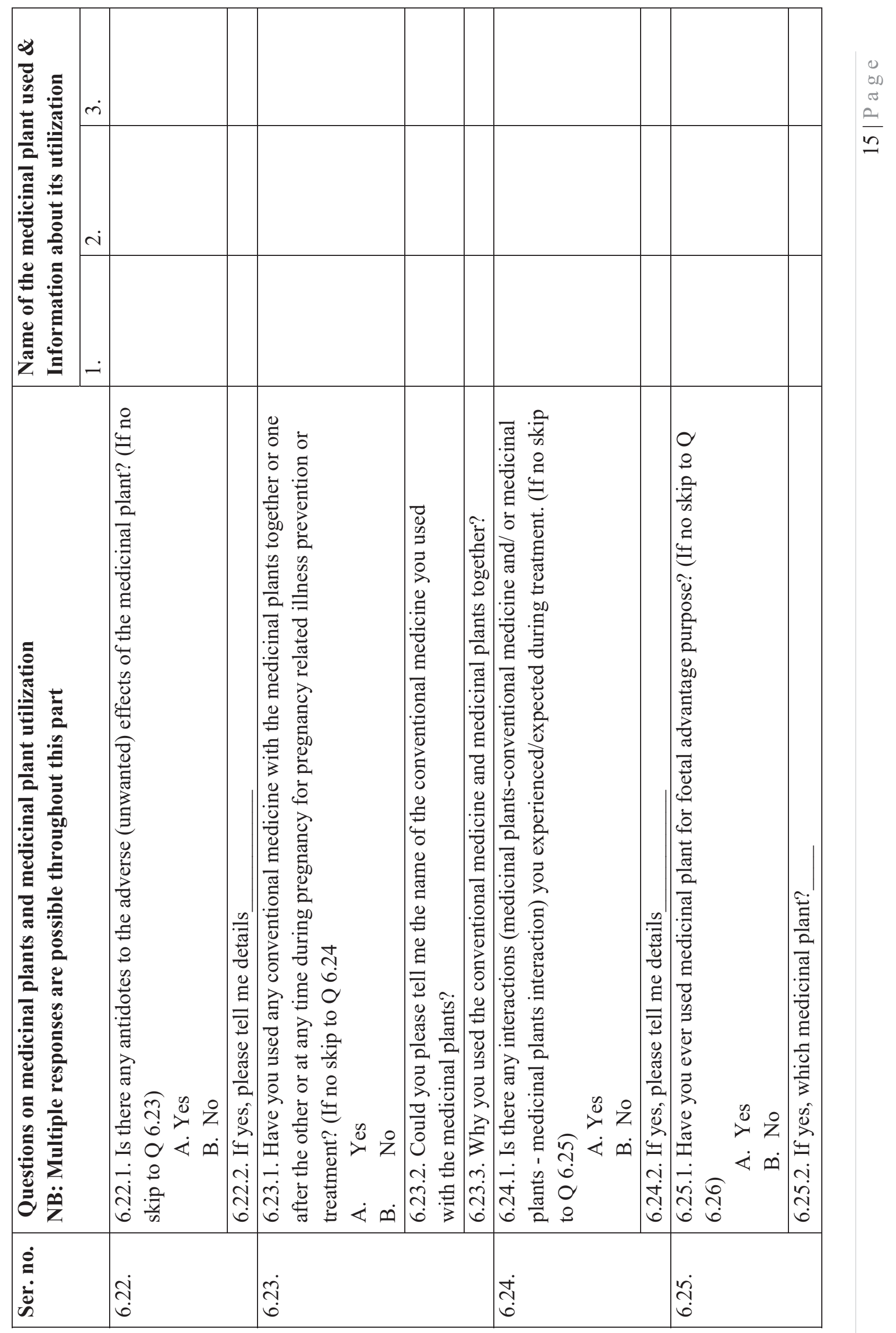




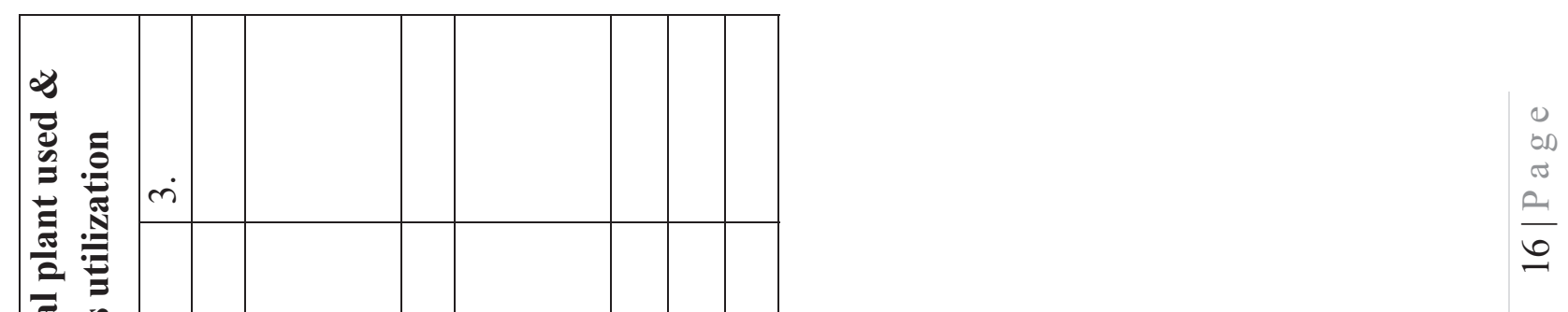




\section{Part VII. Sources of information and medicinal plants used during pregnancy}

7.1. What is your source of medicinal plants?
A. Market places
B. Traditional healers (herbalist)
C. Garden
D. Shop
E. Neighbor
F. Others, specify

7.2. Who helps you in the collection of the medicinal plants?
A. Family members (mother, father, husband, grandmother, etc.)
B. Neighbours
C. Friends
D. My-self
E. Others, specify

7.3. Who recommended you to use medicinal plants during pregnancy?
A. Family members (mother, father, husband, grandmother, etc.)
B. Neighbours
C. Friends
D. My-self
E. Others, specify

7.4. If anyone recommended you, did you get any information how to use medicinal plants?
A. Yes
B. No
C. Others, specify

7.5. Were you satisfied with medicinal plant treatment outcomes? (If yes, finish!)
A. Yes
B. No

7.6. Why you were not satisfied?
A. Got abortion
B. Uterine hyper-stimulation
C. Fetal distress
D. Stillbirth
E. Uterine rupture
F. Any other reason, specify

7.7. Will you use medicinal plants in your future pregnancy?
A. Yes
B. No

I thank you for your time and cooperation! date 


\section{Supplementary table 2: Data extraction form for patient medical record review}

Basic admission details and patient characteristics:

- Study Id ; Admission ward: Maternity/labour

Gynaecology

- Age $\square$; Weight ; Height

- Admission date Discharge date Duration of hospitalization

- Gestational age (in weeks)

- Gravidity: ; Parity

- Type of patient: Antenatal Postnatal

- Type of delivery: Vaginal delivery

$\square$ Cesarean delivery

- Breast feeding :Yes No

- Known drug allergies: Yes No $\square$; Type of drug allergy Details of admission (including vital signs):

Pregnancy outcomes and other obstetrics data (live birth, stillbirth, twin birth, postpartum haemorrhage, congenital abnormalities/birth defects, hypertension/eclampsia/HELLP, diabetes, placental abruption, etc.):

Any other maternal and perinatal outcomes:

Relevant laboratory results and investigations (Renal function test, Liver function test, Complete Blood Count (CBC), Echocardiography, Lipid Profile, Cardiac function, Electrolyte test, Glycaemic level, etc.): 
Supplementary table 3: Definitions of safety categories of medicinal plants used during pregnancy at JUMC, Ethiopia

Classification

Description

Safe to use in pregnancy Available human evidence suggests the medicinal plant can be safely used in pregnancy

Use with Caution Available human evidence for the medicinal plant is limited so it should not be used without consulting a qualified health care practitioner

Potentially harmful to use Available evidence has shown adverse impacts on in pregnancy pregnancy or fetus following the use of the medicinal plant

Information unavailable No reference was found regarding use of the medicinal plant in pregnancy

Source [adapted from]: Kennedy DA, Lupattelli A, Koren G, Nordeng H. Safety classification of herbal medicines used in pregnancy in a multinational study. BMC Complement Altern Med. 2016; 16: 102. 
Supplementary table 4: Overview of medicinal plants used during pregnancy according to safety classification and number of users at JUMC, Ethiopia

\begin{tabular}{|c|c|c|c|}
\hline Type of medicinal plant used & $\begin{array}{l}\text { Safety } \\
\text { class* }\end{array}$ & $\begin{array}{l}\text { Number } \\
\text { of users } \\
(\mathrm{N}=319)\end{array}$ & $\begin{array}{l}\text { Percen } \\
\text { tage }^{\#}\end{array}$ \\
\hline Linum usitatissimum L. (Telba) & Caution & 246 & 77.1 \\
\hline Ocimum lamiifolium L. (Damakessie) & Unavailable & 40 & 12.5 \\
\hline Carica papaya L. (Papaya) & Caution & 35 & 11.0 \\
\hline Zingiber officinale Roscoe. (Zingibil) & Safe & 29 & 9.1 \\
\hline Allium sativum L. (Nech shinkrut) & Safe & 28 & 8.8 \\
\hline Trigonella foenum-graecum L. (Abish) & Harmful & 24 & 7.5 \\
\hline Nigella sativa L. (Tikur Azmud) & Unavailable & 21 & 6.4 \\
\hline Ruta chalepensis L. (Tenadam) & Harmful & 15 & 4.7 \\
\hline Eucalyptus globulus Labill. (Nech-bier zaf) & Safe & 13 & 4.1 \\
\hline Cinnamomum verum J.Presl (Qarafa) & Harmful & 4 & 1.3 \\
\hline Taverniera abyssinica A. Rich. (Dingetegna) & Unavailable & 3 & 0.9 \\
\hline Artemisia abyssinica Sch.Bip. ex A.Rich. (Chikugn) & Harmful & 3 & 0.9 \\
\hline Croton macrostachyus Hochst. (Bisena/Misana) & Harmful & 3 & 0.9 \\
\hline Echinops kebericho Mesfin (Kebericho) & Harmful & 3 & 0.9 \\
\hline Hagenia abyssinica (Bruce ex Steud.) J.F.Gmel. (Kosso) & Harmful & 2 & 0.6 \\
\hline Vernonia amygdalina Del. (Grawa) & Unavailable & 2 & 0.6 \\
\hline Saccharum officinarum L. (Sugar cane) & Safe & 2 & 0.6 \\
\hline Brassica nigra (L.) K.Koch (Senafitch) & Unavailable & 1 & 0.3 \\
\hline Zehneria scabra Sond. (Areg Riesa) & Unavailable & 1 & 0.3 \\
\hline Artemisia afra Jacq. ex Willd. (Ariti) & Harmful & 1 & 0.3 \\
\hline Lepidium sativum L. (feto) & Unavailable & 1 & 0.3 \\
\hline Guizotia abyssinica (L.f.) Cass. (Nug) & Unavailable & 1 & 0.3 \\
\hline Vicia faba L. (faba Beans) & Unavailable & 1 & 0.3 \\
\hline Ananas comosus (L.) Merr. (Annanas) & Caution & 1 & 0.3 \\
\hline Phoenix dactylifera L. (Temir) & Safe & 1 & 0.3 \\
\hline Pycnostachys abyssinica Fresen. (Yeroo) & Unavailable & 1 & 0.3 \\
\hline Bahuu/B'auu (Oromiffa language name) & Unavailable & 1 & 0.3 \\
\hline
\end{tabular}

*Safe: safe to use in pregnancy; Caution: requires cautious to use in pregnancy; Unavailable; information on safety to use in pregnancy was not available in the current literature;

Harmful: potentially harmful to use in pregnancy [Contraindicated]; ${ }^{\#}$ Total percentage may exceed $100 \%$ due to multiple responses 


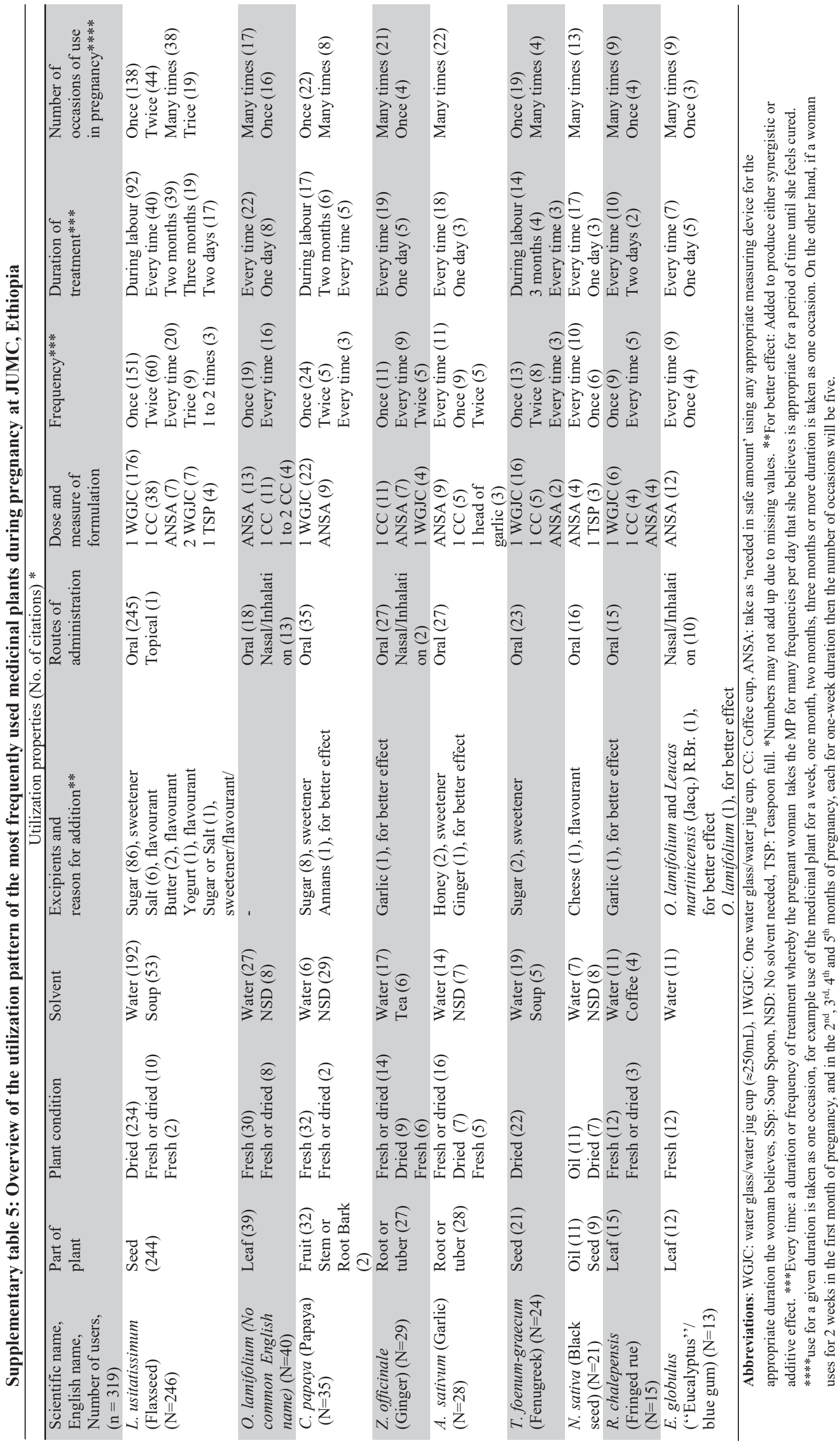



III 



\title{
Self-Medication and Safety Profile of Medicines Used among Pregnant Women in a Tertiary Teaching Hospital in Jimma, Ethiopia: A Cross-Sectional Study
}

\author{
Seid Mussa Ahmed ${ }^{1,2, * \mathbb{D}}$, Johanne Sundby ${ }^{1}$, Yesuf Ahmed Aragaw ${ }^{3}(\mathbb{D})$ and Fekadu Abebe ${ }^{1}$ \\ 1 Department of Community Medicine and Global Health, Institute of Health and Society, Faculty of Medicine, \\ University of Oslo, P.O. Box 1130 Blindern, N-0318 Oslo, Norway; johanne.sundby@medisin.uio.no (J.S.); \\ fekadu.abebe@medisin.uio.no (F.A.) \\ 2 Division of Social and Administrative Pharmacy, School of Pharmacy, Faculty of Health Sciences, Jimma \\ Institute of Health, Jimma University, Jimma P.O. Box 378, Ethiopia \\ 3 Department of Obstetrics and Gynaecology, Faculty of Medical Sciences, Jimma Institute of Health, Jimma \\ University, Jimma P.O. Box 378, Ethiopia; yesufahmed47@yahoo.com \\ * Correspondence: seidma@studmed.uio.no
}

Received: 2 May 2020; Accepted: 27 May 2020; Published: 4 June 2020

\begin{abstract}
Background: Despite the potential foetal and maternal risks of self-medication, studies on self-medication and safety profile of medicines used during pregnancy are scarce. This study determined the prevalence, predictors and safety profile of medicines used for self-medication during pregnancy at Jimma University Medical Centre (JUMC) in Ethiopia. Methods: A hospital-based cross sectional study was conducted on 1117 hospitalized pregnant women or postpartum women in the maternity and gynaecology wards at JUMC between February and June 2017. Data were collected using an interviewer-administered structured questionnaire and by reviewing patient medical records. Data were analysed using descriptive statistics and logistic regression. Result: Nearly 3 out of 10 women reported taking at least one type of conventional medicine for self-medication, mainly analgesics $92.3 \%$. Almost $75.0 \%$ of the self-medicated women used medicines classified as probably safe and $13.6 \%$ as potentially risky to use during pregnancy. Medicinal plant use, religion and access to a health facility near their residency were significantly associated with self-medication during pregnancy. Conclusions: Self-medication is common among pregnant women at JUMC. Most women used medicines classified as safe to use during pregnancy. There is need for enlightenment of pregnant women on the potential dangers of self-medication during pregnancy to prevent foetal and maternal risks.
\end{abstract}

Keywords: pregnancy; self-medication; conventional medicine; safety; Ethiopia

\section{Introduction}

Pregnancy is a dynamic process in which many maternal physical, physiological, pharmacokinetic and pharmacodynamic transformations occur from fertilization to parturition [1-3]. Changing hormones can alter a woman's mood and cause nausea, vomiting, heartburn, constipation, headache, cough and other weakening pregnancy pains that force expectant mothers to seek healthcare [2-5]. However, in most developing countries like Ethiopia not only are health care facilities inaccessible or unaffordable $[2,4,6]$, but also medicines are poorly regulated and easily available outside formal and authorized institutions [7]. For this reason, many pregnant women prefer to self-medicate first as an accessible and lower cost alternative and only seek professional health services when the situation worsens $[4,8]$. 
Self-medication is defined as the use of medicines or the intermittent or continued use of prescribed medicines by the patient to treat self-diagnosed disorders or symptoms on their own initiative [8,9]. What makes self-medication more dangerous in developing countries is that the population has low health literacy, and thus may have a poorer understanding of health information and inappropriate use of medicines $[8,10]$. In Ethiopia, the prevalence of self-medication with conventional medicines in pregnancy has been shown to vary from $1.9 \%$ [11] to $29.1 \%$ [12]. Patients self-medicating with western medicine may use medicinal plants concomitantly and often without the knowledge of a healthcare professional, which might further threaten the pregnancy [2,13]. Furthermore, medicines taken by the mother may cause serious structural and functional adverse effects on the developing baby including developmental delay, foetal toxicity, low birth weight, intellectual disability, birth defects, miscarriage and stillbirth $[7,14]$.

Self-medication in pregnancy is common in Sub-Saharan African countries, potentially exposing the unborn child to medicines that may have risk [15]. Potentially risky medicine use in pregnancy has been reported, with prevalence estimates of 28.0\% in Europe [16] and 49.5\% in Burkina Faso [15].

Different classification systems of risks of medicines used during pregnancy have been established depending on foetal safety. The most well-known are the U.S., the Australian and the Swedish pregnancy risk classification systems [17]. Despite their limitations, these systems are important in describing medicine utilization patterns [16].

In addition to self-medication, pregnant women may be unaware that social drug use could affect the health of the foetus. Substance use during pregnancy has been associated with miscarriage, stillbirth, preterm birth, restricted foetal growth, low birth weight, babies who are small for their gestational age and intellectual impairment in children and can contribute to maternal or delivery complications [3,18,19]. Smoking as high as 5.0\% [20], alcohol consumption as high as 39.8\% [18], and khat (Catha edulis) chewing varying from 10.0\% [21] to 35.8\% [22] has been reported in pregnant women in Ethiopia.

The government of Ethiopia is working hard to achieve Sustainable Development Goal 3 (SDG 3) to reduce the maternal mortality ratio (MMR) from 412 deaths per 100,000 live births in 2015 to less than 70 deaths per 100,000 live births by 2030 [23]. Identifying the extent and determinants of self-medication, safety profile of medicines used for self-medication and social drugs used could help in educating and counselling pregnant women and in achieving the SDG 3 targets. However, in Ethiopia, despite the fact that its negative pregnancy outcomes pose a significant threat to maternal and foetal health [24], data systematically examining prevalence and correlates of self-medication in pregnancy are still scarce $[7,12,25,26]$. Moreover, the limited self-medication studies performed in the country have been confined to outpatient pregnant women $[7,12]$. Since we did not find any study that assessed self-medication experience and safety profiles of medicines used among hospitalized pregnant women, this study aimed to determine the magnitude of self-medication practice and its determinants among pregnant women at JUMC. The study also evaluated pregnancy safety profiles of medicines used based on risk classification techniques developed in the U.S. and Australia. A secondary aim was to assess the prevalence and types of social drugs used among the pregnant women at JUMC.

\section{Subjects and Methods}

\subsection{Study Design and Setting}

A facility based cross-sectional study was conducted in the maternity and gynaecology wards of a tertiary care teaching hospital, JUMC, Ethiopia. Geographically, the hospital is located in Jimma city $350 \mathrm{~km}$ southwest of the capital city of Ethiopia, Addis Ababa. It is one of the oldest public hospitals in the country established in 1937/38 [27]. Currently it is the only teaching and referral hospital in the south-western part of the country, with a catchment population of about 20 million people [28]. The JUMC obstetrics and gynaecology department has a bed capacity of 265 and provides specialized health services for about 7600 inpatients and 11,600 outpatients every year. 
The department of obstetrics and gynaecology has two wards (maternity and gynaecology), one antenatal care (ANC) outpatient clinic, one general gynaecological outpatient clinic and one family planning clinic [28]. Women with a gestational length of 28 weeks or higher and women in labour receive care in the maternity ward. Women with less than 28 weeks of pregnancy (most often hyperemesis and abortions) are treated at the gynaecology ward. The gynaecology ward also manages and treats gynaecological conditions in non-pregnant patients. Ethics approval and consent to participate: The study was approved by the Regional Committees for Medical and Health Research Ethics (REC) in Norway (Ref.: 2015/2135, REK Sør-Øst B), dated 17 December 2015 and the Institutional Review Board (IRB), Institute of Health, Jimma University, Ethiopia (ref. no IHRPGC 7206/07), dated 17 January 2017. Permission was secured from JUMC before commencing the study. Written informed consent was obtained from each study participant before data collection. All information obtained from participants during the study was kept confidential.

\subsection{Study Population and Sample Size}

Pregnant or postpartum women in the maternity and gynaecology wards at JUMC were invited to participate in the study during their inpatient hospital stay. Since there are no previous studies that reported the prevalence of self-medication with conventional medicine among pregnant women prior to hospital admission, we used $50 \%$ as a conservation estimate. The sample size was based on having a power of $80 \%$, a critical level of significance of $5 \%$ and an error margin of $3 \%$ using the Kish single population formula [29] provided below in Equation (1). Sample size calculation was based on the study's primary objective, that is, to provide an estimate of self-care with conventional medicines among pregnant women prior to hospitalization.

$$
N=Z_{\alpha / 2}^{2} \times \frac{p(1-p)}{\varepsilon^{2}}=1.96^{2} \times \frac{0.5(1-0.5)}{0.03^{2}}=1067
$$

$N$ is the sample size; $Z$ is the standard normal deviate (the $Z$ value for $95 \%$ confidence level is 1.96 ).

We also allowed the possibility of a $5 \%$ non-response rate (approximately 54 women). Therefore, at least 1121 women were required for the study to have enough power.

\subsection{Inclusion and Exclusion Criteria}

Inclusion criteria: Pregnant or postpartum patients aged $\geq 18$ years admitted in the maternity/labour and gynaecology wards at the time of data collection and willing to participate in the study.

Exclusion criteria: Women whose physical and psychological health limited them from providing information, such as those who were unable to speak or mentally disabled, too ill to participate or hard of hearing, were excluded from the study. In addition, women who were unwilling to participate, admitted for less than four hours, under 18 years of age and non-pregnant women admitted in the gynaecology ward were excluded.

\subsection{Data Collection}

Data were collected using a pre-tested face-to-face interviewer-administered structured questionnaire and by reviewing patient medical records. A patient chart review was used to collect pregnancy characteristics, pregnancy outcomes and other medical information about pregnant women. Before the interviews, the aims, objectives and procedures of the study were clearly explained to the participants. After securing written informed consent from each hospitalized pregnant or post-partum woman, the women were consecutively interviewed from February to June 2017. In addition, data were collected at an appropriate and convenient time for the women. Nine trained data collectors, five clinical pharmacists and four nurses from JUMC collected the data. They were given training on how to interview patients using the questionnaire and verify the completeness of the filled questionnaire and abstract information from patient medical records. One of the investigators supervised the data 
collection and verified the completeness of each questionnaire every day. To ensure confidentiality, the questionnaire did not include the woman's name or any other identifying information.

\subsection{Development of Data Collection Tool}

The bilingual questionnaire was developed based on a review of relevant literatures. It was initially developed in English, then translated into the local languages, Amharic and Afan Oromo, and back into English to ensure consistency. The data collection tool was pre-tested on a sample of 30 inpatient pregnant or post-partum women at Shenen Ghibe district hospital located in Jimma city, to assess content validity, content consistency, comprehension and possible defective questions and the time needed to complete it. Based on the pre-test, the questionnaire was amended accordingly and data collectors were clarified on items which were not understood well. The data extraction form was single page and required only minor amendments.

The questionnaire was comprised of four sections to address the aims of this study. Section 1 contained questions about the women's socio-demographic characteristics including age, religion, place of residence, occupation, family size, ethnic group, marital status, educational level and access to a modern health facility. Section 2 contained questions about history of maternal medical problems and maternal and perinatal outcomes. Pregnant women were asked specifically about medical history, pregnancy illnesses and known chronic diseases. Section 3 contained questions about self-medication practice. Self-medication practice was assessed by asking women to list any medication they used by themselves including medications leftover from previous facility visits, bought without a prescription paper from drug retail outlets or shared by anyone and used for the management of their illnesses. Participants were also asked to provide names for any supplement or preparation they may have taken including iron, folic acid and any other supplement. Section 4 covered social drug use history, particularly tobacco smoking, alcohol drinking and khat chewing during pregnancy. To investigate the use of social drugs, women were asked if they had used any of the listed social drugs-tobacco, alcohol and khat. Moreover, participants were asked to indicate any other social drug used, the amount and the duration of use.

In addition to the questionnaire, a data extraction form was used to collect information about pregnancy characteristics, pregnancy outcomes and other obstetrics information including parity, gravidity, gestational age, delivery route and length of hospital stay. Moreover, maternal and perinatal outcomes of the current pregnancy were collected. Data were extracted by reviewing patients' medical cards.

In this study, concomitant use of medicinal plants and pharmaceutical medicines was assessed by identifying those women who used both during pregnancy for the same or different illnesses.

\subsection{Safety Classification of Medicines}

To attribute each medicine in risk groups according to foetal safety, medicines were classified using two globally recognised risk classification systems commonly used in Ethiopia, the U.S. Food and Drug Administration (US FDA) and the Australia Therapeutic Goods Administration (AU-TGA). The FDA classification system, which uses five categories, A, B, C, D and X [30] was used as the primary categorization approach because it is widely used in Ethiopia. The FDA Category A indicates the safest medications, whereas category $X$ designates medications that have been shown to be teratogenic. The FDA amended their pregnancy risk letter categories in June 2015 and this type of categorization is no longer used [30]. However, it was not only in use during this study but also still widely used in Ethiopia [7,11]. If a particular medicine was not covered by the FDA classification, the AU-TGA classification system [31] was used as a secondary method of classification. It has classes (A, B1, B2, B3, C, D and X) to define medicine safety. Based on similar previous studies $[15,16]$, in order to facilitate the safety analysis and to make categories of more clinical interest, medicine exposures were classified into "probably safe", "potentially risky" or "unclassified". For pharmaceuticals manufactured with several active ingredients; the risk classification was done based on the active ingredient with the highest risk. 
Similarly, for combination medicines, risk class was assigned based on the dominant active substance. According to these two classifications, the "probably safe" medicines group consisted of the FDA categories A and B, and the AU-TGA categories A, B1 and B2. Categories C, D and X for FDA and categories B3, C, D and X for AU-TGA are classified as "potentially risky". Medicines that could not be classified by any of these resources were registered as "unclassified". Finally, when necessary, findings from the FDA or AU-TGA groupings were modified guided by the Ethiopian epidemiological profile, national formulary and treatment guidelines for disease treatment and the WHO recommendations.

\subsection{Statistical Analysis}

One of the investigators (SMA) verified the filled-in questionnaire for completeness and consistency and then coded, entered, cleaned and finally analysed using Statistical Package for the Social Sciences (SPSS) software version 25.0 for Windows (IBM ${ }^{\circledR}$ SPSS ${ }^{\circledR}$ Statistics for Windows, Version 25.0, IBM Corp, Armonk, NY, USA). Descriptive statistics were used to summarize the data at baseline. To identify independent factors significantly associated with self-medication, univariate and multivariate logistic regression analyses were computed and expressed as crude and adjusted odds ratios (ORs) with 95\% confidence intervals (CIs). Independent variables with $p<0.25$ in a univariate logistic regression model were fit into a multivariate model to determine predictors of self-medication. Significance was set at the standard alpha of 0.05 . Whenever the $p$-value was found to be $<0.05$, the association was considered statistically significant. Similar data processing and analysis procedures were used for social drugs.

\section{Results}

\subsection{Study Population Characteristics}

Out of the 1137 pregnant and nursing women invited to participate in the study, 1121 of them agreed to take part, making the response rate $98.6 \%$. However, responses from four women were incomplete and thus complete data on 1117 women (18-45 years old, with a median age 25 years) were analysed. Most of the study participants were urban inhabitants (53.3\%), housewives (46.9\%) and had attended primary school or were able to read and write (42.3\%). Around two-thirds were from Oromo ethnic group (69.7\%), professed the religion of Islam (65.4\%), had a household size less than five $(65.4 \%)$ and lived in a place within walking distance of less than half an hour from the nearest health facility $(66.4 \%)$. The majority were married women $(96.3 \%)$ and admitted in the maternity ward $(88.8 \%)$ and over half of them (54.7\%) delivered vaginally. Five percent of the participants had known chronic disease. The frequencies and percentages of sociodemographic and pregnancy related characteristics of participants are presented in Table 1.

\subsection{Self-Medication Practice}

Table 2 depicts the medicines used for self-medication, the Anatomical Therapeutic Chemical Classification System (ATC) code and the medicine safety category. Of all the pregnant women surveyed, (27.0\%) reported taking at least one type of conventional medicine for self-medication, mainly analgesics (92.3\%) followed by antibiotics (6.7\%) and gastrointestinal (GI) medicines (4.3\%). With regard to specific medicines, paracetamol (72.7\%) followed by diclofenac $(11.0 \%)$ and amoxicillin $(5.7 \%)$ were the most commonly used medicines for self-medication. Nearly a third of women (28.6\%) had used one or more medicinal plant during their current pregnancy. Moreover, 110 (36.7\%) of the self-medicated women had also used one or more medicinal plants along with the conventional medicines during pregnancy. The women used 1 to 8 types of medicinal plants, with an average of 1.63 plants per woman. The majority of these women, 67 (60.9\%), used one medicinal plant, whereas $27(24.5 \%)$ used two and $12(10.9 \%)$ used three. Moreover, two women used four, one woman used six and another woman used eight medicinal plants along with self-medication during pregnancy. Telba (Flaxseed) $88(80.0 \%)$ was the most commonly used medicinal plant followed by Zingibil (Ginger) 15 $(13.6 \%)$ (Table S1). 


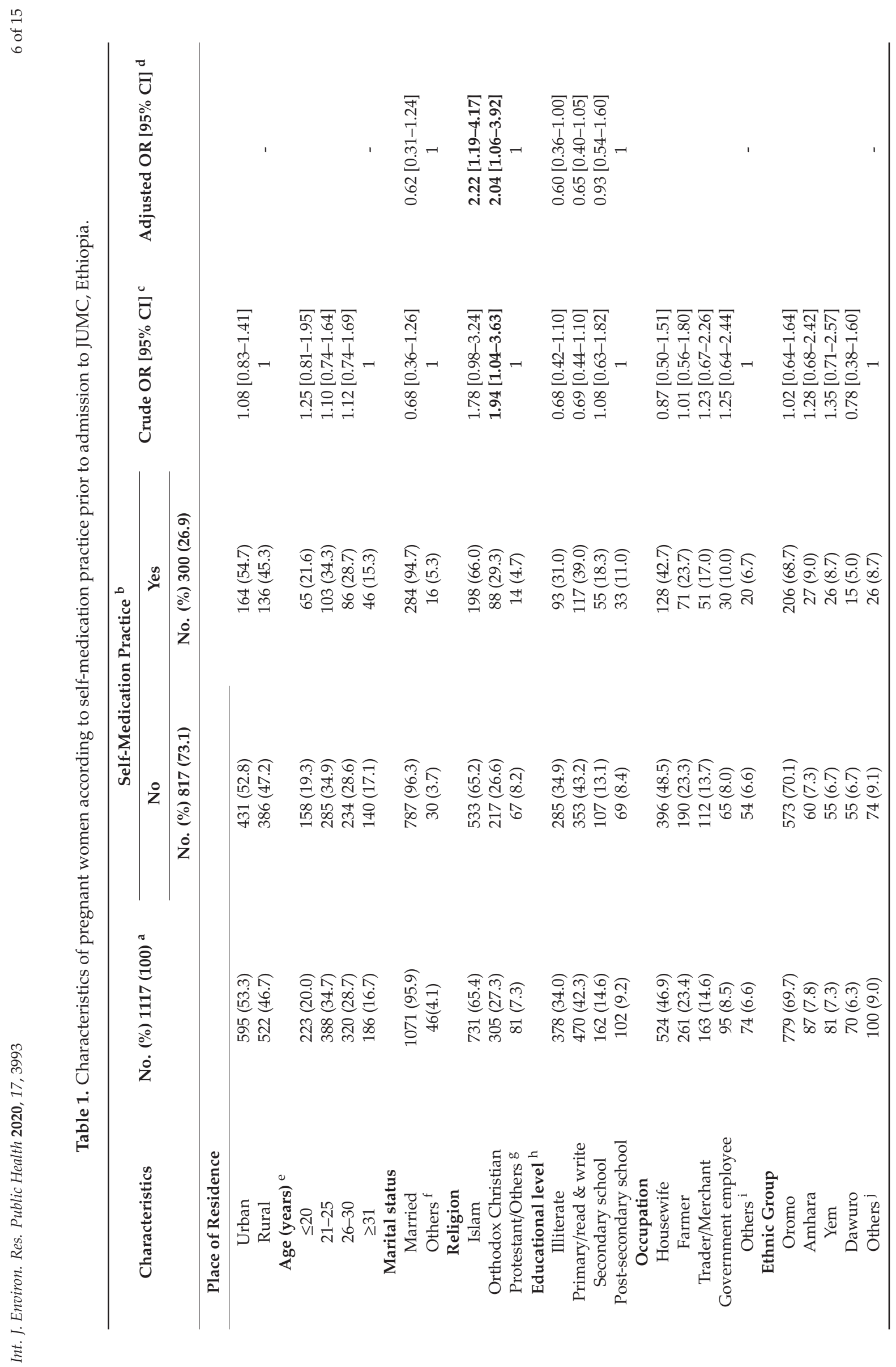




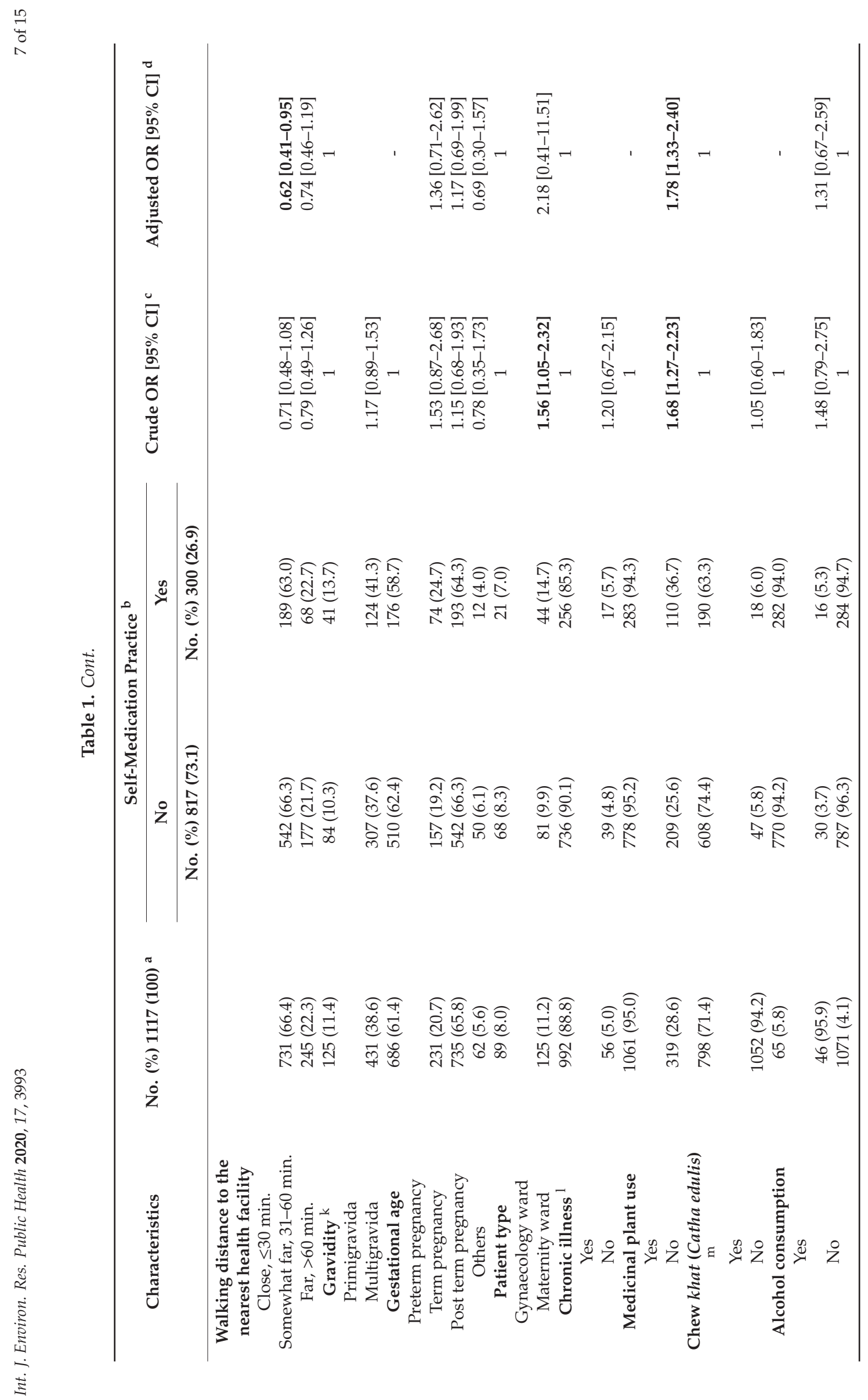




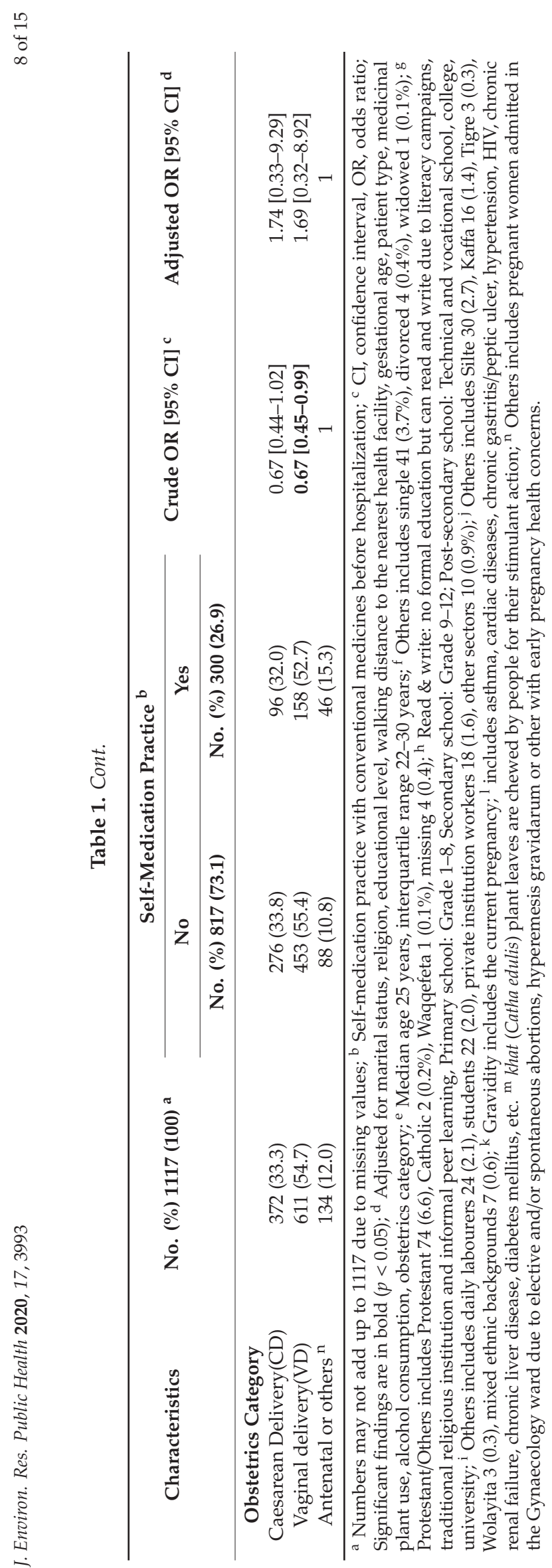


Table 2. Self-medication practice among pregnant women prior to Jimma University Medical Center (JUMC) admission and modified medicine risk classification, Ethiopia, February to June 2017.

\begin{tabular}{|c|c|c|c|}
\hline Therapeutic Class and INN $(N=300)$ & $n(\%)^{\mathrm{a}}$ & ATC Code & Risk Category $^{b}$ \\
\hline \multicolumn{4}{|l|}{ Analgesics $(N=277)$} \\
\hline Paracetamol & $218(72.7)$ & N02BE01 & Probably safe \\
\hline Diclofenac & $33(11.0)$ & M01AB05 & Potentially risky \\
\hline Antipain medicines ${ }^{c}$ & $26(8.7)$ & - & Undetermined \\
\hline Ibuprofen & $9(3.0)$ & M01AE01 & Potentially risky \\
\hline Tramadol & $2(0.7)$ & N02AX02 & Probably safe \\
\hline \multicolumn{4}{|l|}{ Antibacterial $(N=20)$} \\
\hline Amoxicillin & $17(5.7)$ & J01CA04 & Probably safe \\
\hline Cloxacillin & $1(0.3)$ & J01CF02 & Probably safe \\
\hline Metronidazole & $1(0.3)$ & J01XD01 & Probably safe \\
\hline Antibacterial medicines ${ }^{\mathrm{c}}$ & $1(0.3)$ & - & Undetermined \\
\hline \multicolumn{4}{|l|}{ GI medicines $(N=13)$} \\
\hline Antacid ${ }^{\mathrm{C}}$ & $10(3.3)$ & - & Undetermined \\
\hline Omeprazole & $1(0.3)$ & A02BC01 & Potentially risky \\
\hline Metoclopramide & $1(0.3)$ & A03FA01 & Probably safe \\
\hline Hyoscine butylbromide & $1(0.3)$ & A03BВ01 & Probably safe \\
\hline Magnesium sulphate & $1(0.3)$ & A06AD04 & Potentially risky \\
\hline \multicolumn{4}{|l|}{ Antihelmenthics $(N=3)$} \\
\hline Anthelminthic medicines ${ }^{c}$ & $3(1.0)$ & - & Undetermined \\
\hline \multicolumn{4}{|l|}{ Supplements $(N=2)^{\mathrm{d}}$} \\
\hline Ferrous sulphate & $1(0.3)$ & B03АA07 & Probably safe \\
\hline Multivitamin Tablets & $1(0.3)$ & A11AA05 & Probably safe \\
\hline \multicolumn{4}{|l|}{ Antihypertensives $(N=1)$} \\
\hline Hydralazine & $1(0.3)$ & C02DB02 & Potentially risky \\
\hline \multicolumn{4}{|l|}{ Other classes $(N=3)$} \\
\hline Name forgotten Medicines e & $3(1.0)$ & - & Undetermined \\
\hline $\begin{array}{l}\text { a Percentage may exceed } 100 \% \text { due to } \\
\text { self-medicated with conventional medic } \\
\text { based on modified US-FDA and AU-TGA } \\
\text { class "undetermined" was added for medi } \\
\text { not remember the exact names of each mec } \\
\text { are included in the List of Drugs for Ethiop } \\
\text { either the reason for use or the exact names } \\
\text { Administration, US FDA United States Fo } \\
\text { INN International Non-proprietary Name }\end{array}$ & $\begin{array}{l}\text { ole responses } \\
\text { a denominat } \\
\text { ategory; In ac } \\
\text { hose exact na } \\
\text { d In Ethiopia } \\
\text { E), 5th edn., } 2 \\
\text { medicine. Al } \\
\text { d Drug Adm }\end{array}$ & $\begin{array}{l}\text { entage is calc } \\
\text { b Medicine } \\
\text { le three modifi } \\
\text { nen did not rer } \\
\text { lements are cor } \\
\text { en used a medi } \\
\text { : AU-TGA Aus } \\
\text { ATC Anatomic }\end{array}$ & $\begin{array}{l}\text { aking those who } \\
\text { classification was } \\
\text { y classes, a fourth } \\
\text { c The women did } \\
\text { as medicines and } \\
\text { did not remember } \\
\text { Therapeutic Goods } \\
\text { apeutic Chemical, }\end{array}$ \\
\hline
\end{tabular}

\subsection{Factors Associated with Self-Medication Practice during Pregnancy}

Women who used medicinal plants in the current pregnancy (adjusted OR 1.78; 95\% CI 1.33, 2.40) and Islam (adjusted OR 2.22; 95\% CI 1.19, 4.17) or Orthodox Christian (adjusted OR 2.04; 95\% CI 1.06, 3.92) religion followers were more likely to practice self-medication during pregnancy than Protestant Christians and other religious groups. On the other hand, women who had access to a health facility near their place of residence (adjusted OR 0.62; 95\% CI 0.41, 0.95) were less likely to employ self-medication practice during pregnancy $(p<0.05)$. In univariate analysis, self-medication practice during pregnancy was also strongly associated with educational level, obstetrics category and patient type; however, this was not maintained in multivariable logistic regression analysis (Table 1). Other characteristics and pregnancy outcomes like gravidity, parity, gestational age, patient type, delivery route (obstetrics category) and length of hospital stay were not associated with self-medication.

\subsection{Summary of Safety Classification of Medicines Used in Pregnancy}

As shown in Table 2, using the US FDA/AU-TGA classification method, 243 (73.4\%) of the pharmaceuticals used for self-medication were classified as probably safe to use during pregnancy, 
most commonly paracetamol 218 (65.9\%). A total of 45 (13.6\%) medicines were classified as potentially risky to use during pregnancy, mainly diclofenac $33(10.0 \%)$, followed by ibuprofen $9(2.7 \%)$. While there was no medicine in which no classification was available, there were $43(13.0 \%)$ safety "undetermined" medicines because women did not remember the names of the medicines used.

\subsection{Social Drugs Used among Pregnant Women}

The prevalence of at least one substance use among pregnant women (either khat chewing or alcohol drinking) was $9.7 \%$. Around five percent of the pregnant women consumed alcohol during the current pregnancy, mainly local beer (73.9\%). Surprisingly 31 women daily drank variable amounts of alcohol. The proportion of khat chewers was around $6.0 \%$, and $97.0 \%$ of them chewed daily and almost half of them chewed over $166.0 \mathrm{~g}$ of khat every day. None of the pregnant women was an active tobacco smoker (Table 3).

Table 3. Social drugs used among pregnant women prior to admission to JUMC, Ethiopia, February to June 2017.

\begin{tabular}{|c|c|c|}
\hline Characteristics & Frequency & Percent $^{a}$ \\
\hline \multicolumn{3}{|l|}{ Smoke tobacco $(N=1117)$} \\
\hline Yes & 0 & 0 \\
\hline No & 1117 & 100 \\
\hline \multicolumn{3}{|l|}{ Drink alcohol $(N=1117)$} \\
\hline Yes & 46 & 4.1 \\
\hline No & 1071 & 95.9 \\
\hline \multicolumn{3}{|l|}{ Type of alcohol $(N=46)$} \\
\hline Tella (Local beer) & 34 & 73.9 \\
\hline Beer & 12 & 26.1 \\
\hline Wine & 7 & 15.2 \\
\hline Katikala ('Ethiopian vodka') & 4 & 8.7 \\
\hline Type not given/indicated & 1 & 2.2 \\
\hline \multicolumn{3}{|l|}{ Amount of alcohol consumed $(N=46)$} \\
\hline Regular or daily use of variable amounts & 31 & 67.4 \\
\hline Irregular use of variable amounts & 15 & 32.6 \\
\hline \multicolumn{3}{|l|}{ Chew khat $(N=1117)$} \\
\hline Yes & 65 & 5.8 \\
\hline No & 1052 & 94.2 \\
\hline \multicolumn{3}{|l|}{ Length of years chewed $(N=65)$} \\
\hline$\leq 5$ & 29 & 44.6 \\
\hline$>5$ & 36 & 55.4 \\
\hline \multicolumn{3}{|l|}{ Amount chewed daily $(N=65)^{\mathrm{b}}$} \\
\hline One zurba & 21 & 32.3 \\
\hline Two thirds of a zurba & 11 & 16.9 \\
\hline$\leq$ One half of a zurba ${ }^{c}$ & 33 & 50.8 \\
\hline
\end{tabular}

a Percentage may exceed $100 \%$ due to multiple responses, ${ }^{b}$ Women reported in terms of a local measurement unit, the "zurba"; the approximate weight of one zurba khat plant leaf $\approx 250 \mathrm{~g}$. " Two of them chewed varying amounts and infrequently.

\subsection{Factors Associated with Use of Social Drugs during Pregnancy}

Women who used medicinal plants during pregnancy (adjusted OR 2.75; 95\% CI 1.79, 4.24), women admitted to the gynaecology ward (adjusted OR 2.81; 95\% CI 1. 31, 6.04) and Islam (adjusted OR 3.79; 95\% CI 1.13, 12.76) or Orthodox Christian (adjusted OR 4.08; 95\% CI 1.19, 13.99) religion followers were more likely to have used social drugs during pregnancy than their counterparts. On the other hand, women who had shorter hospital stays were less likely to have used social drugs during pregnancy (adjusted OR 0.63; 95\% CI 0.41, 0.95) $(p<0.05)$ (Table S2). 


\section{Discussion}

To the best of our knowledge, this is the first study to investigate self-medication and the safety profiles of medicines used during pregnancy among hospitalized women using both the US FDA and the AU-TGA pregnancy risk classification systems in Ethiopia. This study found that over a quarter $(27.0 \%)$ of mothers self-medicated with at least one type of conventional medicine, mostly analgesics (92.7\%), at some stage of the current pregnancy. A very concerning finding is that (6.7\%) of the self-medications were with antibacterials. Moreover, (36.7\%) of the self-medicated women took at least one medicinal plant concomitantly during pregnancy. Self-medication among pregnant women was found to be influenced by several factors such as walking distance to the nearest health facility, medicinal plant use and religion. Besides, one in ten of the study participants used at least one social drug in pregnancy.

The self-medication rate in our study was comparable to some previous studies $[12,26]$ but larger than other studies $[7,11,25]$ from Ethiopia. This discrepancy in prevalence could emanate from different reasons. First, in this study women were requested to recall self-medication over a nine month period, whereas in many other studies the recall period was limited to two weeks. Second, participants in this study were hospitalized women (most in their third trimester), whereas the women in previous studies were outpatients (most in early stage of pregnancy). Third, the two previous studies with lower prevalence $[7,11]$ were from the capital of Ethiopia, Addis Ababa, which is supposed to have better access to modern health care services and better knowledge, resulting in reduced self-medication rate, compared to its rural counterparts. Finally, the variation in the study settings, methodology, restriction policies on dispensing medicines and disease distribution may partly explain the disparity in the reported magnitude of self-medication.

The worrisome finding in this study is that almost one in fifteen women were using antimicrobials for self-medication. Antibiotic self-medications have been reported in many parts of Africa, Asia, Europe, North and South America [32] at varying rates showing that self-medication with antimicrobials is a global challenge. While responsible self-medication is an important response to medical conditions, self-treatment with antimicrobials is associated with the risk of irrational medicine use, which predisposes patients to antimicrobial resistance and shrinks the range of effective antimicrobials [14,32]. In Ethiopia, where there is low literacy, weak regulation of use of medicine, weak enforcement of regulation and less strict dispensing policies, the dangers posed by antimicrobial resistance to the gestating women, the foetus and the society are immense, demonstrating that rational medicine use practices should be a priority agenda.

Owing to the large quantity of compounds present in medicinal plants, on top of potential inherent toxicity, many different interactions can occur with concomitant use of other pharmaceuticals, which further jeopardizes maternal and foetal safety [33]. The reported rate of concomitant use of medicinal plants with pharmaceuticals during pregnancy in this study $(10.0 \%)$ falls within the range 2.4-77.3\% reported in literature among African pregnant women [2]. Methodological inconsistencies in capturing concomitant use and variations in the study setting, study participants and the definition of what "medicinal plant" encompasses may be the reasons for the differences. As we could only describe co-use and not assess interactions in the current study, there is still a need for future research on the potential clinical implications of interactions for women using both treatment modalities in pregnancy.

Women who used medicinal plants during the current pregnancy were almost twice as likely to self-medicate sometime during pregnancy than non-users. This corroborates previous studies [2,8], which claimed that many pregnant women self-care with medicinal plants first and only seek professional health services when the situation worsens. Such customs may delay proper medical care seeking and possible interaction between plant remedies and conventional medicine, which could be detrimental to maternal and neonatal health.

Gestating women who had a health facility in their neighbourhood were $38 \%$ less likely to self-medicate during pregnancy. The identification of a nearby location of health facilities as a key factor discouraging self-medication during pregnancy may be particularly important in urban and 
peri-urban areas of Ethiopia (like in our study in which more than half were urban residents), where functional access roads and transport services are often in better supply [3,34]. A sizeable proportion of pregnant women may therefore seek proper medical care at nearby health facilities rather than resorting to self-medication to meet their perceived health needs.

In agreement with a study from Eritrea [35] a significant difference was observed in self-medication practice among religious groups, for no known reason. Thus, a topic for future research could be to uncover any underlying factors.

Compared to previous studies $[15,16]$, it is reassuring that a significantly larger proportion of women used medicines categorized as safe to use in pregnancy. On the other hand, $13.6 \%$ of the self-medicated women used potentially risky medications; however, this result is still promising and lower than findings from previous studies $[7,15,16]$. Differences in use of medicine between studies with respect to safety classification may be attributed to disparities in the study design, geography, variables used and size, pregnancy type, demographic characteristics, variety of medicines on the market and their availability to pregnant women. Nevertheless, the results of the present study still revealed that segments of women were exposed to potentially risky medicines, warranting closer attention.

Concerning the safety of medicinal plants used by women, research indicates that flaxseed use in the last two trimesters of pregnancy is associated with increased risk of premature birth, warranting cautious use [36]. On the other hand, Zingibil (Ginger) use is not known to have detrimental effects on the foetus [2].

Our study disclosed that almost all chewers chewed at least $125.0 \mathrm{~g}$ khat daily and had been chewing for a long time (Table 3). As khat has a known appetite suppression effect [37,38], it could lead to a poor nutritional status of the mother and inadequate weight gain during pregnancy, resulting in low birth weight. In addition, khat is associated with constipation [37]; coupled with the constipation effect of iron supplement, khat may hamper treatment adherence and further jeopardize the health of the foetus and the mother. Considering its harmful health impact, khat chewing should be discouraged in pregnancy through appropriate educational intervention.

\section{Study Strengths and Limitations}

This study has several strengths. The main strength is the large sample size of 1117 women that provides greater insight into the used medicines that might have the potential for foetal harm. Equally, health professionals in the study area with knowledge about the healthcare system, local language, culture and previous research or practice experience collected the data. This study had some limitations as well. It was conducted in a tertiary care hospital in Ethiopia and, therefore, this may not be representative of the pregnant female population who accessed primary or secondary care services. Medicine exposure information was collected by relying on the women's recall from their entire pregnancy; therefore, there is a possibility of recall bias leading to underestimation of medicine use among women. Another limitation is that the women may not remember the names of medicines used, which obstructs safety class categorization. Additionally, aborting women were included in the study and their decision to receive an abortion may have influenced their self-medication selection. Finally, since the study was institutional, pregnant women might be confused or embarrassed about disclosing their use of medicines.

\section{Conclusions}

Overall, approximately three out of ten pregnant women self-medicated with at least one type of conventional medicine, mainly analgesics, in this Ethiopian setting. It is reassuring that the majority of women self-medicated with medicines probably safe to use during pregnancy. However, it is also concerning that some women used potentially risky medicines or concomitantly self-cared with medicinal plants. Access to health facility, medicinal plant use and Islam or Orthodox Christian religion were factors associated with self-medication. The findings suggest that there is need for educating pregnant women on the types of illnesses that can be self-diagnosed and self-treated, and the types of 
medicines to be used for self-care in pregnancy to promote responsible self-medication and prevent foetal and maternal risks. In addition, there is a need to raise awareness among women of the fact that even over-the-counter medicines do require advice and counselling from health personnel. Finally, health facilities also need to routinely include pregnant women self-medication history in patient medication records to prevent potential harms of self-medication.

Supplementary Materials: The following are available online at http://www.mdpi.com/1660-4601/17/11/3993/s1. Table S1: Medicinal plants used along with self-medication among pregnant women prior to admission to JUMC, Ethiopia; Table S2: Characteristics of pregnant women according to social drug use prior to admission to JUMC, Ethiopia.

Author Contributions: S.M.A. conceived the idea for the study and its design, collected, analysed, interpreted the data, and wrote the first draft of the manuscript. Y.A.A. and J.S. participated in study coordination. S.M.A., J.S. and F.A. revised and finalized the manuscript. S.M.A., J.S., F.A. and Y.A.A. critically reviewed the manuscript and contributed intellectual content. All authors have read and agreed to the published version of the manuscript.

Funding: S.M.A. was a PhD student at the University of Oslo during the study. He was a recipient of a scholarship from the Norwegian Loan Fund (Lånekassen). He also received a travel grant from Norwegian PhD School in Pharmaceutical Sciences for the data collection. These institutes had no role in the design of the study or the collection, analysis, and interpretation of data or in writing the manuscript.

Acknowledgments: Many thanks must first go to pregnant women admitted at JUMC for generously sharing with us information about their self-medication and social drug use experiences. Special thanks go to the data collectors who skilfully collected the data. The authors would like to acknowledge Norwegian PhD School in Pharmaceutical Sciences for the travel grant assistance for the data collection. A word of thanks is also extended to Ibrahimu Mdala for assistance with data analysis. The authors are truly grateful to Nancy Lea Eik-Nes for editing the manuscript.

Conflicts of Interest: The authors declare that they have no competing interest.

\section{References}

1. Abduelkarem, A.R.; Mustafa, H. Use of Over-the-Counter Medication among Pregnant Women in Sharjah, United Arab Emirates. J. Pregnancy 2017, 2017, 1-8. [CrossRef]

2. Ahmed, Y.; Nordeng, H.; Sundby, J.; Aragaw, Y.A.; De Boer, H.J. The use of medicinal plants by pregnant women in Africa: A systematic review. J. Ethnopharmacol. 2018, 224, 297-313. [CrossRef]

3. Owoseni, J. Determinants of self-medication among pregnant women in Ekiti State, Nigeria. JOHESI 2017, 6, 148-154.

4. Yusuff, K.; Omarusehe, L.-D. Determinants of self-medication practices among pregnant women in Ibadan, Nigeria. Int. J. Clin. Pharm. 2011, 33, 868-875. [CrossRef] [PubMed]

5. Pregnancy care. Health Educ. J. 1985, 44, 158-159. [CrossRef]

6. Ebrahimi, H.; AtashSokhan, G.; Amanpour, F.; Hamidzadeh, A. Self-medication and its risk factors among women before and during pregnancy. Pan Afr. Med. J. 2017, 27, 27-183. [CrossRef] [PubMed]

7. Beyene, K.G.; Worku, S. Self-medication practice and associated factors among pregnant women in Addis Ababa, Ethiopia. Trop. Med. Health 2018, 46, 10. [CrossRef] [PubMed]

8. Agyei-Boateng, R. Self-Medication Practices among Pregnant Women in Ejisu-Juaben Municipality. Ph.D. Thesis, Kwame Nkrumah University of Science and Technology, Kumasi, Ghana, 2015. Available online: http://ir.knust.edu.gh/xmlui/handle/123456789/8137 (accessed on 14 May 2019).

9. Ruiz, M.E. Risks of self-medication practices. Curr. Drug Saf. 2010, 5, 315-323. [CrossRef]

10. Abasiubong, F.; Bassey, E.A.; Udobang, J.A.; Akinbami, O.S.; Udoh, S.B.; Idung, A.U. Self-Medication: Potential risks and hazards among pregnant women in Uyo, Nigeria. Pan Afr. Med. J. 2012, 13, 13-15.

11. Kebede, B.; Gedif, T.; Getachew, A. Assessment of drug use among pregnant women in Addis Ababa, Ethiopia. Pharmacoepidemiol. Drug Saf. 2009, 18, 462-468. [CrossRef]

12. Jambo, A.; Mengistu, G.; Sisay, M.; Amare, F.; Edessa, D. Self-Medication and Contributing Factors Among Pregnant Women Attending Antenatal Care at Public Hospitals of Harar Town, Ethiopia. Front. Pharmacol. 2018, 9. [CrossRef] [PubMed]

13. Mothupi, M. Use of herbal medicine during pregnancy among women with access to public healthcare in Nairobi, Kenya: A cross-sectional survey. BMC Complement. Altern. Med. 2014, 14, 432. [CrossRef] [PubMed] 
14. Sisay, M.; Mengistu, G.; Edessa, D. Epidemiology of self-medication in Ethiopia: A systematic review and meta-analysis of observational studies. BMC Pharmacol. Toxicol. 2018, 19, 56. [CrossRef] [PubMed]

15. Rouamba, T.; Valea, I.; Bognini, J.D.; Kpoda, H.; Mens, P.F.; Gomes, M.F.; Tinto, H.; Kirakoya-Samadoulougou, F. Safety Profile of Drug Use During Pregnancy at Peripheral Health Centres in Burkina Faso: A Prospective Observational Cohort Study. Drugs—Real World Outcomes 2018, 5, 193-206. [CrossRef]

16. Trønnes, J.N.; Lupattelli, A.; Nordeng, H. Safety profile of medication used during pregnancy: Results of a multinational European study ${ }^{\dagger}$. Pharmacoepidemiology and Drug Safety 2017, 26, 802-811. [CrossRef]

17. Nordeng, H. Drug utilization in pregnant women. In: Drug Utilization Research: Methods and Applications, 1st ed.; Elseviers, M., Wattermark, B., Almarsdóttir, A.B., Andersen, M., Benko, R., Bennie, M., Eriksson, I., Godman, B., Krska, J., Poluzzi, E., et al., Eds.; John Wiley \& Sons Ltd.: Chichester, UK, 2016; pp. 240-247.

18. Fekade, M. Alcohol use and associated factors during pregnancy at Kolfe Keraniyo sub city in Addis Ababa, Ethiopia. Ph.D. Thesis, Addis Ababa University, College of Health science, School of Public Health, Addis Ababa, Ethiopia, 2017. Available online: http://etd.aau.edu.et/handle/123456789/14326 (accessed on 2 June 2020).

19. Popova, S.; Lange, S.; Probst, C.; Shield, K.D.; Kraicer-Melamed, H.; Ferreira-Borges, C.; Rehm, J. Actual and predicted prevalence of alcohol consumption during pregnancy in the WHO African Region. Trop. Med. Int. Health 2016, 21, 1209-1239. [CrossRef]

20. Mohammed, M.A.; Bushra, A.W.; Aljadhey, H.S.; Ahmed, J.H.; Grignolo, A. Supplement Use Among Pregnant Women in Ethiopia. Ther. Innov. Regul. Sci. 2013, 47, 416-423. [CrossRef]

21. Mekuriaw, B.; Belayneh, Z.; Yitayih, Y. Magnitude of Khat use and associated factors among women attending antenatal care in Gedeo zone health centers, southern Ethiopia: A facility based cross sectional study. BMC Public Health 2020, 20, 1-8. [CrossRef]

22. Aderaw, A. Caffeine, Alcohol, Khat, and Tobacco use during pregnancy in Butajira, south central Ethiopia. Ph.D. Thesis, Addis Ababa University, College of Health science, School of Public Health, Addis Ababa, Ethiopia, 2018. Available online: http://etd.aau.edu.et/handle/123456789/14442 (accessed on 19 September 2019).

23. Assefa, Y.; Van Damme, W.; Williams, O.D.; Hill, P.S. Successes and challenges of the millennium development goals in Ethiopia: Lessons for the sustainable development goals. BMJ Glob. Health 2017, 2, e000318. [CrossRef]

24. Nakajima, M.; Jebena, M.G.; Taha, M.; Tesfaye, M.; Gudina, E.K.; Lemieux, A.; Hoffman, R.; Al'Absi, M. Correlates of khat use during pregnancy: A cross-sectional study. Addict. Behav. 2017, 73, 178-184. [CrossRef]

25. Befekadu, A.; Dhekama, N.H.; Mohammed, M.A. Self-medication and Contributing Factors among Pregnant Women Attending Antenatal Care in Ethiopia: The Case of Jimma University Specialized Hospital. Med. Sci. Int. Med. J. 2014, 3, 1. [CrossRef]

26. Abeje, G.; Admasie, C.; Wasie, B. Factors associated with self medication practice among pregnant mothers attending antenatal care at governmental health centers in Bahir Dar city administration, Northwest Ethiopia, a cross sectional study. Pan Afr. Med. J. 2015, 20, 276. [CrossRef] [PubMed]

27. Jimma University Specialized hospital. Available online: http://www.ju.edu.et/jimma-university-specializedhospital-jush (accessed on 21 July 2019).

28. Segni, H.; Ayana, D.; Jarso, H. Prevalence of Hyperemesis Gravidarum and Associated Factors Among Pregnant Women at Jimma University Medical Center, South West Ethiopia: A Cross-Sectional Study. EC Gynaecol. 2016, 3, 376-387.

29. Kish, L. Survey Sampling; John Wiley \& Sons Ltd.: New York, NY, USA, 1965.

30. US FDA Pregnancy and Lactation Labeling (Drugs) Final Rule. Available online: https://www.fda.gov/ drugs/labeling-information-drug-products/pregnancy-and-lactation-labeling-drugs-final-rule (accessed on 16 September 2019).

31. Australian TGA: Australian categorisation system for prescribing medicines in pregnancy. Available online: https://www.tga.gov.au/australian-categorisation-system-prescribing-medicines-pregnancy (accessed on 19 September 2019).

32. Limaye, D.; Fortwengel, G.; Limaye, V.; Krause, G. A Systematic Review of the Literature to Assess Self-medication Practices. Ann. Med. Health Sci. Res. 2017, 7, 1-15.

33. Cordier, W.; Steenkamp, V. Drug interactions in African herbal remedies. Drug Metab. Drug Interact. 2011, 26, 53-63. [CrossRef] 
34. Melese, A.G.T.; Zegeye, A.G.A.T.M.K. The Role of Geographical Access in the Utilization of Institutional Delivery Service in Rural Jimma Horro District, Southwest Ethiopia. Prim. Health Care Open Access 2014, 4. [CrossRef]

35. Tesfamariam, S.; Anand, I.S.; Kaleab, G.; Berhane, S.; Woldai, B.; Habte, E.; Russom, M. Self-medication with over the counter drugs, prevalence of risky practice and its associated factors in pharmacy outlets of Asmara, Eritrea. BMC Public Health 2019, 19, 159. [CrossRef]

36. Moussally, K.; Bérard, A. Exposure to herbal products during pregnancy and the risk of preterm birth. Eur. J. Obstet. Gynecol. Reprod. Boil. 2010, 150, 107-108. [CrossRef]

37. Abate, K. Khat (Catha edulis) chewing as a risk factor of low birth weight among full term Newborns: A systematic review. Middle East J. Fam. Med. 2015, 13, 10-14.

38. Dendir, E.; Deyessa, N. Substance use and birth weight among mothers attending public hospitals: A case control study. Ethiop. J. Health Dev. 2017, 31, 27-35.

(C) 2020 by the authors. Licensee MDPI, Basel, Switzerland. This article is an open access article distributed under the terms and conditions of the Creative Commons Attribution (CC BY) license (http://creativecommons.org/licenses/by/4.0/). 



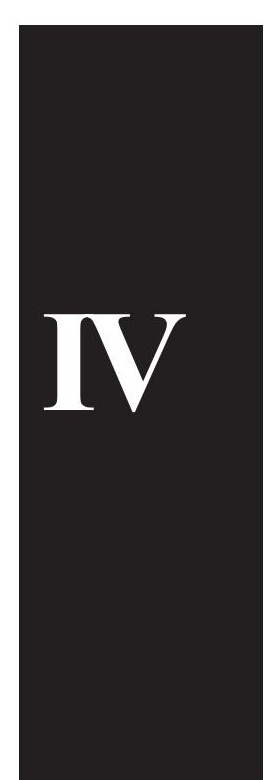





\title{
Medication-related problems among hospitalized pregnant women in a tertiary teaching hospital in Ethiopia: a prospective observational study
}

Seid Mussa Ahmed ${ }^{1,2^{*}}$ (D) Johanne Sundby ${ }^{1}$, Yesuf Ahmed Aragaw ${ }^{3}$ and Hedvig Nordeng ${ }^{4,5}$

\begin{abstract}
Background: Studies on medication-related problems (MRPs) among pregnant women are scarce, despite the potential consequences for both mother and child. This study aimed to describe the prevalence, clinical significance, and risk factors for MRPs among hospitalized pregnant or postpartum women at Jimma University Medical Centre (JUMC) in Ethiopia.

Methods: A prospective follow-up and clinical audit of 1117 hospitalized pregnant or postpartum women in the maternity and gynaecology wards at JUMC was carried out between February and June 2017. Patients were followed throughout their stay in the hospital to assess the presence and development of MRPs. Pre-tested data extraction form and an interview-guided structured questionnaire were used to collect data. Descriptive statistics were used to describe MRPs. Logistic regression analysis was used to identify factors associated with MRPs.

Results: One or more MRPs occurred among 323 (28.9\%) study participants, mostly in relation to lack of iron supplementation. A total of 278 (70.6\%) of all MRPs were considered to be of moderate to high clinical significance. When excluding MRPs due to iron from the analysis, chronic disease (adjusted OR 1.91; 95\% Cl 1.02, 3.58), medication use prior to admission (adjusted OR 2.38; 95\% Cl 1.24, 4.56), nulliparity (adjusted OR 1.99; 95\% Cl 1.22, 3.24) and multiparity (adjusted OR $1.91 ; 95 \% \mathrm{Cl} 1.17,3.12$ ) were significantly associated with experiencing an MRP.

Conclusions: Nearly 3 out of 10 hospitalized pregnant women at JUMC had one or more MRPs. The need for additional iron therapy was by far the most common type of MRP. Improved adherence to guidelines on iron supplementation are required. Multidisciplinary approaches including physicians, nurses, anesthesia professionals and clinical pharmacists in the maternity and gynaecology wards could possibly prevent MRPs and promote patient safety for women and children.
\end{abstract}

Keywords: Medication-related problem, Maternity, Gynaecology, Pregnancy, Clinical significance, Iron supplementation

\footnotetext{
* Correspondence: seidma@studmed.uio.no

${ }^{1}$ Department of Community Medicine and Global Health, Institute of Health and Society, Faculty of Medicine, University of Oslo, Oslo, Norway

${ }^{2}$ Division of Social and Administrative Pharmacy, School of Pharmacy, Faculty

of Health Sciences, Jimma Institute of Health, Jimma University, Jimma,

Ethiopia

Full list of author information is available at the end of the article
}

(C) The Author(s). 2020 Open Access This article is licensed under a Creative Commons Attribution 4.0 International License, which permits use, sharing, adaptation, distribution and reproduction in any medium or format, as long as you give appropriate credit to the original author(s) and the source, provide a link to the Creative Commons licence, and indicate if changes were made. The images or other third party material in this article are included in the article's Creative Commons licence, unless indicated otherwise in a credit line to the material. If material is not included in the article's Creative Commons licence and your intended use is not permitted by statutory regulation or exceeds the permitted use, you will need to obtain permission directly from the copyright holder. To view a copy of this licence, visit http://creativecommons.org/licenses/by/4.0/. The Creative Commons Public Domain Dedication waiver (http://creativecommons.org/publicdomain/zero/1.0/) applies to the data made available in this article, unless otherwise stated in a credit line to the data. 


\section{Background}

A medication-related problem (MRP) is defined as an unwanted event or circumstance involving medication therapy that actually or potentially interferes with desired health outcomes [1,2]. Studies have shown that the prevalence of MRPs among hospitalized pregnant patients varies from 42 to $83 \%[3,4]$. Patients who have MRPs are likely to have a longer hospital stay, recurrent hospital admissions, reduced quality of life, increased overall health care cost, and even an increased risk of morbidity and mortality [4-6].

Only a handful of studies have examined the frequency and nature of MRPs occurring in an obstetric hospital inpatient setting [3, 4]. A recently published Norwegian study of 212 pregnant women in an inpatient setting identified 105 MRPs occurring in $42 \%$ of pregnant women. "Need for additional drug" (46.7\%), "adverse drug reaction" (20.0\%), and "patient adherence" (10.5\%) were the most common categories of MRPs. The most common medication groups involved in the MRPs were drugs acting on the respiratory system (25\%; mainly nasal decongestants, 9\%), anti-infectives for systemic use (18\%; mainly antibiotics for systemic use, $8 \%$ ), and drugs acting on blood and blood-forming organs (16\%; mainly iron supplementation, 14\%) [4]. A study from Australia identified 400 potential MRPs in 171 of 205 hospitalized pregnant women. The majority of MRPs were of low clinical significance (92\%). The most common types of MRPs were "incomplete medications charted on admission" (28\%), "dose too high" (26\%), "incomplete drug order" (15\%), and "additional medication required" (13\%). The therapeutic groups most commonly associated with MRPs were medications for the alimentary tract and metabolism, mainly aperients (18\%) and vitamins (13\%), followed by drugs for the nervous system, mainly analgesics (25\%) and antidepressants (4\%) [3]. In addition to these two studies, a few studies have evaluated medication errors in obstetric and maternity wards [7-9].

In Ethiopia, previous studies have focused mainly on prescription drug use, drug use patterns, and selfmedication practices among pregnant outpatients attending obstetrics and gynaecology departments [10-13]. Notably, none of these prior investigations involved the identification of MRPs and were conducted in ambulatory pregnant patients. Although few MRPs identification studies were performed among hospitalized patients in the country, all focused on the non-pregnant patient population [14-17]. Thus far, no study has investigated MRPs in an obstetrics group in a hospital setting in Ethiopia. Therefore, the objective of the present study was to determine the prevalence, clinical significance, and risk factors of MRPs occurring in hospitalized pregnant women in the maternity and gynaecology wards of Jimma University Medical Centre (JUMC) in Southwest Ethiopia.

\section{Methods}

\section{Sample size}

The required sample size for this study was calculated assuming a $50 \%$ proportion of MRPs, $5 \%$ level of precision, 3\% error margin, and 5\% possible non-response rate, making the minimum sample size 1121 pregnant women.

\section{Study setting}

A facility-based prospective observational study was conducted in the maternity and gynaecology wards of JUMC, a tertiary level public teaching hospital located in Jimma City in southwest Ethiopia, $350 \mathrm{~km}$ from the capital city of Addis Ababa. It is the only teaching and tertiary level care hospital in southwest Ethiopia, with a catchment population of approximately 20 million people $[18,19]$. Most of the pregnant women referred to the hospital come from rural areas, where many deliveries are attended at home [20, 21]. The Department of Obstetrics and Gynaecology at JUMC provides specialized health services for approximately 7580 inpatients and 11,590 outpatients each year, with a bed capacity of 265. The department has two wards (gynaecology and maternity/labour), one general gynaecological outpatient clinic, one antenatal care outpatient clinic, and one family planning clinic. Women are treated at the gynaecology inpatient ward before 28 weeks of pregnancy. Most pregnant women admitted to this ward have elective and/or spontaneous abortions, hyperemesis gravidarum (HEG), or other early pregnancy complications. After 28 weeks of pregnancy, women are admitted to the maternity/labour inpatient ward. Women having a vaginal delivery give birth in the labour ward and are transferred to the maternity ward after delivery. If the mother and baby are healthy, they are discharged at the earliest possible time after delivery, usually within 1-2 days. Women having a caesarean delivery are transferred to the maternity ward and usually stay for $72 \mathrm{~h}$.

\section{Data collection and procedures}

Women in the maternity and gynaecology wards at JUMC between February and June 2017 were invited to participate in the study during normal working hours. Patients were informed of the aim and procedures of the study, and written informed consent was obtained from each study participant. Women who were under 18 years of age, too ill to participate, who declined to participate, were hard of hearing, unable to speak or with mental illness, admitted for a brief time $(<4 \mathrm{~h})$, and non-pregnant women admitted to the gynaecology ward were excluded from the study.

The women were followed throughout their stay in the hospital to assess the presence and development of MRPs. Pre-tested data extraction form and an interview- 
guided structured questionnaire were used to collect the data. Five trained clinical pharmacists (data abstraction and MRP assessment) and four trained nurses (the questionnaire) from JUMC collected the data.

Information on the reason for admission, diagnoses, dosage regimens, discharge medications, maternal and perinatal outcomes, laboratory results, and length of hospital stay was collected by reviewing patients' medical cards and medication charts. The card and chart reviews were performed for each patient on the first day of admission and repeated on subsequent days. The questionnaire was used to collect maternal socio-demographic characteristics, obstetric history, past medical history and medication experience, social drug use, and medicinal plant use.

\section{MRP identification and assessment}

MRPs were classified into eight categories: need for additional drug therapy, unnecessary drug therapy, dose too low, dose too high, ineffective drug, adverse drug reactions, noncompliance [1], and other, subdivided into need for additional laboratory test and/or incomplete drug order (Additional file 1) [3].

MRPs were identified by reviewing patients' medical cards and medication charts, and patient interviews about medication use while in the hospital. A panel of experts comprised of senior clinical pharmacists and experienced obstetricians/gynaecologists identified MRPs and classified them into categories as recommended by Cipolle et al. [1] The panel of experts further refined the MRP identification and classification method for the study setting in accordance with Ethiopian standard treatment guidelines and literature reviews (Additional file 1) [3, 22-25].

The clinical significance of each MRP was categorized as level 1 or level 2 [3]. Level 1 are those MRPs that have low potential to give rise to patient discomfort or clinical deterioration whereas level 2 are MRPs that have moderate to severe potential to give rise to patient discomfort or clinical deterioration $[3,26]$. At first, experienced clinical pharmacists identifying MRPs in the wards assessed and classified the clinical significance, and subsequently discussed by the panel of experts. Differences in opinion on severity level of MRPs were discussed until consensus was reached. The description of the MRPs, their clinical significance, and the medication (s) involved were recorded using a purpose-built data collection tool.

The classification of medications involved in MRPs was performed per the World Health Organization (WHO) Anatomical Therapeutic Chemical Classification system (ATC) that categorizes medications into 14 main groups [27].

\section{Statistical analysis}

Descriptive statistics were used to calculate percentages. The results were presented as medians and ranges. Univariate and multivariate logistic regression analyses were used to calculate odds ratios (ORs) with 95\% confidence intervals (CIs) and identify risk factors associated with MRPs. The independent variables were patient-related factors (age, level of education, marital status, occupation, religion, ethnic group, family size, residence place, alcohol use status, and khat chewing), disease-related factors (patient admission ward, i.e., gynaecology or maternity ward; chronic disease; obstetrics category, i.e., caesarean or vaginal delivery; duration of hospital stay), pregnancy related factors (parity, gravidity, gestational age, adverse pregnancy outcome [current and previous], status of anaemia), medicine-related factors (medicines used during admission or prior to admission, ferrous sulphate supplementation, medicinal plant use, concomitant use of medicinal plants), facility-related factors (walking distance to the nearest health facility, and availability of preferred medication for a specific condition). Explanatory variables with $p \leq 0.05$ in the univariate analysis were entered into a multivariate logistic regression model to determine independent risk factors of MRPs. As iron supplements were involved in 165 (41.9\%) of the MRPs, a post hoc logistic regression analysis was performed excluding iron. All data were analysed using the Statistical Package for the Social Sciences (SPSS) software version 25.0 for Windows (IBM ${ }^{\circledR}$ SPSS $^{\bullet}$ Statistics, Armonk).

\section{Results}

\section{Study population characteristics}

A total of 1137 pregnant and nursing women were asked to participate in the study, and 1121 (98.6\%) accepted. Responses from four women were incomplete, leaving 1117 women in the final study population, $88.8 \%$ from the maternity ward (611 vaginal deliveries, 372 caesarean sections, and 9 did not proceed to parturition) and $11.2 \%$ from the gynaecology ward. The median patient age was 25 years (range 18-45 years). Most of the women were either primiparous or multiparous (40\% each). Most women gave birth at term (65.8\%) through vaginal labour (54.7\%). Five percent of the women had one or more chronic diseases. The median length of hospital stay was 3 days, ranging from $5 \mathrm{~h}$ to 60 days, and most of the patients (59.7\%) stayed $\leq 3$ days in the hospital. A fifth of the women (19.4\%) had adverse pregnancy outcomes in the current pregnancy, and 1 in 10 women (11.0\%) had a history of adverse pregnancy outcomes. Detailed sociodemographic characteristics and clinical data are summarized in Tables 1 and 2. In this study, concomitant use of phytomedicines and conventional medicines was assessed by identifying women who used both during pregnancy for the same or different illnesses.

\section{Medicine use during pregnancy and admission}

The majority of the women had used one or more medications during pregnancy $(85.2 \%)$, whereas $28.6 \%$ of 
Table 1 Risk factors of medication-related problems ${ }^{\text {a }}$

\begin{tabular}{|c|c|c|c|c|c|}
\hline \multirow[t]{2}{*}{ Characteristics } & \multirow{2}{*}{$\begin{array}{l}\text { No. (\%) } \\
1117 \text { (100) }\end{array}$} & \multicolumn{2}{|l|}{ MRPs } & \multirow{2}{*}{$\begin{array}{l}\text { Crude OR } \\
(95 \% \mathrm{Cl})\end{array}$} & \multirow{2}{*}{$\begin{array}{l}\text { Adjusted OR } \\
(95 \% \mathrm{Cl})^{\mathrm{b}}\end{array}$} \\
\hline & & No MRPs & $\geq 1 \mathrm{MRP}$ & & \\
\hline \multicolumn{6}{|l|}{ Age (years) } \\
\hline$\leq 20$ & $223(20.0)$ & 169 & 54 & 1 & 1 \\
\hline $21-25$ & $388(34.7)$ & 278 & 110 & $1.24[0.85,1.81]$ & $1.34[0.91,1.98]$ \\
\hline $26-30$ & $320(28.7)$ & 216 & 104 & $1.51[1.03,2.22]$ & $1.63[1.07,2.50]$ \\
\hline$\geq 31$ & $186(16.7)$ & 131 & 55 & $1.31[0.85,2.04]$ & $1.31[0.79,2.19]$ \\
\hline \multicolumn{6}{|l|}{ Residence place } \\
\hline Urban & $595(53.3)$ & 423 & 172 & 1 & \\
\hline Rural & $522(46.7)$ & 371 & 151 & $1.00[0.77,1.30]$ & \\
\hline \multicolumn{6}{|l|}{ Chronic disease } \\
\hline Yes & $56(5.0)$ & 34 & 22 & 1 & \\
\hline No & $1061(95.0)$ & 760 & 301 & $0.61[0.35,1.06]$ & \\
\hline \multicolumn{6}{|l|}{ Medicinal plant used in current pregnancy } \\
\hline Yes & $319(28.6 \%)$ & 228 & 91 & 1 & \\
\hline No & $798(71.4 \%)$ & 566 & 232 & $1.03[0.77,1.37]$ & \\
\hline \multicolumn{6}{|l|}{ Alcohol consumers } \\
\hline Yes & $46(4.1)$ & 33 & 13 & 1 & \\
\hline No & $1071(95.9)$ & 761 & 310 & $1.03[0.54,1.99]$ & \\
\hline \multicolumn{6}{|l|}{ Khat chewers ${ }^{c}$} \\
\hline Yes & $65(5.8)$ & 44 & 21 & 1 & \\
\hline No & $1052(94.2)$ & 750 & 302 & $0.84[0.49,1.44]$ & \\
\hline \multicolumn{6}{|l|}{ No. of medicines during admission } \\
\hline$<5$ medication & $631(57.3)$ & 455 & 176 & 1 & \\
\hline$\geq 5$ medication & $470(42.7)$ & 329 & 141 & $1.11[0.85,1.44]$ & \\
\hline \multicolumn{6}{|l|}{ No. of medicines prior to admission } \\
\hline No past medication & $165(14.8)$ & 119 & 46 & 1 & \\
\hline Only one past medication & $666(59.6)$ & 466 & 200 & $1.11[0.76,1.62]$ & \\
\hline Two or more past medications & $286(25.6)$ & 209 & 77 & $0.95[0.62,1.46]$ & \\
\hline \multicolumn{6}{|l|}{ Duration of hospital stay } \\
\hline$\leq 3$ days & $667(59.7)$ & 482 & 185 & 1 & \\
\hline$>3$ days & $450(40.3)$ & 312 & 138 & $1.15[0.89,1.50]$ & \\
\hline \multicolumn{6}{|l|}{ Gestational age } \\
\hline Preterm pregnancy & $231(20.7)$ & 150 & 81 & 1 & 1 \\
\hline Term pregnancy & $735(65.8)$ & 539 & 196 & $0.67[0.49,0.92]$ & $0.79[0.51,1.23]$ \\
\hline Post term pregnancy & $62(5.6)$ & 46 & 16 & $0.64[0.34,1.21]$ & $0.72[0.36,1.46]$ \\
\hline Others & $89(8.0)$ & 59 & 30 & $0.94[0.56,1.58]$ & $1.04[0.58,1.89]$ \\
\hline \multicolumn{6}{|l|}{ Patient ward } \\
\hline Gynaecology ward & $125(11.2)$ & 78 & 47 & 1 & 1 \\
\hline Maternity ward & $992(88.8)$ & 716 & 276 & $0.64[0.43,0.94]$ & $0.76[0.44,1.30]$ \\
\hline \multicolumn{6}{|l|}{ Adverse pregnancy outcome in the current pregnancy } \\
\hline Yes & $217(19.4)$ & 149 & 68 & 1 & \\
\hline No or not yet delivered and outcome not yet known & $900(80.6)$ & 645 & 255 & $0.87[0.63,1.20]$ & \\
\hline \multicolumn{6}{|l|}{ Previous adverse pregnancy outcome } \\
\hline Yes & $123(11.0)$ & 84 & 39 & 1 & \\
\hline
\end{tabular}


Table 1 Risk factors of medication-related problems a (Continued)

\begin{tabular}{|c|c|c|c|c|c|}
\hline \multirow[t]{2}{*}{ Characteristics } & \multirow{2}{*}{$\begin{array}{l}\text { No. (\%) } \\
1117 \text { (100) }\end{array}$} & \multicolumn{2}{|l|}{ MRPs } & \multirow{2}{*}{$\begin{array}{l}\text { Crude OR } \\
(95 \% \mathrm{Cl})\end{array}$} & \multirow{2}{*}{$\begin{array}{l}\text { Adjusted OR } \\
(95 \% \mathrm{Cl})^{b}\end{array}$} \\
\hline & & No MRPs & $\geq 1 \mathrm{MRP}$ & & \\
\hline No/not Applicable & $994(89.0)$ & 710 & 284 & $0.86[0.58,1.29]$ & \\
\hline \multicolumn{6}{|l|}{ Parity } \\
\hline Primiparous & $227(20.3)$ & 181 & 46 & 1 & 1 \\
\hline Nulliparous & 441 (39.5) & 308 & 133 & $1.70[1.16,2.49]$ & $1.82[1.23,2.69]$ \\
\hline Multiparous & $449(40.2)$ & 305 & 144 & $1.86[1.27,2.72]$ & $1.73[1.16,2.59]$ \\
\hline
\end{tabular}

Bold, statistically significant, $P<0.05$

a Numbers may not add up to $100 \%$ due to missing values

${ }^{\mathrm{b}}$ Adjusted for age, gestational age, patient ward and parity

' Khat (Catha edulis) plant leaves are chewed by people to attain a state of euphoria and stimulation

women (Tables 1 and 2) had used medicinal plants. Ferrous sulphate was the most commonly used medication prior to hospital admission (97.3\%). Furthermore, 271 (24.3\%) women concomitantly used medicinal plants and medicines prior to admission.

During admission, the median number of prescribed medications was 3 per patient (range: 0-24; Additional file 2) and $42.7 \%$ of the participants were taking $\geq 5$ medications (Tables 1 and 2). The three most common types of medications given during the women's hospital stay were pitocin (63.7\%), normal saline (38.9\%), and ceftriaxone (36.0\%) (Additional file 3). Ferrous sulphate $(54.4 \%)$, cephalexin (30.4\%), and metronidazole (25.0\%) were the three most common medications prescribed at hospital discharge (Additional file 4).

\section{Medication use-related problems}

One or more MRPs occurred among $28.9 \%$ of the study participants: $23.7 \%$ had one MRP, $4.2 \%$ had two MRPs, $0.8 \%$ had three MRPs, and $0.2 \%$ had four MRPs. A total of 394 discrete MRPs were noted. The highest number of MRPs, $87.6 \%$, was identified among women admitted in the maternity ward (228 MRPs among those with vaginal delivery, 114 MRPs among caesarean sections, and 3 among those not yet delivered; Table 1, Additional file 2).

Two hundred and seventy-eight (70.6\%) of all MRPs were considered to be of moderate to high clinical significance and classified as level 2 MRPs. One hundred sixty five (41.9\%) of the total MRPs (133 (47.8\%) of level 2 and $32(39.0 \%)$ of level 1 MRPs) were due to iron treatment/supplementation.

Chart reviews were usually performed twice, with a range of one to three reviews (Additional file 2).

In $14.5 \%$ of patients, lack of chart recording or documentation of medication administration occurred, most commonly for ceftriaxone, anaesthetic drugs, and intravenous fluids in relation to caesarean section in the surgical delivery room. The assessment panel of experts agreed not to consider this an MRP, as the medications were appropriately administered to the patients.
The types of MRPs according to the eight main MRP categories are presented in Table 3. The most common MRP types were: need for additional drug therapy $(n=$ 236 cases, 73.1\%), need for an additional laboratory test ( $n=41$ cases, $12.7 \%)$, unnecessary drug therapy $(n=38$ cases, $11.8 \%)$, and too low dosage $(n=38$ cases, $11.8 \%$; Table 3).

A more detailed overview of the specific causes of MRPs and medications involved in MRPs are found in Additional file 5 and Additional file 6, respectively.

As indicated in Fig. 1, the most common therapeutic group implicated in MRPs were medications acting on blood and blood-forming organs, mainly ferrous sulphate (35.3\%), followed by anti-infectives for systemic use, largely cephalexin and metronidazole (each 9.4\%).

\section{Factors contributing to MRPs}

Nulliparous (adjusted OR 1.82; 95\% CI 1.23, 2.69) and multiparous (adjusted OR 1.73; 95\% CI 1.16, 2.59) women were significantly more likely to experience MRP than primiparous women. Similarly, women aged $>26$ years (adjusted OR 1.63; 95\% CI 1.07, 2.50) were more likely to experience MRPs than their counterparts (Table 1). However, in a post hoc analysis excluding MRPs due to iron from the analysis, only parity was maintained as a risk factor. Additional risk factors, including chronic disease (adjusted OR 1.91; 95\% CI 1.02, 3.58) and past medication use (adjusted OR 2.38; 95\% CI 1.24, 4.56; adjusted OR 2.21; $95 \%$ CI 1.12, 4.38) were associated with a significantly increased likelihood of experiencing an MRP than their counterparts (Table 2).

All MRPs were considered clinically significant for the patients. Most commonly, "need for additional medication therapy" included untreated disease conditions, mainly anaemia or an absence of anti-infection prophylaxis, as in patients at risk of infection due to retained placenta not receiving prophylactic antibiotic. Additional file 5 describes in detail the different types and causes of MRPs. 
Table 2 Risk factors of medication-related problems, excluding iron preparations ${ }^{\text {a }}$

\begin{tabular}{|c|c|c|c|c|c|}
\hline \multirow[t]{2}{*}{ Variable category } & \multirow{2}{*}{$\begin{array}{l}\text { No. (\%) } \\
1117(100)\end{array}$} & \multicolumn{2}{|c|}{ Non-Iron MRPs ${ }^{b}$} & \multirow{2}{*}{$\begin{array}{l}\text { Crude OR } \\
(95 \% \mathrm{Cl})\end{array}$} & \multirow{2}{*}{$\begin{array}{l}\text { Adjusted OR } \\
(95 \% \mathrm{Cl})^{c}\end{array}$} \\
\hline & & No MRPs & $\geq 1 \mathrm{MRP}$ & & \\
\hline \multicolumn{6}{|l|}{ Age } \\
\hline$\leq 20$ & $223(20.0)$ & 190 & 33 & 1 & \\
\hline $21-25$ & $388(34.7)$ & 325 & 63 & $1.12[0.71,1.76]$ & \\
\hline $26-30$ & $320(28.7)$ & 259 & 61 & $1.36[0.85,2.16]$ & \\
\hline$\geq 31$ & $186(16.7)$ & 159 & 27 & $0.98[0.56,1.70]$ & \\
\hline \multicolumn{6}{|l|}{ Residence place } \\
\hline Urban & $595(53.3)$ & 493 & 102 & 1 & \\
\hline Rural & $522(46.7)$ & 440 & 82 & $0.90[0.66,1.24]$ & \\
\hline \multicolumn{6}{|l|}{ Chronic disease } \\
\hline No & $1061(95.0)$ & 892 & 169 & 1 & 1 \\
\hline Yes & $56(5.0)$ & 41 & 15 & $1.93[1.05,3.57]$ & $1.91[1.02,3.58]$ \\
\hline \multicolumn{6}{|l|}{ Medicinal plant used in current pregnancy } \\
\hline Yes & $319(28.6 \%)$ & 265 & 54 & 1 & \\
\hline No & $798(71.4 \%)$ & 668 & 130 & $0.96[0.68,1.35]$ & \\
\hline \multicolumn{6}{|l|}{ Alcohol consumers } \\
\hline Yes & $46(4.1)$ & 37 & 9 & 1 & \\
\hline No & $1071(95.9)$ & 896 & 175 & $0.80[0.38,1.69]$ & \\
\hline \multicolumn{6}{|l|}{ Khat chewers ${ }^{d}$} \\
\hline Yes & $65(5.8)$ & 59 & 6 & 1 & \\
\hline No & $1052(94.2)$ & 874 & 178 & $2.00[0.85,4.71]$ & \\
\hline \multicolumn{6}{|l|}{ No. of medicines during admission } \\
\hline$<5$ medication & $631(57.3)$ & 520 & 111 & 1 & \\
\hline$\geq 5$ medication & $470(42.7)$ & 399 & 71 & $0.83[0.60,1.15]$ & \\
\hline \multicolumn{6}{|l|}{ No. of medicines prior to admission } \\
\hline No past medication & $165(14.8)$ & 152 & 13 & 1 & 1 \\
\hline Only one past medication & $666(59.6)$ & 546 & 120 & $2.57[1.41,4.68]$ & $2.38[1.24,4.56]$ \\
\hline Two or more past medications & $286(25.6)$ & 235 & 51 & $2.54[1.34,4.82]$ & $2.21[1.12,4.38]$ \\
\hline \multicolumn{6}{|l|}{ Duration of hospital stay } \\
\hline$\leq 3$ days & $667(59.7)$ & 555 & 112 & 1 & \\
\hline$>3$ days & $450(40.3)$ & 378 & 72 & $0.94[0.68,1.31]$ & \\
\hline \multicolumn{6}{|l|}{ Gestational age } \\
\hline Preterm pregnancy & $231(20.7)$ & 199 & 32 & 1 & \\
\hline Term pregnancy & $735(65.8)$ & 608 & 127 & $1.30[0.85,1.98]$ & \\
\hline Post term pregnancy & $62(5.6)$ & 51 & 11 & $1.34[0.63,2.84]$ & \\
\hline Others & $89(8.0)$ & 75 & 14 & $1.16[0.59,2.30]$ & \\
\hline \multicolumn{6}{|l|}{ Patient ward } \\
\hline Gynaecology ward & $125(11.2)$ & 113 & 12 & 1 & 1 \\
\hline Maternity ward & $992(88.8)$ & 820 & 172 & $1.98[1.07,3.66]$ & $1.34[0.68,3.58]$ \\
\hline \multicolumn{6}{|l|}{ Adverse pregnancy outcome in the current pregnancy } \\
\hline Yes & $217(19.4)$ & 185 & 32 & 1 & \\
\hline No or not yet delivered and outcome not yet known & $900(80.6)$ & 748 & 152 & $1.18[0.78,1.78]$ & \\
\hline \multicolumn{6}{|l|}{ Previous adverse pregnancy outcome } \\
\hline Yes & $123(11.0)$ & 106 & 17 & 1 & \\
\hline
\end{tabular}


Table 2 Risk factors of medication-related problems, excluding iron preparations ${ }^{\text {a }}$ (Continued)

\begin{tabular}{|c|c|c|c|c|c|}
\hline \multirow[t]{2}{*}{ Variable category } & \multirow{2}{*}{$\begin{array}{l}\text { No. (\%) } \\
1117 \text { (100) }\end{array}$} & \multicolumn{2}{|c|}{ Non-Iron MRPs ${ }^{b}$} & \multirow{2}{*}{$\begin{array}{l}\text { Crude OR } \\
(95 \% \mathrm{Cl})\end{array}$} & \multirow{2}{*}{$\begin{array}{l}\text { Adjusted OR } \\
(95 \% \mathrm{Cl})^{\mathrm{c}}\end{array}$} \\
\hline & & No MRPs & $\geq 1 \mathrm{MRP}$ & & \\
\hline No/not Applicable & $994(89.0)$ & 827 & 167 & $1.26[0.74,2.16]$ & \\
\hline \multicolumn{6}{|l|}{ Parity } \\
\hline Primiparous & $227(20.3)$ & 203 & 24 & 1 & 1 \\
\hline Nulliparous & 441 (39.5) & 360 & 81 & $1.90[1.17,3.10]$ & $1.99[1.22,3.24]$ \\
\hline Multiparous & $449(40.2)$ & 370 & 79 & $1.81[1.11,3.94]$ & $1.91[1.17,3.12]$ \\
\hline
\end{tabular}

Bold, statistically significant, $P<0.05$

Abbreviations: OR: odd ratio; Cl: Confidence interval

a Numbers may not add up to $100 \%$ due to missing values

b ${ }^{b}$ MRPs $=$ MRPs due to other medications + Patients with No MRPs. $\geq 1$ MRP $=\geq 1$ MRPs due to iron sulphate

c Adjusted for chronic disease, number of medicines prior to admission, patient ward and parity

${ }^{d}$ Khat (Catha edulis) plant leaves are chewed by people to attain a state of euphoria and stimulation

\section{Discussion}

This study provides new knowledge about the prevalence, clinical significance, risk factors, and medications implicated in MRPs among hospitalized pregnant women in a resource-limited setting. To the best of our knowledge, this is the first study in Ethiopia to investigate the extent of MRPs in hospitalized pregnant women. Approximately 3 out of 10 pregnant women had one or more MRPs, mostly in relation to a lack of iron supplementation. More than 7 out of 10 MRPs were considered to be of moderate to severe clinical relevance. This high magnitude and the frequency of these MRPs suggests problems inherent in the day-to-day practices of the study wards. These problems can potentially be improved through internal audits, improved routines, and systemic changes, including multidisciplinary collaboration, training, and increased staff.

In the present study, nearly one-third of pregnant and nursing women encountered at least one MRP. This is lower than reported in studies of Norwegian [4] and Australian [3] pregnant and lactating inpatients, as 42.0 and $83.4 \%$ of the study participants, respectively, experienced at least one MRP. This large variation in prevalence is likely a reflection of the methodological differences between the studies, especially the process of medication reconciliation and medication chart reviews.

Table 3 Overview of Medication Related Problems (MRP) according to frequency and types

\begin{tabular}{|c|c|c|c|}
\hline MRPs Category & Type of MRP & $n(\%)^{a}$ & Example \\
\hline \multirow[t]{2}{*}{ Indication } & Needs additional drug therapy & $236(73.1)$ & $\begin{array}{l}\text { Patient is asthmatic, but is not getting the recommended drug } \\
\text { i.e. salbutamol puff PRN }\end{array}$ \\
\hline & Unnecessary drug therapy & $38(11.8)$ & $\begin{array}{l}\text { Patient is on ceftriaxone } 1 \mathrm{~g} \text { IV BID but there is no indication } \\
\text { of infection in the diagnosis }\end{array}$ \\
\hline \multirow[t]{2}{*}{ Effectivenes } & Dosage too low & $38(11.8)$ & $\begin{array}{l}\text { Cephalexin } 500 \text { mg once PO daily given to patient to treat infection, } \\
\text { PO BID daily is recommended }\end{array}$ \\
\hline & Ineffective drug product & $12(3.7)$ & $\begin{array}{l}\text { HIV/AIDS (immunocompromised) and MRSA infected patient who } \\
\text { was on wound care was on metronidazole and cephalexin treatment } \\
\text { (less effective), instead patient was put on more effective drug, } \\
\text { vancomycin } 500 \text { mg IV BID for } 10 \text { days }\end{array}$ \\
\hline \multirow[t]{2}{*}{ Safety } & Dosage too high & $12(3.7)$ & $\begin{array}{l}\text { Patient is on ceftriaxone } 2 \mathrm{~g} \mathrm{IV} \mathrm{bid} \mathrm{to} \mathrm{treat} \mathrm{chorioamnionitis} \mathrm{which} \\
\text { is high dose, } 1 \mathrm{~g} \text { IV BID is enough }\end{array}$ \\
\hline & Adverse drug reaction & $2(0.6)$ & $\begin{array}{l}\text { Patient received furosemide and gentamicin concurrently. One increases } \\
\text { toxicity of the other by pharmacodynamic synergism; alternative drug } \\
\text { chlorothiazide was used in place of furosemide }\end{array}$ \\
\hline Compliance & Non-compliance & $12(3.7)$ & $\begin{array}{l}\text { Anti-D immunoglobulin is available in the hospital, but the patient } \\
\text { couldn't afford and was not injected }\end{array}$ \\
\hline \multirow[t]{2}{*}{ Other categories } & $\begin{array}{l}\text { Need for an additional } \\
\text { laboratory test }\end{array}$ & $41(12.7)$ & $\begin{array}{l}\text { Patient haematocrit value is not registered to recommend or not iron } \\
\text { supplementation or treatment }\end{array}$ \\
\hline & Incomplete drug order & $3(0.9)$ & $\begin{array}{l}\text { Patient is prescribed with methyldopa } 250 \text { mg (mild pre-eclampsia), } \\
\text { but duration was not indicated }\end{array}$ \\
\hline Total MRPs & & $394(100.0)$ & \\
\hline
\end{tabular}

Abbreviations: BID Bis in die (twice daily); IV Intravenous; mg, milligram; MRP medication-related problem; MRSA Methicillin-resistant Staphylococcus aureus; SCAP Severe Community-Acquired Pneumonia; PRN, as needed; PO Per os (by mouth or orally)

a Percentage is calculated taking those with $\geq$ MRP as denominator, $N=323$. Percentage may exceed $100 \%$ due to more than one MRP per patient 


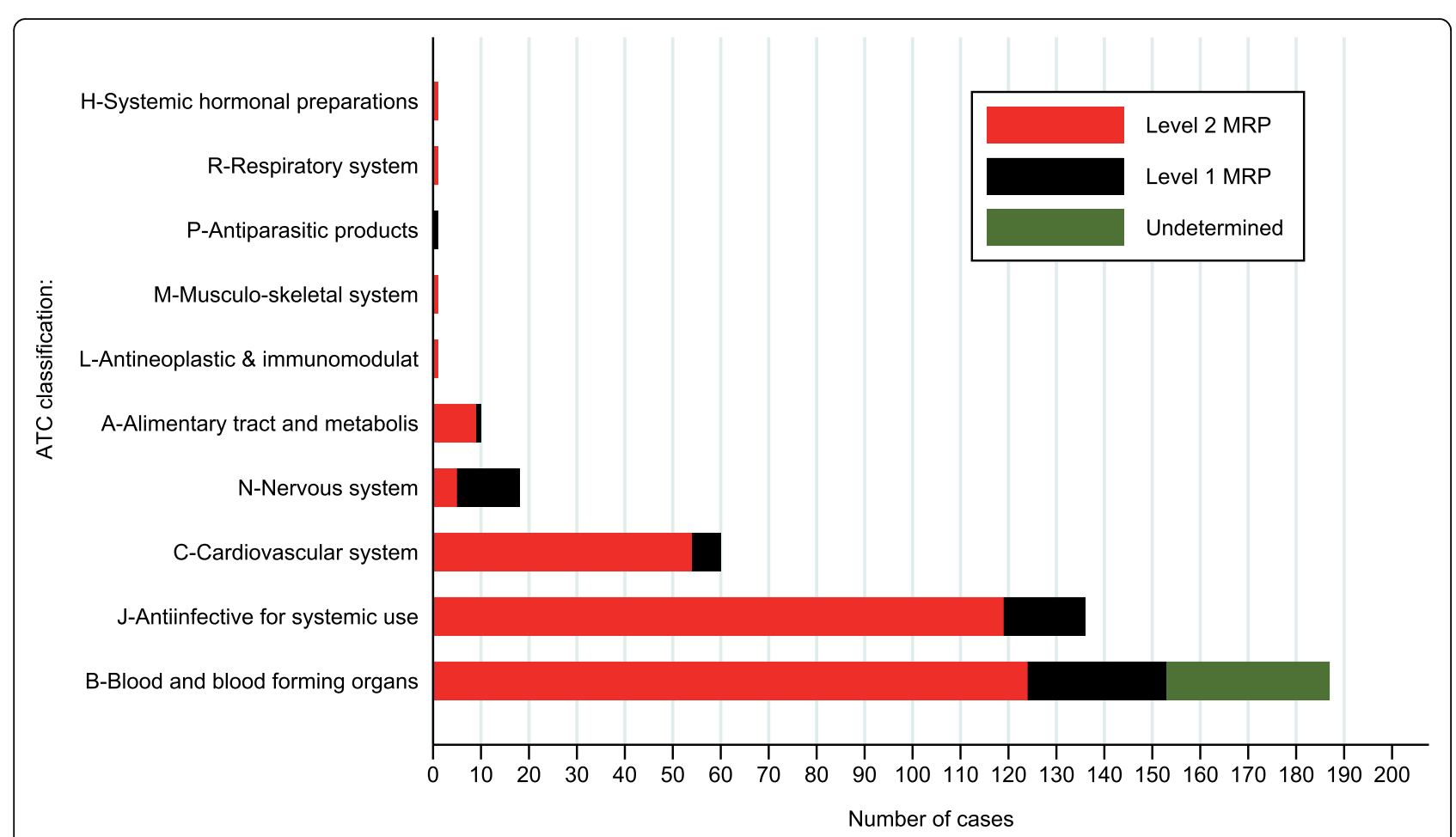

Fig. 1 Overview of the medication groups (by ATC classification system) most commonly involved in MRPs according to severity of the MRP

Another possible reason for the difference in results could be differences in health care systems, the study populations, and disease distribution.

In agreement with the study conducted in Norway [4], 6 out of 10 MRPs in our study concerned the need for additional medication therapy because of untreated illness, mostly in relation to anaemia. In contrast, the study from Australia found that "incomplete medications charted on admission" (28\%) and "incomplete drug order" (15\%) were the two most prevalent MRP categories [3]. The difference may be due to the fact that the Australian study group was able to perform a formal medication reconciliation, which we were not able to do.

Medications acting on the blood and blood-forming organs, anti-infectives for systemic use, cardiovascular drugs, and drugs acting on the nervous system were the medications most commonly involved in MRPs in our study. These findings are relatively similar to prior studies. Antibiotics and iron were the second and third most frequently associated medications in MRPs in the previous Norwegian study [4]. In Australia, most MRPs were related to the alimentary tract and metabolism, and drugs for the nervous system (largely analgesics and antidepressants) [3]. These medication groups may need to be specific focus of the global perspective of MRPs.

Identifying patients with an increased risk of MRPs can be a useful guide for prioritizing tasks in the ward. Our study implies that the focus should be on women with a chronic disease and on women with prior medication use, which is in line with the findings of previous studies [3, 4]. Interestingly, parity was a risk factor for both iron-related MRPs and non-iron-related MRPs, whereas chronic disease and prior medication use were only risk factors for noniron-related MRPs. This may be due to patients with chronic diseases being more likely to use multiple medications, increasing the risk of drug interactions and non-adherence, which in turn increases the risk of MRPs.

Almost half of the moderate to severe (level 2) MRPs (47.8\%) were due to lack of iron supplementation. Another 34 cases had unknown haematocrit due to forgetfulness/lack of time to order standard blood tests or to record patient haematocrit values. Maternal iron deficiency anaemia during pregnancy is associated with multiple adverse outcomes for both mother and infant, including an increased risk of low birth weight, maternal mortality, perinatal mortality, and preterm birth, and is a recognized global problem [28]. This highlights the importance of ensuring an appropriate iron status during pregnancy and after delivery. Giving advice and ensuring that women in need of iron supplements receive it may be the most easily achieved measure to reduce MPRs in maternity care. 


\section{Strengths and limitations}

This study has several strengths, including both its size and the detail of data collected. The use of a standardized system for identifying MRPs, a standardized and systematic chart review at several points during hospitalization, and involvement of a panel of experts in MRP identification are additional important strengths of the present study. Moreover, health care personnel from Ethiopia with knowledge of the healthcare system, local language, culture, and previous research or practice experience performed the data collection.

A major limitation of this study is that our results depended on the accuracy of the chart recording by health professionals. Lack of recording/documentation occurs frequently due to lack of time, and the MRP identification panel agreed to consider it as a documentation problem rather than an MRP for the current study. This will result in an underestimation of the actual number of MRPs. Moreover, JUMC is a referral hospital with a larger proportion of women with pregnancy complications, and possibly with a higher need for medications. As such, the findings will probably not be representative of primary or secondary care services. Finally, as it was difficult to get the patient's full preadmission medication history, no formal medication reconciliation was performed and this could have underestimated the true prevalence of MRPs.

\section{Conclusions}

This study confirms that MRPs are common among women in maternity and gynaecological wards. The most common MRPs were need for additional iron drug therapy, need for additional laboratory test, unnecessary drug therapy, and too low dose of medication. The most important factor associated with MRPs in pregnancy were parity, prior medication use, and chronic illness. Increased adherence to iron supplementation guidelines in inpatient maternity care is urgently needed. Future research should address whether interdisciplinary teams and clinical pharmacy services in the maternity and gynaecology ward can reduce the frequency and consequences of MRPs, and whether this leads to improvements in patient health outcomes.

\section{Supplementary Information}

The online version contains supplementary material available at https://doi. org/10.1186/s12884-020-03433-6.

Additional file 1:. Identification, assessment, classification, and documentation of MRPs and recommendations by the panel of experts

Additional file 2:. Characteristics of the study population according to ward type at JUMC, Ethiopia, from February to June 2017

Additional file 3:. Medications used among hospitalized pregnant women at JUMC, Ethiopia, from February to June 2017
Additional file 4:. Discharge medications prescribed to hospitalized pregnant women at JUMC, Ethiopia, from February to June 2017

Additional file 5:. Types and examples of MRPs identified among hospitalized pregnant women at JUMC, Ethiopia, from February to June 2017

Additional file 6:. Medications involved in MRPs among hospitalized pregnant women at JUMC, Ethiopia, from February to June 2017

\section{Abbreviations}

ANC: Antenatal care; ATC: Anatomical therapeutic chemical classification system; Cl: Confidence interval; HEG: Hyperemesis gravidarum; JUMC: Jimma University medical centre; MMR: Maternal mortality ratio; MRP: Medicationrelated problem; OR: Odds ratio; WHO: World health Organization

\section{Acknowledgements}

The authors thank all of the women who consented to participate in this study. We are also very grateful to the pharmacists, nurses, and physicians who skilfully collected the data and were involved in the expert decisions in MRP identification. We are much indebted to Mr. Bodena Bayissa (clinical pharmacist and lecturer) for his pivotal role in the MRP panel of experts. A special acknowledgement also goes to the Norwegian PhD School in Pharmaceutical Sciences for travel grant assistance for the data collection. Many thanks also to Dr. Ibrahimu Mdala for assisting with the data analysis. Sincere appreciation is extended to the management and staff of Jimma University Medical Centre for making it possible to perform this study.

\section{Authors' contributions}

All authors critically reviewed, contributed important intellectual content to, read, and gave their approval of the final manuscript. SMA and HN conceived the idea for the study and its design. SMA collected, analysed, and interpreted the data and wrote the draft manuscript. YAA and JS did on site supervision of data collection. SMA and $\mathrm{HN}$ revised and finalized the paper.

\section{Funding}

SMA was a PhD student at the University of Oslo during the study. He received a travel grant from Norwegian PhD School in Pharmaceutical Sciences for the data collection. He was also a recipient of a scholarship from the Norwegian Loan Fund (Lånekassen). These institutes were not involved in the design of the study or the collection, analysis, and interpretation of data, or in writing the paper.

\section{Availability of data and materials}

The datasets used during the current study are available from the corresponding author upon reasonable request.

\section{Ethics approval and consent to participate}

The Institutional Review Board, Institute of Health, Jimma University, Ethiopia (date of approval: 17 January 2017; reference number IHRPGC 7206/07), and the Regional Committees for Medical and Health Research Ethics in Norway (date of approval: 17 December 2015; reference number 2015/2135, REK SørØst B) approved the study. All information collected from participants during the study was kept confidential. All individual participants included in the study provided written informed consent.

\section{Consent for publication}

Not Applicable.

\section{Competing interests}

The authors confirm that they have no competing interest.

\section{Author details}

${ }^{1}$ Department of Community Medicine and Global Health, Institute of Health and Society, Faculty of Medicine, University of Oslo, Oslo, Norway. ${ }^{2}$ Division of Social and Administrative Pharmacy, School of Pharmacy, Faculty of Health Sciences, Jimma Institute of Health, Jimma University, Jimma, Ethiopia. ${ }^{3}$ Department of Obstetrics and Gynaecology, Faculty of Medical Sciences, Jimma Institute of Health, Jimma University, Jimma, Ethiopia.

${ }^{4}$ Pharmacoepidemiology and Drug Safety Research Group, Department of Pharmacy, Faculty of Mathematics and Natural Sciences, University of Oslo, 
Oslo, Norway. ${ }^{5}$ Department of Child Health and Development, Norwegian Institute of Public Health, Oslo, Norway.

Received: 13 May 2020 Accepted: 17 November 2020

Published online: 26 November 2020

\section{References}

1. Cipolle RJ, Strand LM, Morley PC. Pharmaceutical care practice: the patientcentered approach to medication management services. 3rd ed. New York: McGraw-Hill; 2012.

2. PCNEA 2003-2019. PCNE Classification for Drug-Related Problems V9.00. 2019. https://www.pcne.org/upload/files/334_PCNE_classification_V9-0.pdf. Accessed 12 Sep 2019.

3. Thompson R, Whennan L, Liang J, Alderman C, Grzeskowiak LE. Investigating the frequency and nature of medication-related problems in the Women's health unit of an Australian tertiary teaching hospital. Ann Pharmacother. 2015;49(7):770-6.

4. Smedberg J, Brathen M, Waka MS, Jacobsen AF, Gjerdalen G, Nordeng H. Medication use and drug-related problems among women at maternity wards-a cross-sectional study from two Norwegian hospitals. Eur J Clin Pharmacol. 2016;72(7):849-57.

5. Deepishka P, Gali SD, Arcot M, Durga Prasad TS. Assessment of drug related problems and clinical pharmacist interventions in paediatric department of a tertiary care teaching hospital. Int J Basic Clin Pharmacol. 2018;7(10):19349.

6. Ayalew MB, Megersa TN, Mengistu YT. Drug-related problems in medical wards of Tikur Anbessa specialized hospital, Ethiopia. J Res Pharm Pract. 2015;4(4):216-21.

7. Fernandez-Llamazares CM, Calleja-Hernandez MA, Manrique-Rodriguez S, Perez-Sanz C, Duran-Garcia E, Sanjurjo-Saez M. Prescribing errors intercepted by clinical pharmacists in paediatrics and obstetrics in a tertiary hospital in Spain. Eur J Clin Pharmacol. 2012;68(9):1339-45.

8. Kandil M, Sayyed T, Emarh M, Ellakwa H, Masood A. Medication errors in the obstetrics emergency ward in a low resource setting. J Matern Fetal Neonatal Med. 2012;25(8):1379-82.

9. Little JA, Velazquez MB, Rayburn WF. Reported medication errors in obstetric inpatients in 1 hospital. J Reprod Med. 2003:48(10):818-20.

10. Kebede B, Gedif T, Getachew A. Assessment of drug use among pregnant women in Addis Ababa, Ethiopia. Pharmacoepidemiol Drug Saf. 2009;18(6): 462-8.

11. Beyene KGM, Beza SW. Self-medication practice and associated factors among pregnant women in Addis Ababa, Ethiopia. Trop Med Health. 2018; 46:10.

12. Molla F, Assen A, Abrha S, Masresha B, Gashaw A, Wondimu A, Belete Y, Melkam W. Prescription drug use during pregnancy in southern Tigray region, North Ethiopia. BMC Pregnancy Childbirth. 2017:17:170

13. Mohammed MA, Ahmed JH, Bushra AW, Aljadhey HS. Medications use among pregnant women in Ethiopia: a cross sectional study. J Appl Pharm Sci. 2013;3(4):116-23.

14. Birarra MK, Heye TB, Shibeshi W. Assessment of drug-related problems in pediatric ward of Zewditu memorial referral hospital, Addis Ababa, Ethiopia. Int J Clin Pharm. 2017:39(5):1039-46.

15. Yimama M, Jarso H, Desse TA. Determinants of drug-related problems among ambulatory type 2 diabetes patients with hypertension comorbidity in Southwest Ethiopia: a prospective cross sectional study. BMC Res Notes. 2018;11:679.

16. Tigabu BM, Daba D, Habte B. Drug-related problems among medical ward patients in Jimma university specialized hospital, Southwest Ethiopia. J Res Pharm Pract. 2014;3(1):1-5

17. Niriayo YL, Kumela K, Kassa TD, Angamo MT. Drug therapy problems and contributing factors in the management of heart failure patients in Jimma University specialized hospital, Southwest Ethiopia. PLoS One. 2018;13(10): e0206120.

18. Jimma University. 2018. Jimma University Specialized hospital. http://www ju.edu.et/jimma-university-specialized-hospital-jush. Accessed 10 Nov 2018

19. Segni H, Ayana D, Jarso H. Prevalence of hyperemesis Gravidarum and associated factors among pregnant women at Jimma University medical center, south West Ethiopia: a cross-sectional study. EC Gynaecol. 2016;3(5): 376-87.

20. Wolde $Z$, Segni $H$, Woldie M. Hypertensive disorders of pregnancy in Jimma university specialized hospital. Ethiop J Health Sci. 2011;21(3):147-54.
21. Woldeyes WS, Asefa D, Muleta G. Incidence and determinants of severe maternal outcome in Jimma University teaching hospital, south-West Ethiopia: a prospective cross-sectional study. BMC Pregnancy Childbirth. 2018;18:255.

22. Gabbe S, Niebyl J, Galan H, Jauniaux E, Landon M, Simpson J, Driscoll D. Obstetrics: Normal and problem pregnancies. 6th ed. Philadelphia: Saunders; 2012.

23. Food, Medicine and Healthcare Administration and Control Authority of Ethiopia. Standard Treatment Guidelines for General Hospital. 3rd ed. Addis Ababa: Food, Medicine and Health Care Administration and Control Authority (FMHACA); 2014.

24. Ward KE, O'Brien BM. Pregnancy and lactation: therapeutic considerations. In: Dipiro JT, Talbert RL, Yee GC, Matzke GR, Wells BG, Posey LM, editors. Pharmacotherapy: a pathophysiologic approach. 9th ed. New York: McGrawHill; 2015. p. 257-300.

25. World Health Organization. Pregnancy, Childbirth, Postpartum and Newborn Care: A Guide for Essential Practice. 2015 https://www.who.int/maternal_ child_adolescent/documents/imca-essential-practice-guide/en/. Accesse- 21 Oct 2019.

26. Cornish PL, Knowles SR, Marchesano R, Tam V, Shadowitz S, Juurlink DN, Etchells EE. Unintended medication discrepancies at the time of hospital admission. Arch Intern Med. 2005;165(4):424-9.

27. World Health Organization: WHO Collaborating Centre for Drug Statistics Methodology, Guidelines for ATC classification and DDD assignment 2016, Oslo, Norway; 2017

28. World Health Organization. Iron Deficiency Anaemia; Assessment, Prevention and Control: A guide for programme managers. 2001. https:// www.who.int/nutrition/publications/en/ida_assessment_prevention_control. pdf. Accessed 5 Dec 2019

\section{Publisher's Note}

Springer Nature remains neutral with regard to jurisdictional claims in published maps and institutional affiliations.

\section{Ready to submit your research? Choose BMC and benefit from: \\ - fast, convenient online submission \\ - thorough peer review by experienced researchers in your field \\ - rapid publication on acceptance \\ - support for research data, including large and complex data types \\ - gold Open Access which fosters wider collaboration and increased citations \\ - maximum visibility for your research: over 100M website views per year}

At BMC, research is always in progress.

Learn more biomedcentral.com/submissions 